\title{
The gut epithelium from feeding to fasting in the predatory soil mite Pergamasus longicornis (Mesostigmata: Parasitidae): one tissue, two roles
}

\author{
Clive E. Bowman ${ }^{1}$ (D)
}

Received: 6 August 2018 / Accepted: 27 February 2019 / Published online: 20 March 2019

(c) The Author(s) 2019

\begin{abstract}
A review of acarine gut physiology based on published narratives dispersed over the historical international literature is given. Then, in an experimental study of the free-living predatory soil mite Pergamasus longicornis (Berlese), quantitative micro-anatomical changes in the gut epithelium are critically assessed from a temporal series of histological sections during and after feeding on larval dipteran prey. An argued functional synthesis based upon comparative kinetics is offered for verification in other mesostigmatids. Midand hind-gut epithelia cell types interconvert in a rational way dependent upon the physical consequences of ingestion, absorption and egestion. The fasted transitional pseudostratified epithelium rapidly becomes first squamous on prey ingestion (by stretching), then columnar during digestion before confirmed partial disintegration (gut 'lumenation') during egestion back to a pseudo-stratified state. Exponential processes within the mid- and endodermic hind-gut exhibit 'stiff' dynamics. Cells expand rapidly $\left(t_{1 / 2}=22.9-49.5 \mathrm{~min}\right)$ and vacuolate quickly $\left(t_{1 / 2}=1.1 \mathrm{~h}\right)$. Cells shrink very slowly $\left(t_{1 / 2}=4.9\right.$ days $)$ and devacuolate gently $\left(t_{1 / 2}=1.0-1.7\right.$ days $)$. Egestive cellular degeneration has an initial $t_{1 / 2}=7.7 \mathrm{~h}$. Digestion appears to be triggered by maximum gut expansion-estimated at 10 min post start of feeding. Synchrony with changes in gut lumen contents suggests common changes in physiological function over time for the cells as a whole tightly-coupled epithelium. Distinct in architecture as a tissue over time the various constituent cell types appear functionally the same. Functional phases are: early fluid transportation $(0-1 \mathrm{~h})$ and extracellular activity (10-90 $\mathrm{min}$ ); through rising food absorption (10 $\mathrm{min}$ to $>1$ day); to slow intracellular meal processing and degenerative egestive waste material production ( 1 to $>12$ days) much as in ticks. The same epithelium is both absorptive and degenerative in role. The switch in predominant physiology begins $4 \mathrm{~h}$ after the start of feeding. Two separate pulses of clavate cells appear to be a mechanism to facilitate transport by increasing epithelial surface area in contact with the lumen. Free-floating cells may augment early extracellular lumenal digestion. Possible evidence for salivary enzyme alkaline-related extra-corporeal digestion was found. Giant mycetome-like cells were found embedded in the mid-gut wall. Anteriorly, the mid-gut behaves like a temporally expendable food processing tissue and minor long-term resistive store. Posteriorly the mid-gut behaves like a major assimilative/ catabolic tissue and 'last-out' food depot (i.e., a 'hepatopancreas' function) allowing the mite to resist starvation for up to 3.5 weeks after a single meal. A 'conveyor-belt' wave
\end{abstract}

Extended author information available on the last page of the article 
of physiology (i.e., feeding and digestion, then egestion and excretion) sweeps posteriorly but not necessarily pygidially over time. Assimilation efficiency is estimated at $82 \%$. The total feeding cycle time histologically from a single meal allowing for the bulk of intracellular digestion and egestive release is not $52.5 \mathrm{~h}$ but of the order of 6 days ( $\equiv 0.17$ total gut emptyings per day), plus typically a further 3 days for subsequent excretion to occur. Final complete gut system clearance in this cryptozooid may take much longer ( $>15$ days). A common physiology across the anactinotrichid acarines is proposed. A look to the future of this field is included.

Keywords Anactinotrichida $\cdot$ Digestion $\cdot$ Egestion $\cdot$ FIFO $\cdot$ FILO $\cdot$ Histochemistry $\cdot$ Hysteresis · Input-output · Kinetics · LIFO · Light microscopy · LILO · Pulse-chase

\section{Review of the field}

As Yonge (1928) says: "A sound knowledge of the structure and function of the feeding organs is of the first importance in the study of the living animal". This is true for understanding the physiological adaptations of predatory soil arthropods such as the mite Pergamasus longicornis (Berlese) (Chelicerata: Acarina: Mesostigmata: Parasitidae) as much as any other metazoan. Internal anatomy is a key aspect of this. Chelicerates were some of the first arthropods to be investigated by digestive physiologists over 150 years ago (Müller 1828; Blanchard 1855b; Dufour 1856; Ponton 1868; Plateau 1876; Croneberg 1878a; Krunkenberg 1880; Bertkau 1884; Macleod 1884b; Birula 1891; Bernard 1893). Despite world events, this was diligently pursued into the early years of the twentieth century (Pocock 1902; Kobert 1903; Oetcke 1912; Hamburger 1916-1917; Krüger 1934; Millot 1931b, 1942, 1943, 1945a, b; Schlottke 1936; Frank 1938; Pickford 1942). However, despite the painstaking early European anatomical work (Claparède 1868; Haller 1880; Henking 1882; Michael 1883, 1884, 1892, 1894, 1895, 1896b, 1901, 1903; MacLeod 1884a; Nalepa 1884, 1885, 1887; Mégnin 1886; Karpelles 1893; Neri 1896; Berlese 1897, 1899, 1918; Pollock 1898), the fine histological detail of acarine tissues remains poorly known.

Although in the early to mid twentieth century, major zoological summary works in French and German were drawn up (Daiber 1921; Pawlowsky 1927; Thor 1931; Kästner 1940; André 1949; Buddenbrock 1956) building upon Napoleonic and Victorian efforts (Treviranus 1812; Lang 1891; Griffiths 1892), these contained limited material on arachnid micro-anatomy and nutritive physiology in the round. The early detailed descriptions and process narratives (Biedermann 1911) have stood the test of time (Romijn 1946) although they may not be completely correct (Richards and Fry 1978). General zoological (Snodgrass 1952) and specialist histological text books (Andrew 1959; Leake 1975) are scant on very much more detail. Relevant primary literature is scattered over a wide variety of biological journals. As Legendre (1968) said: "L'anatomie interne des Acariens est relativement mal connue."-matters have not changed much in the interim. An up-to-date review and source document is needed. Recently an attempt was made to draw together much of the highly dispersed microscopic anatomical knowledge of chelicerates into an illustrated reference on the functional anatomy of invertebrates (Harrison and Foelix 1999).

This review focuses upon the structure and physiology of the acarine gut, digestion and excretion. It does not cover the: mouthparts/gnathosoma (and feeding mechanism); salivary glands in detail; cuticle/integument and moulting; sense organs (including eyes and the effects of light or photoperiod); dermal glands; muscles; respiratory/tracheal systems 
(including peritremes); neural systems in detail; neuroendocrine systems; or anything solely related to reproduction or cytogenetics. These await a further review.

There is a large corpus of descriptive anatomical work on blood feeding ticks, started by Heller (1858) and Pagenstecher (1861) and pursued by workers such as: Allen (1905), Nordenskiöld (1905, 1906, 1908, 1909, 1911), Williams (1905), Bonnet (1906, 1907, 1908), Christophers (1906), Samson (1909), Blanc (1910), Robinson and Davidson (1913-1914), True (1932), Müller (1939), Stella (1942), Douglas (1943), Hughes (1954b), Schulze (1943), Balashov (1957a, b, 1988), Ohara and Homma (1959), Saito (1897), Roshdy (1961a, 1962, 1963), Chinery (1964), Tatchell (1964), Arthur (1965), Roshdy (1966), Efremova (1967), Aeschlimann and Ryhiner (1970), Sonenshine (1970), Sonenshine and Gregson (1970), Belozerov and Tymopheev (1973), Guirgis (1971), Khalil (1971), Tatchell et al. (1973), Balashov and Raikhel (1974, 1975, 1976a, b, 1977), Obenchain and Oliver (1976, 1978), Maathai (1977), Smit et al. (1977), Roshdy and Marzouk (1984), Agbede and Kemp (1985, 1987) and Agbede (1986) etc, for many years mainly in the US and the then-called USSR. Illustrative material on this can be found in the atlas by Coons and Alberti (1999).

Outside of ticks, historically most micro-anatomical effort has been made on astigmatid and trombidiid mites. Illustrative material on them can be found in the atlas by Alberti and Coons (1999). Notostigmatids (With 1904); holothyrids (Thon 1905b, c, d; Walter and Proctor 1998) and opilioacarids Vitzthum (1940) are not well known at all.

For specific economically important astigmatids relevant works are: Boczek et al. (1969) for Acarus farris, Hughes (1950a) and Sobotnik et al. (2008a) for Acarus siro, Akimov and Starovir (1980) for Acotyledon absoloni, Vijayambika and John (1977) for Aleuroglyphus ovatus; Wu et al. (2009) for Blomia tropicalis, Woodring and Carter (1974) for Caloglyphus boharti, Rohde and Oemick (1967) and Kuo and Nesbitt (1970, 1971) for Caloglyphus mycophagus, Oboussier (1939) for Caloglyphus spinitarsus, Prasse (1967, 1968a, b) for Caloglyphus spp., Oboussier (1939) for Carpoglyphus lactis, Nevin (1935) for Cnemidocoptes mutans, Brody and Wharton (1970, Brody 1971, Brody et al. 1972, 1976, Tongu et al. 1986, Zhang et al. 2008 and Wang et al. 2013) for Dermatophagoides farinae, Tongu et al. (1986) for Dermatophagoides pteronyssinus, Lönnfors (1930) and Dubinin (1951) for feather mites; Oboussier (1939) for Glycyphagus cadaverum, Bekker (1940) for Glycyphagus destructor, Oboussier (1939) and Hughes and Hughes (1939) for Glycyphagus domesticus, Baker (1975) for Histiogaster carpio, Perron (1954) for Histiostoma laboratorium, Behura (1956) for Histiostoma polypori, Bücking (2002) for Hyadesia fusca, Langenscheidt (1958) for Knemidocoptes mutans, Vijayambika and John (1974, 1975a, b, c, d, 1976a, b, c) for Lardoglyphus konoi, Hughes (1954a) for Listrophorus leukarti; Gudden (1861), Heilesen (1946) and Desch et al. (1991) for Sarcoptes scabiei, Bekker (1959) and Akimov (1973, 1975) for Rhizoglyphus echinopus, Bekker (1940) for Tyroglyphus farinae; and Oboussier (1939) for Tyrophagus dimidiatus.

For the Trombidiformes particularly relevant studies by publication year are: Pagenstecher (1860), Croneberg (1878b, 1879), Kramer (1885), Schaub (1888), Michael (1896a), Nordenskiöld (1898, 1900), Brucker (1900), Thor (1902, 1904), Thon (1903, 1905a), Reuter (1909), Newstead and Duvall (1918), Brown (1922, 1952), Steding (1924), Thomae (1925), André (1927), Hassan (1928), Lundblad (1930), Hafiz (1935), Schmidt (1935), Bader (1938, 1954, 1969), Grandjean (1939), Volkonsky (1940), Lombardini (1942), Blauvelt (1945), Turk and Phillips (1946), Reiff (1949), Jones (1950), Gasser (1951), Wharton and Fuller (1952), Stout (1953), Klumpp (1954), Obata (1954), Mitchell (1955, 1964, 1970), Schnieder-Berkenbosch (1955), Ehara (1960), Ashton (1961), Moss (1962), Anwarullah (1963), Wright and Newell (1964), Easwari Arama (1967), Wiesmann (1968), 
Jalil (1969), Baker (1970, 1971, 1973, 1977), Silvere (1971), Alberti (1972, 1973), Whitmoyer et al. (1972), Witte (1972), Ehrnsberger (1973), Sixl (1973), Summers et al. (1973), Nuzzaci (1976), Akimov and Barabanova (1977), Vistorin-Theis (1977b), Schramlová (1978a, b), Nuzzaci (1979), Paran (1979), Berezantsev (1980), Crooker (1980), Mothes and Seitz (1980), Vistorin (1980), Mothes and Seitz (1981b), Shatrov (1983), Akimov and Gorgol' (1984, 1987), Mothes-Wagner (1985), Barabanova (1993), Filimonova (2001, 2008a, b, 2013), Shatrov (2010), Alberti et al. (2014), Vasquez (2017) and Bensoussan et al. (2018).

Oribatids have been poorly studied (Tarras-Wahlberg 1960; Woodring and Cook 1962; Hoebel-Mävers 1967; Tarman 1968; Bernini 1971; Haarløv and Bresciani 1972; Woodring 1973; Dinsdale 1974, 1975; Smrž 1989; Šustr and Hubert 1999; Bücking 2002; Alberti et al. 2003). Given their predominance in soils and plethora of species feeding on different material, much more work is needed on cryptostigmatid physiology.

The general internal anatomy of anactinotrichid gamasids (Acarina: Mesostigmata) has been qualitatively known for over a 100 years (Mégnin 1876; Winkler 1888; Michael 1889). However, apart from some pest species (Hughes 1952; Jakeman and Strandtmann 1960; Jakeman 1961; Woodring and Galbraith 1976; Gorgol 1991; Lagutenko 1962; Pritchard et al. 2015) and one free-living macrochelid (Butler 1964; Coons 1978), the mesostigmatid gut and its physiology are not so well researched as ticks. There is some detail on gamasids in Schulze (1943) and material on the internal anatomy Neonyssus melloi by Crossley in Strandtmann and Wharton (1958). Latterly Ukrainian acarologists (led by Akimov in 1970s-1980s in the then-called USSR) have made multiple descriptive investigations of the gut in free-living predatory phytoseiids and amblyseiids (Akimov and Starovir 1983; Starovir 1985; see also Bregetova 1979). There is also an unpublished thesis by Di Palma (1996) dealing with Typhlodromus rhenanoides and Typhlodromus exhilaratus (Mesostigmata: Phytoseiidae) available. Treat (1975) illustrates the internal anatomy of Dicrocheles phalaenodectes (Laelapidae), and Obenchain and Oliver (1973) talks about the fat body and associated tissues in Dermanyssus spp. Balashov (1964) records erythrocyte destruction in the gut of $D$. gallinae.

The anterior or foregut of mites and ticks (comprised of the mouth, pharynx and oesophagus) is ectodermic (Legendre 1967, 1968) and chitin lined (as is most of the respiratory system-Witalinski 1980). The acarine mid-gut however is endodermic and cellular in structure, comprising a ventriculus and numerous blind-ending ramified caeca (diverticula) sheathed by muscle fibres (Vitzthum 1940; Ainscough 1960; Young 1968a; Kaestner 1969a; Caperucci et al. 2010) which drives peristalsis (Stanley 1931). Starovir (1979a) claims that in Amblyseius herbarius the diverticula are adapted for predation being able to absorb food in quantity. Sexual dimorphism in caeca is known in some species (Strandtmann and Wharton 1958). In the mesostigmatid P. longicornis the central bulk of the mid-gut to which the ventriculus and caeca join is labelled as the 'mesenteron' (Fig. 1 in Bowman 2014). It is more than just the meeting point of caeca as in Fuscuropoda spp., being distinct in pergamasids (as in Leiodynichus spp.). From it, that part of the hind-gut being also endodermic in origin is cellular and tubular in gamasids (i.e., it is continuous with the mid-gut without a clear separation as in Leiodynichus and without a muscular sphincter-just as in ixodids, Ainscough 1960). It leads to a junction with the excretory Malpighian tubules and the ectodermic chitin-lined membraneous rectal vesicle. This contrasts with the blind gut of some actinotrichids where there is no posterior connection [e.g. "der Ringförmige Mitteldarm"-Bader 1954; and in hydrachnids_Pollock (1898)]. The rectal vesicle in gamasid mites is a storage organ from which faeces are voided via muscular action through the anus (Heethoff and Norton 2009). There is no clear digestively active 
thoracentron nor extensive chylenteron as in spiders (Millot 1926, 1931a; Hickman 1942) nor multiple diverticular types as in solifugids (Klann 2009). The question of a 'liver' in chelicerates has been around for a century- Jordan (1912). However, no distinct cephalothoracic liver-like organ as in scorpions (nor indeed a pancreas) has been found in mesostigmatid mites (Kaestner 1969b). Digestive products are distributed by open circulation in a diffuse haemocoel (Treat 1975).

Recent histologists and cytologists have mainly focused their efforts on describing the fine detail of acarine reproductive systems abutting the gut (Warren 1940, 1941, 1947; Young 1968b; Petrova 1970; Alberti and Storch 1973; Witte and Storch 1974; Alberti 1977, 1980a, b; Mothes and Seitz 1981a; Akimov and Yastrebtsov 1984, 1985; Alberti and Hänel 1986; Akimov et al. 1988; Walzl 1992; Alberti et al. 1999; Nuzzaci et al. 2001; Lekimme et al. 2005; or for a pergamasid-Witalinski 1975). Acarine coxal glands have been intensively examined (Groepler 1969; Alberti et al. 1996). Various workers have researched salivary glands (Coons and Roshdy 1973) or neural organs (Eichenberger 1970; Coons and Axtell 1971b). However, unlike in other arachnids (Alberti and Storch 1983), gut histologies have been infrequently researched with many histologists appearing to have been content with just a subjective assessment of the course of histodynamics. The presence of cilia in the acarine gut has been claimed (Rohde and Oemick 1967). The presence of microvilli in the acarine gut has also been claimed (Tatchell 1964; Brody et al. 1972; Balashov and Raikhel 1975; Nuzzaci 1976; Chaika 1977; Coons 1978; Akimov and Starovir 1980; Agbede 1986) or at least a brush border to the epithelium (Dinsdale 1974). However, critical quantitative discussion of cellular successions is lacking despite cell growth, vacuolation and tissue disintegration being commonly described gut epithelial modalities.

Reichenow (1918) found that in the dermanyssid mite, Liponyssus saurarum, the large clavate cells of the gut are amoeboid and ingest corpuscles from the blood of the host, digesting them intracellularly. After becoming first gorged with food vacuoles and elongated standing clear on a peduncle, then filled with intracellular waste products (i.e., dark granules, spherical masses and pigment drops), these cells become detached and fall into the lumen of the gut where they disintegrate. They arise from small cubical basal cells in the starved mite. Pavlovsky and Zarin (1926) posed the prevailing view of two chelicerate gut epithelial cell types ('digestive' versus 'secretory') derived from an undifferentiated third ('reserve') form - a scheme followed by Hughes (1952) and Belozerov (1957). The latter author describes a single layer of intestinal epithelial cells elongated in the direction of the lumen resting on a thin basement membrane which is in turn enclosed by a circular musculature of smooth muscle fibres. The epithelial cells are haphazardly arranged to give the impression of a stratified epithelium. During absorption of liquid food by Poecilochirus necrophori (Mesostigmata: Parasitidae), these cells greatly enlarge and fill with vacuoles of different sizes and staining properties. As the parasitid's cells digest the food vacuoles, these cells become gradually filled more and more with drops of excretion until they fill out the lumen of the intestine. Parts of the cells filled with excretion are then pinched off and pass through the hind-gut into the cloaca (= rectal vesicle).

However, as Hoogstraal (1983) points out even for the well-studied ticks the narrative and details of such digestion can be contentious - see for example discussions in Chinery (1964), in El Shoura (1988) and the results of Kanwar and Malik (1966). At first, Balashov and Raikhel (1978) describe three types of midgut cell (reserve, digestive and secretory), but then go on to point out that the reserve and secretory cells dominate one phase of digestion, while the digestive cells which assimilate by both phagocytosis of solids and pinocytosis of liquids another-yet themselves having a "labile" relationship with the secretory cells. Is this then one cell (reserve) with two outcomes (secretory or digestive)? Or, is this one type of cell 
(reserve) with an interchangeably secretory/digestive outcome? Are any of them fundamentally different at all? Various authors using light microscopy describe the sizes, shapes and growth of all sorts of: digestive cells, secretory cells, absorbent cells, ferment cells, B-cells, D1 cells, D2 cells, F-cells, R-cells, basal cells, stem cells, reserve cells, regenerative cells, non-differentiated cells, columnar cells, cylindrical cells, pear-shaped cells, cubic cells, prismatic cells, pyramidal cells, 'spent' cells, empty 'digest' cells etc in diverse acarines claiming unique functions for them (Starovir 1973, 1979a, b, c, 1981a, b, 1982; Akimov and Starovir 1974, 1976, 1977, 1983; Starovir and Barabanova 1981; Akimov and Schlur 1972); or claiming multiple roles for them (Filimonova 2009). What are all these diverse cells?

Much of this work has been on economically important phytoseiids and amblyseiids not just ticks. Confusingly, different historical authors use the same labels for cells which are clearly of different physiological type based upon their annotated illustrations. Recent authors rely upon TEM classification of acarine gut cells based upon their ultrastructure into either: stem/replacement cells and a single type of digestive cells (Tarnowski and Coons 1989), that can contain apparent secretory vacuoles (Balashov and Raikhel 1977); or alternatively a scheme of Type I (secretory) and Type II (digestive) cells (Hamilton et al. 2003). But that does not really help. Many other researchers talk of desquamation, epidermal cell release etc (Arthur 1965). Other authors in explicitly describing acarine cells types use the staining colour of vacuoles to decide if the cells are digestive ('ferment') or secretory. However, none of these descriptions and narratives appear to quantitatively score the prevalence of each cell type. Given the vagaries of histological staining, colour change is poor evidence to ascribe unequivocally the role of any cell. Indeed Balashov and Raikhel (1974) maintain that it is impossible to divide cells as digestive and secretory by their morphological characters in ticks (that are starved). Clarity for mites is needed. Are there truly multiple cell types or not? Is it just a question of nutritive context?

Cell inclusions are often described-but not explicitly enumerated-despite a generally accepted view that much, if not all, of proteolytic digestion in the mid-gut of acarines occurs within the cells (Arthur 1965). Whilst gut $\mathrm{pH}$ has been studied (Popow and Golzowa 1933; Hughes 1950a; Akimov and Barabanova 1976a, b, 1978; Barabanova 1980a; Erban and Hubert 2010b), detailed biochemistry is missing for most acarines-although digestive enzymes (Barabanova 1972, 1975, 1976, 1983, 1984b, 1985), as well as body composition and intermediate metabolic pathways have been investigated especially in tetranychid plant mites (Mehrota 1961, 1962, 1963a, b; Walling et al. 1968; Kötter 1978). Some work in ticks has been carried out-Frayha et al. 1970, 1972; Cherry 1976; Maroun and Kamel 1976-more is needed. Khomyakov (1967) has looked at digestion and nutrition in soil haematophages. Recently an elegant dissection of anatomical and physiological flexibility of Tetranychus urticae has been published-Bensoussan et al. (2018). Astigmatid carbohydrases and their relationship to feeding adaptations have been studied by various workers (Barabanova 1981; Bowman 1984b; Akimov and Oksentyuk 2018) — as have a variety of oribatids been investigated (Šustr and Starý 1998). $\alpha$-Fucosidase and other carbohydrases are known in ticks-Moreti et al. (2013). Fats and sugars have been examined in ticks (Aboul-Nasr and Bassal 1971; Hajjar 1972; Barker and Lehner 1976) as have amino-acids (Stanyukovich 1966; Boctor and Araman 1972; Stepanchenok-Rudnik et al. 1975). What anactinotrichid gut cell inclusions might be physiologically needs clarity. Could they be enzymes or food material or something else?

For mesostigmatids, physiological results from three other recent authors are of note. Intentionally these results cover anactinotrichid acarines, as detailed accounts of organ functioning, gut tissue development, cellular changes, and excretory processes for actinotrichid mites diverge somewhat (see detailed descriptions in Hubert and Šustr 2001; Smrž 2002; Filimonova 2009 for example). 
- Firstly, Neumann (1941) states that the uptake of food and digestion in the parasitid Parasitus kempersi is exactly similar to that in the tick Ixodes as described by Roesler (1934). That is, self-perpetuating undifferentiated cells in the adult parasitid lead onto true gut cells. These are cylindrical or irregularly shaped. True gut cells under starvation yield small club-like secretory cells [especially cardially as in Steding (1924), that then may be lost]. On feeding, true gut cells increase in height, forming fibrillar digestive cells (and probably pseudopodial digestive cells). These develop food balls inside them and intracellular digestion occurs, leading to cell degeneration and the formation of crystals which are voided via the anus.

- Secondly, Vinogradova (1960) looking at the facultative parasites Eulaelaps stabularis, Haemolaelaps glasgowi and Haemogamasus nidi likens their mid-gut histological picture to that described in ixodids by Suvorov (1908), True (1932) and Vitzthum (1940).

- Finally, Lagutenko (1963) describes in detail the histological changes in the gut, Malpighian tubules and ovaries during blood digestion in the haematophagous fowl mite Dermanyssus gallinae. Haemoglobin digestion (O'Hagan 1974) and the accumulation of secretions within gut cells occurs, followed by the shedding of the latter cells or haematin itself into the lumen throughout all of the mid gut. The temporal pattern of guanine in the Malpighian tubules of this species is approximately $180^{\circ}$ out of phase with feeding just like laelaptids (Zamsky 1964) and like in pergamasids (Bowman 2017a).

Is this overall picture typical? Starovir (1973) and Akimov and Starovir (1976) describe a large number of persistent small flat undifferentiated cells (slightly vacuolated and usually overcrowded by other cells) that when phytoseiid mites feed produce smooth digestive cells which can be cast off into the lumen. When the mite is hungry these digestive cells become overall secretory cells with polysaccharide containing small vacuoles like other acarines (Balashov 1972). The apical ends of the secretory cells protrude into the gut lumen. The digestive cells have inflated convex apices and are heavily vacuolated. Rough digestive cells degenerate producing first granules then optically active crystals (refractive grains?-Bowman 2017b; guanine?) ready for loss via the anus. Even when starved some digestive cells remain present. A full series of such cytological changes over time after feeding can also be found copiously described (but not quantitated) for Phytoseiulus persimilis in Akimov and Starovir (1974) where a repeated 10-15 min cycle of activity is postulated. Note that the division of gut cells into secretory or digestive may be arbitrary as it only seems to apply at the beginning and end of the feeding process in phytoseiids. If the gut is full, all the cells are digestive as the food is being utilised very quickly. After 6-9 h the ingested food mass begins to disappear and secretory cells that are ready to receive the next food bolus after $9 \mathrm{~h}$ can be seen. Akimov and Starovir (1977) offers a similar detailed narrative of gut histological responses to feeding in Amblyseius andersoni. Low broad cells appear within 1 min of feeding starting, then large cylindrical distended cells by $20 \mathrm{~min}$ (plus cast off cells). Nutrient granules appear almost immediately. Disintegrating degenerative cells with crystals appear by $30 \mathrm{~min}$. The process lasts through $12 \mathrm{~h}$, culminating in a multi-laminar epithelium (looking like a starved mite) by $24 \mathrm{~h}$.

Considering the above, a common bi-phasic scheme across mesostigmatids might exist if the finer cytological details and histological namings were ignored. Coons (1978) describes the prevailing view of digestion in acarines (both ticks and mites) held through to 1980 . However, little or nothing is known since nor quantitated for the gut epithelium in pergamasids. Moreover, measurements not just narratives are needed. Coons (1978) claims that the large size variation which exists between the same pair of caeca in different macrochelid mites prevents accurate measurements. Even in ticks, only Allen (1905) appears 
to try to quantitatively estimate the extent of gut epithelia. Akimov and co-workers (e.g., Akimov and Starovir 1980) lists sizes of nuclei and sizes of gut cells but inconsistently track these over time in their investigations. Although various tick publications investigate the amount ingested (Snow 1970), or the rate of feeding (Norval and Capitini 1974), or the time course of diverse physiologies, no gut kinetic studies have been traced for mesostigmatids before that of Bowman (2014). A critical quantitative synthesis of mesostigmatid mite gut physiology is needed. Does quantitation back-up histological conjecture?

\section{Rationale for this study}

The common carnivorous mesostigmatid $P$. longicornis (Fig. 1, upper) is a convenient freeliving acarine physiological model due to its large size $(>1 \mathrm{~mm})$, immense polyphagous appetite (Luxton 1966) and ease of handling. The very closely related Gamasus (now Pergamasus) crassipes was the species originally investigated micro-anatomically by Winkler
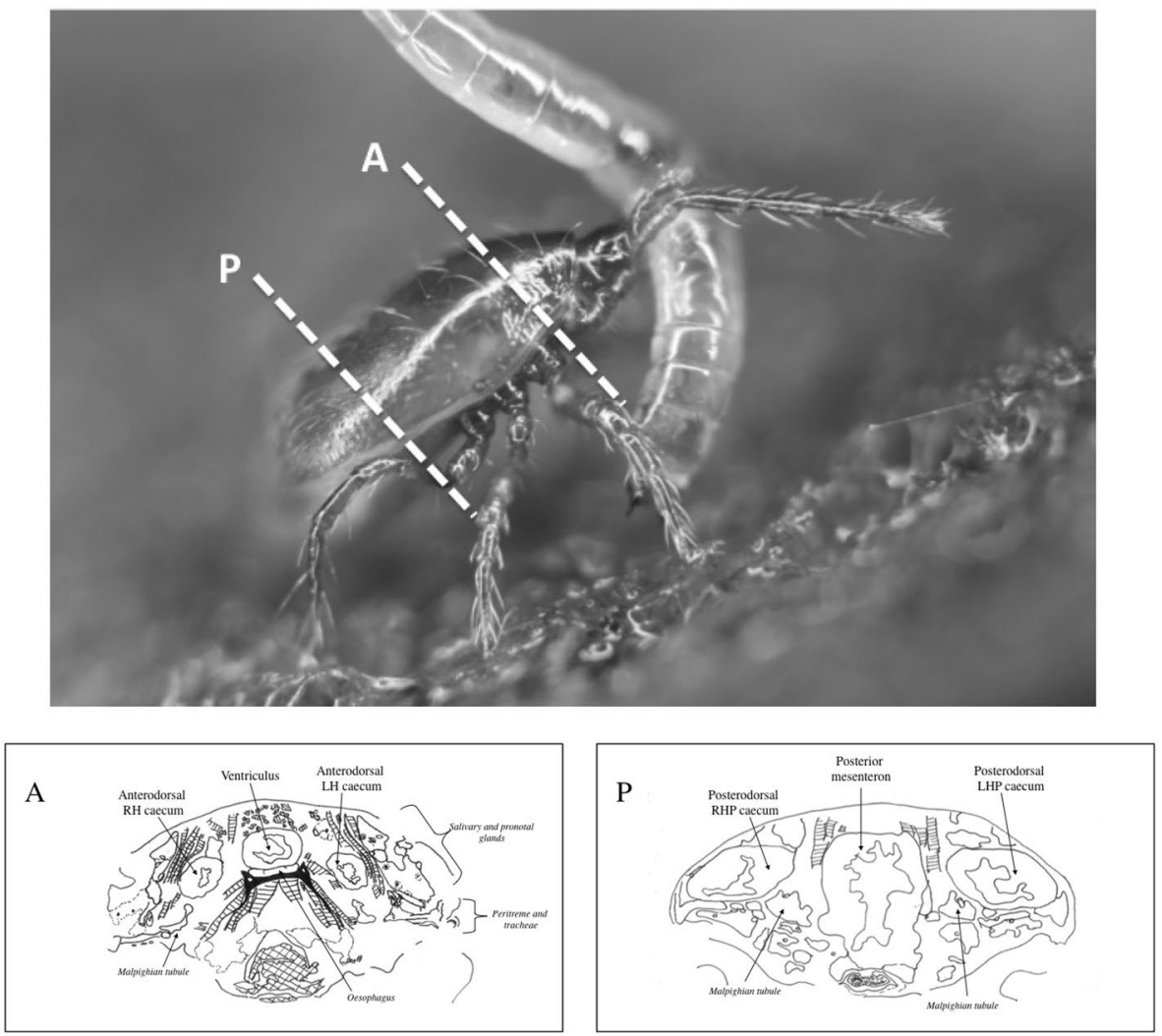

Fig. 1 Pergamasus sp. feeding on chironomid larva. From a colour photograph @Håkon Haraldseide with permission. Dashed lines indicate typical transverse sections generated by this study: $\mathrm{A}=$ anterior; $\mathrm{P}=$ posterior. Many mites were serially sectioned transversely (and longitudinally). Lower: schematic of two transverse sections (TS) showing gut regions ( $P$. longicornis female mite $18 \mathrm{~h}$ after the start of feeding-from Bowman (2014). Left: anterior. Note shrunken mid-gut. Right: posterior. Note expanded mid-gut 
(1888). Adult pergamasids are found in the most varied soils (Kühnelt 1961) being more common in surface litter than in deeper layers (Wallwork 1970); can be kept alive in the laboratory for a long time ( $>24$ weeks at $15^{\circ} \mathrm{C}$-Bhattacharyya 1962); and, are a model for the much smaller economically important phytoseiids. They are top predators in the edaphic acarine world by virtue of their size (Sorensen et al. 1976). Pergamasids feed for anything up to an hour and a half (Bhattacharyya 1962; Bowman 1987a) making detailed physiological observations possible. A longer feeding to fasting cycle is expected than the: 7-8 h digestive pause post-prey capture in Amblyseius largoensis (Sandness and McMurtry 1972); the 1-5 days in Bdellonyssus bacoti (Weitz and Buxton 1953; Sudd 1952); the $\geq 3$ days in typhlodromids (Chant 1958); or, the 2-3 days found in phytoseiids (Barabanova 1980b); again facilitating experimentation.

Being a chelicerate, intracellular digestion is expected as the main gut modality (Snodgrass 1948) rather than also extracellular digestion as in insects-Gooding (1972). However, extracellular digestion occurs to a greater or lesser extent in all Metazoa (with the exception of the Porifera and probably the Brachiopoda-Yonge 1937) and needs evidence looked for in mites. Like spiders (Mommsen 1978a), P. longicornis is not capable of ingesting solid food due to the anatomy of its gnathosoma and foregut. Pre-oral external (aka extra-corporeal) digestion of their prey is therefore expected as it is found in most groups within the Chelicerata (van der Hammen 1977) and specifically in spiders (StradalSchuster 1940-1941) and phytoseiids (Flechtmann and McMurtry 1992). Only one account (Akimov 1977) mentioning membrane (contact) digestion in acarines (Ugolev 1965) has been traced, although Grandjean and Aeschlimann (1973) recount a clear zone of red blood cell rupture in the argasid lumen in the immediate vicinity of gut cells.

The underlying concept of this paper is that gut dynamics over time, point to specific adaptations in the anactinotrichid $P$. longicornis for its trophic environment in the cryptosphere. Measurable gut size changes in accordance with ingestion is expected (as the completely collapsed caeca from the starved state strongly dilate on repletion so that the acarine opisthosoma is distended and turgid Hughes 1959 - see also the situation in sarcoptids Nevin 1935). Mid- and endodermic hind-gut cell types are expected to: wax and wane with high functional activity (like in phytoseiids-Akimov and Starovir 1978; Starovir 1982); then differentiate and interconvert (like in argasids-El Shoura 1988); as the pergamasid gut epithelium changes its form during the process of feeding and digestion on a larval dipteran prey. Opisthosomal colour itself changes in P. longicornis over feeding and excretion (Bowman 2017b), much as the weights of ticks (and optical densities of tick extracts) changes in concert with the colour of their caecal contents (Fig. 3 in Arthur 1965; Fig. 5 in Sutton and Arthur 1962). So the epithelium is thus expected to 'age' physiologically over time through a series of distinct stages related to its nutritional status (Balashov 1961b; Repkina 1976) leading to disintegration as in Amblyseius reductus (Starovir 1982).

Whilst pergamasids eat collembola, nematodes (Blackith 1975) and other cryptozooids, a large 'bolus' of a single larval dipteran prey meal is used so as to maximise the comparability to blood-feeding parasitic ticks (argasids take $5 \mathrm{~min}-2 \mathrm{~h}$ to feed-Arthur 1965, which spans the 56-96 min of the feeding time in P. longicornis observed by Bowman 1987a). The single meal also obviates any confusion with deconvoluting cellular changes induced by a second prey imbibition overlaying any histological changes already induced by a first (or previous) imbibition - as is the risk in studying other mesostigmatids like phytoseiids or macrochelids who eat multiple eggs or tetranychid/nematode prey before satiation. Cell proliferation as in ticks (Gabbay and Warburg 1976) would only be evidentially shown by counting the individual different cell types over time and this is not done 
herein [note that Reichenow (1918) already failed to detect post-embryonic multiplication of cells in the gut wall of L. saurarum].

Hughes (1959) describes small cubical cells as a pavement epithelium in the mite gut interspersed with much larger glandular vacuolated cells which on the much stretching of the gut wall become nipped off into the lumen. These budded off cells being concerned with the accumulation of faecal material (Hughes 1952). In spiders (Fuzita et al. 2016b), vesicular secretions from analogous mid-gut diverticular cells (needed for extra-oral digestion) may be lost in faeces [recall that trypsin protease is found in the faeces of dust mites-Erban et al. (2017), as well as many gut-derived allergen proteins-Vidal-quist et al. (2017)]. Many other studies mention 'clavate' cells protruding into the gut lumen. Different investigators have not been entirely in accord regarding specific cell functions in their descriptions (Snodgrass 1948). So, a focus is made to characterise the pergamasid gut epithelium both numerically and in a 'hypothesis free' manner-i.e., cell function is not assigned a priori. Tracking in detail of the cell appearances (using neutral 'tag' words) is done to inform the inference of what physiological processes may be going on and at what time in the pergamasid. Static anatomical appearance is necessary but insufficient on its own to unequivocally attribute function to a cell. As said by Richard Phillips Feynman (US educator and Nobel Physics prize winning physicist 1918-1988): "You can know the name of a bird in all the languages of the world, but when you're finished, you'll know absolutely nothing whatever about the bird. So let's look at the bird and see what it's doing-that's what counts".

How things work matters-physiological evidence is needed of a cell's adaptations (Yonge 1937, 1954a), not just its micro-anatomical form in deciding its function. Description of epidermal changes in 'pulse-chase' feeding experiments (Thor 1904; Starovir 1982) is useful but quantitation of such narrative changes is also key. Not just tabulations of gut physiological 'age' stages (as in Repkina 1976) but the comparative kinetic measurement of gut dynamics is necessary. Cellular function should be rigorously attributed a posteriori from quantitative assays of the dynamics of cells. Furthermore, any temporal relationships in an organ's cells must also match any coherent explanation of an animal's life-style. Bowman (2014) and Bowman (2017b) already has presented a schema of how the gut overall and the prey food within the pergamasid's gut changes over time and how this can be used for physiological staging (as in ticks Khizhinsky 1968, or in laelaptids Zamsky 1964). Now the matching tissue and cellular changes need to be mapped as well in P. longicornis and a critical quantitative assessment made. Evidence for pergamasid extra-corporeal and extracellular (luminal) enzymatic digestion will also be sought.

In animal cells, 'vacuoles' visible to light microscopy assist in the processes of exocytosis and endocytosis. Exocytosis is the extrusion process of proteins (including enzymes) and lipids from the cell. These materials are first absorbed into secretory granules within the Golgi apparatus before being transported to the cell membrane in a 'vacuole' and secreted into the extracellular environment. In this capacity, vacuoles are simply storage vesicles which allow for the containment, transport and disposal of selected proteins and lipids to the extracellular environment. Exocytosis occurs also of waste products such as residual bodies. Endocytosis is the reverse of exocytosis and can occur in a variety of forms too (Holter 1961). Phagocytosis ('cell eating') is the process by which materials visible under a microscope are engulfed by cells. The material makes contact with the cell membrane, which then invaginates. The invagination is pinched off, leaving the engulfed material in the membrane-enclosed vacuole and the cell membrane intact. Fluid and surface pinocytosis ('cell drinking') are essentially the same vacuole production process but the difference is that substances ingested are in solution and are not visible under the 
microscope (Ganong 2005). Heterophagosomes are formed by the fusion of such phagocytosed material/pinocytotic vesicles with lysosomes carrying intracellular enzymes [like acid phosphatase (Roodyn 1967)] produced by the Golgi/endoplasmic reticulum complex and digestive breakdown takes place. Telolysomes are such lysosomes that have accumulated so much indigestible residue that they are unable to accumulate further material to be digested. Residual bodies containing indigestible material are then either secreted by the cell via exocytosis, or lipase-deficient vesicles become lipofuscin granules that remain in the cytosol indefinitely. Lysosomes are invariably numerous in epithelial cells of organs of absorption, secretion and excretion (Roodyn 1967).

In the closely related to Mesostigmata ixodids, whilst digestion is predominately intracellular (Balashov 1972) within such acidic endosomes/lysosomes (Sonenshine and Roe 1991), it is also extracellular (Grigor'eva 2003, 2004), with the tick gut epithelium markedly changing during feeding (Caperucci et al. 2009). In argasids (Grandjean 1984), another closely related group, the classic intracellular lysosomal mechanism has also been described. In ixodids, food engulfment by typical phagocytosis has been shown-Raikhel (1974b). In spiders after internalization of partially digested food, acidic enzymes become important-Fuzita et al. (2016b). Absorption of prey material or formation of intracellular secretions in P. longicornis should therefore be indicated by both the sizes and form of the gut cells and by the histological appearance of food 'vacuoles' within them. All of these 'vacuoles' will be detectable in light microscopy of $P$. longicornis depending upon the fixation and staining. Phagocytosis of erythrocytes in the gut of the mesostigmatid Liponyssus sp. has already been reported by Reichenow (1921). Endocytosis has been demonstrated in the gut digest cells of the tick Boophilus microplus (Liyou et al. 1996) and in the prostigmatid Myobia murismusculi (Filimonova 2001). In Hyalomma asiaticum, Raikhel (1974b) and Balashov and Raikhel (1976a) have shown both endocytic phagocytosis of blood cells and pinocytosis of haemoglobin by folds and tubules in the digestive cell surface (Balashov and Raikhel 1974). Such endosomal phagocytosis and pinocytosis are both undertaken in association with primary lysosomes (Dean 1977) which complete the breakdown or postprocessing of the material which has been engulfed (Reggiori 2006) - as they merge and form secondary lysosomes. In this heterophagy (Sonenshine and Roe 1991), lysosomes contain proteinases as mature enzymes (Ishidoh and Kominami 2002) for prey tissue lysis. These may be non-selective against long half-life proteins (Dean 1979) and thus useful to a polyphagous predator. Such lysosomes (histologically locatable as stainable 'vacuoles' in $P$. longicornis) should be seen much like those described in tick mid-gut by Raikhel (1975), and the acidic lysosomal-type vacuoles containing haemoglobin described in the digest cells of B. microplus by Lara et al. (2003). Three different types of residual bodies varying in their cellular contents, form and within-cell location are known in the midgut cells of ticks (Raikhel 1978). Can these also be seen in mesostigmatids?

Enzyme histochemistry allows the probing of digestive processes (Tatchell 1964). One can relate the complement of digestive enzymes to the predatory nature of mites-Gorgol and Barabanova (1979). Phosphatases are linked histochemically with the absorption of food (Rothstein et al. 1953; Mathers 1973). As insects are one of the main prey of predatory mesostigmatids and are rich in proteins and lipids (Walter et al. 2017), mite digestive fluids are expected to be rich in proteases and lipases. Cysteine proteases are important in spider mite feeding - Santamaría et al. (2015a). Acidic phosphatase in vacuoles is a classic marker of lysosomal processes involved with vacuoles (Boyer 1971). This will be looked for in the early stages of digestion in P. longicornis.

Akimov and Starovir (1977) point out that as each gut cell in A. andersoni goes through one cycle, morphological and functional changes are linked primarily to the state 
of 'nutrient granules' (aka stainable vacuoles) which enlarge and disintegrate. Vacuolar dynamics will thus be key in understanding what is happening in prey processing by $P$. longicornis. Alkaline phosphatase is a classic marker of fluid transporting epithelia (such as in araneid coxal glands-Gabe (1968); and copepod R-cells—Goyffon and Martoja 1983). Increased activity in cell border microvilli has been implicated in protein absorptions in argasid tick digestion (Tatchell 1964) and tetranychid gut-mediated water balance (McEnroe 1963b). The activity is lost once absorption finishes and blood digestion beginsTatchell (1964). Alkaline phosphatase will be looked for in P. longicornis. Non-specific esterases [known in arachnids-Pollak 1966, including in ticks-Matsumoto (1974)] are an indicator for general cellular activity as well as a marker of explicit esteritic catabolic action (often against pesticides-Schöneich 1970). Therefore their localisation too will be used to explicitly test what is happening during the early stages of prey ingestion and food handling by $P$. longicornis.

Much comparison to parasitic argasid physiology will be made as they face the same challenges in feeding and digestion which predatory pergamasids do (albeit that they are blood feeders and $P$. longicornis is not). They need to: take in a large amount of food with respect to their size usually within a few minutes to 1 hour; 'drop off' the feeding opportunity; concentrate the ingestion markedly by the discharge of a large volume of watery fluid from the coxal organ either during or after the meal; digest the meal promptly; lose excretory material; and, be ready for the next feed and its prompt digestion-yet be resistant to starvation.

\section{Objectives of the experiment}

This comprehensive paper, as the last part of a wide-ranging investigation of trophic adaptations to life in this predatory soil pergamasid, uses light microscopy of serial sections from a 'pulse-chase' experiment to quantify, monitor and numerically model the changes in mid- and hind-gut cells during feeding and digestion after a single prey meal (Fig. 1). The experimental concept of this paper is that matching the histological form of the gut epithelium and the kinetics of cellular changes to those of changes in the lumenal contents of the gut (from Bowman 2017b), and to that of excretion (from Bowman 2017a), informs the function of the gut cells appearing at particular times in $P$. longicornis after a single prey meal 'pulse' (Fig. 2, upper). Such quantitation also allows explicit comparison to tick physiological results.

Intentionally from the outset, the pergamasid gut epithelium and its cells are described 'hypothesis-free' simply by their gross form and shape. That is:

- a simple squamous epithelium formed of 'thin' flat cells (Fig. 2, lower),

- a simple cuboidal or simple columnar epithelium formed of 'square' prismatic cells (Fig. 2, lower),

- a stratified or transitional pseudo-stratified squamous/cuboidal/columnar epithelium formed of tessellated irregularly 'jigsaw-piece' shaped cells (Fig. 2, lower),

- 'free' cells floating in the lumen,

- 'clavate' cells noticeably protruding bulb-shaped into the lumen,

rather than by any a priori assumption from the literature that micro-histology necessarily reliably informs function. 


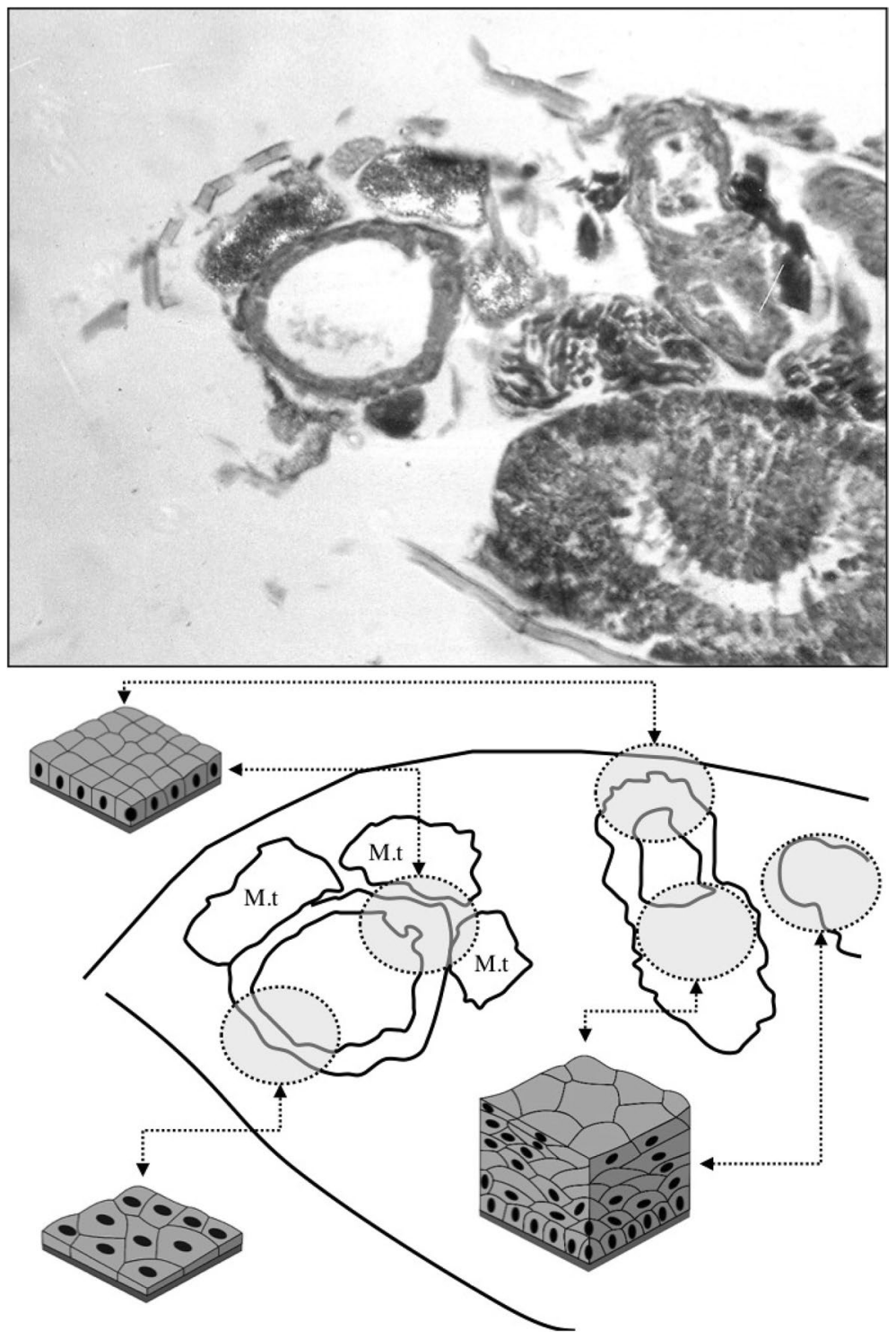

Fig. 2 Tissue types in gut of Pergamasus longicornis. Upper: transverse section (TS) through idiosoma of male mite 5 min after the start of feeding showing mesenteron dorso-centrally and expanded posterodorsal caecum laterally (with beginnings of wispy grey granular prey material within lumen). Stained with Mallory's Triple Stain. Note glistening birefringent guanine granules in Malpighian tubules. Reproductive system to lower right. Lower: line drawing schematic at same scale. M.t = Malpighian tubule (convoluted around caecum). Grey dotted areas highlight different tissue types as examples. Lower left $=$ simple squamous epithelium of flat 'thin' cells. Upper left = cuboidal epithelium of 'square' cells (columnar on further growth over time). Lower right $=$ stratified epithelium of 'jigsaw-piece' like cells (transitional pseudo-stratified on further development over time). Inset tissue type diagrams under Creative Commons Licence from Wikipedia 
To avoid confusion, cell types are not given functional names. Rather, conjectures and attribution to a specific physiological role then relies on the rigorous application of detailed argument. These arguments variously deploy the Bradford-Hill (1965) criteria of: temporal relationship; strength; dose-response relationship; consistency; plausibility; consideration of alternative explanations; experiment; specificity and coherence; all as a structured means of scientific rationality. In taking this approach I follow the philosophy of Rudin and Hecker (1979) who described the functional morphology of the mid-gut of the mosquito Aedes aegypti during blood digestion using both the morphometrics of the stomach epithelium and physiological correlates during the first blood meal (as well as detailing the ultrastructure-Hecker and Rudin 1981). Typical pergamasid gut cell shapes and their relative sizes are shown in Fig. 3.

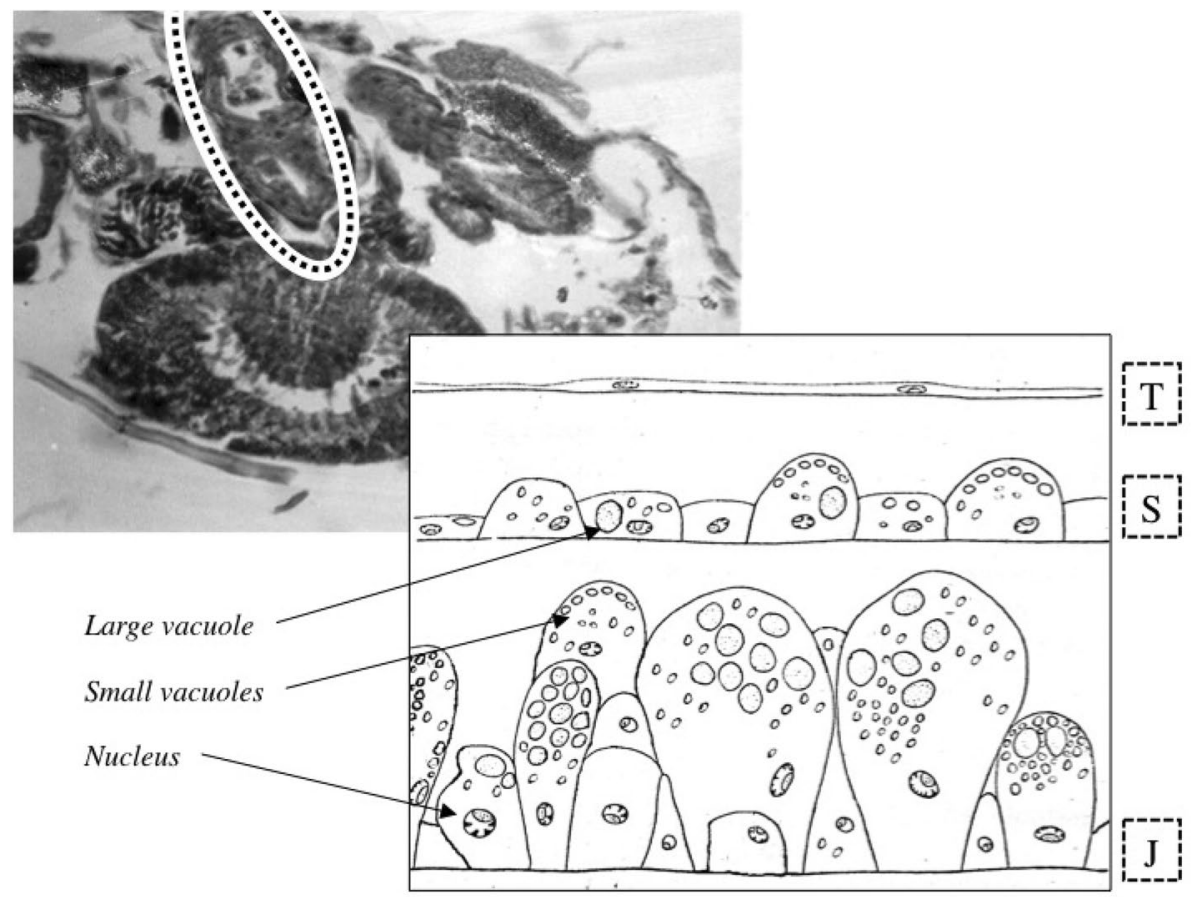

Fig. 3 Tissue types in gut epidermis of Pergamasus longicornis-typical cell forms under vacuolation. Upper left: transverse section (TS) through idiosoma of male mite 5 min after the start of feeding showing mesenteron dorso-centrally (circled by dotted line) comprised of Square and Jigsaw-like cells. Note expanded posterodorsal caecum with almost occluded lumen laterally to the right. Stained with Mallory's Triple Stain. Note glistening birefringent guanine granules in Malpighian tubules. Reproductive system ventrally. Lower right: composite line drawing schematic of cellular epithelium of "Thin cells", "Square cells' and 'Jigsaw cells' with vacuoles ('unwrapped' from circular nature of caecum into a flat picture to show relative sizes and shapes of gut cells. Diagram part extracted and heavily amended from Grandjean and Aeschlimann (1973) CElsevier with permission) simply to illustrate the three pergamasid tissue categories in Fig. 2. $\mathrm{T}=$ thin cells (squamous epithelium) included in diagram for relative size comparison-see Fig. 2, lower left. $\mathrm{S}=$ square cells (columnar/cuboidal epithelium)—see upper left this figure. $\mathrm{J}=$ jigsawlike tessellated cells in pseudo-stratified transitional epithelium-see upper left this figure. Vacuoles at typical relative sizes to nuclei 
So, the pedagogy is:

- the expansion of the pergamasid gut overall, the appearance of a simple squamous epithelium and a gut lumen being present over time $\rightarrow$ that is indicative of ingestion occurring,

- the observed changes in the shape of gut cells on the appearance of a columnar epithelium $\rightarrow$ that is indicative of food absorption occurring,

- the presence of vacuoles (Fig. 3, lower right) over time $\rightarrow$ that is indicative of food digestion (whether being via extracellular enzyme secretions or arising from intracellular uptake) is occurring,

- the relative balance of small and large vacuoles (Baker 1977) $\rightarrow$ that is evidence of the scale of intracellular activity,

- the disproportionate loss of cell epithelium compared to gut lumen for that gut size over time $\rightarrow$ that is evidence of cell degeneration occurring,

- the loss of lumen, the appearance of a pseudo-stratified epithelium, and gut contraction over time $\rightarrow$ that is indicative of egestion occurring and subsequent fasting.

These measures will all be assessed kinetically. A posteriori the final explanation of what might be happening in $P$. longicornis must be appropriate over all levels of observation (i.e., consilient over any scale). Consilience of physiological staging in determining any digestive 'age' grade during feeding to fasting is powerful evidence that any explanation is well founded rationally (for example see the use of heme and protein levels in ticks-Reid and Boid 1984). So, epithelial evidence of pergamasid digestion should be contemporaneous with any observed changes of ingested food (Bowman 2017b), and, epithelial evidence of egestion should be contemporaneous with observed changes in waste contents (Bowman 2017a, b).

Thus this paper in particular seeks quantitatively:

- to order the likely cellular transitions and model their kinetics,

- to relate the appearance of types of cells to the lumenal changes in imbibed prey material reported in Bowman (2017b),

- to link tissue and cell changes to the micro-anatomical changes in gut status reported in Bowman (2014),

- to look for clear evidence of intracellular digestion, and if "Die intrazellulär verdauenden Darmteile sind histologisch gleichartig" (Schlottke 1934a) or not?

- to use enzyme histochemistry to verify what is happening in the early stages of feeding (i.e., before the 'digestive switch' at $2 \mathrm{~h}$ ),

- to investigate the relationship of gut cell changes to the appearance of refractive grains in the lumen as described in Bowman (2017b):

- as such, do the cell changes match the previous schema of 'hidden' egestion (Bow$\operatorname{man} 2017 \mathrm{a})$ ?

- and in particular is there any evidence of cell degeneration to produce this lumenal egestive fraction (sought by Bowman 2017a)?

In doing so, this paper will specifically: 
(a) Confirm or refute that full starvation in P. longicornis is after 2 weeks rather after the 3 weeks reported in Bowman (2017b).

(b) Check for evidence for whether digestion is triggered by:

- maximal gut stretching — estimated as 3.5 min post start of feeding (hypothesis (i) from Bowman 2017b), or

- maximum gut expansion-estimated at 10 min post start of feeding (the favoured hypothesis (ii) from Bowman 2017b), or

- stopping (the act of) feeding — estimated at 56-96 min after start of feeding (hypothesis (iii) from Bowman 2017b).

(c) Check for a 'digestive switch' around $2 \mathrm{~h}$ and an 'egestive' switch around $4 \mathrm{~h}$ (Bowman 2014, 2017b),

(d) Recheck if food elimination is slower in the anterior gut compared to the posterior parts (i.e., the antithesis of hypothesis (iv) in Bowman 2017b),

(e) Check if cellular processes are 'stiff', and how stiff they are compared to gut expansion/ contraction (Bowman 2014), Malpighian processes (Bowman 2017a), and lumenal content processing (Bowman 2017b),

(f) Check 'hypothesis (viii)' from Bowman (2017b) as to just what is a good indicator of starvation in this arachnid,

via a critical assessment of all cell types and their vacuolation quantitatively over time. These will be denoted as 'Check (a)' through to 'Check (f)' in the "Discussion"for easy cross reference.

A kinetic comparison to a further reanalysis of earlier tick results will be made and confirmatory investigations proposed. Finally a functional synthesis is attempted of the gut dynamics during feeding to fasting in P. longicornis (including kinetically modelling the overall gut expansion/contraction data from Bowman 2014). Although an 'assemblyline' arrangement of special organs as in other arthropods (Yonge 1954a) is impossible for arachnids (because the sequence of digestive and absorptive activities is essentially carried out within the vacuoles of single cells-Mitchell 1970), statistical modelling is done in order to verify which anterior or posterior parts of the gut tissues are FIFO (first-in-first-out 'conveyor belt') processes, which are LIFO (last-in-first-out) 'push-pop-stack' processes, or which are liver- or hepatopancreatic-like 'deep storage' depots (i.e., LILO last-in-lastout). In doing so a final determination of what might be the best estimate for the total feeding cycle time in P. longicornis ('hypothesis (vi)' from Bowman 2017b) is made (currently thought to be a little over 52.5 h, i.e., over twice that of phytoseiids studied in the thencalled USSR).

An attempt is made to infer assimilation efficiency in $P$. longicornis and together with an assessment of what starvation actually means for this predator, a unified explanation of all the results from a whole series of $P$. longicornis papers is drawn together.

\section{Materials and methods}

Pergamasus longicornis mites were collected by hand or extracted with a Tullgren apparatus from leaf litter sampled at a variety of deciduous woodland sites in Cumbria, Berkshire, Buckinghamshire, Hertfordshire and Merseyside, UK, in January 1978-March 1979. Species determination used Berlese 1906; Bhattacharyya 1963; Hirschmann 1969; 
Micherdziński 1969 and Karg 1971. Mites were kept individually at indoor room temperature and $>90 \% \mathrm{RH}$ throughout. Mites were starved for 1 week (168 h) to ensure a physiological tabula rasa and then fed individually one final instar larva of the fruit fly Drosophila melanogaster (vestigial wing strain). All mites commenced feeding within 30 min of being offered prey. At 28 distinct pre-specified log-spaced elapsed times from the commencement of feeding, a total of 34 mites were destructively fixed in ice-cold $\left(4^{\circ} \mathrm{C}\right)$ freshly prepared 'Susa', washed and dehydrated through graded isopropyl alcohol, into benzene and double embedded in methyl benzoate/celloidin and paraffin wax under vacuum at $62-64{ }^{\circ} \mathrm{C}$. Sections were taken at $7 \mu \mathrm{m}$, cleared with xylene, rehydrated, stained with Mallory's Triple Stain (acid fuchsin/aniline blue/Orange G-Pantin 1948), re-dehydrated and mounted in DePeX. Transverse sections (TS-Fig. 1) were used so as to be able to estimate comparative surface areas for different gut regions straightforwardly.

\section{Assessments}

Micro-anatomical epithelial status was assessed by inspecting cells in each of 15 gut regions (for abbreviations see Fig. 4) being classified temporally under Nomarski interference phase contrast light microscopy as: thin; square; jigsaw-piece (i.e., irregularly shaped and tesselate, often with indistinct inter-cell borders); Clavate; or, floating Free. Thin cells indicated a simple squamous epithelium (see Fig. 2). Square cells indicated a simple cuboidal or columnar epithelium (see Fig. 2). Jigsaw-piece cells indicated a transitional stratified or pseudo-stratified epithelium (see Fig. 2). Then each of those Thin, Square or Jigsaw cells were characterised temporally as having: none; small; or, large usually pink-orangered stained vacuoles. Note clear areas of cytoplasm that were non-staining and not clearly membrane limited were not scored as 'vacuoles' (unlike Akimov and Starovir 1977 and Starovir 1979a). Rather vacuoles herein match the 'nutrient granules' scored by the Soviet workers. For each cell type/vacuole combination, a 3 point prevalence score across the sections was used as: Many $=2$, Some $=1$, None $=0$. An attempt was made in this analysis to utilise colour changes in the stained vacuoles (as Till 1961 did in Rhipicephalus appendiculatus) but no other cytological features were examined. Given the vagaries of histological staining, colour change is poor evidence to ascribe definitively the contents of vacuoles without 'on-section known' standards and should only be used indicatively.

Relative occurrence of characteristics over sections is assumed to measure relative amounts overall. Given the thickness of histological sections taken $(7 \mu \mathrm{m})$, vacuole profile scores are indicative of the actual diameter of the food inclusions predominant in the gut cells as the great majority of the vacuole/food inclusions would be included intact. Unlike in the TEM of very thin sections, scoring is not strongly biased and stereological adjustment is unnecessary. At any one time point and anatomical location the gut tissue may show simultaneous evidence of all three epidermal types. Scores were not initially normalised by gut (or cell) volume to derive a surrogate of concentration. No attempt at examining the gut for cilia (Rohde and Oemick 1967) nor detailed musculature (Prasse 1967) was made. However, sections were examined for evidence of chitinous walls or membraneous/ fibrous envelopes in the gut. Salivary glands, coxal glands and haemocoelic fat body-like tissues ('dorsal connective tissues') were not explicitly scored. No distinction of vacuoles as intracellular absorbed food depots versus secretions was made in the scoring in this histological study. 


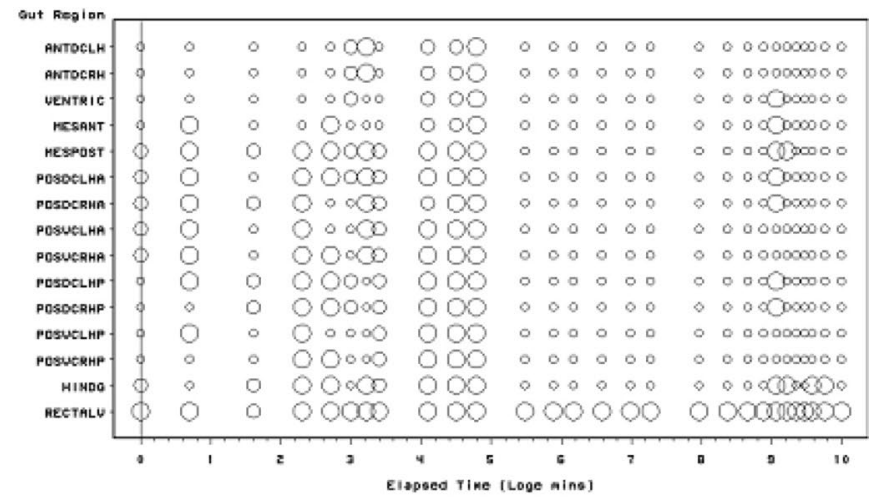

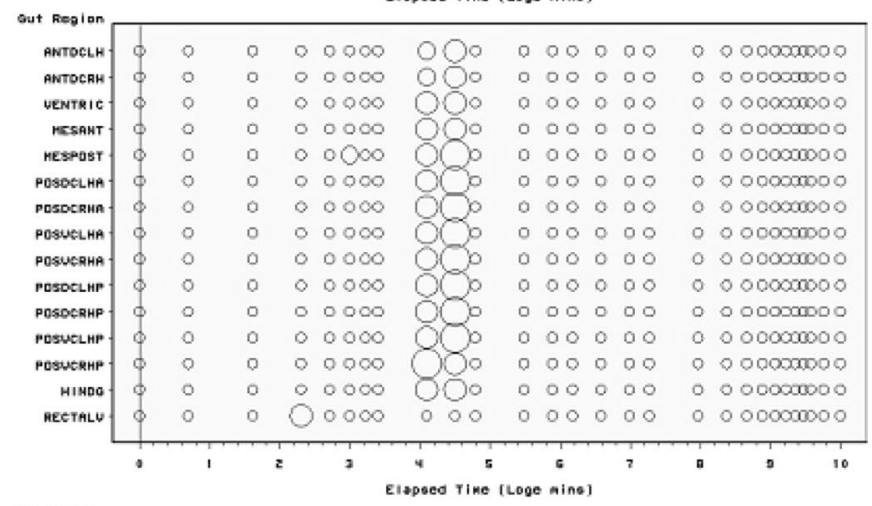

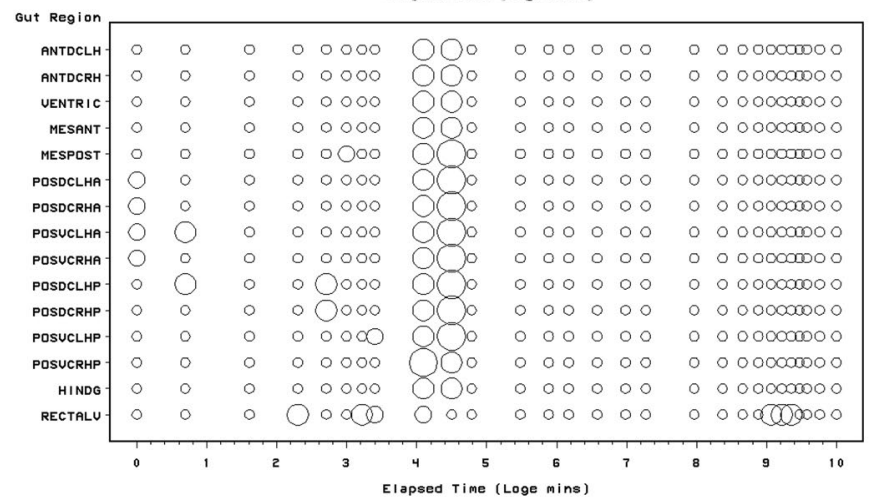

Fig. 4 Schematic of Pergamasus longicornis data for 'Thin' gut epidermal cell status in each gut region (ordered anterior to posterior) as: present (large circles), or, Absent (small dots). Intermediate size circles represent ambiguity over replicates. Time is from the commencement of feeding and is on a natural logarithmic scale. Grey line is at the $0 \mathrm{~min}$ commencement of feeding. Associated gut region abbreviations: anterodorsal caecum LH = ANTDCLH; Anterodorsal caecum RH = ANTDCRH; Ventriculus = VENTRIC; Mesenteron anterior = MESANT; Mesenteron posterior = MESPOST; Posterodorsal caecum LH anterior = POSDCLHA; Posterodorsal caecum RH anterior = POSDCRHA; Posteroventral caecum LH anterior = POSVCLHA; Posteroventral caecum RH anterior = POSVCRHA; Posterodorsal caecum LH posterior $=$ POSDCLHP; Posterodorsal caecum RH posterior $=$ POSDCRHP; Posteroventral caecum LH posterior $=$ POSVCLHP; Posteroventral caecum RH posterior $=$ POSVCRHP; Hind gut $=$ HINDG; Rectal vesicle $=$ RECTALV. Left-hand and right-hand denotation was done by looking from the dorsal side anteriorly. Upper: status; Middle: small vacuole score. Lower: large vacuole score 


\section{Data management}

Due to collecting constraints, no distinction was made between the 16 male mites sampled at times after the start of feeding: $0 \mathrm{~min}, 2,2 \times 5,10,25,60,90 \mathrm{~min}, 6 \mathrm{~h}, 12,48,96,168$, 192, 240, $288 \mathrm{~h}$ and the 18 female mites sampled at times after the start of feeding: $0 \mathrm{~min}$, $15,2 \times 20,2 \times 30,60,90 \mathrm{~min}, 2 \mathrm{~h}, 4,8,18,24,72,120,144,216,336$ h). Seasonality of digestive activity such as in Varroa jacobsoni (Akimov and Barabanova 1990) was not considered. Month and year of collection (Bowman 1985) was not adjusted for. Genders are pooled as in Bowman (2014). A maximum of $336 \mathrm{~h}$ was chosen based upon the scaling calculations of Adolph (1949) applied to the maximum investigation time of phytoseiids used in previous Soviet studies (cf. $48 \mathrm{~h}$ ) and the food disappearance time of laelapids from Kanungo (1964) - allowing for the larger size of pergamasids using body sizes from: Schweizer (1961); Stammer (1963); Evans and Till (1966) and Karg (1971).

All data were coded, stored and manipulated in Access 97, Excel 2011, FTN 77 or SAS 6.12. Diagrams were produced in SAS 6.12, Excel 2011, GIMP 2.8, Graphis 2.7.3 and Powerpoint 2011. Elapsed time from zero was log transformed (time $>0$ ). When necessary for displays $t=0$ was given a log value of 0 . A logarithmic scale for elapsed time was chosen upon the simplest assumption that physiological changes represent first order rate processes (Lister et al. 1988) — this is the same assumption as in the models of Sabelis (1990). Natural $\left(\log _{e}\right) \operatorname{logarithms}$ were used throughout and labeled $\ln$.

\section{Statistical analysis}

Bayesian modelling of the probability $(P)$ of a specific cell type used OpenBugs 3.23 with non-informative Jeffreys priors (Congdon 2001). A burn-in of 30,000 updates followed by summaries of a further 30,000 updates was used. The notation 'hat' is used to mean 'estimated value'. The log odds ratio comparing anterior sections (subscript $=1$ ) to posterior sections (subscript $=2$ ) was calculated as $\ln \left[\frac{\pi_{1}}{\left(1-\pi_{1}\right)} \cdot \frac{\left(1-\pi_{2}\right)}{\pi_{2}}\right]$ where $\pi$ is the estimated probability of the occurrence of specific cell type in the gut. This statistic is asymptotically Normally distributed as $N\left(\ln (\right.$ odds ratio $\left.), \sigma_{\ln (\text { odds ratio })}^{2}\right)$ under the null of no distinction between the two regions. A two-sided $z$-test $(p<0.05)$ is used herein. Density plots were only to be prepared for significant results.

At each of the $i=34$ time points, the histological (but not the histochemical) data herein plus those of Bowman $(2014,2017 \mathrm{a}, \mathrm{b})$ were combined. The resultant $k=785$ characters (covering gut and Malpighian tubule, lumenal and cellular states-initially scored 0, 1, or 2) were rescaled (to a 0 to 1 probability range) to yield a matrix $\mathbf{A}$, such that the elements $A_{i, k}$ were the $k$ th normalised state for the $i$ th time point. Missing data ( $\equiv 1.7 \%$ ) from damaged sections were replaced by modal values (Sneath and Sokal 1973). Inter-point distance $\left(d_{i, j}\right.$ values $\left.=[0 \ldots 1]\right)$ over the $k$ normalised character states between two time-slices $i=1 \ldots 33$ and $j=(i+1) \ldots 34$ was calculated by a symmetric Jacquard style measure:

$$
d_{i, j}=\frac{1}{\sum_{k=1}^{k=785}\left(w_{k}\right)} \cdot \sum_{k=1}^{k=785}\left(w_{k} \cdot\left|A_{i, k}-A_{j, k}\right|\right),
$$

where $w_{k}=$ weights for the $k$ th character and $|\ldots .$.$| is the absolute value operator. The$ weights for this city-block metric were defined as $\frac{1}{q}$ where $q=1$ or the number of sub-character states scored underneath that character (if it had any) - following the philosophy of Proctor and Kendrick (1963) without the additional plus 1. These weighted 'band pass 
filter' distances in turn give proportionately more weight to the similarity of cellular characteristics than to tissue structural/cell type similarities than to micro-anatomical similarities. These were then used to fit the observed time point sequence to a circle by minimising the total of the step-wise distances $\left(\sum_{i, j}\left(d_{i, j}\right)\right.$ including 'back-to-the-start') between the entire time-points set-via an exhaustive computational search over all $\frac{1}{2} \cdot(34$ !) re-orderings of the $i, j=1 \ldots 34$ sections.

Taking the 34 time points and the 14 gut sections (ignoring RECTALV) above (with the scores from Bowman 2014, 2017a, b) for: Thin cells (with and without small, large vacuoles); Square cells (with and without small, large vacuoles); Jigsaw-like cells (with and without small, large vacuoles); clavate cells; free cells; total vacuoles; gender; gut expansion/contraction; Malpighian tubule expansion/contraction; Malpighian tubule guanine; lumen presence/absence; membranous material in lumen; granular material in lumen; refractive granules in lumen; globular material in lumen; other material in lumen; plus each lagged by 1-3 time points (first observation carried back); a data set of all scores $(0,1,2$ or 0,1$)$ was constructed for physiological ordination. This was analysed using SVD decomposition of the correlation matrices of individualised divergences against the reference starved state of $t=0$ (Bowman 2015).

\section{Kinetic estimation}

Linear kinetic modelling of the Bayesian posterior mean estimates was done by exponential stripping (Kirkup and Sutherland 1988) on an arithmetic time scale using Excel 2011. Kinetic modelling excluded the rectal vesicle as a part of the gut. A first order differential empirical model for the probability of a cell type occurrence at any time $t$ in the observed 'current' gut compartment was used via the directed output input relationship:

$$
P[t]=a_{e l} \cdot e^{b_{e l} \cdot t}-a_{i n} \cdot e^{b_{i n} \cdot t},
$$

where: $P$ denotes the probability of a specific cell type observed at time $t$, the subscript in denotes input, and the subscript $e l$ denotes elimination ( $\equiv$ output). For the rate constant $b_{e l}<0$ the effective size of $a_{e l}$ declines away with a half-life $t_{\frac{1}{2}}=\frac{\ln (2)}{b_{e l}}$. If there was no input then $a_{e l}=P[0]$. The stripping process traditionally fits late occurring data under the assumption that elimination is dominating and that input has essentially completed. Then subtracts the fitted values (assuming this elimination) from the original data to form 'adjusted' data. Then fits the early occurring adjusted data when input is dominating and elimination only just under-way in order to estimate the input parameters. So, the contribution $a_{i n} \cdot e^{b_{i n} . t}$ represents in a positive sense the first order 'elimination' $\left(b_{i n}<0\right)$ from a notional previous or up-stream compartment being now input into the observed down-stream gut cell one. In this example $\hat{a}_{i n}=\hat{a}_{e l}=e^{\left(\max \left[\hat{P}_{t}\right]\right)}$ since stripping should ensure a crossing or knot point for the functions at the peak of $P[t]$. The half-life of the input function is $\frac{\ln (2)}{b_{i n}}$. All physiological phenomena are assumed to be present from $t=0$ after the start of feeding, so all other matters being equal, the linear superposition principle ensures that the shape of any profile from compartment to compartment spreads out as the signal passes along a series of compartments arranged one-after-another. The initial signal thus becomes 'blurred' and attenuated (per force diminished in extent) - even if an interlinked network of compartments are assumed. For the purposes of illustration in some instances input models assuming no elimination were estimated directly from the rise in unadjusted data over specific time periods and through particular maximum values. This approach is appropriate when the half-lives of absorption and elimination are different by orders of magnitude. Kinetic models in some 
cases also used data from Bowman (2014, 2017a, b) to provide insight in teasing out possible cellular functions. Vacuolation over all cell types was summarised and kinetically modelled in a similar fashion. Small vacuoles were considered as lesser versions of large vacuoles, large vacuoles as greater versions of small ones.

\section{Histochemistry}

Enzyme histochemical assays followed Pearse (1960) on neutral formalin-fixed sections. Non-specific esterase was assayed by simultaneous diazo coupling of $\alpha$-napthyl acetate with Fast Blue RR in neutral pH conditions. Acidic phosphatase was assayed in acid conditions by simultaneous diazo coupling of $\alpha$-napthyl phosphate with Fast Garnet GBC. Alkaline phosphatase was assayed in alkaline conditions by simultaneous diazo coupling of $\alpha$-napthyl phosphate with Fast Blue B. Location and depth of colour deposits were assessed subjectively on a 6 point comparative scale from zero, trace,,,++++++ , to ++++ (by amount not colour). The gut by region including the pre-oral cavity, as well as: the dorsal connective tissues (akin to tick 'fat body' - Coons et al. 1990); the salivary glands; the coxal glands plus nephrocytes; and, the Malpighian tubules were all scored enzyme histochemically.

\section{Results}

The oesophagus in $P$. longicornis passes through the brain and rises to join the mid-gut from below. No evidence was seen of the oesophagus projecting into the gut lumen as a 'valve' (as in Tyroglyphids-Oboussier 1939; Prasse 1967; Legendre 1967; or, as in hydrachnids Pollock 1898). However, no evidence of regurgitation (as in argasids-Moorehouse 1975) especially into the oesophagus was observed either. The general layout of the mid-gut in P. longicornis is as found in parasitids (Winkler 1888). The gut overall was pale in colour (i.e., without any dark brown 'liver' appearance reported by Mégnin 1876) like the opaque white of that in Gamasus (Eugamasus) immanis-King (1913). The cells had a feebly staining micro-granular cytoplasm as in amblyseiids (Akimov and Starovir 1977). The mid-gut wall consisted of epithelial cells resting on the basal lamina or membrane propria sheathed by muscle fibres. No evidence of the sorting of prey material into different gut epithelial regions (such as a 'crop' or a special absorptive zone-Yonge 1954a) was observed. As in A. andersoni (Akimov and Starovir 1977) there were no morphological differences between the cells of the mesenteron and those of the diverticula-even though the caeca are corrugated [like in uropodines-Michael (1894), macrochelids-Butler (1964) and in starved Amblyseius spp.-Akimov and Starovir (1976)]. As in bdellids (Alberti 1973) and unlike as in Mitopus morio (Phillipson 1961), there was no histological difference between ventricular and caecal cells. The caecal (diverticula) cell types appeared the same in all central mid-gut regions just as in ticks.

As in P. persimilis (Starovir 1973) considerable functional changes in the epithelial cells of the gut occurred within minutes of the start of feeding. Three clearly differentiable types of mid- and hind-gut epithelial form and constituent cells were observed in P. longicornis:

- 'thin' cells with either no vacuolar material; with small vacuoles; or, with large vacuoles; forming a narrow pavement epithelium (see Fig. 4);

- 'square' cells (again with no vacuolar material; with small vacuoles; or, with large vacuoles); forming a strong cuboidal epithelial layer (see Fig. 5); 


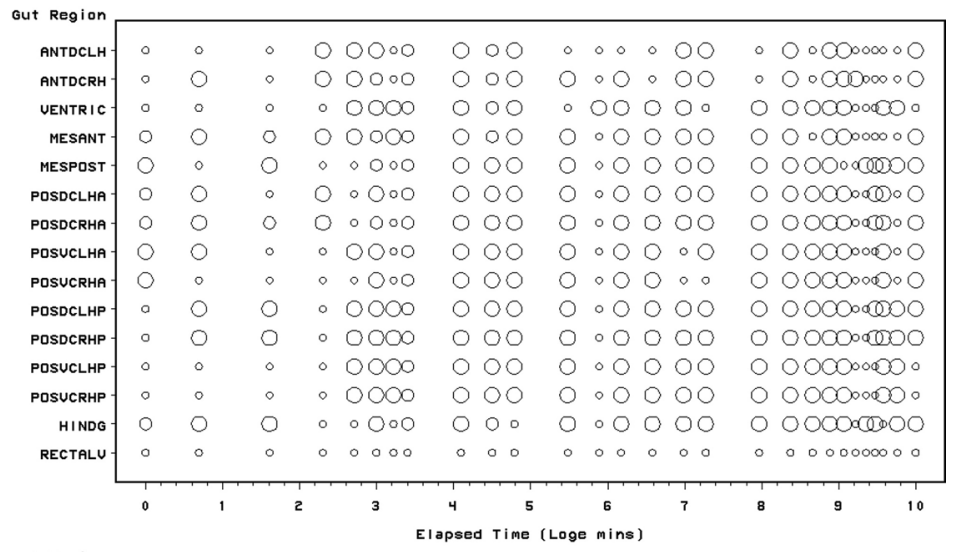

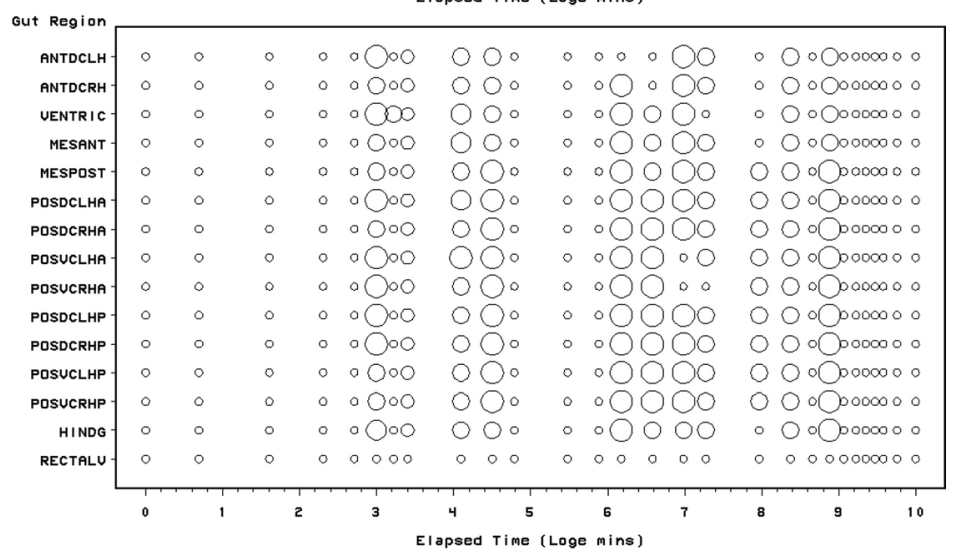

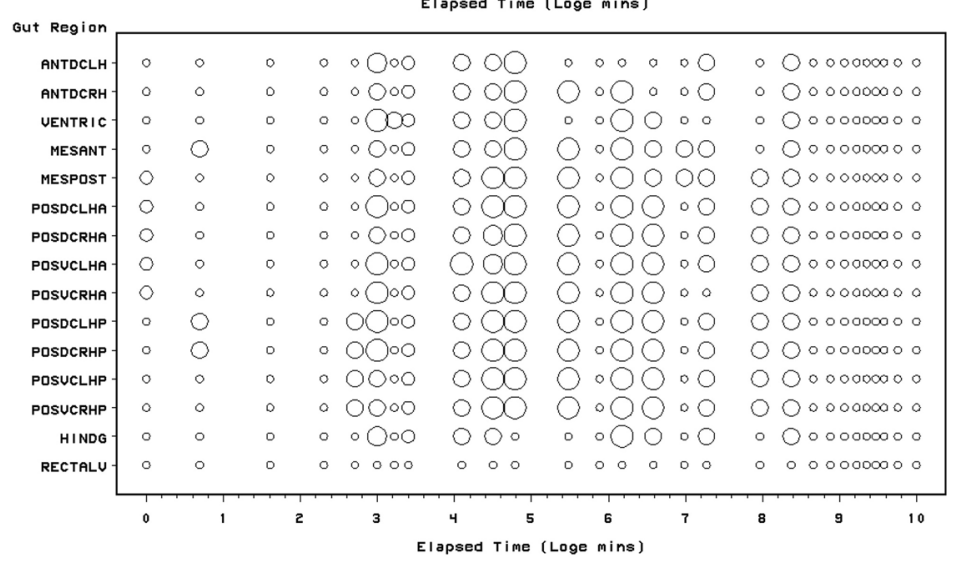

Fig. 5 Schematic of Pergamasus longicornis data for 'Square' gut epidermal cell status in each gut region (ordered anterior to posterior) as: present (large circles), or, absent (small dots). Intermediate size circles represent ambiguity over replicates. Time is from the commencement of feeding and is on a natural logarithmic scale. Associated gut region abbreviations as in Fig. 4. Upper: status; Middle: small vacuole score. Lower: large vacuole score 
- 'jigsaw-piece' cells (irregularly-shaped tessellated cells with no vacuolar material; with small vacuoles; or, with large vacuoles); in close packing often encroaching onto the lumen forming a transitional pseudo-stratified epithelium (see Fig. 6).

Each of these cell types gradually waxed and waned over feeding to fasting and occurred together at various times and locations in different proportions. Summary profiles for 'thin', 'square' and 'jigsaw-piece' cells are shown in Figs. 7, 8, 9. They are discussed in detail below. Just as in scorpions (Goyffon and Martoja 1983), fasting induces a relative decline in the volume of diverticular tissue and a decrease in vacuolation-all indicating a disappearance of apparent food reserves. No midgut cells obviously containing guanine or micro-crystalline residual bodies or lipofucsin vesicles were seen with light microscopy in P. longicornis. This is consilient with the intestine having the lowest nitrogen and phosphorus levels amongst the organs of ticks-Stepanchenok-Rudnik et al. (1971).

No obvious histological differentiation of the cells in the hind-gut compared to rest of gut was found (confirming similar results in tyroglyphids-Oboussier 1939). The endodermic hind-gut epithelium appeared and behaved just like the mid-gut epithelium-there being no evidence of hind-gut specialisation as found in terrestrial isopods (Coruzzi et al. 1982) either. A very thin flat epithelium, confirming its partial endodermic origin (Legendre 1968) was present in the rectal vesicle (as in the rectum of tyroglyphids-Oboussier 1939). The gut epithelium was also examined and scored for any chitinous wall (Fig. 10, upper); and any extra membranous wall (Fig. 10, lower). This rectal vesicle epithelium was the only part of the P. longicornis gut to show such augmentation by chitinous and membraneous components, confirming its partial ectodermic origin (Legendre 1968). No obvious peritrophic matrix (Peters 1968) or continuous material-containing membrane (van der Borght 1966; Rudsinska et al. 1982; Zhu et al. 1991) was found anywhere in the pergamasid mid- or hind-gut (however Bowman (2017b) recounts membrane fragments in the lumen of the rectal vesicle). Any lipids within cells known in ticks for exampleKitaoka (1961a); Caperucci et al. (2010), or used as overwintering stores as in tetranychids-McEnroe (1970), would have been lost by virtue of the fat-dissolving solvents during histological sample preparation and could not be scored.

Gut cell vacuolation looked just like that in the gut wall of Argas persicus (see Fig. 11). All parts of the gut changed in size over time. No obvious stem cells as in ticks (Tarnowski and Coons 1989) were observed. Any mechanism of cell replacement, after any exfoliation of cells, from regenerative cells (El Shoura 1988) or by division was not examined in this study. No undifferentiated reserve cells (Agbede and Kemp 1985) were recorded in P. longicornis - so how replacement cells (Tarnowski and Coons 1989) presumably from mid-gut stem cells or 'regenerative cells' (El Shoura 1988) for the next meal are produced if required is not discussed. Perhaps the general 'digestive' gut cell simply divides when quiescent in the unfed/starved pseudo-stratified epithelium? Of course cell duplication of any of the histologically scored cell types may actually be occurring in $P$. longicornis but without counting each individual cell over time this cannot be definitively claimed. Rather the schema below in the "Discussion"simply relies upon cells apparently becoming bigger and smaller and the epithelium changing in overall form. The uneven physiological ageing/ senescence of the gut epithelium as in ticks (Repkina 1976) is confirmed.

Two other cell types were occasionally observed and scored: 'clavate' cells (Fig. 12, upper) protruding deep into the gut lumen; and, 'free' cells (Fig. 12, lower) apparently 


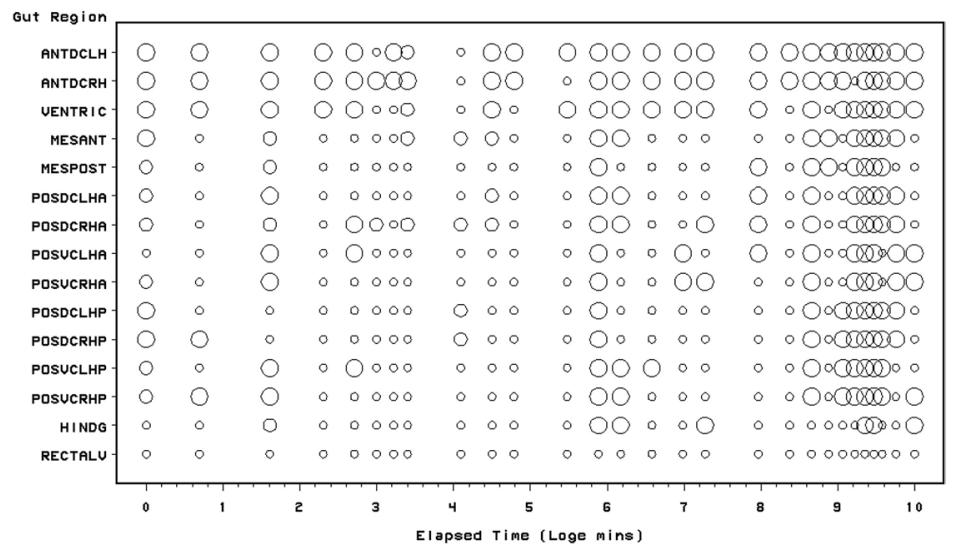

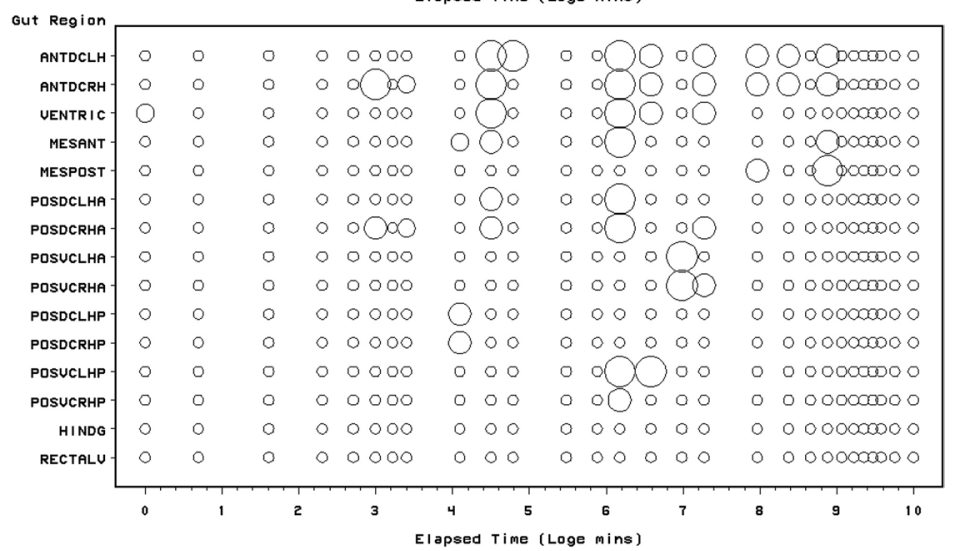

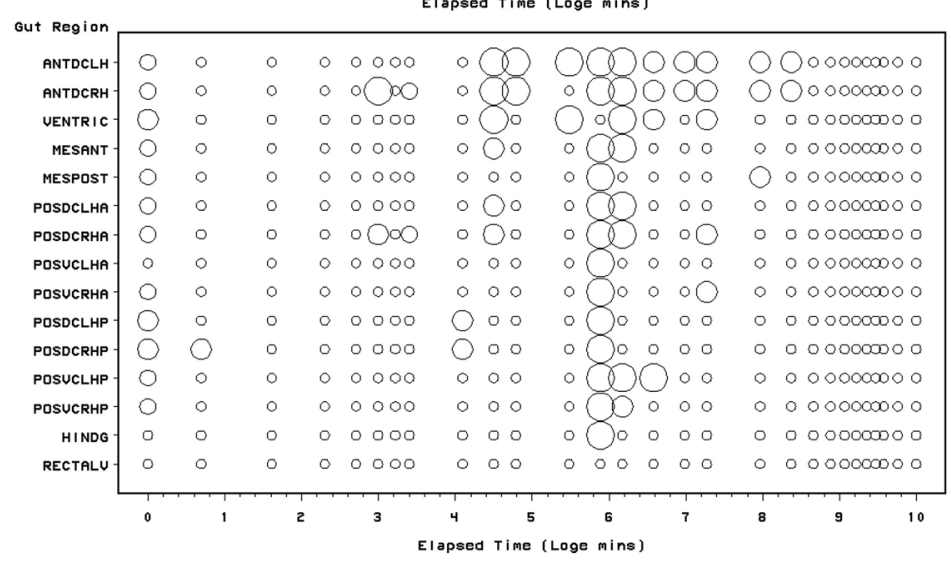

Fig. 6 Schematic of Pergamasus longicornis data for 'Jigsaw-piece' like irregularly tessellated gut epidermal cell status in each gut region (ordered anterior to posterior) as: present (large circles), or, Absent (small dots). Intermediate size circles represent ambiguity over replicates. Time is from the commencement of feeding and is on a natural logarithmic scale. Associated gut region abbreviations as in Fig. 4. Upper: status; Middle: small vacuole score. Lower: large vacuole score 


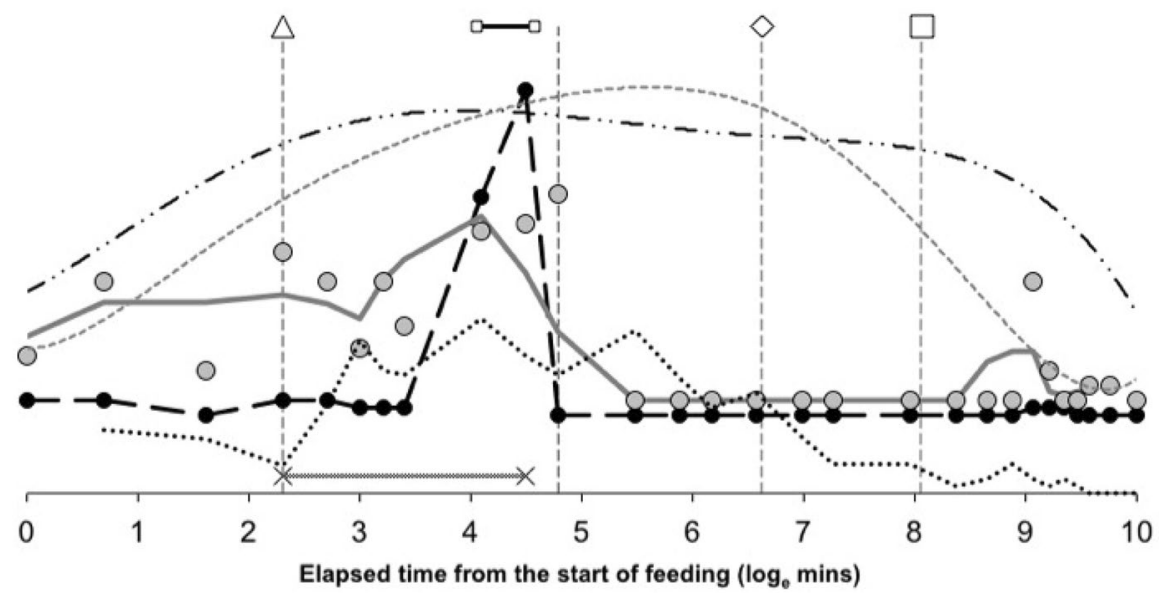

Fig. 7 Time-course of simple squamous epithelium (thin cells) in gut of Pergamasus longicornis during feeding, digestion, egestion and excretion. Thin cells (solid grey circles, grey solid third order moving average trend line). Average (in thin cells) of small or large vacuole status, black circles and dashed black line. The vacuolated thin cells are only present in number late on in prey feeding; at the latter stages of the presence of coxal droplets, and at the time of maximum gut expansion and lumenal presence. They disappear immediately at the end of feeding $(2 \mathrm{~h})$. Gut expansion/contraction mean score from Bowman (2014) - no symbol, upper heavy dashed grey (sixth order polynomial) trend line. Lumen presence/absence mean score from Bowman (2017a) — no symbol and black dashed dotted smoothed (fifth order polynomial) trend line. Marbled lower grey line is granular contents in the lumen (Bowman 2017b). Solid hashed line ending with $\mathrm{X}=$ presence of 'coxal droplets' (Bowman 2014). Square indicates worse-case total feeding cycle time 52.5 $\mathrm{h}$ based upon Bowman (2014) modelling gut expansion/contraction, thereafter is egestion. Triangle indicates best estimate of initial gut filling time $10 \mathrm{~min}$ (Bowman 2014). Diamond indicates best estimate of time of initial gut emptying $12.5 \mathrm{~h}$. Small open squares joined by black line at top are mean feeding times for males and females (Bowman 1987a). Grey vertical dotted line centrally is at the 120 min optimal knot position between net gut-filling and net gut-emptying (Bowman 2014). Y-axis is arbitrary but does infer relative amounts between plotted points within each line

floating in the lumen. Not many cells were clearly and unequivocally projecting into the gut lumen as observed in uropodids by Ainscough (1960). Free cells had a 'bead-let' organisation internally. At four time points $(30 \mathrm{~min}, 8 \mathrm{~h}, 12 \mathrm{~h}$, and 6 days after the commencement of feeding), the basal part of the rectal vesicle epithelium appeared to have clear 'holes' in it in various places occasionally. It is not clear what this was. This could have been fat deposits lost during the course of slide preparation. No kinetic modelling of this (nor of the clavate nor of the free cells) was undertaken due to the paucity of data. Under light microscopy, no gut cells with clear indigestible refractive or birefringent residues within them (as in flatworms and scorpions-Schlottke 1934b), nor cells packed with faecal material (as in chiggers-Mitchell and Nadchatram 1969) were observed. Cell with indisputable contents of refractive grains (Bowman 2017b), or with minute bluish-black granules or purplishblue inclusion bodies dispersed through them as Till (1961) recounts in ticks, were not observed.

\section{Statistical analysis}

In a $\log$ (odds ratio) test, there was no formal evidence (nor a consistent trend) for an anterior:posterior axis in the occurrence of: thin cells (mean $=-22.95$, sd $=14.03)$; thin 


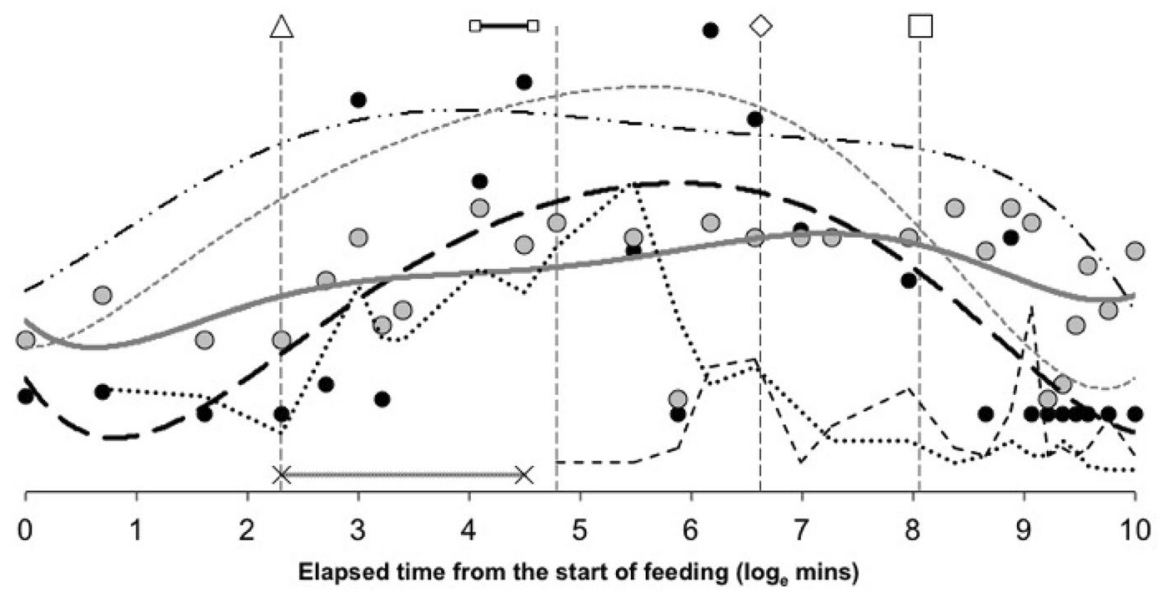

Fig. 8 Time-course of simple cuboidal/columnar epithelium (square cells) in gut of Pergamasus longicornis during feeding, digestion, egestion and excretion. Square cells (solid grey circles, grey solid sixth order polynomial trend line). Average (in square cells) of small or large vacuole status, black circles and dashed black line. The square vacuolated cells are only present in number late on in prey feeding; at the latter stages of the presence of coxal droplets, and at the time of maximum gut expansion and lumenal presence. They disappear around the end of the total feeding cycle of $52.5 \mathrm{~h}$ (Bowman 2014). Lower marbled line is total of granular, globular and other material in the gut lumen. Dashed lower line to the right starting $2 \mathrm{~h}$ after feeding is total of refractive grains and membraneous faecal material in the gut lumen. Symbols and extra lines as in Fig. 7

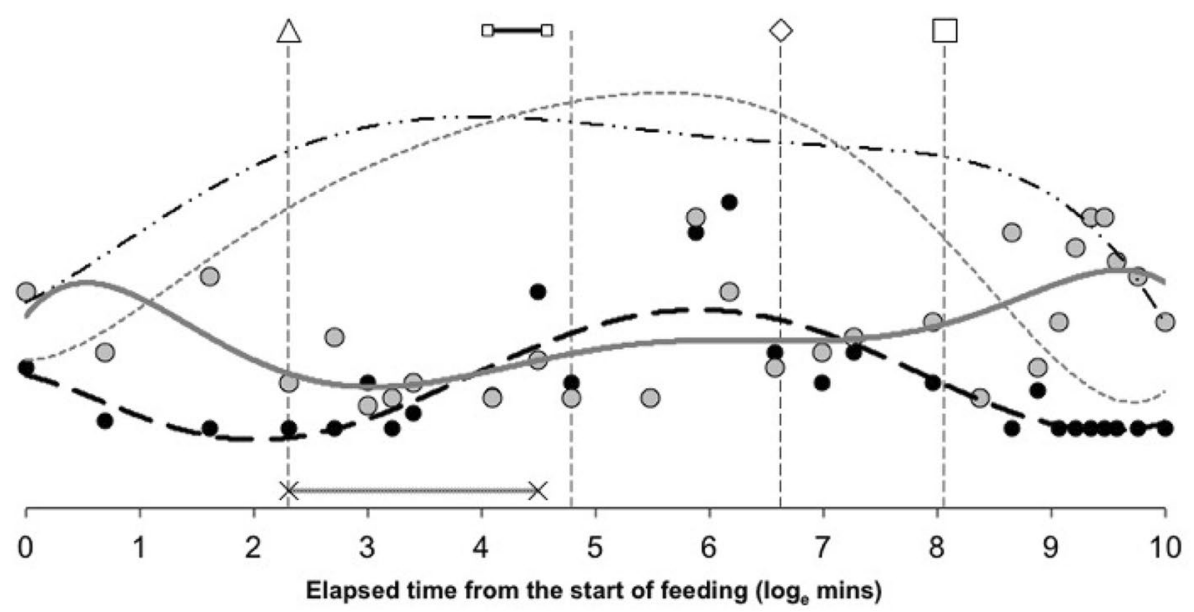

Fig. 9 Time-course of pseudo-stratified/transitional epithelium (jigsaw-piece like irregularly tessellated cells) in gut of Pergamasus longicornis during feeding, digestion, egestion and excretion. Jigsaw cells (solid grey circles, grey solid sixth order polynomial trend line). Average (in jigsaw cells) of small or large vacuole status, black circles and dashed black line. The pattern resembles that of square cells (Fig. 8) but of less amplitude. Symbols and extra lines as in Fig. 7 


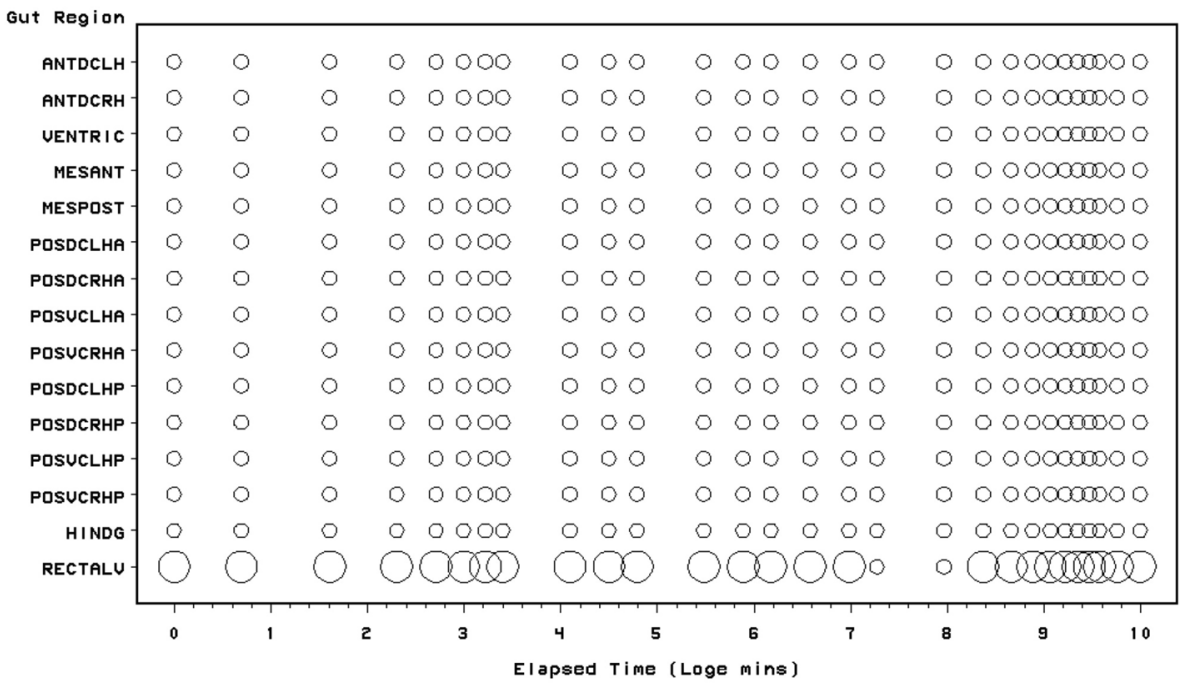

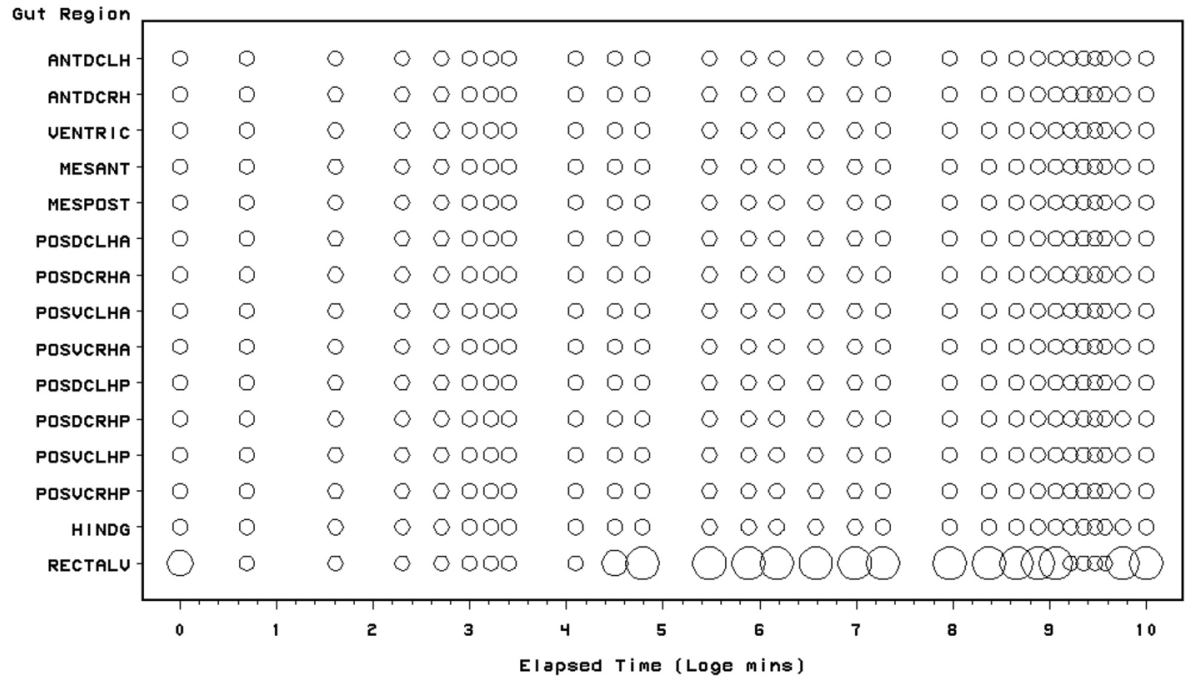

Fig. 10 Schematic of Pergamasus longicornis gut wall status for each gut region (ordered anterior to posterior) as: present (large circles), or, absent (small dots). Intermediate size circles represent ambiguity over replicates. Time is from the commencement of feeding and is on a natural logarithmic scale. Associated gut region abbreviations as in Fig. 4. Upper: chitinous wall. Note: only the rectal vesicle has a chitinous wall. Lower: membraneous wall. Note: only the rectal vesicle has a clear acellulate membranous wall

cells with small vacuoles $($ mean $=18.85$, sd $=15.9)$; thin cells with large vacuoles (mean $=8.365$, sd $=15.44)$; square cells (mean $=-26.75$, sd $=11.22)$; square cells with small vacuoles $($ mean $=1.391$, sd $=13.95)$; square cells with large vacuoles (mean $=-4.779$, sd $=12.72)$; jigsaw-piece cells $($ mean $=62.64, \mathrm{sd}=10.74)$; jigsaw-piece cells with small vacuoles $($ mean $=39.79$, sd $=13.68)$; jigsaw-piece cells with large vacuoles $($ mean $=$ 39.07 , $\mathrm{sd}=13.5)$; clavate cells $($ mean $=23.64$, $\mathrm{sd}=14.04)$; free cells $($ mean $=5.703$, $\mathrm{sd}=$ $15.48)$; vacuoles $($ mean $=9.59$, $\mathrm{sd}=10.69)$. No consistent staining colour difference was 

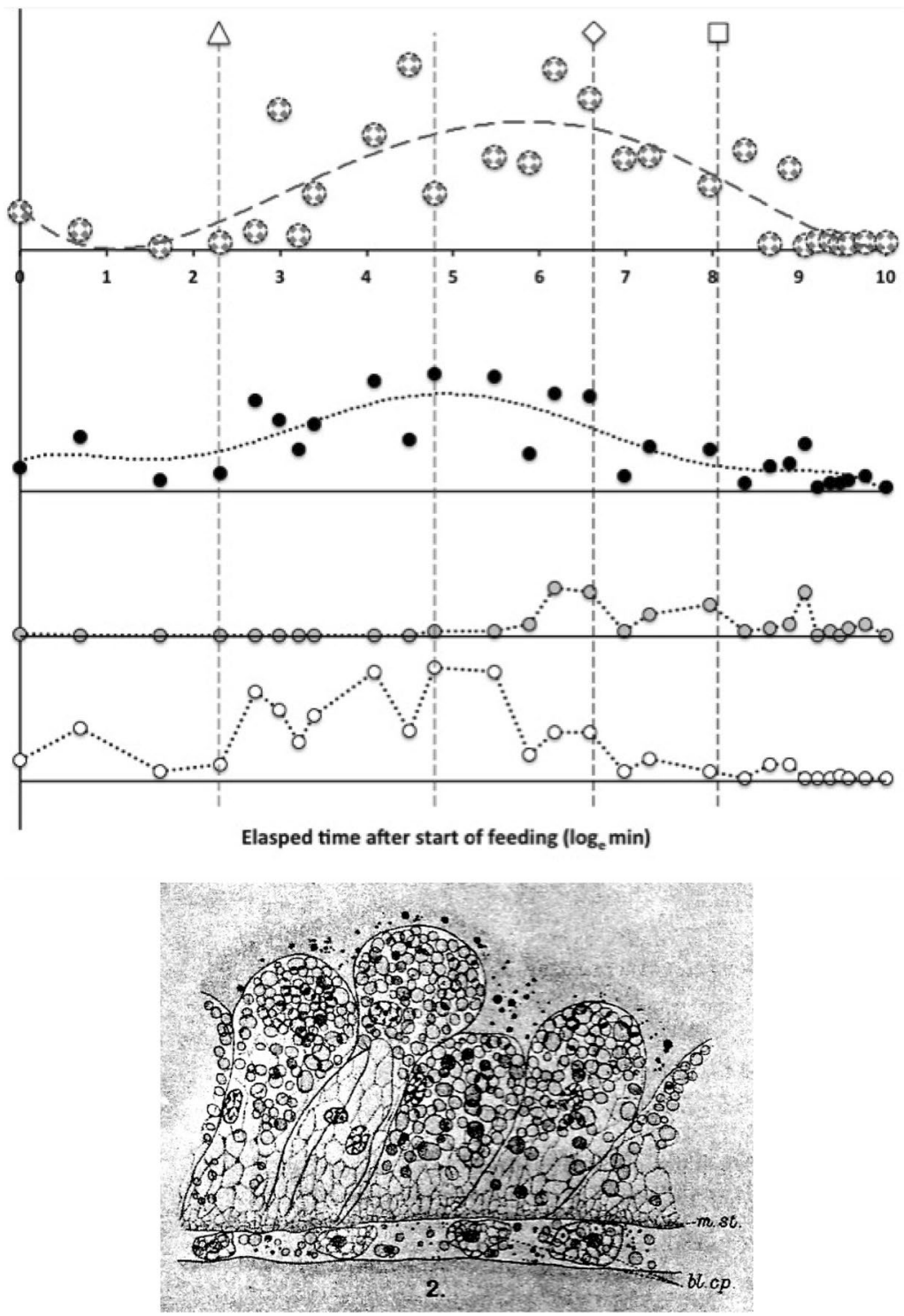

Fig. 11 Relation of vacuolation to lumen contents (taken from Bowman 2017b) in Pergamasus longicornis from feeding to fasting. Upper box. Top track: fitted probability of vacuoles in gut epithelial cells-cellular white and grey circles, grey dashed sixth order polynomial trend line. The pattern resembles that of square cells (Fig. 8) and jigsaw cells (Fig. 9) showing whole epithelium vacuolates coherently but at slightly different times over cell types depending upon preponderance of each cell type. Lower tracks: black circles with dotted sixth order polynomial trend line-total occurrence of globular, granular and refractive granular lumenal material. Grey filled circles and dotted line-occurrence of refractive granular material in gut lumen. White open circles and dotted line-total occurrence of granular and globular lumenal material. Symbols as in Fig. 7. Lower figure. Typical appearance of a vacuolated anactinotrichid acarine gut cellfrom Argas persicus (Robinson and Davidson 1913-1914) @Cambridge University Press with permission. Fed pergamasid mid-gut epithelia look like this 


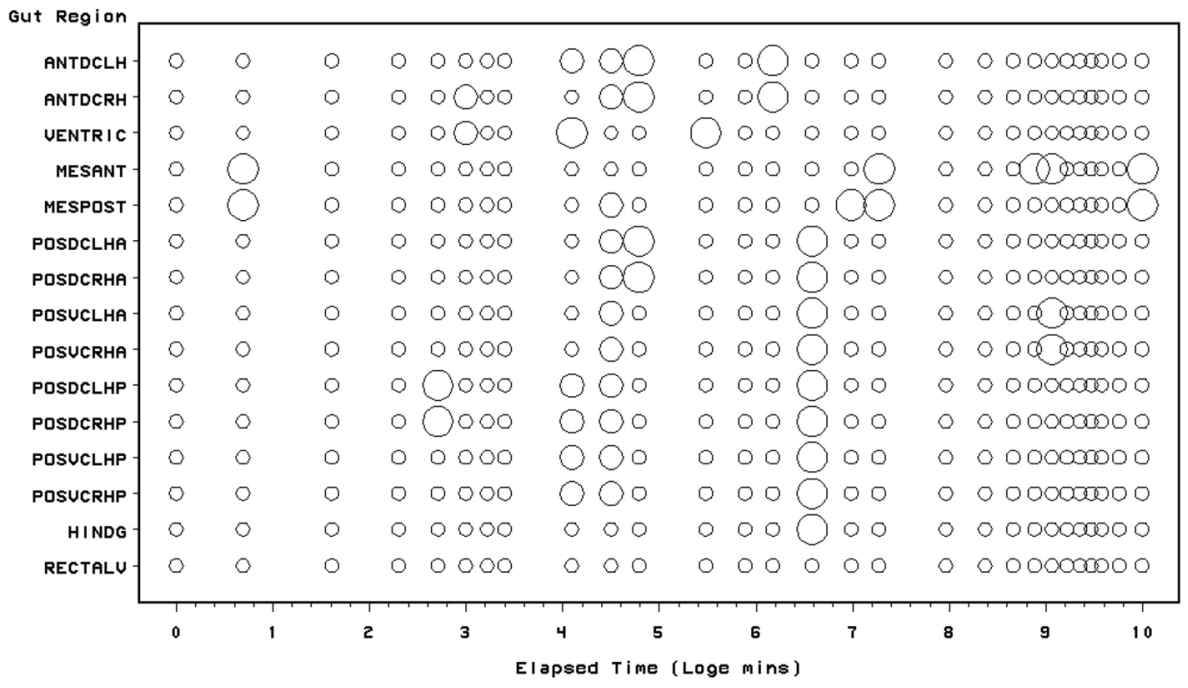

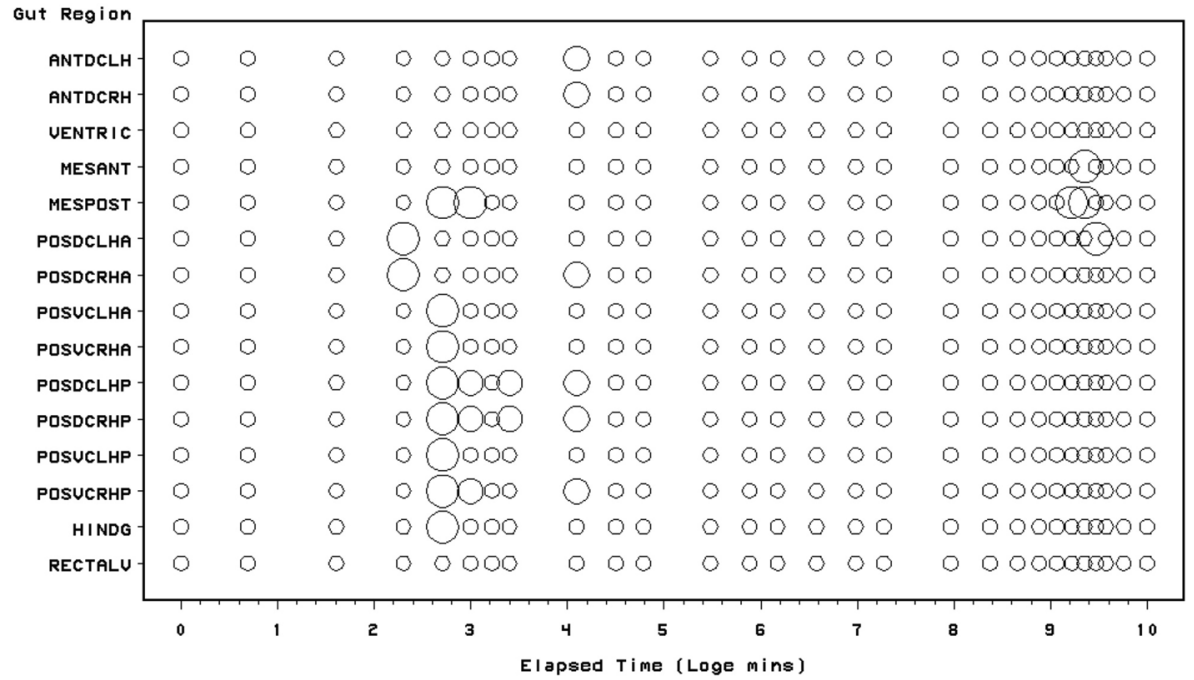

Fig. 12 Schematic of Pergamasus longicornis gut cell status for each gut region (ordered anterior to posterior) as: present (large circles), or, absent (small dots). Intermediate size circles represent ambiguity over replicates. Time is from the commencement of feeding and is on a natural logarithmic scale. Associated gut region abbreviations as in Fig. 4. Upper: 'Clavate' cells. Note two pulses: one around 1-2 h; the other, at $12-24 \mathrm{~h}$ post start of feeding. Lower: 'Free' cells. Note early peak

seen between large and small vacuoles (results not shown). Cellular changes were synchronous over the whole epithelium (bar the rectal vesicle) as in Androlaelaps casalis (Starovir 1981a). Note that the log odds ratio for an anterior: posterior axis in the gut expansion/ contraction data from Bowman (2014) was: mean $=-35.87$, $\mathrm{sd}=8.915$ and is also not significant.

The pergamasid gut epithelium was heteromorphic consisting of sets of fundamentally homomorphic cells. The mapping of the sequence of overall histological results onto a 
Fig. 13 Statistical analysis. Upper: minimum spanning circle mapping of inter-point distances $\left(d_{i, j}\right)$ between the complete histologically scored character data at each time point from feeding to fasting in Pergamasus longicornis. Uses all cellular data herein plus that from Bowman (2014, 2017a, b). Distance around circle is reciprocally weighted Jacquard similarity index between adjacent sequentially displayed points, confirming four phases. (D) $=$ digestion phase. (I, E) = ingestion, egestion/excretion phase. $(\mathrm{O})=$ osmoregulatory ('coxal droplet') phase. Physiological time course for partially overlapping phases should be read anticlockwise $(\mathrm{I} \rightarrow \mathrm{O} \rightarrow \mathrm{D} \rightarrow \mathrm{E})$. Labels are in min or $\mathrm{h}$ (see Materials and Methods). Dot is used to denote different replicates for clarity. Greyed out label for $6 \mathrm{~h}$ is at peak of production of globular material in the gut lumen (Bowman 2017a). Greyed out label for aberrant result at $366 \mathrm{~h}$ is during starvation. Middle: left: scores plot from divergences SVD (black circles $=$ reference $t=0$. Middle: right: loadings plot from SVD. Small open circles $=$ lag terms. Note that to the right and down direction are: black squares $=$ cell characteristics; grey squares = granular and globular food; crosses = expansion/contraction and lumen score. Note to the left and up direction are: $\mathrm{X}=$ other and membraneous material in lumen; grey stippled square $=$ refractive grains; open circle $=$ Malpighian tubule expansion and guanine. Middle: central: $\operatorname{Ln}($ time $)$ contours over SVD scores plot showing time passes anticlockwise (dark $\rightarrow$ pale, black $\equiv$ initial starved state, white $\equiv$ starving. Lower: scores plot from SVD with line showing time course of changes for each gut region overlain on anatomy from Bowman (2014). Note 'echo' or extra cycle (cf. Fig. 18, middle) in posterior gut caeca-'slow' digestion before fasting/starvation?

minimum spanning circle is shown in Fig. 13, upper confirming a single cycle of tissue changes from feeding upon a single prey. No short-term cytological cycling was observed. SVD of the results show an anticlockwise transit of each gut section in terms of its characteristics compared to the starved basis reference (Fig. 13, middle). This is driven by first cellular and lumenal contents characteristics (horizontal transit on feeding/ingestion), then egestive and excretory characteristics (vertical transit) as the idiosoma eventually re-equilibrates back to the starved state. Evidence for a second physiological 'echo' or possible 'slower' intracellular digestive phase on 'tougher' prey material is shown by Fig. 13, lower posteriorly.

\section{Kinetic estimation}

Kinetic model results can be found in Table 1. Fitting simultaneous absorption (input) and elimination (output) models gave poor results. Fitting separate appearing kinetics (with no simultaneous disappearing kinetics) and separate disappearing kinetics (with no simultaneous appearing kinetics) — each going through appropriate maximum values $[\max (P)]$ —gave stable summaries. Note, inputs were fitted to $(\max (P)-P)$ to ensure the correct functional shape. Kinetic models could not be satisfactorily fitted to the occurrence of jigsaw-piece, clavate or free cells and are summarised with a narrative. A stiff epidermal system is indicated (see "Discussion").

\section{Histochemistry}

Enzyme histochemical results for the early stages of feeding are shown in Tables 2, 3, and 4. As pointed out by Lake and Ellis (1976), quantifying these changes is useful as long as absolute values are not being considered, realising that it is only the difference over time or location that is being quantified. Non-specific esterase activity was generally diffusealthough tissues could show some positive globular localisations (in vacuoles?). Within any one locality of activity, generally esterase activity increased posteriorly if present. This agrees with digestion being greater at the caecal tips in $P$. persimilis Starovir (1973). Gut cell esterase activity, if present, was patchy and sporadic in discrete local sub-oval 'hot 

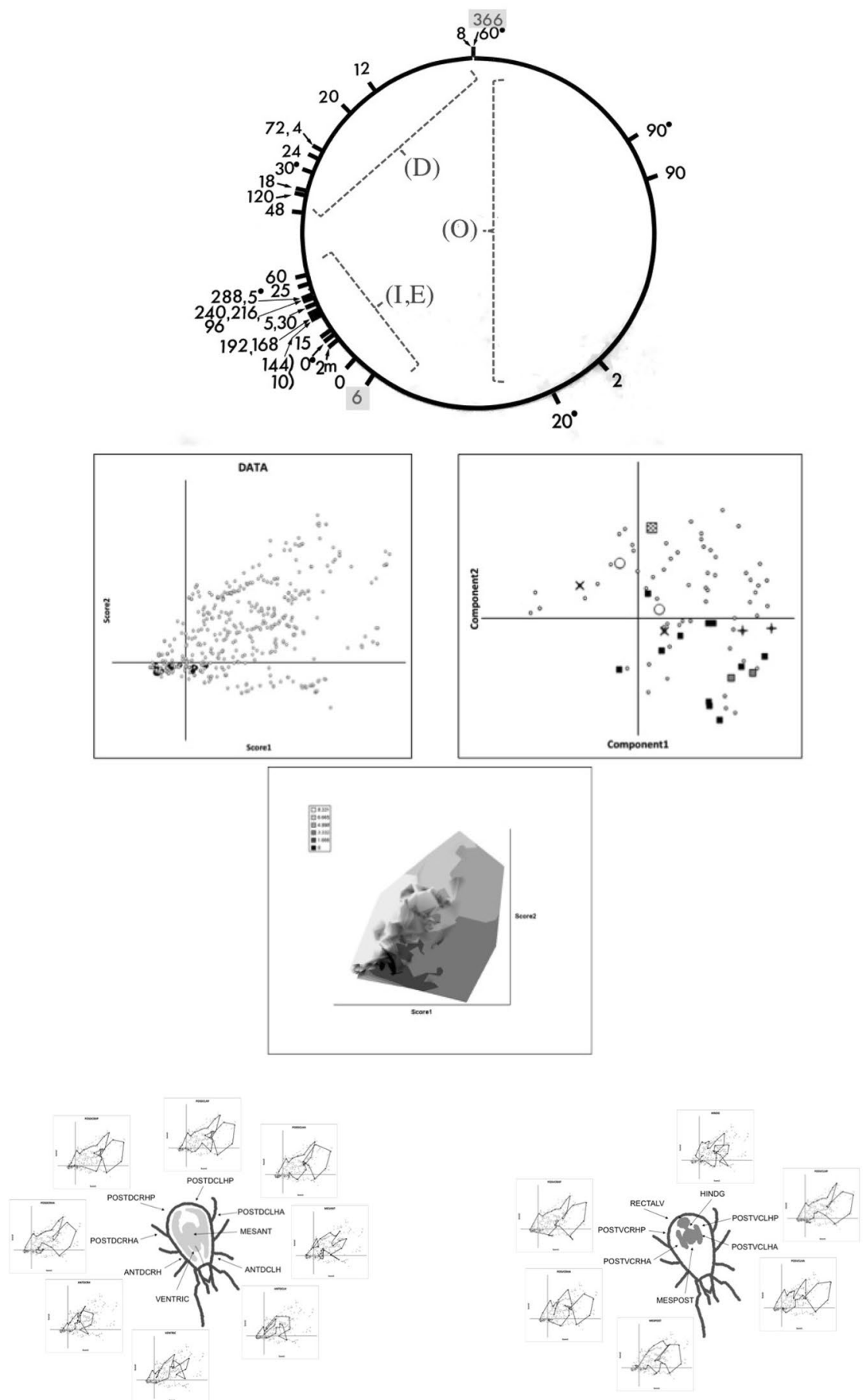
Table 1 Estimated digestive status half lives in each separate kinetic phase of processing for various tissue and cell phenotypes during feeding to fasting in Pergamasus longicornis

Tissue phe- Kinetic phase of cellular process ${ }^{\ddagger} \quad \hat{t}_{1 / 2} \quad 3^{*} \hat{t}_{1 / 2}(\equiv 87.5 \%) \quad 5^{*} \hat{t}_{1 / 2}(\equiv 96.875 \%)$
notype

Squamous (thin cells)

\section{Appearing}

Overall 0-2 h

Slow 0-30 min

Fast 30-120 min*

Disappearing

Instant $90 \mathrm{~min}-6 \mathrm{~h}$

Columnar (square cells)

\section{Appearing}

From 0 to $2 \mathrm{~h}$

Disappearing

From 2 to 10 days

Vacuolation

Appearing

From 0 to $90 \mathrm{~min}$

From 0 to $90 \mathrm{~min}$ (ant.)

From 0 to $90 \mathrm{~min}$ (post.)

Disappearing

From $8 \mathrm{~h}$ to 6 days

From 8 h to 6 days (ant.)

From 8 h to 6 days (post.)

From 8 to $366 \mathrm{~h}^{* *}$

From 8 to $366 \mathrm{~h}$ (ant.)**

From 8 to $366 \mathrm{~h}$ (post.)**

Gut overall ${ }^{\dagger}$

\section{Expansion}

From 5 to $30 \min ^{\dagger \dagger}$

$50.6 \mathrm{~min}$

$2.5 \mathrm{~h}$

Contraction

From 2 to $366 \mathrm{~h}$

"Lumenation"

\section{Appearing}

From 2 to $18 \mathrm{~h}$

Disappearing

From 2 to 9 days

$\begin{array}{lll}31.8 \mathrm{~min} & 1.6 \mathrm{~h} & 2.7 \mathrm{~h} \\ 5.5 \mathrm{~h} & 16.5 \mathrm{~h} & 27.4 \mathrm{~h} \\ 22.9 \mathrm{~min} & 1.2 \mathrm{~h} & 1.9 \mathrm{~h} \\ & & \\ 49.5 \mathrm{~min} & 2.4 \mathrm{~h} & 4.1 \mathrm{~h}\end{array}$

$39.1 \mathrm{~min} \quad 2.0 \mathrm{~h}$

4.9 days $\quad 14.8$ days

24.7 days

$\begin{array}{lll}1.1 \mathrm{~h} & 3.3 \mathrm{~h} & 5.5 \mathrm{~h} \\ 1.2 \mathrm{~h} & 3.5 \mathrm{~h} & 5.9 \mathrm{~h} \\ 1.1 \mathrm{~h} & 3.3 \mathrm{~h} & 5.4 \mathrm{~h} \\ & & \\ 1.0 \text { days } & 3.1 \text { days } & 5.1 \text { days } \\ 1.4 \text { days } & 4.1 \text { days } & 6.8 \text { days } \\ 1.1 \text { days } & 3.4 \text { days } & 5.6 \text { days } \\ 1.7 \text { days } & 5.2 \text { days } & 8.7 \text { days } \\ 2.4 \text { days } & 7.2 \text { days } & 12.0 \text { days } \\ 1.9 \text { days } & 5.7 \text { days } & 9.5 \text { days }\end{array}$

$7.7 \mathrm{~h} \quad 23.2 \mathrm{~h} \quad 38.7 \mathrm{~h}$

4.6 days $\quad 23.1$ days
21.9 days

Note no modelling of jigsaw-piece, free or clavate cells due to paucity of data. For gut 'Lumenation' see Fig. 20 and "Discussion", data from Bowman (2017b)

${ }^{\ddagger}$ Each phase separately fitted to data—simultaneous kinetic models for appearance and disappearance gave inappropriate estimates

*Trimmed to examine consistency and in order to compare to 20-120 min interval in Bowman (2017b)

**Extended to give slowest possible decline based upon data (see text)

$\dagger$ Data and time-windows for kinetic modelling from Bowman (2014)

${ }^{\dagger}$ Trimmed for stable estimates 
Table 2 Non-specific esterase enzyme histochemical results by gut region in Pergamasus longicornis after feeding on a single fly larva

\begin{tabular}{|c|c|c|c|c|}
\hline Time after start of feeding & $0 \min$ & $15 \min$ & $30 \min$ & $2 \mathrm{~h}$ \\
\hline Physiological phase ${ }^{\dagger}$ & $\mathrm{S}$ & I & $\mathrm{O}$ & $\mathrm{D}$ \\
\hline Typical gut cell ${ }^{\ddagger}$ & Jigsaw & Thin & Square & All+vac \\
\hline \multicolumn{5}{|l|}{ CELLS* } \\
\hline Anterodorsal caeca & + & & + & \\
\hline Ventriculus & & + & + & \\
\hline Mesenteron & & & + & \\
\hline Posterodorsal caeca & & + & + & \\
\hline Posteroventral caeca & & + & + & \\
\hline Hind gut & & & + & \\
\hline \multicolumn{5}{|l|}{ Rectal vesicle } \\
\hline Dorsal connective tissues & Trace & ++ & & \\
\hline Salivary gland & ++ & ++ & + & ++ \\
\hline Coxal gland and nephrocytes & + & & Trace & \\
\hline \multicolumn{5}{|l|}{ Malpighian tubules } \\
\hline \multicolumn{5}{|l|}{ LUMEN } \\
\hline Pre-oral cavity & ++ & Trace & + & \\
\hline Anterodorsal caeca & & Trace & Trace & \\
\hline Ventriculus & & Trace & Trace & \\
\hline Mesenteron & & Trace & Trace & \\
\hline Posterodorsal caeca & & Trace & Trace & \\
\hline Posteroventral caeca & & Trace & Trace & \\
\hline \multicolumn{5}{|l|}{ Hind gut } \\
\hline Rectal vesicle & & & & \\
\hline
\end{tabular}

Number of ' + ' indicate comparative visual scale of activity (blank cell $=$ no activity observed). Ingestion (I), osmoregulation (O) and early digestion (D) phases before the 'digestive switch' (at $2 \mathrm{~h}$-Bowman 2014)

${ }^{\dagger}$ See Fig.13. (S) means 'starved' (for 4-10 days)

${ }^{\ddagger}$ See Figs. 4, 5, 6

*Irrespective of type. Feeding stops after 56-96 min (Bowman 1987a). Coxal droplets are formed between 10-90 min (Bowman 2014). Non-specific esterase activity was generally diffuse-although tissues could show some positive globular localisations (in vacuoles?). Within any one locality of activity, generally esterase activity increased posteriorly if present. Gut cell esterase activity, if present, was patchy and sporadic in discrete local sub-oval 'hot spots' of cells. Note pre-oral activity

spots' of high activity cells (often near the basement membrane-as in argasids Tatchell 1964). Only traces were found lumenally. Acidic phosphatase activity was generally diffuse (possibly in Golgi, rough endoplasmic reticulum?) but tissues could occasionally show some positive globular localisations (in vacuoles?). This esterase and acid phosphatase localisation matches that confined to vacuoles in Unionicola intermedia-Baker (1977). Alkaline phosphatase activity was invariably diffuse and when in gut cells was often strongly marginal to the lumen. Unlike in Baker (1977), no globular localisations of alkaline phosphatase activity were found-rather marked lumenal activity was found 
Table 3 Acidic phosphatase enzyme histochemical results by gut region in Pergamasus longicornis after feeding on a single fly larva

\begin{tabular}{|c|c|c|c|c|}
\hline Time after start of feeding & $0 \mathrm{~min}$ & $15 \mathrm{~min}$ & $30 \mathrm{~min}$ & $2 \mathrm{~h}$ \\
\hline Physiological phase ${ }^{\dagger}$ & $\mathrm{S}$ & I & $\mathrm{O}$ & $\mathrm{D}$ \\
\hline Typical gut cell ${ }^{\ddagger}$ & Jigsaw & Thin & Square & $\mathrm{All}+\mathrm{vac}$ \\
\hline
\end{tabular}

Anterodorsal caeca

Ventriculus

Mesenteron

Posterodorsal caeca

Posteroventral caeca

Hind gut

Rectal vesicle

Dorsal connective tissues

$+$

$++\quad+$ Trace

$+\quad++$ Trace

$+\quad++$

$+\quad++$ Trace

Trace ++

Salivary gland

Trace

Coxal gland and nephrocytes

$++++$

Malpighian tubules

+ Trace

LUMEN

Pre-oral cavity

Anterodorsal caeca

Trace

Ventriculus

Trace

Mesenteron

Posterodorsal caeca

Posteroventral caeca

$\begin{array}{cr} & \text { Trace } \\ ++ & \text { Trace } \\ ++ & \text { Trace } \\ ++ & \text { Trace } \\ & \text { Trace }\end{array}$

Hind gut

Rectal vesicle

Number of ' + ' indicate comparative visual scale of activity (blank cell $=$ no activity observed). Ingestion (I), osmoregulation (O) and early digestion (D) phases before the 'digestive switch' (at $2 \mathrm{~h}$-Bowman 2014)

†See Fig.13. (S) means 'starved' (for 4-13 days)

${ }^{*}$ See Figs. 4, 5, 6

*Irrespective of type. Feeding stops after 56-96 min (Bowman 1987a). Coxal droplets are formed between 10 and 90 min (Bowman 2014). Acidic phosphatase activity was generally diffuse although tissues could occasionally show some positive globular localisations (in vacuoles?). At 15 min after the start of feeding acid phosphatase activity in the lumen was associated with imbibed prey material

at $2 \mathrm{~h}$ after feeding (supporting the general schema in Fuzita et al. 2016a, b). Alkaline phosphatase activity was found pre-orally. The starved gut epithelial state in P. longicornis was typified by little enzymatic activity except some acidic phosphatase activity. Validation using another species (Pergamasus septentrionalis) confirms this quiescence on fasting (Table 5). Both acidic phosphatase and esterase was found in the salivary glands of P. longicornis. Esterase activity was also found pre-orally. These results are used in the "Discussion" below. 
Table 4 Alkaline phosphatase enzyme histochemical results by gut region in Pergamasus longicornis after feeding on a single fly larva

\begin{tabular}{|c|c|c|c|c|}
\hline $\begin{array}{l}\text { Time after start of feeding } \\
\text { Physiological phase }\end{array}$ & 0 min & $15 \mathrm{~min}$ & $30 \mathrm{~min}$ & $\begin{array}{l}2 \mathrm{~h} \\
\mathrm{D}\end{array}$ \\
\hline Finsiorogical priase & & & & \\
\hline Typical gut cell ${ }^{\ddagger}$ & Jigsaw & Thin & Square & All+vac \\
\hline \multicolumn{5}{|l|}{ CELLS* } \\
\hline Anterodorsal caeca & & ++ & & Trace \\
\hline Ventriculus & & ++ & & Trace \\
\hline Mesenteron & & +++ & & + \\
\hline Posterodorsal caeca & & ++ & & + \\
\hline Posteroventral caeca & & +++ & & + \\
\hline \multicolumn{5}{|l|}{ Hind gut } \\
\hline \multicolumn{5}{|l|}{ Rectal vesicle } \\
\hline \multicolumn{5}{|l|}{ Dorsal connective tissues } \\
\hline \multicolumn{5}{|l|}{ Salivary gland } \\
\hline \multicolumn{5}{|l|}{ Coxal gland and nephrocytes } \\
\hline \multicolumn{5}{|l|}{ Malpighian tubules } \\
\hline \multicolumn{5}{|l|}{ LUMEN } \\
\hline Pre-oral cavity & & + & & \\
\hline Anterodorsal caeca & & & & ++ \\
\hline Ventriculus & & & & ++ \\
\hline Mesenteron & & & & +++ \\
\hline Posterodorsal caeca & & & & +++ \\
\hline Posteroventral caeca & & & & +++ \\
\hline Hind gut & & & & +++ \\
\hline Rectal vesicle & & & & \\
\hline
\end{tabular}

Number of ' + ' indicate comparative visual scale of activity (blank cell $=$ no activity observed). Ingestion (I), osmoregulation (O) and early digestion (D) phases before the 'digestive switch' (at $2 \mathrm{~h}$-Bowman 2014)

${ }^{\dagger}$ See Fig.13. (S) means 'starved' (for 4-13 days)

${ }^{\ddagger}$ See Figs. 4, 5, 6

*Irrespective of type. Feeding stops after 56-96 min (Bowman 1987a). Coxal droplets are formed between 10 and 90 min (Bowman 2014). At 15 min after feeding - cellular activity strongly in tips and top third of cells marginal to lumen in large localised areas of epidermal protrusions throughout mid-gut. At $2 \mathrm{~h}$ after feeding cellular activity in globular structures (vacuoles?). Lumenal activity was clearly associated with imbibed grainy prey material. Note pre-oral activity

\section{Giant mycetome-like cells}

Giant cells were found in nearly all pergamasids examined (range 0-11 per mite- Table 6). These mycetome-like cells were described in 6 locations in P. longicornis by Bowman (1984a) and called 'Coons cells' after the description of four similar midgut cells in Macrocheles muscaedomesticae by Coons (1978). They comprise of a 'giant cell' usually embedded in the epithelial axil of the mesenteron (with the joining caeca) or near/at the ends of posterior caeca (see Table 6). They could be syncytial in nature. Those described 
Table 5 Enzyme histochemical results by gut region in starved Pergamasus septentrionalis fed on a single fly larva

\begin{tabular}{llll}
\hline Time after start of feeding & $0 \mathrm{~min}$ & $0 \mathrm{~min}$ & $0 \mathrm{~min}$ \\
Enzyme & Non-specific esterase & Acid phosphatase & $\begin{array}{l}\text { Alkaline } \\
\text { phos- } \\
\text { phatase }\end{array}$ \\
\hline
\end{tabular}

CELLS*

Anterodorsal caeca

Ventriculus

Mesenteron

Posterodorsal caeca

Trace

Posteroventral caeca

Hind gut

Rectal vesicle

Dorsal connective tissues

Trace

Salivary gland

Coxal gland and nephrocytes

$+$

Malpighian tubules

LUMEN

Pre-oral cavity

Anterodorsal caeca

Ventriculus

Mesenteron

Posterodorsal caeca

Posteroventral caeca

Hind gut

Rectal vesicle

Number of ' + ' indicate comparative visual scale of activity (blank cell $=$ no activity observed). Starved for 6-14 days

*Irrespective of type. Note zero activity for leucine aminopeptidase (by Fast Garnet GBC diazocoupling) — a marker of intracellular protein digestion. Note zero lipase (by nonanoate/taurocholate reaction) activity also

by Coons (1978) have large numbers of inclusions and two are found in the ventriculus posterodorsally and one at each of the two distal tips of the ' 3 rd caeca' (三 posterodorsal caeca in P. longicornis). What are they?

Various possibilities arise-perhaps they are commensals, symbionts, parasites or pathogens? Nearly 100 years ago, Sig (1930) describes round sporidial parasites in invertebrates. Bacteria as symbionts or pathogens are known in the digestive gut of $D$. gallinaeBaker and Whittington (1980). Jaschke (1933) summarised the symbionts in ixodids. More recently, Chlamydia-like microorganisms have been found in the hepatopancreas cells of spiders (Osaka 1973). These giant mycetome-like cells in P. longicornis may be related to the extra-intestinal groups of micro-organisms reported in soil saprophagous mites (Smrž 2003). They were not associated with obvious signs of histopathology (unlike the detrimental bacterial infections in ticks-Vidomsky et al. 1973). In general aspect they resembled the mycetome in Bdellonyssus/Ornithonyssus bacoti illustrated by Hughes (1952, 1959) which looks like that found in lice (Ries 1931). Their location (posterior mesenteron, 


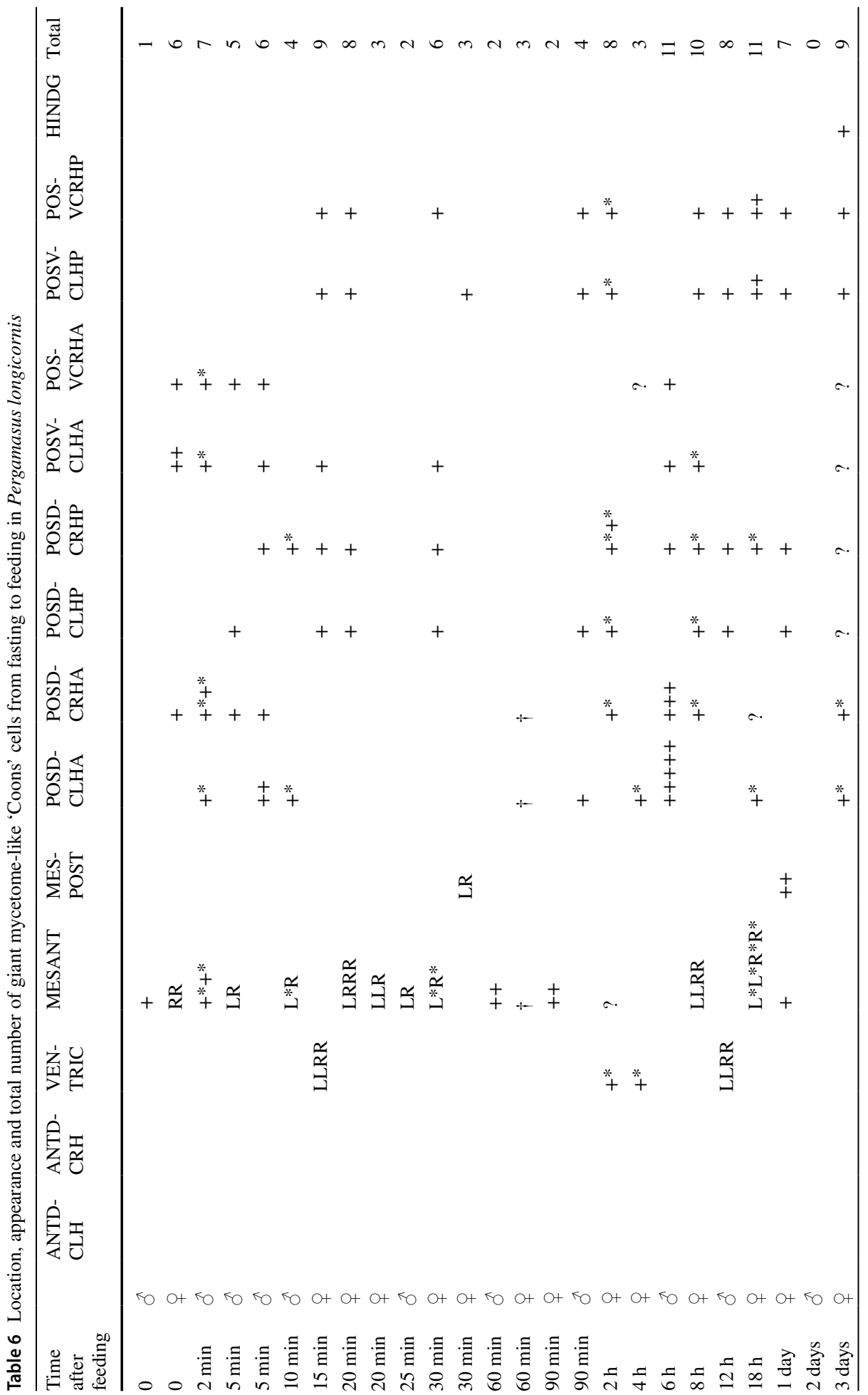




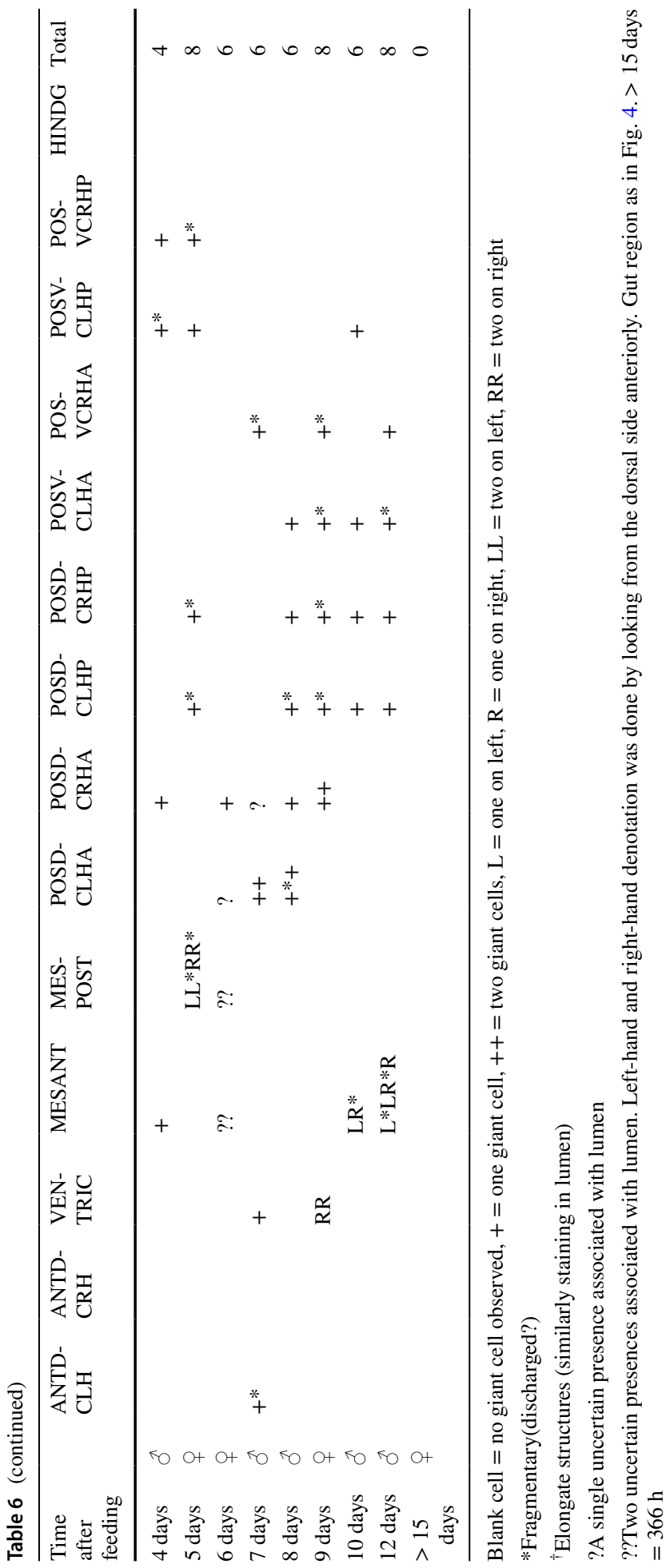

\section{Springer}


and almost terminal to posterodorsal caeca) matches that of the non-yeast mycetomes illustrated by Piekarski (1935) (see his Abb.7) in Ophionyssus natricis. Piekarski (1935) discusses these symbionts in great detail. Young (1968a) describes mycetomes in Haemogamasus ambulans in five similar places too-the ventriculus, the caudal tips of the two (postero)ventral caeca, and the mid-dorsal regions of the two posterodorsal caeca and the central posterodorsal caecum (i.e., the posterior mesenteron). Their relative size to the gut epithelium in $P$. longicornis was similar to the relative proportions of mycetomes illustrated in L. saurarum by Reichenow (1922) (see his Fig. 1) - and their position is similar in L. saurarum. Their location, size and appearance closely match the two pairs of globulose 'dark staining bodies' cells or glands described by Jakeman (1961) in Echinolaelaps echidnius (see his Fig. 45). They resemble the 'zooglea' described by Warren (1947) in mites.

Exactly what they are is not clear. They are probably not flagellates (Kreier 1977a). Rather, there are many parasitic protozoa known in arthropods (Kreier 1977b). In P. longicornis, these 'giant cells' apparently contain large numbers of red Mallory stain positive 'spores' of shape each like a vertebrate erythrocyte 'edge-on' and of substantial size in comparison to a typical gut cell nucleus. Confusingly, they resemble the "Secretzellen" that Roesler (1934) describes in being limited to the anterior part of the ixodid stomach (see $d$ and e in his Fig. 1). At various times they appear to be partially disintegrating and associated with the lumen in P. longicornis. At other times they were almost external to the gut epithelium embedded on the haemocoelic side. An individual $P$. longicornis could have evidence of 'infection' of the same host with 'Coons' cells in both the mesenteron and posteriorly in more than one caecal terminus at the same time. Coons (1978) states that the cellular inclusions in macrochelid giant cells decrease greatly in number on starvation for $72 \mathrm{~h}$. For sure, starvation can affect the levels of bacterial infection in phytoseiids (Wu and Hoy 2012). However, few changes with digestive state were observed in pergamasid 'Coons' cells which were commonly present—but intermittently at any one location. They did not radically change in the 34 mites studied (Table 6) unlike the gentle decrease after 72 h starvation in M. muscaedomesticae-Coons (1978). Hughes (1952) similarly recounts no changes in appearance of the dermanyssid mycetome during the digestive process. Woodring and Cook (1962) describes a few enlarged cells of the mesenteron with no nuclei, packed with greenish hyaline granules shaped like mammalian red blood cells in the oribatid Ceratozetes cisalpinus. Could P. longicornis be eating such from their crytpostigmatid prey and becoming similarly 'infested'?

The giant cells may be harmful to the pergamasid-Davidson (1980) summarises known pathogens of mites. Sponge-like giant bodies are known in insects (Huger and Krieg 1967) too. Relatively large mycetome cells, sometimes gigantic, change and divide with the life cycle of the insect host and become colonised by and release symbiotic microorganisms (Richards and Brooks 1958). In the pergamasid, these giant cells did not look like a generalised acarine phycomycete infestation such as Entomophthora (Weiser and Daniel 1969; Weiser 1972; Kal'vish et al. 1978). The individual 'spores' appeared larger and more numerously packed-in than the typical Wolbachia endosymbiont found infesting arthropods (Schütte and Dicke 2008) and often found in their reproductive systems (Burgdorfer et al. 1973). Pergamasids do not feed on blood so they are unlikely to be spirochetes like Borrelia etc as in tick guts (Sidorov 1960a, b). They are too big to be most rickettsias or bacteria known in ticks-Roshdy 1961b; Hecker et al. 1968; Friedhoff 1970; Čiampor and Nosek 1976 [although some acarine intracellular rickettsias in gastric caeca are up to $4 \mu \mathrm{m}$ long-Wright et al. (1984)]. They were not like Spiroplasma in phytoseiids (Enigl and Schausberger 2007) nor were they exactly like the small intracellular Giemsa positive micro-organismic bodies in the gut, ovaries and eggs of Dermacentor variabilis 
(Trager 1939). Although their location in the tips of the (anterolateral) diverticula is similar to them. The contents of the giant cells did not look like microfilariae or nematodes found in the gut and haemocoel of Liponyssus bacoti described by Hughes (1950b) either. Whilst generally similar to, they were much smaller than Babesia merozites found in ticks (Potgieter et al. 1976).

Sporozoans have been found in the haemocoel and also inside a large cell in the astigmatid T. dimidiatus (cf. T.longior) by Oboussier (1939). They are also known from the myrmecophilous mesostigmatid Urodinychus sp. (Warren 1944). However, these trophozoite gregarines (Paramonocystis?) show a more solitary occurrence and are too big compared to those in pergamasids. Gregarinids are known in acarid mites-Kostenko et al. (1978) and other arachnids-Devdhar and Amoji (1978). The pergamasid giant cells are rather packed out just like the spore containing sporoblasts of Urodinychus mid-gut or the infection of their Malpighian tubule cells with the haplosporidian Acoccidium tubulorum (Warren 1944-his Fig. 6). Microsporidian parasites are known in soil oribatids (Purrini and Weiser 1981). Moreover, a haplosporidian is known in Hermannia sp. (Warren 1944) whose location of infection matches the main location of giant cells in P. longicornis. Furthermore, the spore forming sporonts in uropodids (Warren 1944-his Fig. 7) look very much like smaller versions of 'Coons cells' with slightly smaller numbers of sporozoites or spores per sporoblast. Could the pergamasid giant cells be cross-infections? Could they be Bertramia or Coccidium spp.? Mature protozoan pathogen microsporidia spores do not stain with PAS, rather they stain positive to acid fuchsin (the red ingredient of Mallory stain) and are gram positive. It is tempting to conclude therefore that 'Coons cells' are these. However, microsporidia spores are usually oval 1 by $4 \mu \mathrm{m}$ and often they are not localised in one tissue or cell type but throughout the body of a mite (and if infected, it is a severe infection usually). Three different types of them have been found, transmitted vertically via the egg to both males and females and usually none in prey mites. The parasite seems to initiate infection in the nuclei of digestive cells. The digestive cells are sloughed off into caecal atrium where they cause great deal of damage to the caecal wall (Susan Bjornson pers. comm.). If 'Coons' cells are sporozoans, could this cellular damage be the origin of the unexplained lumenal material scored as 'Other' in Bowman (2017b) in the individual mite examined at $2 \mathrm{~h}$ post start of feeding?

The giant cell in P. longicornis might be a bacteriocyte-possibly like Cardinium or Solitalea found in acarids. However, they are not exactly like the Bacteroidetes individuals described by Hoy and Jeyaprakash (2008). Each 'corpuscle' in P. longicornis appears bigger than the Buchnera aphidicola symbionts found in pea aphids for instance. They appear more like large variants of the yeast-like organisms found in trombiculids (Kroman et al. 1969; Kroman 1979). They resemble the syncytial yeast-like symbionts of Scaphoideus titanus (Hemiptera: Cicadellidae)_Sacchi et al. (2008)—more than resembling Cardinium symbionts. They are not like the micro-organisms found in the Malpighian tubules of Argas persicus (Jaschke 1933) nor the cocci in the haemocytes, eggs and Malpighian tubules of other ticks (Mudrow 1933). Rather, in shape individually the contents of these pergamasid giant cells, resemble more the symbionts a-f, j, o-q in Fig. 3 of Reichenow (1922). Their packed nature also matches those illustrated in L. saurum in Reichenow (1922)'s Figs. 2 and 4 (but not the leech mycetome-his Fig. 8). The elongate similarly staining structures seen $1 \mathrm{~h}$ after the start of feeding (Table $6 \dagger$ ) may be those forms of mesostigmatid symbionts illustrated by Reichenow (1922) as k and 1 . Their association with the lumen at this time as a time of 'release' might be linked to the state of the lumenal material scored as 'Other' in Bowman (2017b) at $2 \mathrm{~h}$ post start of feeding. 
Whatever they are (sporidians, bacteriocytes or yeast-like symbionts), further detailed work to track 'Giant mycetome-like cells' in other pergamasids over time is needed.

\section{Discussion}

Each of the gut epithelial types (i.e., squamous, columnar, transitional pseudo-stratified) and their three major cell types (thin, square, jigsaw-piece) observed are considered below in turn together with the histochemical and quantitative evidence for the processes that may be going on in P. longicornis. The fact that the kinetic fits required separate processes supports this view of taking each cell type separately and then considering first its increase and then considering its decrease as distinct when deconvoluting the physiological phenomena. Vacuolation and other cells types will be then considered [below, including testing the specific 'Checks (a)-(f)'] in an integrated way and a final functional synthesis is attempted.

\section{Simple squamous epithelium formed of 'Thin' cells}

The cells described as 'thin' in this study of $P$. longicornis typify one of several elements of the mites' starved state (at time $=0$ ) - especially so in the posterior mid-gut. They are like those at 1 min post feeding in both $P$. persimilis described by Akimov and Starovir (1974), and in A. andersoni described by Akimov and Starovir (1977) (especially their Fig. b lower) - in being 'low and broad' with their height much less than their width. They rapidly increase in overall frequency $10 \mathrm{~min}$ post commencement of feeding in P. longicornis and are present in all regions up to about $2 \mathrm{~h}$ (see Fig. 4) when essentially they abruptly vanish. An exponential process poorly estimates this disappearance. Histologically, they appear to match: those found in the 'Magen' (stomach) of Tyrophagus dimidiatus (cf. T. longior) denoted by Oboussier (1939) as "ziemlich grossen, flachen Zellen"; those illustrated by Grandjean and Aeschlimann (1973) as forming the epithelium of a newly engorged female argasid tick Ornithodoros moubata; and, the groups of flat cells present in the mid-gut epithelium of Myobia murismusculi (Filimonova 2001). They match those found in the female ixodid early feeding stage that Roesler (1934) describes as "Schleißlich wird die Darmwand derartig staff, daß alle Zellen als ganz schmaler Saum um das zehnund mehrfache gedehnt das Lumen umspannen." (see his Fig. 4).

In $P$. longicornis, a gut lumen is present within $5 \mathrm{~min}$ of starting feeding (Bowman 2017a) and established consistently when the gut is first initially filled with watery prey material by $10 \mathrm{~min}$ (Bowman 2014). The thin cells surround this gut lumen forming a flat undifferentiated simple squamous epithelium. Granular imbibed prey material is the predominant material in this lumen (by 15-20 min from the start of feeding-Bowman 2017a). Thin cells are probably not the 'reduced digestive phase cells' found in ticks (Alberti and Coons 1999). Rather, physical changes of pergamasid lumen content would be expected to force and stretch existing mid-gut epithelial cells flattening them-presumably until the end of imbibition (around 56-96 min) stopping this process. So, these thin cells are taken simply to be the 'basal' state for the epithelial cells in the majority of the pergamasid gut when it is maximally stretched by the imbibition of fluidised prey tissue. Other authors do not so often mention this cell type. This would be expected for studies using phytoseiid predators or any other mesostigmatid which has to consume several eggs or say small tetranychid prey before being satiated — each mini-bolus of food material being imbibed being insufficient in its own right to markedly expand the mid-gut and so strongly 
flatten the epithelium. In this pergamasid study intentionally a bolus of prey approximating or exceeding the body volume of the edaphic predator was used to exaggerate changes. As such these thin cells may represent the (non-flattened) undifferentiated 'true gut' cells of Roesler 1934; Neumann 1941; and, the Soviet (at the time) researchers like Akimov and Starovir. However, how to test this conjecture?

If the occurrence of thin cells was in fact driven by gut expansion through the ingestion of material (whether externally triturated granular material sucked in or clear fluid prey material being drunk, which itself drives the appearance of a lumen and expands the cells from the resting starved state), then before any cell transformation or egestion commences to winnow them away, one might expect the rate of thin cell appearance to broadly match the rate of the appearance of a gut lumen. In fact, lumenal kinetics show multiple phases (Bowman 2017b). There is an almost instant appearance of a lumen with a very short halflife and consistent establishment by $10 \mathrm{~min}$, followed by two phases of more concerted growth showing half-lives of $33 \mathrm{~min}$ to $2.7 \mathrm{~h}$. The mid phase is worth considering. The first major rise in thin cell occurrence is at $10 \mathrm{~min}$ too-supporting a possible congruent physical cause by drinking. The related mesostigmatid Haemolaelaps casalis is known to drink fluids (McKinley 1963). Furthermore, Table 1 shows overall steady growth of thin cells with a half life of 31.8 min-exactly in the right ball-park to also support the idea that they are produced in the mid phase of lumenal expansion. Splitting this thin cell appearance into a slow and fast phase to match Bowman (2017b) shows little change to the overall speed of response and is not strongly supported statistically (results not shown). Production by physical expansion and stretching is however supported by the kinetics of the fast phase of appearance of thin cells (22.9 $\mathrm{min}$ ) nicely matching the input kinetics of the lumenal granular material (Bowman 2017b). Three to five half-lives (Table 1) matches the 56-96 min feeding period on prey. Also the period of this fast phase of noticeable increase in thin cells (see Fig. 7) matches the time of feeding when coxal fluid recycling is postulated. The gut is being kept expanded by continual re-imbibition of coxal fluid 'washing-in' the extra-corporeally digested prey tissues like applying saliva to food (Bowman 2014). Alberti (1979) suggested that solifugid coxal fluid may act as saliva in the same way. Free-living predators on small infrequent prey must have efficient prey-fluid conservation compared to obligate ecto- and endo-parasites or solid feeding mesostigmatids (Wernz and Krantz 1976). Note that ammonia could be vapourizing off this excreted fluid-if pergamasids are shown to be ammoniotelic like isopods (Hoese 1981). The faster kinetics of the appearance of the lumen occurrence compared to the thin cells appearing would make sense as any scoring of a lumen presence is almost instantaneous of it appearing-well before starved cells in a transitional pseudo-stratified epithelium are flattened out by significant lumenal expansion on prey drinking and scored as 'thin'. The conjecture is supported.

Figure 4 shows the zenith of the occurrence of thin cells around the switch from ingestion to digestion predominating [at $2 \mathrm{~h}-$ Bowman (2014)]. They vacuolate strongly around the end of feeding (56-96 min-Bowman 1987a) suggesting either food take-up from the lumen or secretory production into it (Fig. 7). Their similar vacuolation at this time to that of the square cells and the complete disappearance of thin cells during the digestion predominating phase ( $>2 \mathrm{~h}$ after feeding commences) suggests that they are probably the 'younger' stage of the cells denoted as 'square'. However, the lack of vacuolation in thin cells until about 30 min after the start of feeding (Fig. 7), suggests that before this point (i.e., in the slow 'appearing' phase), the squamous epithelium may be active transporting fluids (through a histologically unseen mechanism or mechanisms-Ahearn and Hadley 1977) rather than intracellularly taking up food or secreting enzymes. This lack of vacuolation does match the early first part of the period when coxal droplets are seen. Note 
that granular imbibed prey material arrives in the pergamasid lumen of the gut just before vacuolation is regularly seen (Fig. 7) - occurring in the second part of the coxal droplet formation phase. It is tempting to conclude that the vacuolated thin cells here are taking up ingested granular material (and sensibly at a rate clearly faster than its arrival in the lumen).

The squamous epithelium is not universally present over all times in the mid-gut of $P$. longicornis. Where do the thin cells go? Thin cells are completely missing by $2 \mathrm{~h}$ - the breakpoint between ingestion dominating and digestion predominating (Bowman 2014). They become absent soon after actual feeding stops, catastrophically declining away with a half-life of $49.5 \mathrm{~min}$ - slightly slower than their appearance (i.e., they exhibit 'flip-flop' kinetics approximately). This is the time that an increase in square (digestive) cells gets under way once feeding stops (see Fig. 14) suggesting that the thin cells grow to become them as the epithelium changes its form. Does examination of square cell kinetics support this ageing conjecture?

\section{Simple cuboidal or simple columnar epithelium formed of 'Square' cells}

Figure 5 shows that modest numbers of square cells appear to also be a sporadic resting or ground-state for the gut epithelium at time $=0$, i.e., when fasted. These are present amongst the thin and irregularly shaped jigsaw-piece like tessellated cells on the histological sections at this time. Their occurrence increases over time after the start of feeding much like the pro-digest cells of Agyei and Runham (1995) in ticks. They appear to be like the epithelial cells of P. persimilis (Akimov and Starovir 1974) at $15 \mathrm{~min}$ and those of $A$. andersoni (Akimov and Starovir 1977) at $10 \mathrm{~min}$ after the start of feeding. In that sense they could be considered as the 'younger digestive cells' described by Starovir (1982). Their increase in height matches the description of gut wall changes by Neumann (1941) in Parasitus.

The kinetics of square cell appearance (Table 1) matches that of the thin cells and the 2 $\mathrm{h}$ switch from ingestion predominating to digestion predominating - they achieve maximal presence around $2-3.3 \mathrm{~h}$ post start of feeding. This consilience suggests a common process for the appearance of these two types of gut epithelial cells. However, square cells do not disappear abruptly. Square cell kinetics and their predominance broadly matches the appearance and presence of granular and globular lumenal prey material (but not membraneous or refractive grains Bowman 2017b) in the gut lumen up until about $4 \mathrm{~h}$ after the start of feeding (see Fig. 8). So they appear to first match the 'initial digestive cells', then the 'detached digestive cells' function in Agyei and Runham (1995)'s scheme of tick guts.

The occurrence of square cells, despite prey material vanishing, persists during the rise of refractive grains in the lumen (Bowman 2017b), not falling appreciably (with a slow half life) until the production of membraneous faecal material (7-8 days after the start of feeding) and the onset of fasting/starvation around 10 days (Bowman 2017a) after the start of feeding. Their disappearance and change in gut epithelial form is slow- 3 to 5 half lives are of the order of 2-3 weeks congruent with the time to completely clear the idiosoma of excretory material (15 days-Bowman 2017a). This suggests it may be possible for $P$. longicornis to successfully starve for nearly a month after a single large meal in the wild - an undoubted advantage for a long-lived predator faced with the challenge of rare periodic feeding in the soil litter environment. The related predatory mites Macrocheles subbadius, Cheiroseius (= Sejus) necorniger and Ololaelaps venetus are able to sustain starvation in the laboratory for: more than 3 months; about one month; and, about two 

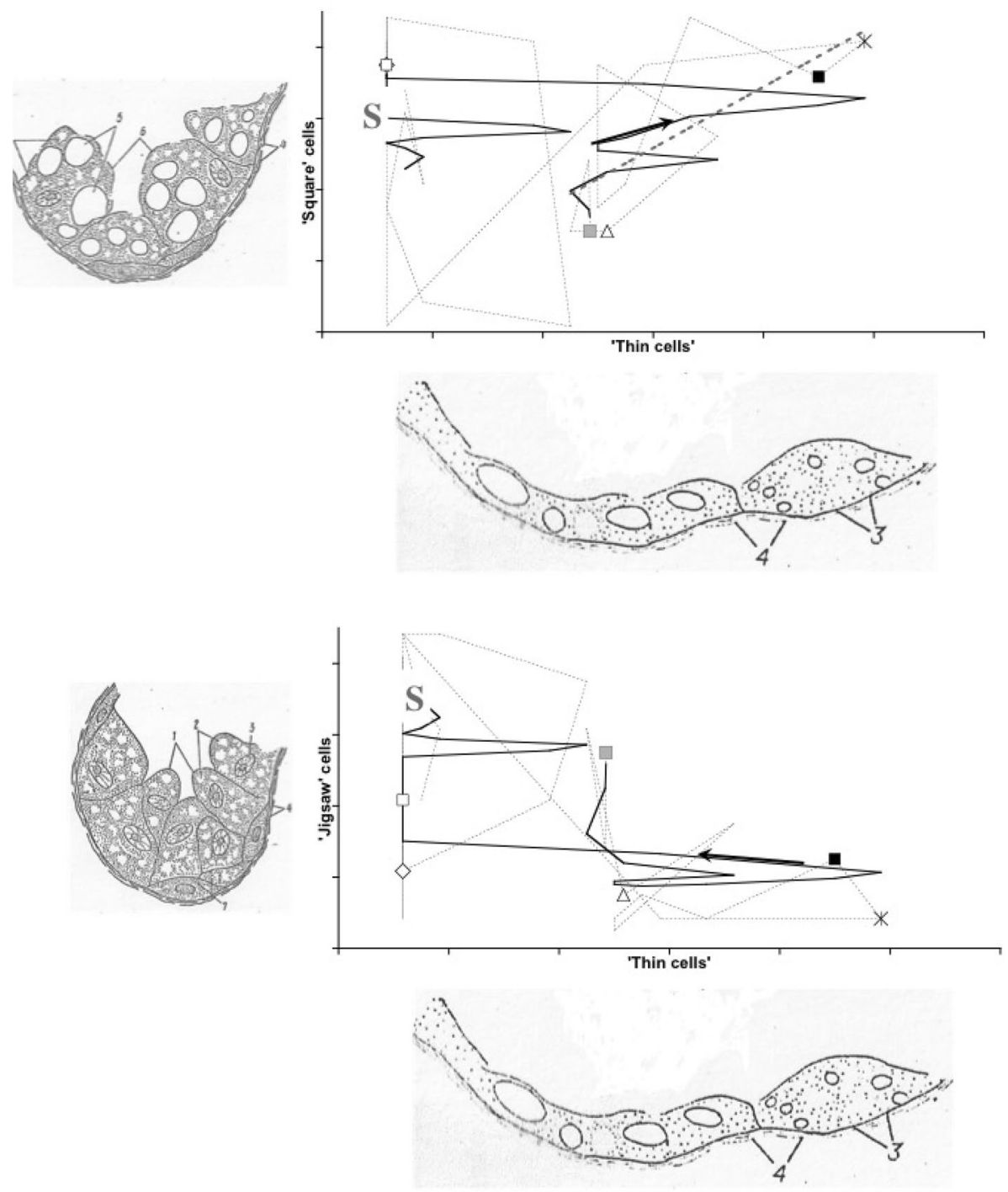

Fig. 14 Relationship of avacuolate thin cells to other avacuolate cells from feeding to fasting in Pergamasus longicornis-showing rippling hysteresis (average data as grey dotted line with jointly smoothed trend in black dashes-black arrow indicates time increasing) between simple squamous epithelium and either pseudo-stratified (upper) or cuboidal (lower) epithelium. Note grey square is start of feeding. Asterisk indicates the 120 min optimal knot position between net gut-filling and net gut-emptying (Bowman 2014). Black square indicates approximate mean feeding time (Bowman 1987a). Grey S indicates approximate time starvation commences-Bowman (2014). Open symbols as in Fig. 7. Square and thin cell prevalence across the gut rises during imbibition phase (heavy dashed grey line is linear trend up to the end of feeding). Then thin cell types vanish by $4 \mathrm{~h}$ after the start of feeding presumably by interconversion to square and jigsaw cells. Upper: avacuolate thin cell score across gut regions to avacuolate square cell score. Lower: avacuolate thin cell score across gut regions to avacuolate jigsaw cell score. Illustrative section showing thin cells (here 1 min after feeding with a few vacuoles in Amblyseius andersoni from Akimov and Starovir (1977)_CVestnik Zoologii with permission. $3=$ basal membrane, $4=$ muscle fibres. Section of square cells (fed state) and Jigsaw cells (unfed state) in Amblyseius herbarius from Starovir (1979a)—CVestnik Zoologii with permission. 1 = 'secretory cells', 2 = 'vacuoles' (here non-food pale areas), 3 = nuclei with nucleoli, $4=$ basement membrane, $5=$ food inclusions (true vacuoles), $6=$ 'digestive cells', $7=$ 'undifferentiated cells' 
months, respectively (Luxton 1966) suggesting a class-level fasting competency in mesostigmatids matching that of pseudoscorpions (Jones 1975). Haemolaelaps casalis can last 2 months without food (McKinley 1963). Even the small haematophagous rat mite Echinolaelaps echidninus can survive at least 24 days starvation in good environmental conditions (Knülle 1967, but only 7-8 days without water according to Owen in McKinley 1963). Predaceous phytoseiid species can live hungry for 3.7-17.7 days (Kolodochoka and Lysaya 1976). Given water and cool conditions (i.e., $22^{\circ} \mathrm{C}$ ), amblyseiids can survive without food more than 27 days (Blommers et al. 1977) and B. bacoti can survive 160-141 days (Belozerov 1957). This contrasts with the rapid utilisation of reserves in and fast death in obligate crypto-parasite Varroa jacobsoni (Barabanova 1991) and the short survival times without food for the phoretic parasitid P. necrophori (Belozerov 1957). This starvation resistance capacity would be an useful adaptation for exposed unpredictable environments in P. longicornis.

What are these square cells comparatively? They may be the smooth digestive cells described by Akimov and Starovir in their multiple Soviet (at the time) publications on phytoseiids. The 'square' cells in the pergamasid gut epithelium appear to match the "annähernd gleichgrossen polygonalen... ..Zellen" described in acarid stomach and caecal wall epithelia by Prasse (1967). In that paper, the latter cells are laid out as the basis of two types of cells: "Verdauungszelle" (digestive cell); and, "Drusenzelle" (secretory cell). Each of these enlarge, vacuolate and become clavate during acarid digestion. Some may break off or dissolve into the lumen-the two types being mainly defined by the staining properties of their cytoplasmic inclusions. However, using the Mallory stain colour changes were consistent. No distinction was found amongst the square cells in P. longicornis - all appeared to be both or either identical 'digestive cells' or 'secretory cells'.

The square cells might match the 'empty-looking columnar or cuboidal cells' of Argas persicus - Tatchell (1964). These cells in ticks with saliva-fast PAS positive granules disintegrate into a colloidal gut-acting lysate in advance of vacuolation during food uptake into the cells. If present in P. longicornis these could be the cause of globular material appearing in the lumen (Bowman 2017b). What of enzymes? For sure, increased protease activity immediately after feeding in argasids indicates the presence of an inert, readily activated precursor molecule (Tatchell et al. 1972). Bowman (1984a) describes lumenal border associated acid phosphatase in gut epithelial cells during digestion-an enzyme known in the absorbing cells of the duodenal villi of rodents (Hugon and Borgers 1968) and involved in Hyalomma asiaticum heterophagosomes (Raikhel 1975). This enzyme rapidly increases in the salivary glands of the tick $R$. appendiculatus as feeding commences (McCall 1976) and is localised strongly in the gut epithelium of other invertebrates (Barry and Mawdesley-Thomas 1968). Acid phosphatase positive lysosomal vesicles indicative of the same intracellular digestion as in mammals have been described in the tick B. microplus (Gough and Kemp 1995). In contrast, alkali phosphatase is concentrated amongst the transporting membranes in argasids (Tatchell 1964) and the apical membrane regions of the mid-gut epithelial cells involved in the absorption and intracellular digestion of haemoglobin of ixodids (Agyei et al. 1992). Alkali phosphatase usually marks out brush borders and other transport active cell membranes (Boyer 1971; Welsh and Storch 1976). Acid and alkaline phosphatase (along with phosphodiesterase) are known from Tetranychus urticae (Kötter 1978; Humiczewska and Mielnicka 1983). Baker (1977) describes vacuolar non-specific esterase, acid and alkaline phosphatase activity in the molluscan parasitic mite Unionicola intermedia. Esterases are known in spider mites-McEnroe (1936a). Esterases breakdown esters in food and were found abundant by Tatchell (1964) in the basal areas of intracellularly digesting argasid cells. Considering the pergamasid enzyme histochemical results 
(Tables 2, 3, 4, 5) it is therefore tempting to conclude that the cuboidal epithelium of square gut cells in P. longicornis is at least both transporting and assimilating prey food as well as growing. Further detailed enzyme cytochemistry is needed to test this conjecture.

Vacuolated square cells (like those in Fig. 1a of Roesler 1934, or Fig. b of Starovir 1979a) are broadly correlated with avacuolated square cells $\left(r^{2}=0.66\right)$ in P. longicornis. The lack of vacuolation in square cells until about 30 min after the start of feeding (see Fig. 8) suggests that before this, the gut epithelium may be actively transporting fluids rather than intracellularly taking up food or secreting enzymes. This would match the early part of the period when coxal droplets are seen. Finding cellular alkaline phosphatase histochemically at more various early time points would be a key confirmatory evidence in future studies as this enzyme is common cytologically in fluid transporting tissues (such as in arachnid coxal glands-Gabe 1968). Vacuolation in this cell type occurs in the second part of the coxal droplet formation phase. Thereafter the repeated cycles of vacuolation (and de-vacuolation) seen through to the worst-case total feeding cycle time are illusions based upon the destructive sampling of different mites. Overall vacuolation declines (as do the presence of square cells) to the start of starvation around 9 days post commencement of feeding. It is assumed that starvation would drive metabolic changes like in the plant prostigmatid $T$. urticae where there is an alteration in intermediate metabolism after starvation (Kötter 1978) with a strong increase in glucose content and drastic reduction in stored glycogen content. Glycohistochemistry (Raikhel 1974a) could confirm this in pergamasids. Monitoring concomitant levels of carbohydrases (already known in tetranychids-Erhardt and Voss 1961) could be useful too.

Figure 8 shows how firstly square cells with small vacuoles appear, quickly followed by those with large vacuoles within $10 \mathrm{~min}$ of the start of feeding. A columnar epithelium comprised of square cells with large vacuoles then predominates throughout the gut until about $18 \mathrm{~h}$ after the start of feeding when it declines-square cells with small vacuoles once again coming to the fore. Only by 4 days after the start of feeding do avacuolate square cells predominate again. This set of transitions after the best estimate of initial gut emptying time of $12.5 \mathrm{~h}$ is coincident with the appearance and rise in egestive (and faecal) material in the gut lumen. The kinetics of square cell disappearance (Table 1) nicely matches the slow elimination phase of both granular and refractive grain material in the lumen (Bowman 2017b) suggesting that square cells are (one of) the main rate limiting engines in prey digestion. This gut epithelial 'engine', early-on predominately taking up and digesting food, then late-on predominately catabolising and egesting absorbed prey material is supported. This also matches the period of stasis in gut size described by Bowman (2014). There is no clear evidence in this study that square cells are transformed to any other type of cell over time other than by shrinkage (Fig. 15). Further work should determine what proteolytic machinery these cells might contribute to any dynamic multienzyme network capable of diverse food processing as in ticks (Franta et al. 2010; Sojka et al. 2013).

Avacuolate, small and large vacuole-containing cells show broadly similar patterns over time. Resting fasted avacuolate square cells develop first small vacuoles then large ones within $10 \mathrm{~min}$ of the start of feeding - just like the increase in intracellular nutrient granules over time in A. andersoni (Akimov and Starovir 1977). All square cell types persist thereafter until cells with large vacuoles decline in number, allowing those with small vacuoles to predominate from about $18 \mathrm{~h}$ after the start of feeding. The latter pergamasid cells may thus be like the degenerative cells described by Tatchell (1964) in argasid guts which are filled with residual material basally but still possess small food vacuoles apically. This preponderance of cuboidal cells with small 'nutrient granules' late on (3-9 h after feeding) 


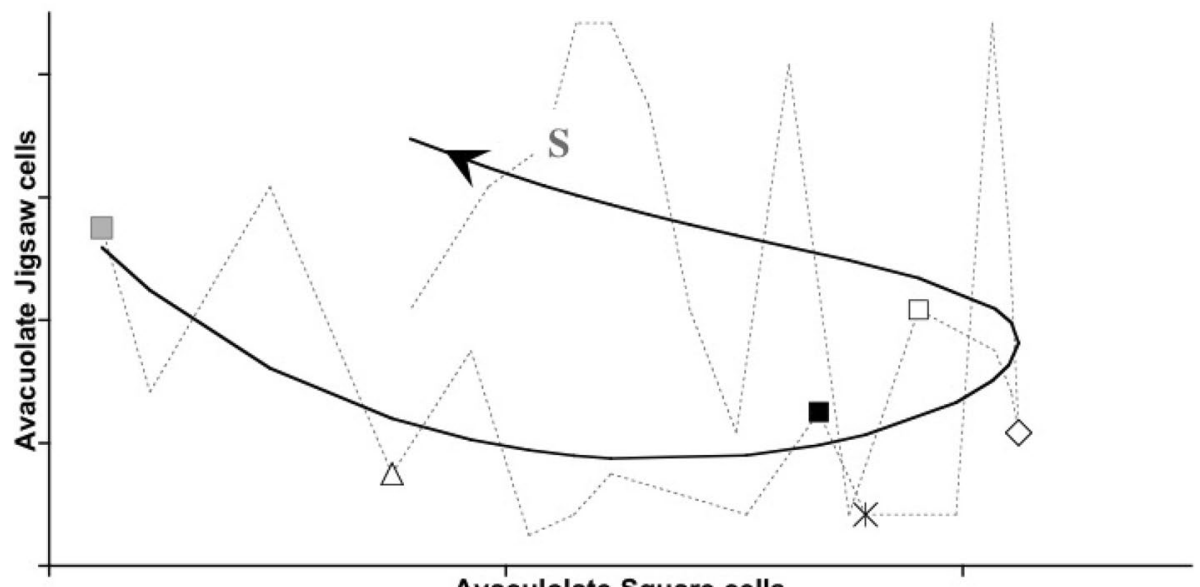

Avaculolate Square cells
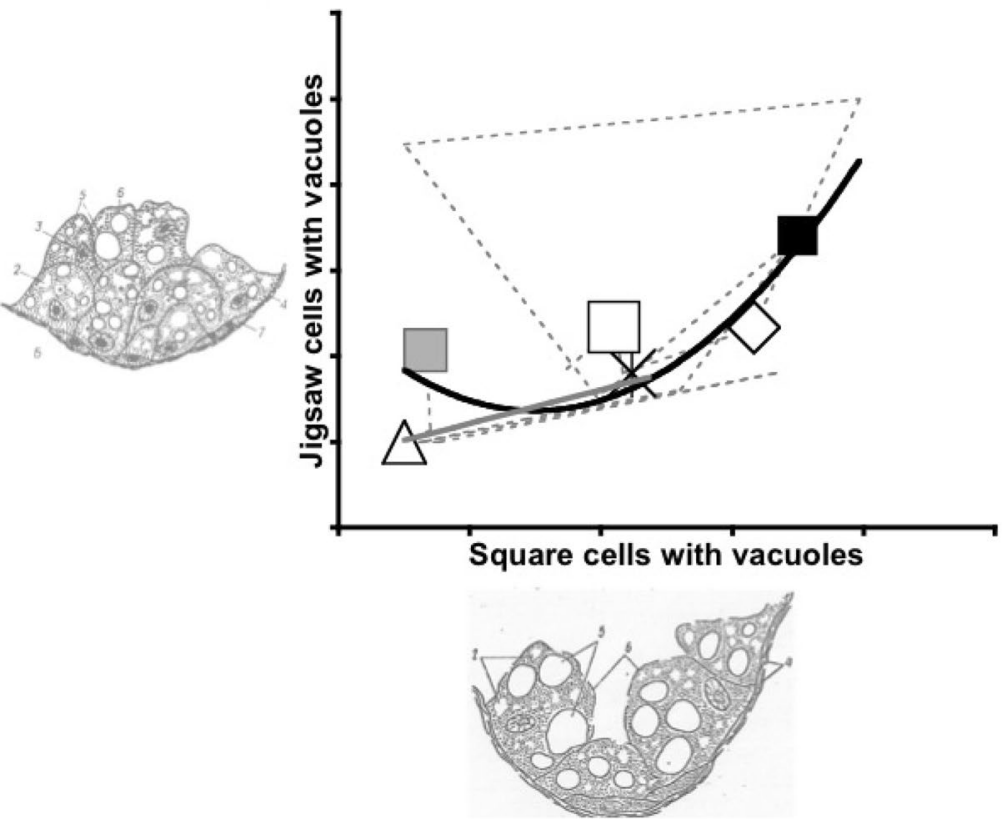

Fig. 15 Interrelationship of square and jigsaw-piece cells from feeding to fasting in Pergamasus longicornis. Upper: rippling hysteresis between avacuolate 'jigsaw' cells and avacuolate 'square' cells (grey dotted line). Average data as grey dotted line with jointly smoothed trend (black arrow indicates time increasing). Lower: vacuolated jigsaw and square cells vary together during digestive cycle (no hysteresis)—solid line smoothed data, grey solid line linear trend up to point of change from net gut-filling to net-gut emptying. Grey square is start of feeding. Asterisk indicates the $120 \mathrm{~min}$ optimal knot position between net gut-filling and net gut-emptying (Bowman 2014). Black square indicates approximate mean feeding time (Bowman 1987a). Grey S indicates approximate time starvation commences-Bowman (2014). Open symbols as in Fig. 7. Square cell section from Amblyseius spp. with permission as in Fig. 14. Section showing Jigsaw cells (fed state) in Androlaelaps casalis from Starovir (1982)_- OVestnik Zoologii with permission. 2 $=$ 'vacuoles' (here non-food pale areas), $3=$ nuclei with nucleoli, $4=$ basement membrane, $5=$ food inclusions (true vacuoles), $6=$ 'digestive cells', $7=$ 'undifferentiated cells' 
was also found by Akimov and Starovir (1977) in A. andersoni. In that mite, 'nutrient granules' were partly undergoing breakdown with the formation of crystals. Degenerative digestive cells do occur with undigested food residues in tetranychids (Akimov and Dei 1973).

Square cells with small vacuoles begin to decline around 4 days after the start of feeding being replaced by avacuolate cells. The cuboidal epithelium of square cells broadly follows the pattern of lumenal presence after the period of initial gut filling-persisting until the end of the feeding and digestive cycle. The early rise matches the lumenal score for prey material (marbled line to the left of the break marked at $\$$ being [granular + globular + other contents] in Fig. 8-Bowman 2017b) up to $6 \mathrm{~h}$ after the start of feeding. Thereafter the square cells match the pattern of the rise of [refractive grains+membraneous faecal material] in the lumen (marbled line to the right of the break at \$ in Fig. 8-(Bowman 2017b), not falling appreciably until the onset of fasting/starvation around 9 days after start of feeding (Bowman 2017a). In this phase, the kinetics of square cell disappearance (Table 1) broadly agrees with the kinetics of the disappearance of granular material from the lumen (Bowman 2017b) confirming the columnar epithelium of square cells being the main primary digestive engine in P. longicornis.

These cells probably switch into accumulating various residual bodies (and perhaps spherites?) - being transformed into degenerative egestive cells (as in S. fringilla-Filimonova 2009). Having an egestive mechanism involved in mineral containing spherite production could be an advantage for P. longicornis as they feed on oribatids (Luxton 1966) which are implicated in mineral cycling (Carter and Cragg 1977) and have 10 times the calcium content and twice the potassium content of mesostigmatids (Wallwork 1983). These cations will need to be cleared effectively from the pergamasid idiosoma after ingestion undoubtedly along with faeces-especially if the prey is fungal specialising microphytophagous or panphytophagous mites (as fungi differentially concentrate calciumCromack et al. 1977). This with its contingent ion-balance issue is the same challenge as that faced by solifugids (Klann 2009).

Whether square cells with a degenerative egestive role are extruded wholly into the lumen (i.e., holocrinally as in some oribatids-Tarman 1968) is probably unlikely given that free cells are seen late on in P. longicornis. Whether square cells with an egestive role lose solely their apical content into the lumen eccrinally (i.e., merocrinally) or apocrinally as in other oribatids (Tarman 1968) to then fall-back to being jigsaw-piece cells is not clear. Grandjean and Aeschlimann (1973) recount holo-, mero- and apocrinal gut cell disintegration in argasids. Šustr and Hubert (1999) notes apocrine secretion in the mesenteral and caecal cells of Galumna elimata. Both apocrine and merocrine mechanisms are known in the hepatopancreas of other chelicerates-holocrine mechanisms being found in crustaceans (Herman and Preus 1972). Further TEM work is needed especially to see whether any altered mitochondria are associated with this excreting activity (Coons and Axtell 1971a).

\section{Stratified or transitional pseudo-stratified epithelium formed of a tessellation of 'Jigsaw-piece' like irregularly shaped cells}

This squamous/cuboidal/columnar tissue type matches: the multi-cell-layered wall of the uropodine ventriculus described by Ainscough (1960); the irregular shaped 'true gut cells' of P. kempersi (Neumann 1941); and, those of 'delicate cytoplasm' recorded by Roesler (1934). The unfed state, whether early on (time $=0$ ) or late on (e.g. 4 days onwards) is 
typified by these jigsaw-piece (i.e., irregularly shaped tessellated) cells in the tightly contracted mid-gut caeca forming a dense pseudo-stratified epithelium [all piled-up like in starved argasids-Tatchell (1964)]. Cellular boundaries sometimes can be hard to see. In uropodines this gut epidermis is several layers thick as indicated by the disposition of the nuclei with indistinct individual cell boundaries. A pseudo-stratified epithelium (similar throughout the mid-gut course) is the non-fed state in the lizard blood-sucking L. saurarum (Reichenow 1918). Non-vacuolated, jigsaw-like cells are shown in Fig. a of Starovir (1979a). Bi-laminar and multi-laminar gut epithelia characterise A.andersoni 12 and $24 \mathrm{~h}$ after feeding (note that in that mite $24 \mathrm{~h}$ equals one feeding cycle). This all points to at least some of this cell type comprising quiescent processes. Do they have small basal labyrinths typical of non-transporting epithelia? - a TEM follow-up study could check this.

Jigsaw-like cell predominance (ignoring any vacuolation) in P. longicornis is lower during the time of the presence of lumenal granular, globular and refractive grain material (see Fig. 9) up until the end of the feeding cycle. The suggestion is that these jigsaw cells during the latter active gut period are either stretched to become thin cells (see Fig. 14, lower) or fill out by the absorption of prey material into becoming square cells (see Fig. 14, upper). This conjecture is supported by the strong inverse correlation of jigsaw cell occurrence with the presence of a lumen (figure not shown). Insufficient variation in jigsaw cell occurrence prevents good kinetic parameter estimation. Jigsaw cells vacuolate in line with the vacuolation of square cells rising and falling in prevalence together during the digestive cycle and hence some jigsaw cells may be simply crushed variants of square cells (see Fig. 15, lower).

Within the pseudo-stratified epithelium, others of these jigsaw-like piece cells may represent the rough digestive cells described by earlier Soviet workers that degenerate attached to the basement membrane and release crystals (三 refractive grains herein?) Akimov and Starovir (1974)—or represent the secretory cells described by Akimov and Starovir (1977); Starovir (1979a). This can be nicely seen by the hysteresis between the avacuolated square and avacuolated jigsaw cell types (see Fig. 15, upper). There is a general inverse 'rippling' cyclical relationship with a lag between the two cell types, like two 'compartments' who alternately grow and then fall transferring from one to each other over time (waves of intracellular digestion/egestion?). This hysteresis is exaggerated by the epithelium of the anterior caeca being dominated by jigsaw-piece cells at first and the anterior mid-gut taking longer to fill during imbibition (Bowman 2014). Further support for their identity of function is that in both cell types, vacuolation slightly precedes their popularity (result not shown).

So perhaps late on in the feeding cycle, jigsaw-piece cells focus more on refractive grain release (eccrinally, apocrinally or even holocrinally) rather than focusing on food absorption and digestion? Whereas the cuboidal epithelium of square cells focuses more on prey material absorption and digestion and less on refractive grain release? Then, although the proportion of cells scored as square and jigsaw may change over time, the actual basis of each cell would be the same, with gut epidermal cells becoming just physically large (square) or physically shrunken (jigsaw) as the mite perhaps rhythmically moves towards completing the feeding cycle and final fasting/starvation-much as the data actually shows. TEM microscopy of jigsaw-piece cells might help confirm this conjecture of a role switch (as in S. fringilla-Filimonova 2009) if these cells are seen to be predominately structurally involved in egestive release. 


\section{Vacuolation, clavate and free cells}

No distinction of vacuoles as intracellular absorbed food depots versus secretions was made in the scoring in this histological study. Either origin is possible (Smrž and Čatská 1989). So, if there are special 'digestive' versus 'secretory' cells in P. longicornis they should both appear vacuolated micro-histologically but differ temporally in occurrence. Accordingly, the kinetics of vacuolation, the enzyme histochemical localisations and consideration of two more cell types ('free', 'clavate') will help inform how the proportions of each main gut cell type change over time and back-up or refute the above arguments on the main function of the gut epithelial tissue.

\section{Vacuolation}

Stainable and distinct (i.e., membrane limited) food vacuoles were observed plentiful in this histological study and are assumed to be analogous to the mesenteral and caecal cell granulation described by others [for instance Šustr and Hubert (1999)]. As in ticks (Till 1961) vacuoles ('globules') are at first small then increase in size as feeding progresses. Unlike in spiders (Ludwig and Alberti 1988b), no clear evidence of secretory granules being filled/stocked up in fasting animals was seen. As in Liponyssus (Reichenow 1918), all epithelial cells appear to have a liquid absorptive function. This is confirmed by the strong localisation of alkaline phosphatase in their lumenal margins during the early ingestion/osmoregulation phase (Table 4). Could these thin and early square cells at this time also have extensive basal labyrinths typical of epithelia involved in extensive transcellular transport (and not just only having characteristic apical differentiations)? A TEM follow-up study is needed to confirm if they are like the 'replacement cells' in the first continuous phase of ixodid digestion (Tarnowski and Coons 1989).

The significance of diffuse acidic phosphatase cell activity (Table 3) at this time is not clear. It could be a marker of a switch in general metabolic activity (i.e., rough endoplasmic reticulum/Golgi activity) as the thin cells are stretched out and switched on to become square cells-but that would mean that the lumenal activity observed is from imbibed prey material. Rather, it could represent lumenal acid phosphatase positive material being taken up by pinocytosis - this acid phosphatase initially arising from an extra-corporeal source (i.e., prey tissue + saliva). This latter idea is supported by: acid phosphatase being found in the salivary glands; esterase activity being found in the pre-oral cavity; esterase being found as traces throughout the lumen early on during imbibition; and, esterase activity being found in the salivary glands (Table 2). Esterases are already known in the saliva of cattle ticks (Geczy et al. 1971) and the digestive fluids of spiders (Mommsen 1978b). So this could be the case in $P$. longicornis. Alternatively a pulse of acidic phosphatase could be being formed in the rough endoplasmic reticulum/Golgi apparatus (Welsh and Storch 1976) and pinocytically secreted into the gut lumen from these active gut cells at this time to engender extracellular prey breakdown of easily imbibed compounds (e.g. to catabolise soluble phosphorylated dissolved compounds) inside the mite gut. Follow-up confirmatory work is needed.

There is a view (Jeffers and Roe 2008) that for both normal dietary and for xenobiotically derived proteins, such food material can be moved without modification of their primary or multimeric structure (i.e., retaining their functional characteristics) across the arthropod gut in a non-substrate specific way. This trans-cellular or intercellular pathway being via continuous diffusion and not by pinocytosis or the vesicular transport seen in 
mammalian systems. In this way accumulation in the haemolymph (and back in the gut lumen) would depend upon the balance of any extracellular separate protein degradation rate and the rate of transfer along this pathway. Of course, this would mean no vacuolation. Whether this occurs for any imbibed prey proteins in P. longicornis is not clear-immunohistochemical micro-investigation is needed to test this conjecture.

Stainable vacuoles were present in cells during the time that granular material is found in the lumen, peaking during the time of globular lumenal contents (inferring secretion activity?), then falling. As with granular prey food input (Bowman 2017b) there was no difference anteriorly to posteriorly across the mid- and hind-gut. Vacuolation was not correlated with the presence of a lumen-since a lumen persists during the phase of refractive granular material appearing lumenally just as the declining tail of vacuolation is observed.

Does the colour of vacuole staining (with Mallory's Triple Stain) give any insights? One must be cognoscente of the known caveats regarding the interpretability of histological stain colouring. All three dyes in Mallory's Triple Stain are acidic and bind to cationic compounds (i.e., not to nucleic acids, nor sulphate groups of glucosaminoglycans, nor the carboxyl groups of proteins). They bind to basic compounds and thus also to the amino groups of proteins. However, structural porosity is important, less porous tissues stain by the smallest molecular size dye, so if a larger dye can penetrate a fixed tissue it will displace the smaller molecule. This all can be affected by the local acidic pre-conditions deployed in the staining and cross-linking effects from fixation. So, absolute inference of chemical properties of the vacuoles from their colour is thus not straightforward. Fig. 16, upper shows the stacked raw observed probability of each colour staining for vacuoles over all vacuoles on the transverse sections for each time slice from feeding to fasting in $P$. longicornis (across all regions not including the rectal vesicle). This is effectively the conditional probability of vacuole colouring (i.e., $=p($ colour $\mid$ vacuole $)$ ). The rectal vesicle before 90 min and between 6 and 8 days inclusive showed occasional Mallory Triple Stain stained globules in its lumen (Fig. 16, middle). With Mallory Triple stain in mammals: elastic fibrils, red blood cells and nucleoli normally stain pink or yellow; cytoplasm and neuroglia stain red (i.e., with acid fuchsin); and, collagen fibrils, mucus and ground substance stain blue (i.e., with aniline blue). Eosinophilic granules stain orange (i.e., with Orange G). It is not clear what these behaviours might match to chemically in acarines. Allowing for the probability of there being a vacuole at all [i.e., p(vacuole)]—see Fig. 11), yields by multiplication the probability for colour staining in vacuole numbers present over the mid- and hind-gut (i.e., = p(colour \& vacuole $)$ ) as in Fig. 16, lower. Yellow vacuoles were only observed in the posterior epidermal regions of the gut (never in the rectal vesicle lumen). Mini-waves of vacuolar colour changes from the pink 'resting' state through olive, orange, yellow, red, and blue-in that order) occurred during the prey imbibition period (particularly in the later coxal droplet phase). However, essentially only two vacuolar staining properties are seen thereafter (i.e., pink and red). Their temporal relationship suggests that red vacuoles are the terminal 'sink stage' in a physiological cascade, pink being the penultimate stage (see chevrons). It would be tempting to conclude that: the early colour changes are either the secretion of enzymes/emulsifiers into the gut lumen or the facilitated uptake/breakdown of certain different prey components released in the lumen over time; and, the later colour transition are a shift from a focus of 'back-end' intracellular digestive activity (pink) to a focus of mainly egestive activity (red) in the gut epithelial cells. Immunocytochemistry confirmation is needed in follow-up TEM work.

The presence of some vacuoles in cells at $t=0$ confirms the mild inadequacy of the starvation period of 2 weeks used in this study already reported based upon gut lumenal changes by Bowman (2017b). Three weeks would have been better-however, this bias is 


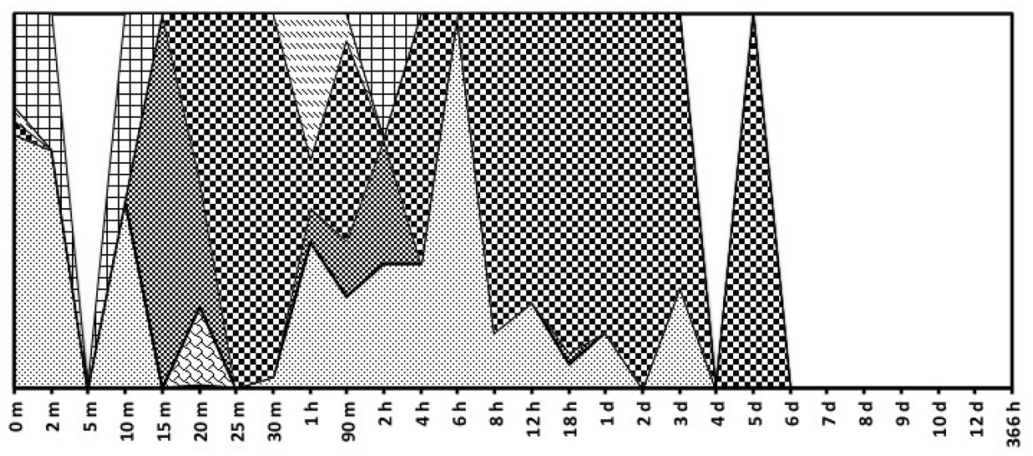

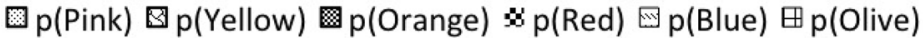
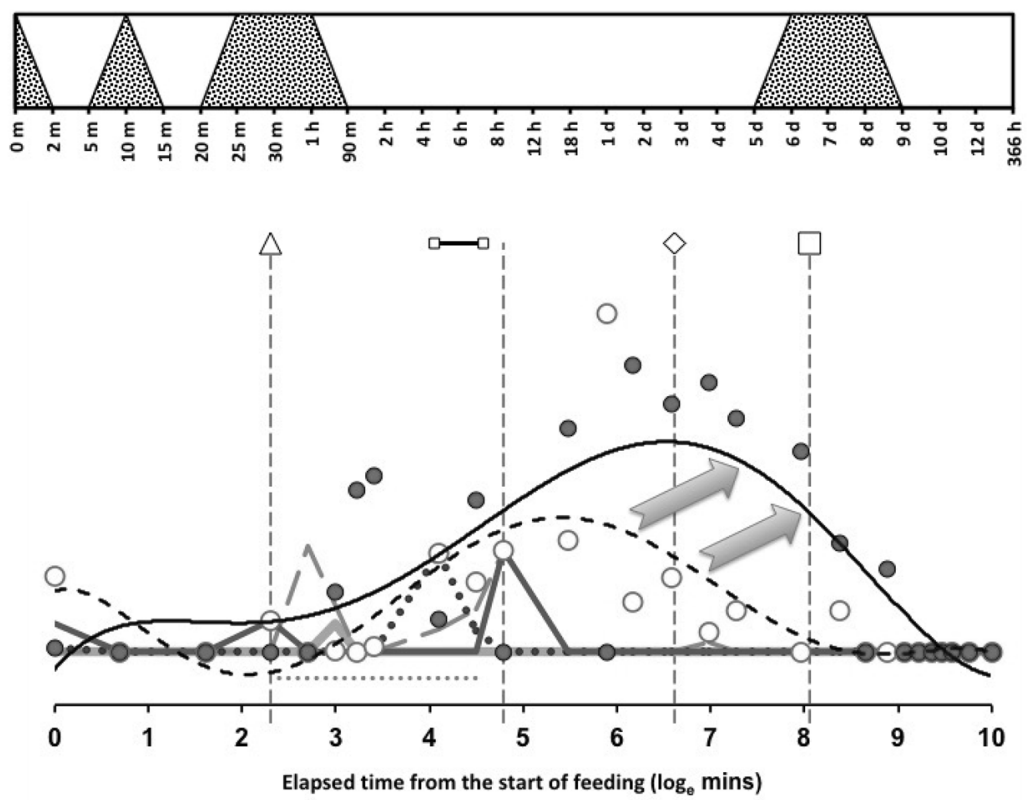

Fig. 16 Prey material staining in gut epithelial cell vacuoles-Pergamasus longicornis from feeding to fasting. Symbols and extra lines as in Fig. 7. Upper: stacked conditional probability of Mallory's Triple Stain stained vacuoles' colour $(=p($ colour $\mid$ vacuole $))$ in transverse sections estimated over all mid- and hind-gut regions (not including the rectal vesicle, and where there were vacuoles scored). White/transparent $=$ probability (no stained vacuoles present). Middle panel: rectal vesicle Mallory Triple Stain stained lumenal globular material (pink at $10 \mathrm{~min}, 25 \mathrm{~min}, 7$ days; olive at $0 \mathrm{~min}, 10 \mathrm{~min}, 25 \mathrm{~min}, 30 \mathrm{~min}, 8$ days; orange at $1 \mathrm{~h}, 6$ days; red at 7 days; blue at $1 \mathrm{~h}, 6$ days). Note none by end of prey imbibition, and distinct pulse around time of peak defecation (Bowman 2017b) after maximum guanine excretion (Bowman 2017a). Lower: joint probability of vacuole and colour staining $(=p$ (colour \& vacuole $)$ ) versus elapsed time from start of feeding on $\log _{e}$ min scale. Open circles and dashed black sixth order polynomial trend line $\rightarrow$ pink. Heavy grey solid line basally $\rightarrow$ yellow. Heavy dark grey solid line basally $\rightarrow$ olive. Twin peaked long dashed grey line $\rightarrow$ orange; solid grey circles plus solid black fifth order polynomial trend line $\rightarrow$ red. Dotted line basally $\rightarrow$ blue. Large grey chevrons indicate putative transfer as physiological focus switches from digestion to egestion 
low since: on most measures the gut in $P$. longicornis had reached a resting state (Bowman 2014, 2017a, b); vacuolation is rare and mild at $t=0$ (Figs. 7, 8, 9); and, enzyme histochemistry confirms a quiescent state (Tables 2, 3, 4 and 5) at this time. The gut epithelium in wild specimens may be acting like a 'hepatopancreas' storing previous or larger meals over a very long period, or alternatively gut processing in the wild is slower than in the laboratory. Blood digestion rate is affected by temperature in ticks (Teravsky 1951) and indoor temperatures in the $\mathrm{UK}$ at about $20^{\circ} \mathrm{C}$ are warmer than the net cooler temperatures found in forest soil litter (Paul et al. 2004) where pergamasids live. Absorbed but not completely catabolised vacuolar food stores would then act like a between-feedings reserve or buffer in P. longicornis much as vitellin does in ticks (Rosell-Davis and Coons 1989) and the blood meal itself in argasids (rather than fat or glycogen-Tatchell 1962).

The fact that vacuoles appear by $20 \mathrm{~min}$ (see Fig. 11)-whether they are secreted products for extracellular (intraluminar) digestion or indications of intracellular take-up and processing - refutes hypothesis (iii) in Bowman (2017b) for the control of digestion ('Check (b)'). Unless there is a big lag between digestion being triggered and intracellular changes being evident, then hypothesis (i) is also disfavoured. Occam's razor applies! Rather the close contemporaneity of micro-histological tissue changes to the $10 \mathrm{~min}$ point of maximum gut expansion supports hypothesis (ii) to be the most likely. Controlling signals thus would appear to emanate from stretch proprio-receptors in the mite gut as in insects (Langley 1976 and references within) rather than on the arthropods mouthparts (Wales 1976; Waladde et al. 1979). The burst of gut cell esterase activity changes (Table 2) matching the initial rise of square cells (Fig. 8) points to an early switch in cell metabolic status supporting this conjecture.

Vacuolar dynamics are stiff (Table 1). Early vacuoles themselves are not strongly positive for acidic phosphatase (Table 3) - a marker of lysosomes, so they must represent early prey fluid uptake but not its catabolism (at least not until up to $2 \mathrm{~h}$ after feeding). Whether later vacuolation i.e., after the 'digestive switch', are secondary lysosomes needs confirming in further enzyme histochemical work at later time slices. As cell sizes were not estimated it is impossible to directly test the claim of Reichenow (1918) that large cells are more active than small cells. Unlike the lumenal evidence in Bowman (2017b), vacuole changes do not clearly support food processing being slower in the posterior gut compared to that anteriorly. Furthermore, the 'hot spot' pattern of esterase activity early on in the midgut epidermis suggests cells are 'switched on' into high activity compared to the starved state during the rise of the appearance of square cells-this esterase activity increasing posteriorly within any one gut region. So the converse to Reichenow (1918) is supported. Any perceived slowness reported by researchers may simply be due to the large volume of potential food imbibed into the larger posterior parts of the mid-gut.

The proportion of cells showing large and small vacuoles is similar over most of the digestive period-metabolic activity per cell appears thus level as in Baker (1977). However, there is a noticeable lower proportion of cells showing small vacuoles around 4-8 $\mathrm{h}$ after the commencement of feeding (see central area of lower in Fig. 17). Thus this period may be the zenith of overall vacuole formation processes. If small vacuoles lead onto large vacuoles (as claimed in P. persimilis by Akimov and Starovir 1974; Starovir 1981a in A. casalis), and that large vacuoles lead onto egestive material as Tatchell (1964) describes in argasids (producing egestive alkaline haematin terminally in that case, producing the unseen refractive grains in the case of P. longicornis), then given the slow exhaustion of prey material for absorption in the gut lumen over time-see lower in Fig. 17this is exactly what one would see under a light microscope. That is: first small vacuoles; then small and large vacuoles; then mainly large vacuoles. It is not thought that the small 


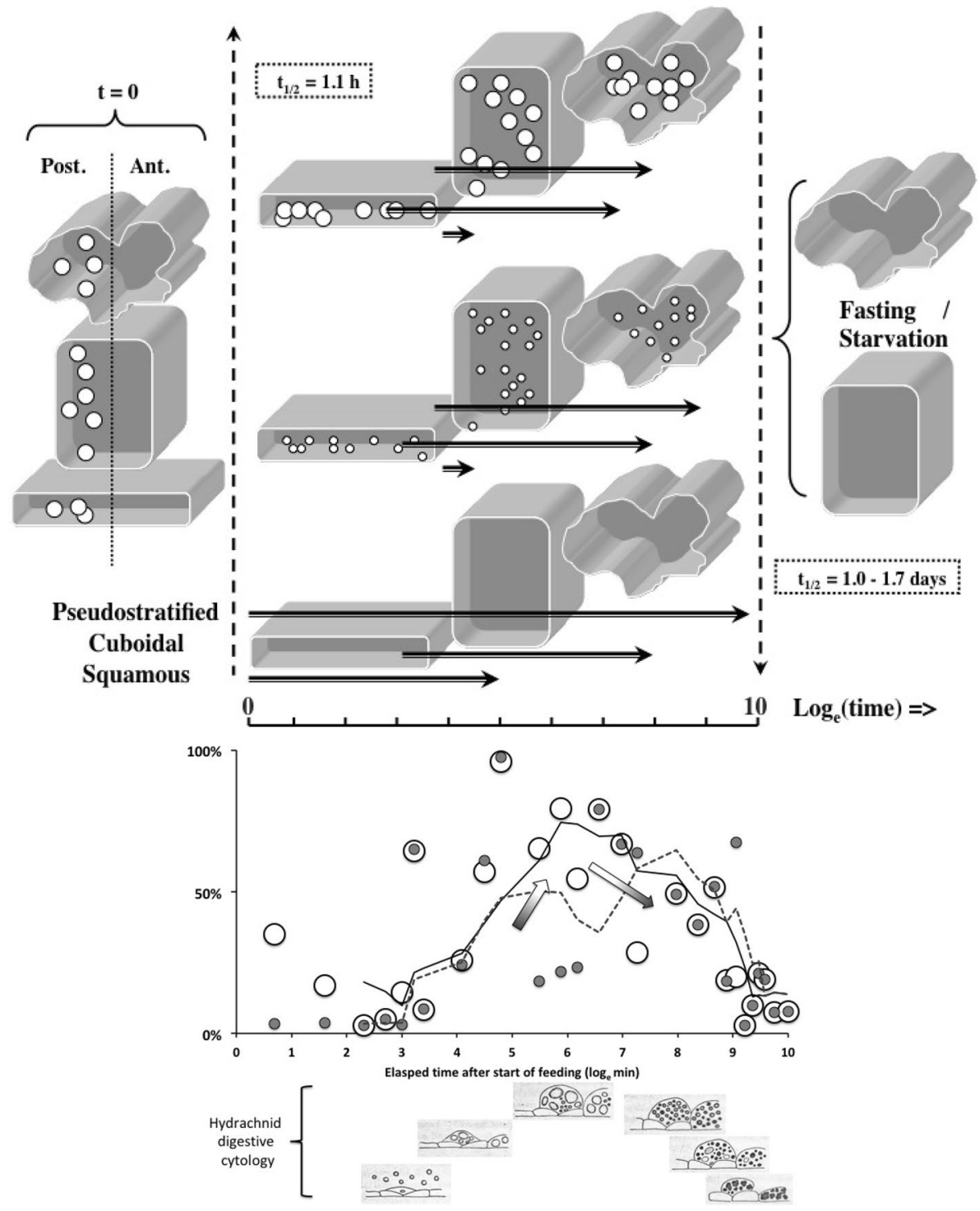

Fig. 17 Gut cells enlarge and vacuolate over time. Time course summary of epithelial tissue types (thin cells indicate squamous epithelium; square cells indicate columnar/cuboidal epithelium; jigsaw-piece cells indicate transitional pseudo-stratified epithelium) and vacuolation of cells on $\log _{e}$ scale after the start of feeding on larval dipteran prey in Pergamasus longicornis. Upper: overall summary of relative cells sizes, shapes and vacuolation. Dashed vertical arrows and half-lives are of vacuolation (on left) and devacuolation (on right) as the cells agefrom Table 1. Bold arrows represent time period of which that cell type is present. Size of vacuoles (none, small, large) up the page, indicating progressively more digestive activity. Shape of cells match labelling (thin, square, jigsaw). Lower: time course graph of proportion of cells with vacuoles over time annotated by cytology results from Bader (1938) as displayed by Mitchell (1970) in temporal order (@Taylor \& Francis Ltd, http://www.tandf online.com, with permission). Fourth order moving average dotted line and small grey circles $\rightarrow$ 'small vacuoles'; Fourth order moving average solid line and large open circles $\rightarrow$ 'large vacuoles'. Note general lack of small vacuoles per cell around 4-18 h after the start of feeding (i.e., after the egestive switch) and the late predominance of small vacuoles to large ones as food absorption occurs up to 'switch' (and peak large vacuole predominance), followed by conversion of large vacuoles into small vacuoles (of a different type?) and tail-out phase during egestion. Note consilience with hydrachnid digestion results leading to egestive excretory waste 
vacuoles reflect the absorption of different prey material than that into large vacuoles (as with albumin and haemoglobin, respectively in the tick B. microplus Lara et al. 2005). Of course, this might in fact be the case in P. longicornis - further immunohistochemical work is needed to see if a specific clathrin 'coated' pit mechanism like that in ticks (Raikhel 1974b; Coons et al. 1986) exists which transports material into vacuoles. Notwithstanding such follow-up, vacuolar specialisation may be a moot point, as Sojka et al. (2016) show in ticks that despite differential transport during heterophagy to deal with heme toxicity, host proteins are digested by a common multi-enzyme proteolytic network to yield suitable amino acids for metabolism and anabolism. To paraphrase Alain de Lille in 1175: it is not that "All roads lead to Rome" - it is that everything leads to the haemocoel (Hefnawy 1972a).

After this 4-8 h 'switch' point there is observed a surfeit of small vacuoles. What could these be? Firstly they could be small vacuoles (never destined to grow larger because lumenal food is being exhausted - and therefore large and small vacuoles remain in synchrony as they both decline in prevalence). Or secondly, they could be small vacuoles associated with egestive material production (from the preceding pulse of large vacuoles). That is, that the late small vacuoles are qualitatively different physiologically to the early small vacuoles-i.e., they have a different metabolic focus (see Fig. 18, middle row and also Balashov and Raikhel 1976a). This needs confirming in follow-up histochemical and TEM work. Certainly the results of Bader (1938) redrawn by Mitchell (1970) show the same 'tailing out' phenomenon of small vacuoles as large vacuoles disappear over time and egestive material appears and accumulates. Mapping the order and form of these hydrachnid cytological results into lower in Fig. 17 matches the vacuolar results of P. longicornis well (albeit given a slightly different overall time window for the different mite species). Further consilience is seen, in a linear system sense, of the 'echo' of the dynamics of the small vacuoles being turned into large vacuoles, by the secondary rise of the (different?) small vacuoles as the large vacuoles decline (shaded arrows), which in turn matches the temporal location and pattern of cellular disintegration (gut 'lumenation') and lumenal grain appearance. The conjecture is consistent. Could alternatively this be the first evidence of a 'slow digestion' intracellular phase versus a 'fast digestion' intracellular phase in mesostigmatids? If so it is particularly posteriorly-Fig. 13, lower.

The half-life of vacuoles appearing is slower than the half-life of granular material being imbibed. In fact the half-life of vacuoles appearing is of the same order as that of globular material disappearing from the lumen (Bowman 2017b—suggesting that perhaps extracellular (intraluminar) digestion of the granular-ingested liquified-prey tissues may be happening first in the lumen-early-on in the digestive process- to generate more 'assimilable' material for the cells to take up (see below discussion section on 'Free' cells also). This is feasible as Hughes (1954b) notes in Ixodes ricinus that erythrocytes are digested as soon as they enter the gut, and according to Tatchell (1964) blood corpuscles only disappear by the sixth day after feeding in argasids. However, Reichenow (1918) suggests such phenomena are an obscuring bacterial infection in the gut of Liponyssus, and Baker (1971) ascribes salivary activities as the origin of erythrocyte breakdown in Xenopacarus africanus. A variety of enzymatic activities are known in the extruded digestive juices of other arachnids (Mansour-Bek 1954; Yonge 1954a, b) facilitating the resorption of food (Krijgsman 1954), so biochemical assays over time of prey degradation in situ could verify this conjecture in P. longicornis. Similarly, a check could also be made whether coxal fluid has any extra-corporeal lytic/coagulative abilities as in argasids (Balashov 1964). As feeding stops between 56 and 96 min after it starts (Bowman 1987a) and peak vacuolation is 

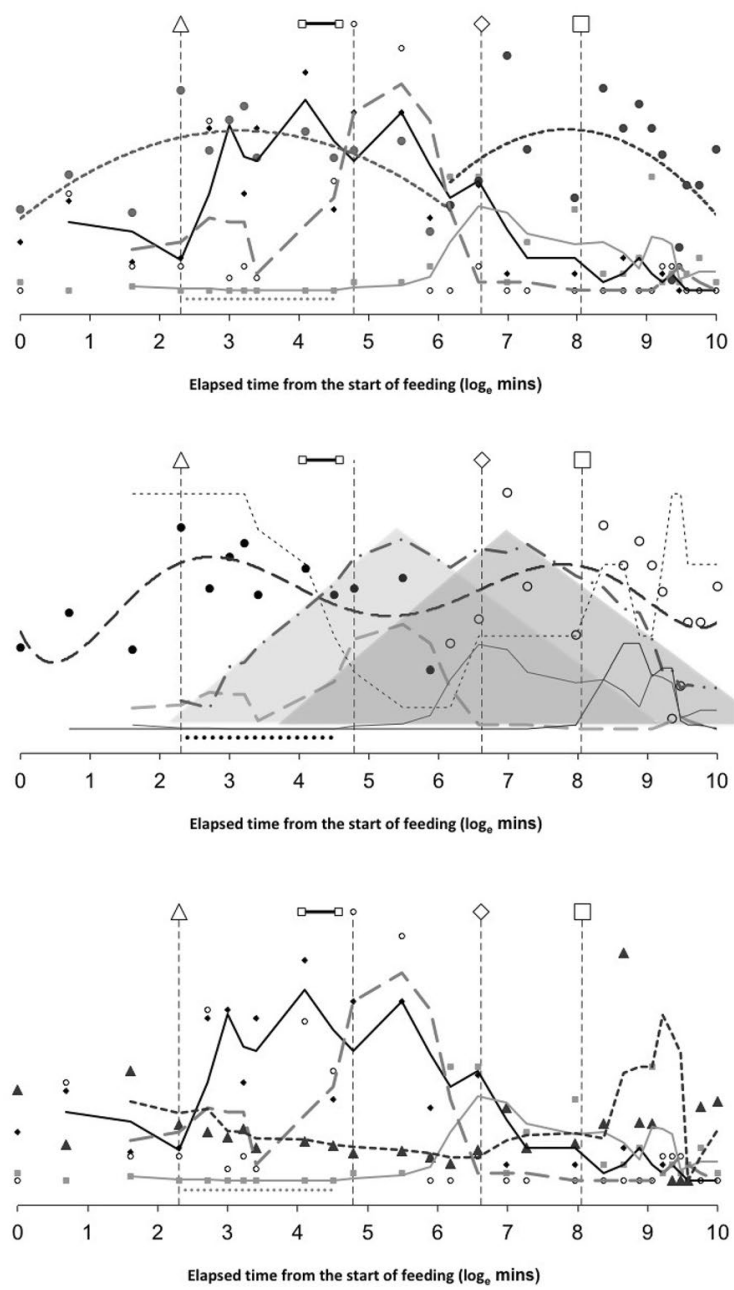

Fig. 18 'Vacant lumen' score (estimated as lumen score - visible food score - waste score). Upper row: 'Vacant lumen' score-solid circles superimposed upon lumenal granular, globular and refractive grains score traces from Bowman (2017b). Up to and including $8 \mathrm{~h}$-first dashed parabola. $8 \mathrm{~h}$ and more-second dashed parabola. Note decline (around central knot point of $8 \mathrm{~h}$ ) indicating a drop in spare lumen space as mid-gut active cellular fraction predominates. Peaks of two parabolas generally match the two pulses of clavate cells observed (see Fig. 12) either side of it. Middle row: 'Vacant lumen' score-dashed sixth order polynomial trend confirming dip centrally (around $4 \mathrm{~h}$ ), superimposed upon lumenal globular, lumenal refractive grains, Malpighian tubule guanine and rectal vesicle expansion/contraction score traces from Bowman (2017a) and Bowman (2017b). Solid circles-up to and including $8 \mathrm{~h}$. Open circles- $8 \mathrm{~h}$ and more. Dashed and dotted line is quartic trend line for average of (small vacuoles per cell and large vacuoles per cell) treating each cell type equally. Note dip in relative cellular vacuolation around 8-12 h confirming middle panel of Fig. 17 as shift from digestion to egestion progresses (and small vacuole physiological focus changes?). The lack of space for any 'vacant' lumen is driven by cellular growth as prey food is absorbed and processed intracellularly vacuoles. Shaded areas indicate the two possible physiological foci for vacuoles overlapping in time (left 'fast' absorption/digestion, right 'slow' intracellular digestion/egestion). Lower row: 'Vacant lumen' score scaled by expansion/contraction score from Bowman (2014)—solid triangles plus dashed three period moving average trend line. Note how the relative opportunity for vacant lumen space (sharp peak to right) occurs around the time of lumenal refractive grains moving into the rectal vesicle and the expansion of Malpighian tubule guanine consuming idiosomal volume 
at $90 \mathrm{~min}$, there were insufficient data points to check if digestive activity reduces on 'prey detachment' as in ticks (Tarnowski and Coons 1989).

Table 1 shows that vacuoles disappear faster $\left(\hat{t}_{1 / 2}=1-2.4\right.$ days $)$ than the disappearance of square cells $\left(\hat{t}_{1 / 2}=4.9\right.$ days $)$ and faster than that of gut overall contraction $\left(\hat{t}_{1 / 2}=4.4\right.$ days). All of these span the half-lives of comparable processes in the early phases of food elimination in argasid ticks (Table 7 herein and Table 2 in Bowman 2017b). They are also consilient with the modest half-life of 2.3-2.7 days for overall food elimination and the elimination of imbibed granular material in P. longicornis, and the half life of 2.6 days for the relative amount of food remaining over time in the gut of wolf spiders (i.e., $\frac{\ln (2)}{0.2667}$ from Fig. 4 in Nakamura 1972). Square and jigsaw-piece cell vacuolation is thus interpreted as evidence for intracellular digestion in $P$. longicornis - perhaps via proteolysis as in I. ricinus (Sojka et al. 2008; Franta et al. 2010).

Complete cell vacuolation would be expected by $5.5 \mathrm{~h}$ after the start of feeding (from the results in Table 1). However, initial gut emptying does not in fact start until $12.5 \mathrm{~h}$ post start of feeding (Bowman 2014) when granular lumenal contents begin to disappear (Bowman 2017b), so an extended period occurs when the gut epithelium is maximally vacuolate (and dominated by predominantly large vacuole carrying cells of both tissue types). Refractive grains start to appear in the gut lumen by $4 \mathrm{~h}$ post feeding (Bowman 2017b) with a half-life of appearance of $6.3 \mathrm{~h}$ (Bowman 2017b), suggesting that during this extended period of vacuolation, egestion gets under way. That is, prey food is being converted to waste. It is tempting to conclude that vacuoles are being facultatively converted (by an unseen body process) into the presence of lumenal refractive grains. Perhaps solubilised material in these vacuoles is catabolised and stored intracellularly as glycogen granules (known in pycnogonid gut cells-Schlottke 1933, and in tick intestinal cells-Raikhel 1974a) whilst the residues are shed as residual bodies (or 'pigment' granules)? Such waste material is stored as inert repositories in Demodex - Stromberg and Nutting (1979). Further enzyme histochemical localisation would be useful such as to histochemically localise aminopeptidase - known at the border of vacuoles in tick gut cells (Tatchell 1964), say by using the $\beta$-naphthylamide method of Burstone and Folk 1956 on the gut epithelium sections. Endopeptidases are a part of the acidic peptidase network (to break up proteins into peptide chunks) which are induced upon feeding in ticks (Horn et al. 2009; Franta et al. 2010) and are important in parasitic mites (Nisbet and Billingsley 2000)_Psoroptes ovis which rely upon acid peptidases intracellularly for digestion (Hamilton et al. 2003). Electron microscopic histology could confirm such conversions and where they might be happening in P. longicornis. In fact it may not be a simple catabolic process-as the half-life of vacuole disappearance is much slower than the appearance half-life of lumenal refractive grains (Bowman 2017b). Rather it may be a cascade matching the Malpighian tubule guanine generative process detected in Bowman (2017a). The kinetics of TEM changes in mid-gut cells would help here.

Given the estimated half-lives, the complete disappearance of cell vacuolation would be expected by $65.3-114.9 \mathrm{~h}$ after the start of feeding. On this basis, thus P. longicornis histologically effectively engenders $0.21-0.36$ gut emptyings per day. These figures are longer than the worst case total feeding cycle time of $52.5 \mathrm{~h}$ quoted by Bowman (2014) modelling gut expansion/contraction - thereafter being egestion-again confirming that gut gross size on its own is a poor overall predictor of exact feeding status. However, gut histology on its own is a better measure than even Malpighian guanine excretion. Producing the most extreme estimate of decline $(* *$ in Table 1$)$ based upon the results of this study would indicate no gut cell vacuoles by about 9 days from the start of feeding ( $=$ the putative complete cycle of feeding, digestion, egestion and excretion in Bowman 2017a). Consilience 


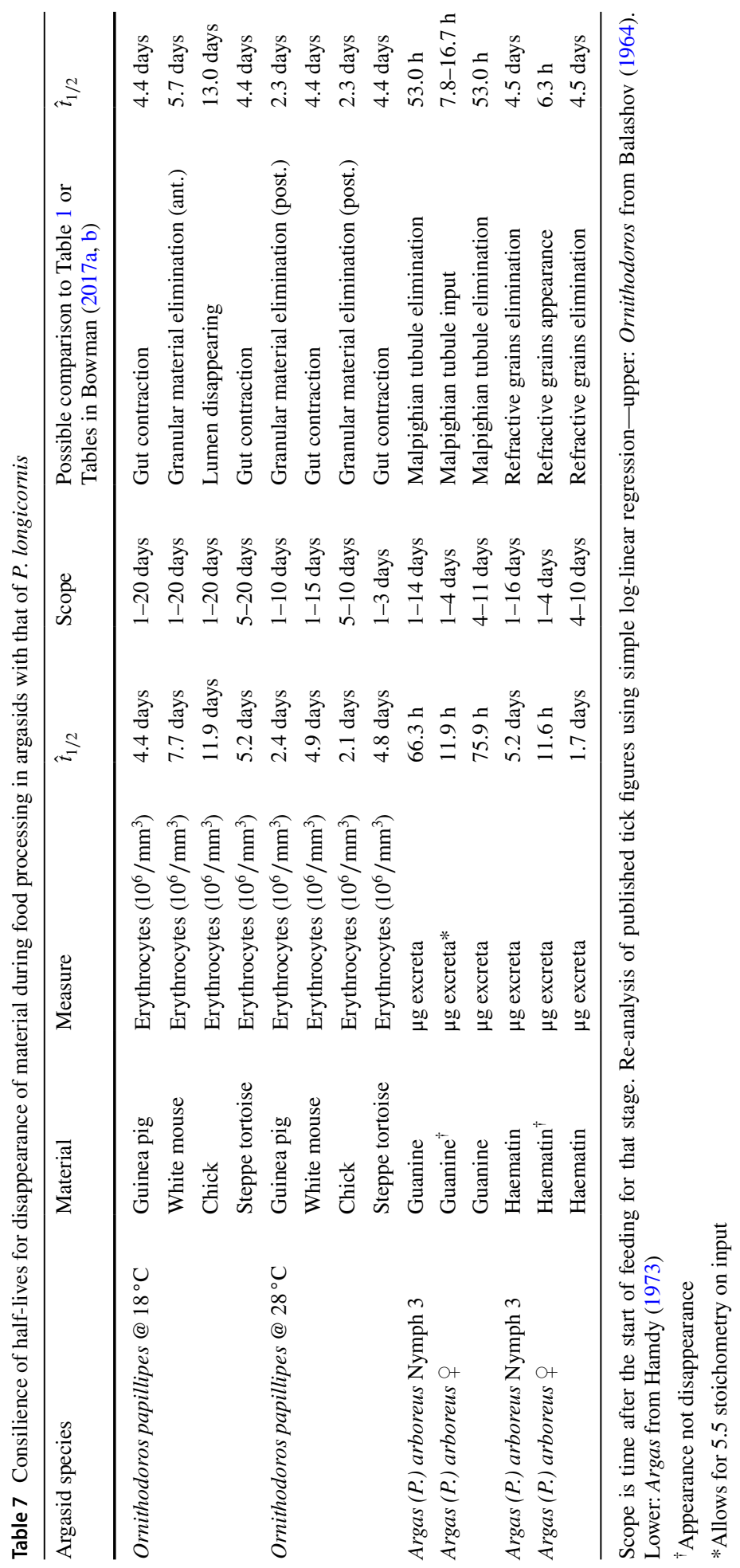


occurs ! This is equivalent to 0.11 total gut emptyings per day-and is an order of magnitude less clearing than in phytoseiids (Akimov and Starovir 1974 and references in Bowman 2014). This elapsed time is well before hunger/starvation likely commences at 10 days (after the start of feeding-Bowman 2017a) or the up to 15 days which may be needed to completely clear the idiosoma of excretory material (Bowman 2017a). The gut epithelium in wild specimens must therefore be handling or storing much larger amounts of prey foodstuff than a second instar D. melanogaster larva, or gut processing is slower in the cool temperatures of temperate forest litter. Whilst 2 weeks fasting in laboratory conditions might be just about sufficient, given the five half-life values for square cell disappearance and gut contraction, around 3 weeks may be needed in the field for full prey clearance.

Oboussier (1939) independently describes the vigorous secretory activity of epithelial cells ("die lebhafte sekretorische Tätigkeit ihrer Epithelzellen") in the wide cul-de-sac pouches of the acarid stomach without describing two distinct cell types. These secretory cells appear to match the 'square cells with vacuoles' found in P. longicornis. Oboussier (1939) states that secretions accumulate within such cells in T. dimidiatus, they then swell club-like ("kolbenformig") outwards into the lumen-like the 'clavate' cells of P. longicornis - until the upper part of each cell finally dissolves together with the secreted substances and merges into the lumen. However, in P. longicornis there is no clear evidence of two types (cf. digestive vs. secretory) of 'square' cells nor of two types of 'jigsaw-piece' cells whether in size or vacuolar characteristics. On the contrary, the two types of main cell found appear to be just two interchangeable variants of the same digestive cell (post the epithelial cell being 'thin' as the gut epithelial tissue changes its overall form). The popular state for most of the gut epithelium is the 'square' cell columnar tissue, the 'jigsaw-piece' like irregularly shaped and tessellated cell is simply the base state for the highly contracted alumenate anterior caeca before digestion and is the frequent state during periods of high digestive activity and very active cell growth occluding the lumen (e.g. in the hind-gut practically throughout the whole period of this study).

No evidence for discrete cells such as the digestive, excretory and basal cells of the actinotrichid Dactylochelifer (Gilbert 1952) was seen. Rather, a single fundamental type of gut epithelial cell was seen as in M. murismusculi (Filimonova 2001 and Syringophilopsis frigilla (Filimonova 2009). On starvation no secretory cells (as described by Roesler 1934; Neumann 1941; or later Soviet-at-the-time researchers) were observed either. Furthermore, no specialised 'egestive' cell was found to match the rough digestive cells described by Akimov and Starovir that degenerate and release crystals (= refractive grains herein?). Indeed, no cell type or tissue form was observed uniquely associated with the period when refractive grains were predominant in the gut lumen in $P$. longicornis. Nor could these refractive grains be observed inside any gut cells. On the face of it, these egestive grains appear to formed in situ in the gut lumen or if secreted from gut cells (like the birefringent pigment spheres of $L$. saurarum Reichenow 1918) they are much smaller intracellularly (and impossible to easily discern with light microscopy) than say the large excretory guanine crystals found in the Malpighian tubules. Perhaps egestive wastes, pigments, spherocrystals and iron granules are ejected into the lumen of the diverticula as in scorpions (Goyffon and Martoja 1983)—where they disintegrate into a dust of micro-crystals? The lack of clear blackish excreta bodies inside the cells as found in blood-feeding ticks (Till 1961) makes common sense as a pergamasid does not have to deal with large amounts of insoluble heamatin. Transmission electron micrography of gut cells would be useful in determining the origin of the refractive grains and determining if they are residual bodies (Raikhel 1978) or metal containing spherites (as in isopods Prosi et al. 1983) or even micro-inclusions of guanine- $\breve{S}$ ustr and Hubert (1999), originally formed in the gut cells. 
Both nitrogen and iron are excreted during the blood sucking process in ticks-Kitaoka (1961b). After the switch from net gut-filling to net gut-emptying (i.e., after ingestion and most absorption has occurred) in P. longicornis, given that both the levels and the changes in prevalence of vacuolated cells fall in line with the levels and changes in the occurrence of refractive grains in the lumen, it is tempting to conclude that both vacuolation and the appearance of the refractive grains represent the same bursts of late digestive activity in the epithelium.

In conclusion, no evidence has been found of fundamentally different types of gut epithelial cells in P. longicornis.

\section{‘Clavate' and 'Free' cells}

Clavate and free-floating (Fig. 12) cells occurred periodically but rarely during feeding and digestion, and it is hard to tell the difference between them definitively in histological sectional views. Neither were scored as carrying vacuoles nor any deposits of faecal material as reported by Bader (1938) in hydrachnid mites. However, free cells appear earlier on in time than clavate cells.

The general occurrence of clavate cells appears to temporally match the presence of 'jigsaw-piece' cells and so one conjecture is that they could simply be a lumenally protruding variant of such. That is, just extensions into the lumen as in Psoroptes ovis (Mathieson and Lehane 2002) and the swollen taller cells in L. saurarum after blood imbibition (Reichenow 1918). In particular, clavate cells are strongly correlated with the appearance of large vacuolated 'square' cells during the central digestive period (see Fig. 12) and so could simply be a lumenally protruding (early) variant of these (like in argasids (Tatchell 1964). There is also a notable peak in them around the best estimate of the time of initial gut emptying later at $12.5 \mathrm{~h}$ (from Bowman 2014) when refractive grains start to appear in the lumen (Bowman 2017b). These later appearing clavate cells may represent those described by other authors as 'secretory' cells at the beginning and end of digestion (Akimov and Starovir 1974). However, the timing of their occurrence does not suggest that they are digestive enzyme secretory. Given their relative predominance (see upper panel in Fig. 19) over the 90 min-3 days post-imbibition period, perhaps their degradation (apically?) releases such refractive grains as a micro-crystalline 'dust' of residual bodies from what were originally square cells? Further detailed TEM work is needed to test this conjecture. Clavate cells would of course increase the absolute surface area of the gut epithelium in contact with the lumen and so synergise any uptake (or any release) of material at these two critical time periods. This is consilient as they appear at exactly the times of maximum 'vacant lumen' space (Fig. 18, upper row) when deliberate adaptive cellular extension (for either uptake or release) would be best!

In a serial sectional study it is not possible to be absolutely sure that the 'free' cells in $P$. longicornis are actually free of the rest of the epithelium. Even in well studied ticks claims of the budding-off of mid-gut epithelial cells are contradictory (Chinery 1964). However, the descriptions of cells being cast off leaving a thin epithelium of cells (Hughes 1954b) or cells being released from the midgut epidermis in various ticks (Section V. in Arthur 1965 ) does match the temporality in P. longicornis. Free cells could represent the cut off apices of other cells as in Mitopus morio (Phillipson 1961 or may be true cast-offs from constricted smooth digestive cells reported by: Akimov and Starovir (1977) in A. andersoni; by Starovir (1981a) in A. casalis; and, by Starovir (1981b) in A. umbraticus. The fact that the occurrence of free cells does not match the occurrence of clavate cells over time 

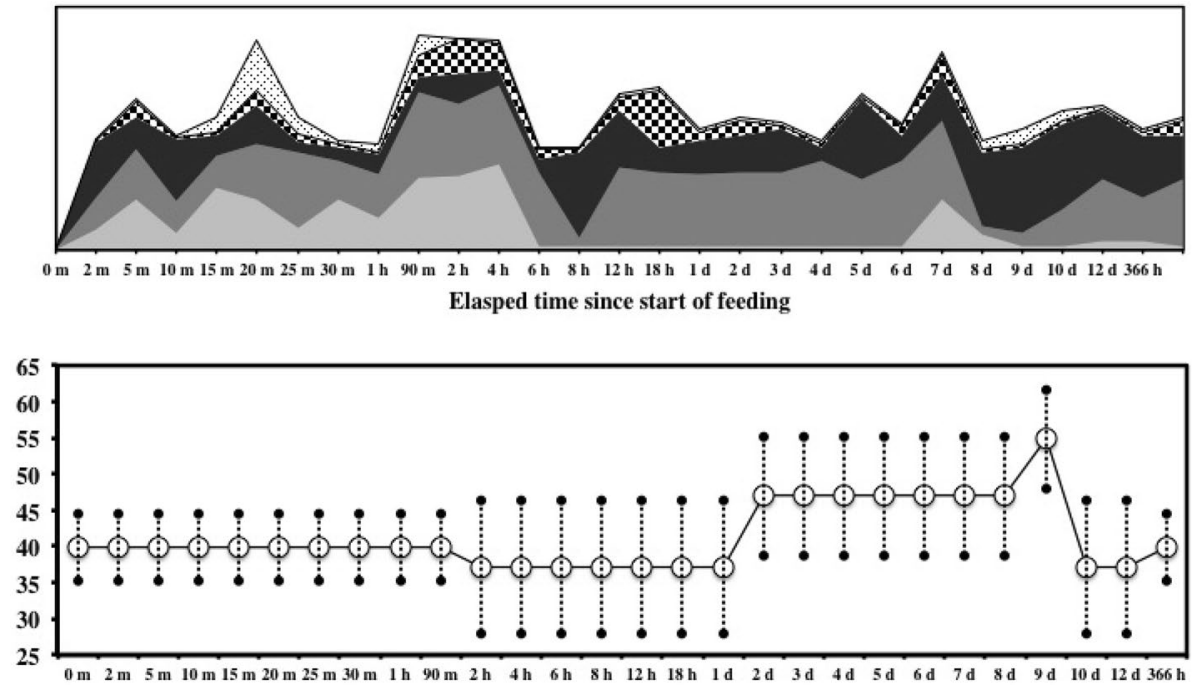

Fig. 19 Cell types and protein content are not related in Pergamasus longicornis. Upper: stacked timecourse summary of cell types against time point of collection after the start of feeding on larval dipteran prey in Pergamasus longicornis. Pale grey $=$ Thin cells (simple squamous epithelium). Medium grey $=$ square cells (cuboidal or columnar epithelium). Solid black = jigsaw-piece like irregularly tessellated cells (stratified or transitional pseudo-stratified epithelium). Black and white small square hatching = clavate cells. Pale stippling $=$ free cells. Note simple squamous epithelium $2 \mathrm{~min}-4 \mathrm{~h}$, and predominance of pseudo-stratified/transitional epithelium in fasted/starved state $>8$ days. Free cells only seen during act of feeding (56-96 min Bowman 1987a). Clavate cells occurrence matches digestive and egestive phases. Lower: protein content ( $\mu \mathrm{g}$ BSA equivalents) from Bowman (1985). Large open circles plus bars with solid ends $=$ mean $\pm 95 \% \mathrm{CI}$ over both genders categorised for each observed nutritive state ([-:-], [dark:-], [-:white ], [dark:white ]) of the 101 mites at collection. Protein content values displayed over feeding to fasting for period of maximum posterior probability of that state (from Bowman 2017a Fig. 21). Note overall body protein content rises over $1-10$ days $(\ln (\mathrm{min})=7.27-9.57)$ with a distinct peak around 9 days $(\ln (\min )=9.47)$ from the start of feeding before a dramatic fall

(compare Fig. 12, upper and lower) suggests that free cells are probably not the sectioned cut-off tops of clavate cells, but represent truly dehisced cells. Further, since the free floating cells in $P$. longicornis only appear during the coxal droplet phase of great gut expansion with watery prey material-it is tempting to believe that they are in fact dehiscent cells as in the gut of Psorpotes ovis (Mathieson and Lehane 2002) and the gut of B. bacoti (Hughes 1952). They cannot be osmoregulatory cells per se if they are disconnected from the gut, however, they might be cells indicative of a burst of ammonia waste production if pergamasids use the same ammonia vaporising water excretion/recycling system as in isopods (Hoese 1981). The 'free cells' disappear after feeding stops just at the rise of globular material in the lumen. Their degeneration might be releasing emulsifiers into the lumeninvertebrates need such (Vonk 1962). However, their lack of overlap with globular lumenal contents decries this idea. If they were direct emulsifying agents as in arachnids (Collatz and Mommsen 1974), one would have to pose a delay of around $1 \mathrm{~h}$ in their action.

Oboussier (1939)'s postulated secretory function (see above) better matches the time course of 'free cells' in P. longicornis and their internal 'bead-let' structure. Could they be analogous to the basophilic cells that produce exoenzymes in scorpions (Goyffon and Martoja 1983)? Are these pergamasid 'beads' zymogens ? Are these cells like the F-cells 
of crustacea (Gibson and Barker 1979)? Is the first phase after the 'digestive switch' in $P$. longicornis an alkaline lumenal process (argasid guts are slightly alkaline-Popow and Golzowa 1933)? For sure in any animal that can can fast for a long period of time, it requires the active proteins/enzymes in digestive fluids to either be ready as a gut lumen colloid in the unfed state (Hughes 1954b) or be synthesised and deployed rather quickly and be reasonably stable over longer times-Walter et al. (2017). Free cells only appear for a brief time and may release enzymes for extracellular (intraluminal) digestion (from their internal bead-lets?). In that way they may match the vesicle-discharging secretory cells of spiders (Fuzita et al. 2016b) implicated in prey liquefying. Such a 'one-off' release into the digestive fluid of spiders contains all the enzymes needed for extra-oral digestion during the entire (single) prey-feeding cycle. The strong alkaline phosphatase in the lumen at 2 $\mathrm{h}$ post feeding (Table 4, lower) matches the appearance of globular material in the lumen Bowman 2017b) suggesting that the two may be linked. Is the globular material in fact evidence of such a produced (alkaline) colloid? As the lumenal activity in P. longicornis is well after prey imbibition has stopped and was not present in the lumen earlier, it cannot arise from imbibed prey tissues (nor a salivary gland source!). Alkaline phosphatase (along with esterase and acid phosphatase) is known in the gut fluid of spiders where it is thought to play a role in intermediary carbohydrate metabolism (Couch and Benton 1971). This alkali phosphatase might be involved in calcium processing and spherite production as in other arachnids (Ludwig and Alberti 1988a). Fuzita et al. (2016b) poses an alkaline extraoral digestive phase for prey tissues in the digestive fluid of the midgut diverticular lumen in spiders. It would be tempting to conclude that alkaline phosphatase is being dumped into the gut lumen in the early digestion phase after feeding has stopped in P. longicornis in the same manner. This could be by the consequence of the lysis of the 'bead-let carrying' free cells in P. longicornis. Careful enzyme histochemical and TEM intensive tracking of these free cells and gut lumen chemistry over this critical period between 30 min after feeding up to the time of the 'digestive switch' at $2 \mathrm{~h}$ is needed in detailed follow-up work to confirm or refute this.

Proteases are known in ticks (Reich and Zorzópulos 1978). Agyei et al. (1991) describes solitary B. microplus tick mid-gut epithelial cells which move into the lumen and disintegrate during the slow digestive phase. Not only was acid phosphatase and non-specific esterase activity present in them, but the pattern of appearance of these cells paralleled the reported protease activity in the mid-gut lumen. For sure, unfed ticks show negligible proteolytic activity in the unfed gut (Bogin and Hadani 1973; Akov et al. 1976). Cysteine cathepsins play an important role in protein digestion in scorpions-Fuzita et al. (2016a). Varroa jacobsoni (Mesostigmata) has a cathepsin-like protease localised in its mid-gut (Barabanova 1984a). Protease complements are known to indicate environmental adaptations in mites (Barabanova 1973; Bowman 1981, 1984b; Erban and Hubert 2010a; Holt et al. 2013), so are a good indicator of physiology. Intriguingly, whole gut epithelial cells only slough off into the lumen like this in the first continuous phase of digestion during slow engorgement in attached Dermacentor variabilis ticks (Tarnowski and Coons 1989) too. Whole cells tear away from the epithelium to secrete digestive enzymes in a holocrine manner in Ixodes spp. (Grigor'eva 2003). So, given that free cells only appear during the concentrative phase of pergamasid feeding and are not strongly correlated with the presence of any other cell type (or any type of lumen contents), it is tempting to conclude that they may be relatively undifferentiated $P$. longicornis gut cells who break-up and release enzymes (perhaps including lipases, or proteases-Tatchell et al. 1972) into the lumen to begin the extracellular breakdown (or augment any extra-corporeal tissue breakdown) of the imbibed food during 'prey attachment' i.e., before discard of the empty prey cuticle 
(Bowman 1984a, 1987a). They would thus augment extra-corporeal proteases from saliva acting upon ingested food of a tougher nature. This could be looked for in follow-up work.

Is it that 'free cells' are spawned from the same type of cell that becomes a 'thin cell' by stretching? Akimov and Starovir (1977) state that immature gut cells might be cast off into the lumen in A. andersoni-which would fit this idea. Acid phosphatase is found in the tick B. microplus gut (Gough and Kemp 1995)—which would fit this idea. Such enzymes would then facilitate the continued consumption of fluids (Bowman 2017b) during pergamasid 'prey attachment'. Bowman (1987b) already detected most hydrolases (but not common endopeptidases) in P. longicornis extracts. Exopeptidases more systematically disassemble prey peptides by breaking the bonds of terminal amino acids step by step. Amino acids are found in all types of mites (Butler and Tonn 1963). Protein hydrolysis occurs in acarid and glycyphagid guts (Akimov 1980) with a variety of proteases having been demonstrated in situ in the gut of Lepidoglyphus destructor (Erban and Hubert 2011). The mesostigmatid A. casalis is also known to produce a variety of enzymes too (Barabanova 1980a). Proteases have been found in hungry phytoseiid predators like A. andersoni and Anthoseius malicolus (Barabanova 1980b). Chymosin, pepsin, trypsin, invertase, lipase, inulase, amylase and catalase are known from the digestive glands and intestinal diverticula of various scorpions (Serfaty 1941a, 1942a, b; Bardi and George 1943). Catalase activity changes during the course of digestion (Serfaty 1941b). Catalase degradation is probably related to a hepatic-like function of ferric compound sequestration (Goyffon and Martoja 1983). Amylase and triacylglycerol lipases are found in the secretory vesicles of midgut cells in spiders-Fuzita et al. (2016b). So, could these 'free cells' in P. longicornis be a similar holocrine secretory mechanism? The apparent delay in effective action would fit the idea of acting upon relatively tougher ingested material (with salivary enzymes dissolving the easily broken down material). Definitive micro-biochemical evidence of extracellular digestion in situ is needed (together with TEM examination if the free cells are the same cell type as thin and square cells or not).

Note that, for sure by virtue of their occurrence over time, the 'free' cells in P. longicornis cannot represent the egestive detached digestive cells of Agyei and Runham (1995). Thus the idea that extracellular digestion is the incidental consequence of the elimination of waste products from cells (primarily concerned with intracellular digestion)-Gordon (1972) and Morton (1979) — is not supported in pergamasids.

\section{Functional synthesis}

Considering all the above, a functional synthesis building in all previous work on $P$. longicornis is offered below.

\section{Gut overall expansion, contraction and 'lumenation'}

Overall, the gut and excretory system in P. longicornis is very stiff dynamically (Bowman 2014, 2017a). There are very slow processes e.g. Malpighian guanine production and excretion (Bowman 2017a).There are multiple high speed processes: start of coxal droplet production (Bowman 2014); initial gut lumen appearance and prey ingestion (Bowman 2017b); and, the appearance of a simple squamous epithelium of thin cells (Table 1). However, the bulk of the pergamasid gut system has the medium time-scale dynamics of a tightly coupled tissue. The mid- (and hind-) gut cell is in fact a multifunctional moiety capable of both secretory and digestive activities (like in ixodids-Agyei et al. 1992). 
There is no strong evidence-see upper panel in Fig. 19-that the gut epithelium clearly behaves rhythmically over time as reported in ixodid ticks (Grigor'eva 2003, 2005, 2006) or as reported in phytoseiids like $P$. persimilis (Akimov and Starovir 1974) or A. andersoni (Akimov and Starovir 1977). This is not precluded but rather, the pattern looks much more like an artefact resulting from the between mite destructive sampling method used in this study.

There is clear strong cellular evidence (e.g. the zenith of 'thin' cells-Fig. 14) for a 'digestive switch' at $2 \mathrm{~h}$ from gut filling predominating to gut emptying predominating ['Check (c)']. The estimated half-life of gut expansion broadly matches that for square cell appearance and that for vacuolation (together with that for thin cell disappearance) much better than the half-lives for thin cell or lumenal appearance or that for prey food input (Bowman 2017b). This suggest that the major determinant of overall gut size increase is epithelial cellular expansion/growth as a coherent organ. Such expansion/growth can only be through the uptake of ingested fluidised prey material and its assimilative conversion into body tissues. How to test this conjecture?

Cellular predominance (or relative cellularity $\equiv \frac{1}{\text { lumenation }}$ ) was estimated at each time point $t$ as:

$$
\frac{\sum[\text { expcontract }]}{\sum[\text { lumen }]},
$$

(both measures pre-standardized so this is a $z$-score ratio in effect) using the gut expansioncontraction score from Bowman (2014). Simple algebraic manipulation of area $\left(=\pi \cdot r^{2}\right)$ and perimeter or surface area $(=2 . \pi . r)$ of an ideal circular gut part transverse cross-section shows that lumenation $\equiv$ (relative surface area $)^{2}$ between the total gut and the cellular portion of the gut (this result is equivalently lumenation $\equiv$ relative radii ${ }^{2}$ ). So if cells differentially degenerate for that total size of gut, a big increase in relative surface area should be seen. The fact that Fig. 20 lower shows a differential increase in lumen after the zenith of cellular digestion and that the half-life of gut contraction matches that of both square cell disappearance and both the long-term disappearance of granular ingested material and the production of the micro-granular refractive egestive material - but not that of lumen disappearance-points to cellular 'degeneration' being the major determinant of overall gut size decrease. The process's 'echo' from Bowman (2017a) is found!

Evidence for a second physiological 'echo' or possible 'slower' intracellular digestive phase on 'tougher' prey material is shown posteriorly in Fig. 13, lower-see the anticlockwise extra loops. This is driven by: a surplus of small vacuolation late on-Fig. 17, lower and the Fig. 18, middle row second shaded 'pyramid'; and, a slow re-equilibriation of the gut epidermis to the starved state both in terms of the temporality of gut lumenation and of lumenal refractive grain appearance (Fig. 20, lower-final decline). This could simply be a function of the majority of the ingested prey food being taken into the larger volume posterior part of the midgut at a later time point in imbibition as the prey is progressively dissolved extra-corporeally. However, clearly late-on imbibed material is likely per force to be more resistant to extra-corporeal digestion - and thus possibly intracellular digestion too. Bowman (2017b) already found evidence for the differential lability of prey fractions. Five half-lives of gut lumenation (cf. Table 1) from the start of cell degeneration (Fig. 20, lower) i.e. to its likely completion, is around 2 days from the start of feeding. This agrees with the zenith of gut lumenation and when the egestive phase is well underway (cf. second rise in dashed line in Fig. 18, middle row). This indicates indeed that the first shaded 'pyramid' of small vacuoles (in Fig. 18, middle row), and the large vacuole 


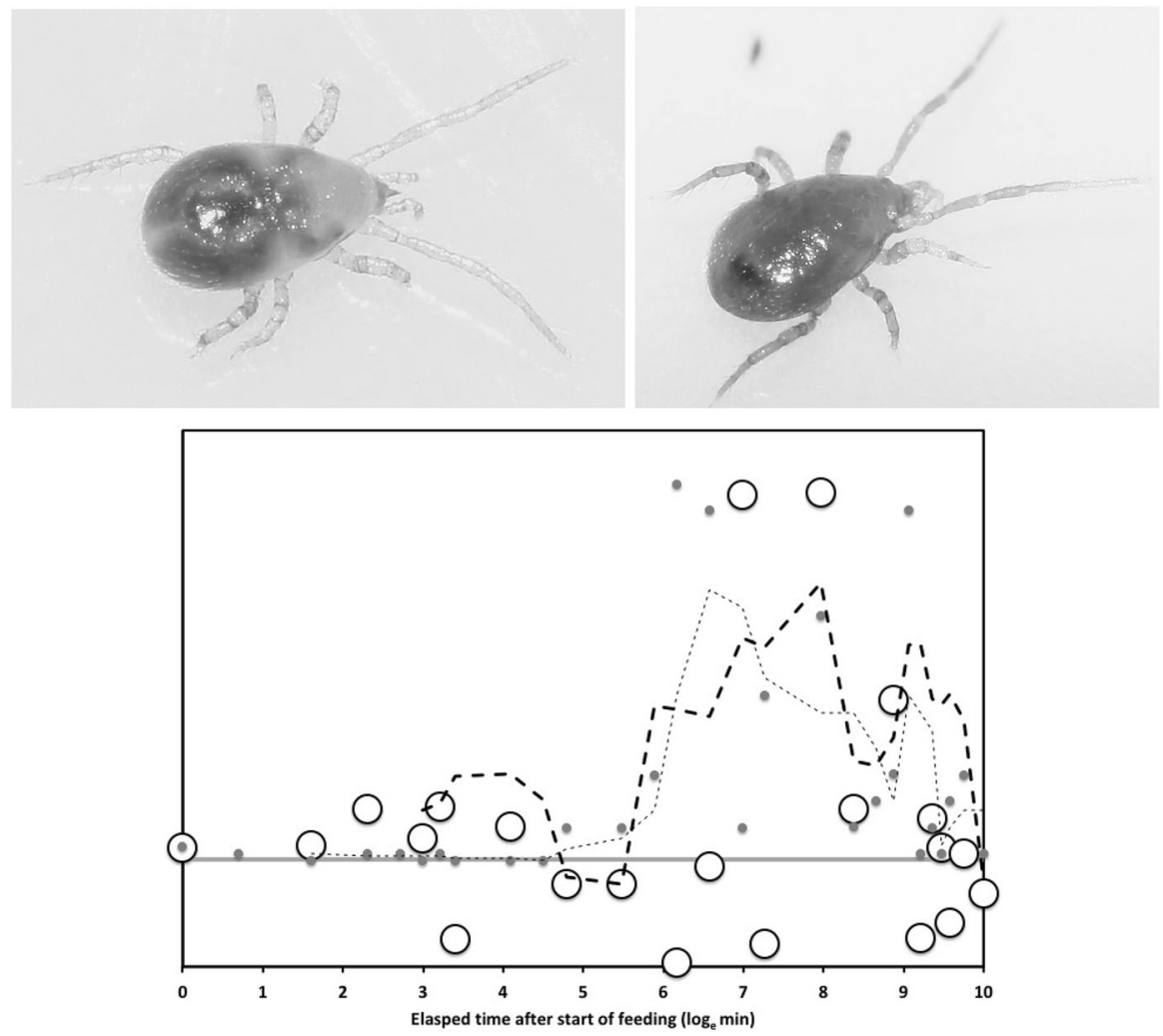

Fig. 20 Egestion in Pergamasus longicornis. Upper: pergamasids showing dark egestive refractive grain material. From colour photographs: In Laub/an Totholz OTobias Töpfer (29th February 2012) with permission. Left nymph showing dark presence throughout mid-gut—matches $\log _{e}$ values of $6.5-8$ ( $\equiv 12 \mathrm{~h}-2$ days after start of feeding in Bowman 2017b). Right. Mite showing dark presence only in rectal vesicle-(nothing anywhere in the mid-gut now-so this must be egestive material only). Note the faint beginnings of Malpighian tubules glistening under the idiosomal integument either side. Matches $\log _{e}$ values of 8.9-9.3 (इ5-8 days after start of feeding in Bowman 2017b). Lower: cellular disintegration matches pattern of (dark) egestion. Ratio of lumen standardized score (from Bowman 2017b) to expansion-contraction standardized measure from Bowman (2014) = 'lumenation' on $\log _{e}$ timescale after the start of feeding on larval dipteran prey. Large open circles are observed ratio. Grey line is overall average ratio (baseline). Dashed black line is sixth order moving average trend for ratio. Small grey circles and pale dotted third order moving average trend line are probability of refractive grains (from Bowman 2017b). Note fitted lines for the steep rising change in lumenation indicating disproportional cell loss (and then the falling re-equilibriation of relative surface area) correlate well with refractive egestive grains appearing (and disappearing) during the later phases of digestion. Estimated half-life of lumenation: rise over $\ln (\min )$ values of 4.8-7.0 (E2-18 $\mathrm{h}$ period $)=7.7 \mathrm{~h}$; fall over $\ln (\mathrm{min})$ values of $8.0-9.5(\equiv 2-9$ days period $)=4.6$ days

peak at 6-8 h (Fig. 17, lower) are likely to be a focus of fast early absorption and digestion of the easy-to-catabolise ingested prey material giving the first rapid rise in egestion/ egestive release. Whilst the second shaded 'pyramid' of small vacuoles (in Fig. 18, middle row) is the longer slower fasting response around the intracellular breakdown of potentially tougher prey material and the long tail-out of refractive grain production (Bowman 2017b) before starvation sets in. In that way, the pergamasid midgut epithelium may be quickly ready physiologically for the mite to potentially consume another victim in short order (a 
Fig. 21 Prey food processing in Pergamasus longicornis. Intracellular food inflated by $150 \%$ for ease of display. Top row: overall schema on $\log _{e}$ time scale (in min) after commencement of feeding on dipteran larva. Upper Malpighian tubule annotation and digestive phase vertical break point lines as in Bowman (2014, 2017a). Large horizontal grey dashed arrow indicates ' $O$ ' = osmoregulatory (coxal droplet) phase (Fig. 13). Small black points and sixth order polynomial dotted trend line $=\frac{1}{\text { lumenation }}$. Large grey circles and second order moving average solid trend line = extracellular food. Large open circles and dashed second order moving average trend line = intracellular food. Grey triangles and lower grey spotted second order moving average trend line $=$ extracellular waste. Note parallel catabolic fall in epidermal predominance, intracellular food, and extracellular food from about $8 \mathrm{~h}\left(\log _{e}\right.$ time $=6$ after the start of feeding) indicating rate-limiting ingestion/absorption. Second row centrally: Left: hysteresis between intracellular and extracellular food. Anti-clockwise rise before approximately $8 \mathrm{~h}$; and, fall after approximately $8 \mathrm{~h}$ from the start of feeding. Right: hysteresis between lumen and cells. Anti-clockwise rise as 'thin' cells induced by ingestion forcing gut expansion; and, then fall as they transform to 'square' cells. Grey circles and marbled lefthand arrow shows period of Malpighian tubule expansion with guanine; then contraction as excreta shipped into the rectal vesicle. Lower third and fourth rows: nutritive staging. Large arrows indicate flows. (1) 'I' and 'O' = Ingested prey food arrives [and is also concentrated through fluid recycling-Bowman (2014)]. (2) 'O' and ' $\mathrm{D}$ ' = Prey food digested extracellularly/corporally is absorbed into the ever expanding and growing gut epidermis wherein it begins intracellular digestion. (3) 'D' = Intracellular digestion increases apace even as extracellular food is exhausted. (4) ' $\mathrm{E}$ ' = egestive residues from intracellular processing are then shipped out as extracellular waste (just as opisthosomal protein content rises-see Fig. 19, lower)

few days or so on from the first), but before the first meal has been completely processed. Tracking immuno-histochemically the different sorts of small vacuoles and their prey contents in future work would confirm or refute this.

Whether the mechanism of gut cell degeneration is by activation of autophagy-dependent cell death (Moura-Martiniano et al. 2017) remains to be confirmed. However, Fig. 21 illustrates lumen occurrence together with the concentrative osmoregulatory and excretion phases from Bowman (2014, 2017a) to contextualise this conclusion. Gut lumen fluid specific gravity and osmotic pressure would be expected to increase at this stage as in argasids (Hefnawy 1972b; Hefnawy and Dimos 1974). Note the parallel rises of extracellular and intracellular food. Also, the parallel fall in epidermal predominance, intracellular food and extracellular food from about $8 \mathrm{~h}[\ln ($ time $)=6]$ after the start of feeding indicating rate-limiting initial ingestion/absorption. An 'egestive switch' ('Check (c)') does appear to be occurring after $4 \mathrm{~h}(\ln ($ time $)=5.48$ in Fig. 20) just as lumenal changes inferred. This marked rise in 'lumenation' of the gut through to $48 \mathrm{~h}$ [ $\ln ($ time $)=7.97$ ] is exactly around the time where the results in Bowman (2017b) point to digestion of the primary prey material coming to a conclusion (i.e., 18-34 h) and the major rise in lumenal refractive grains. Moreover the estimated half life of the rise in lumenation $=7.7 \mathrm{~h}$ and that for the fall $=4.6$ days (Table 1) is very close to the corresponding half-lives for refractive grains appearance and their disappearance in Bowman (2017b). This is strong corroborative evidence for the earlier observations by other authors of epithelial cells observed in sections to be degenerating eccrinally or apocrinally (Šustr and Hubert 1999; Filimonova 2009). Quantitation of the burst in gut cellular relative surface area supports previous histological conjecture! Follow-up work should check if these degenerative cells look cytologically like those in B in Fig. 3 of Starovir (1973) and like those of Fig. 5 in Starovir (1982). More detailed TEM data is also needed to check if this process first might favour the anterior gut regions then the posterior regions - as needed for Bowman (2017b)'s view on the different scheduling of refractive grain production. However, for sure, the mismatch temporally between this cellular degeneration (aka 'lumenation') and the need for the (earlier) extracellular digestion (if true) of ingested prey soon on after ingestion suggests that released cellular debris are not likely to be a major source of such soluble enzymes as suggested by Reichenow (1918). 

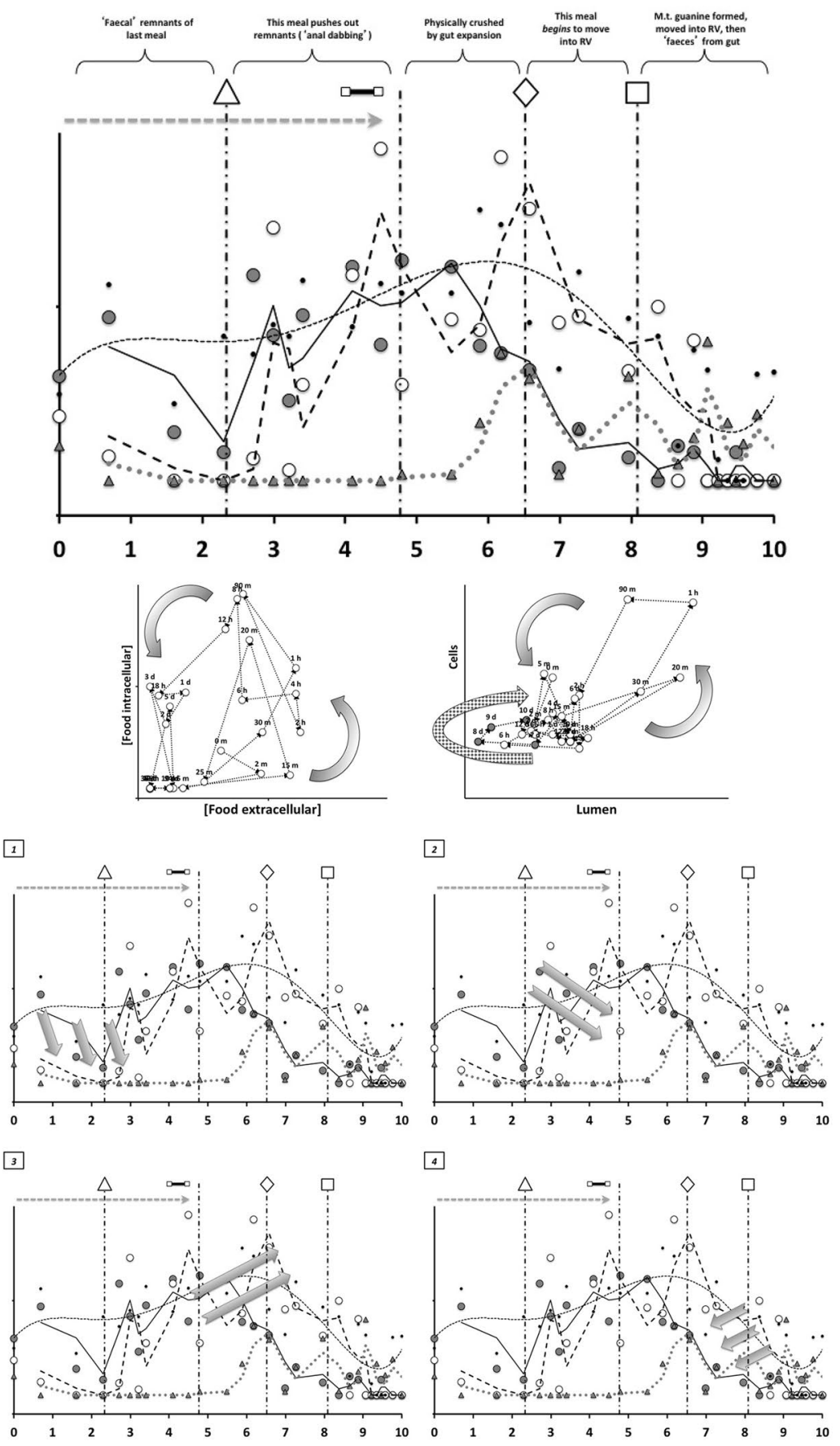

The commonality of 'stiffness' in various sub-systems suggests that the simple system model in Bowman (2017a) is supported (estimates of effective volumes of distribution $\left(\frac{D}{V}\right)$ in the various explicit compartmental equations could be made if required). Figure 18 
shows how the detailed results of lumenation and vacuolation can be integrated into this scheme. The mid-gut (and endodermic hind-gut) in P. longicornis appears to be behaving like a single stomach/intestine and like a single liver-transporting material and swelling on feeding, being called upon for resources during fasting and shrinking. It swops from digestion-predominating to catabolic egestion-predominating in a smooth manner over time. If gut epithelial degeneration did sweep posteriorly over time, it would both fit with the anterior regions being FIFO (for size and prey contents-Bowman 2014, 2017b), and fit with the indications (Bowman 2017a) that posterior parts of the Malpighian tubules have a greater occurrence of guanine in them than their anterior parts - the same catabolic wave thus accumulating pygidially. Note that no sorting of prey material occurs. The whole physiology (i.e., feeding, digesting, egesting and excreting system) of P. longicornis appears to be behaving like an integrated single conveyor belt.

\section{FIFO, LIFO, LILO and the total feeding cycle time}

Cell degeneration has already been predicted as the likely cause of gut changes from previous work (Bowman 2017b). In this study, cellular catabolism in general sweeps pygidially-but jigsaw-like cell vacuolation is still present late on anteriorly. So, this study thus confirms again his hypothesis (iv) (i.e., 'Check (d)' in the "Review of the Field"). Previous work has pointed out that based upon overall size changes the anterior pergamasid gut behaves like a LIFO ('push-pop-stack') process, and the posterior part behaves like a FIFO ('conveyor belt') process (Bowman 2014). However, based upon the appearance and disappearance of lumenal granular material, the anterior part of the mid-gut appears as a 'deep storage' LILO process, the posterior a FIFO process (Bowman 2017b). So which is it?

Although Repkina (1976) did not find a strict link between zones of different degrees of physiological ageing stages and a particular part of the tick mid-gut, Akimov and Starovir (1977) points to the central parts of the mesostigmatid gut being 5-15 min earlier in their histological changes than the rest of the gut. This is not supported for the mesenteron in $P$. longicornis-see Figs. 4, 5 and 6. However, as Lagutenko (1963) points out that $\frac{2}{3}$ rds of the mesostigmatid opisthosoma is filled with the gut, and the anterior caeca are so small in proportion to the posterior volume (Bowman 1984a), then this central to non-central comparison is essentially the same contrast as between the posterior regions versus the rest. So, what is known in other mites?

Starovir (1973) notes functional differences between the cells of the central part of the mid-gut and those at the distal ends of the diverticula in P. persimilis. Diagrams in Kanungo (1964) suggest that in E.echidninus, anterior and posterior parts of the mid-gut clear of engorgement together-although the apparent extent looks greater posteriorly due to the larger caecal sizes. Bowman (2014) reports that the anterior parts of the gut in $P$. longicornis form a 'conveyor-belt' process - a claim critically assessed by Bowman (2017b). If one fits separate models (over 0-90 $\mathrm{min}$ ) for the appearance of vacuoles this yields half-life estimates of $1.2 \mathrm{~h}$ versus $1.1 \mathrm{~h}$ for the anterior versus the posterior mid- and hind-gut regions. As the global log odds ratio test suggests, this is not significantly different from each other. So, whilst gut size may swell and shrink anteriorly first (together with a lumen appearing first anteriorly-Bowman 2017b), there is no evidence for the anterior regions having FIFO vacuolation (or a markedly speedier lumenal disappearance-Bowman 2017b). The anterior regions may expand first and contract first, filling early and first with a speedy lumen appearance but they empty slower-yet first-due to their smaller size (and probably lose their lumen here first too for the same reason). This expansion/ 
contraction or filling/emptying of the anterior regions is not by a difference in vacuolation or devacuolation rates. Indeed, fitting separate processes for vacuole disappearance, yields half-lives of 1.4 days versus 1.1 days for anterior versus posterior regions (over the $8 \mathrm{~h}-6$ days time window) and 2.4 days versus 1.9 days respectively over the full 8-366 h window. These pairs of rates are broadly consilient. Food elimination is actually slower in these facultatively 'expendable' anterior gut regions compared to the posterior parts (i.e., the antithesis of hypothesis (iv) in Bowman 2017b). This apparently inconsistent or converse behaviour could be due to the posterior parts of the gut having a greater involvement in holocrine secretions (and the cause of globular lumenal contents perhaps?) or a greater role in refractive grain production relative to the anterior parts (see discussion above). There is therefore only some evidence from mid- and endodermal hind-gut cell vacuolation that posteriorly the gut does behave as a LIFO process which Bowman (2014) claimed. The anterior midgut in $P$. longicornis is more like a mixture of FIFO and FILO. However it does not (Fig. 12) appear that the anterior part preferentially produces possible extracellular enzyme-carrying free cells.

Concluding that the posterior regions of the midgut in $P$. longicornis are LILO compared to the anterior regions suggests that if there is a 'slow intracellular digestion' phaseacting upon the most catabolically tough prey imbibed material-this is where it would mainly occur (if only by volume). As discussed above, it would be consilient with a possible second physiological 'echo' shown by Fig. 13, lower. Here late on, there is a surplus of small vacuolation-Fig. 17, lower and Fig. 18, middle row; and, a slow shrinking re-equilibriation of the gut epidermis by the loss of gut lumenation (increase in relative surface area) back to the starved state (Fig. 20, lower, $t_{\frac{1}{2}}=4.6$ days-Table 1). Alternatively, this double peak of vacuoles could simply be the relative contributions of the FI anterior regions (appearing 'fast') versus the LILO posterior regions (appearing 'slow') to the overall picture. Further work is needed.

In ticks Repkina (1976) draws attention to earlier work from Knyazeva (and to Razumova 1977) showing the uneven consumption of reserve nutrients in different sections of the tick mid-gut-noting a brisker turnover in the anterior processes compared to those posteriorly. In $P$. longicornis it is likely that despite the rate of vacuole elimination being faster posteriorly, food depot stores persist there posteriorly in the idiosoma simply by virtue of the large size of the posterior caeca and their visual dominance in the idiosomal volume. Facultatively therefore-although comprised of the same fundamental cell type and a single tissue - the posterior mid- and endodermic hind-gut epithelium actually behaves like a LILO (last-in-last-out) deep storage tissue.

Arachnids have been known for a very long time to store fat and glycogen in the mid-gut (Blanchard 1855a; Plateau 1877; Bertkau 1885; Guiyesse 1908). The cells of the midgut in ticks serve as a depot of stored protein, fats and carbohydrates, realising a complicated chain of metabolic conversions-Balashov (1961b). Fat bodies for nutrient storage are also known in ticks (Obenchain and Oliver 1973) and link feeding to reproduction (Coons et al. 1990). Fat bodies are the site of intermediate metabolism as well as a place for storing nutrients (El Shoura 1989) in argasids. In some sense therefore the caeca of posterior midgut in P. longicornis are like a (non-fat dominated) 'fat body' physiologically. Whether there is a vitellinogenic fat body trophocyte-like acidophilic cell in the mid-gut of pergamasids like in tick guts (Rosell-Davis and Coons 1989) or true fat bodies and associated tissues (Obenchain and Oliver 1973) remains to be seen. Are there peripheral basophilic nephrocytes in $P$. longicornis also involved with carbohydrate and lipid storage? It is of note that the pergamasid mid- and hind-gut epidermis itself does retain some (albeit 
reduced) whole system characteristics in this way-unlike the Malpighian tubule excretory guanine system that showed neither LIFO, nor FIFO processes (Bowman 2017a). This fact confirms that the gut histological dynamics are upstream of the excretion processes.

Bowman (2017b) questioned if the earlier use of gut expansion/contraction measurements may have slightly underestimated the contribution of egestion to the total feeding cycle time. Inspection of gut cells microhistologically confirms that this is so-cells are still showing vacuoles ( $a k a$ intracellular digestion); and, also indirect evidence (via the lumenal proportion of the gut) of continuing egestive release through to about 6 days after the commencement of feeding. This is a little longer than the time for the loss of black egestive pigment (haematin?) on starvation in ereynetids (3-4 days, Baker 1971), but is now more consistent with the complete disappearance of the blood meal in Echinolaelaps echidninus of 5-7 days (Kanungo 1964) and the estimates of the biological half-life of ingested material in cryptozoans ranging from 5.5 to 27 days by Reichle and Crossley (1965). The total feeding cycle time allowing for the majority of intracellular digestion and cellular (egestive) decline is much more like the one week originally claimed without substantiation by Bowman (1984a). Comparison to the results of Bowman (2017a) suggests that excretion of the single prey meal may take a subsequent 3 days to typically run its course. Final complete clearance ['Check (a)'] may take much longer ( $>15$ daysBowman 2017a). Starvation only occurs after lengthy fasting in $P$. longicornis and is best described by the lack of cellular vacuoles in shrunken alumenate gut caeca ('Check (f)'). As in Calyptostomids (Vistorin-Theis 1977a), pergamasids are adapted to be able to starve for a long time and may not eat often in the wild.

\section{Assimilation efficiency}

Despite their voracity, gamasines overall only ingest moderate amounts of prey dry weight per day (0.8-1.3\%dry wt body equivalents/day-McBrayer and Reichle 1971). However, is this well used? In animals, assimilation is defined as [ingestion-egestion] and assimilation efficiency as $A E=\left[\frac{\text { assimilation }}{\text { ingestion }}\right]$. Is there evidence that ingested food does actually yield body tissues in pergamasids?

Figure 19, lower panel shows the protein content (as $\mu \mathrm{g}$ BSA equivalents) from Bowman (1985) over both genders categorised for each observed nutritive state in the opisthosoma (i.e., [-:-], [dark:-], [-:white], [dark:white]) of the 101 studied pergamasids at collection from the wild. Protein content values are displayed over feeding to fasting for the period of the maximum posterior probability of that state (estimated from-Fig. 21 in Bowman $2017 \mathrm{a})$. The overall body protein content rises over $1-10$ days $(\ln ($ time $)=7.27-9.57)$ from the start of feeding with a distinct peak around 9 days $[\ln ($ time $)=9.47]$ before a dramatic fall. This shows that it is neither the primary imbibed food nor the main gut epidermal growth (see Figs. 7, 8, 9) nor the vacuolation (see Fig. 11) being assayed here. Rather the measured protein appears to be an accumulated 'metabolic pulse' contingent upon the consequence of the second wave of small vacuoles (during the egestion phase-see lower panel in Fig. 17 and middle panel in Fig. 18) feeding through to body tissue formation (and final nitrogenous waste excretion). The drop thereafter is the onset of fasting and then starvation. The possible 'slow' or 'tough' prey material digestive phase may thus be related to body protein synthesis by the pergamasid. This needs tracking metabolically.

Can an estimate of assimilation efficiency judge the predatory effectivity of $P$. longicornis? Using Bowman (2017b) and summation over regions at each time point: the concentration of extracellular food at time $t$ was estimated as: 


\section{$\frac{\sum[\text { granular }+ \text { globular }]}{2 \sum[\text { lumen }]}$}

scores for each time point. The concentration of intracellular food at time $t$ was estimated as:

$$
\frac{\sum\left[\text { thin }_{\text {sml }}+\text { thin }_{\text {lge }}+\text { square }_{\text {sml }}+\text { square }_{\text {lge }}+\text { jigsaw }_{\text {sml }}+\text { jigsaw }_{\text {lge }}\right]}{6 \sum[\text { thin }+ \text { square }+ \text { jigsaw }]},
$$

scores for each time point (where the subscripts are $\mathrm{sml}=$ small, lge $=$ large, vacuoles). The concentration of extracellular waste material at time $t$ was estimated as:

$$
\frac{\sum[\text { refractive }+ \text { membranous }]}{2 \sum[\text { lumen }]},
$$

scores at each time point.

Total ingestion ( $\equiv$ one dipteran larva) can be estimated for P. longicornis from Bowman (2017b) by the AUC of extracellular granular lumen material ( = 1004.9 units). The scale of total egestion is indicated by the AUC of extracellular egestive grains in the gut lumen ( $=2808.6$ units). However, the latter first needs to be converted to common physiological units with that of ingestion. This can be done via the vacuolar measurements as an intermediate physiological scaling as follows. Histologically prey food is assimilated via the gut epidermal vacuoles described above (whose AUC = 2930.1 units). Given complete absorption into vacuoles, (in $P$. longicornis, there are negligible indigestible ingested residues in the lumen-see 'Membraneous and other' in Bowman 2017b) each ingestion unit therefore is represented by $\frac{2930.1}{1004.9}=2.92$ vacuolar units. Equivalently therefore ingesting one dipteran larva generates 2930.1 vacuolar units of absorbed ingestion. Now turning to the rescaling of egestion physiologically. Vacuoles appear histologically related to egestive grains-vacuolar cells being inversely related to lumenation (see above). The ratio of total egestion to total vacuolation is $\frac{2808.6}{2930.1}=0.95$. So on the face of it each egestion unit could be represented as 1.04 vacuolar units. However, the micro-crystalline refractive egestive grains are formed from absorbed material by a parallel catabolic process to that of guanine production in Malpighian tubules (Bowman 2017a, b) whose stoichometry is estimated as driven by a 5.5 factor (Bowman 2017a). So the assimilation efficiency assuming no respiration and this stoichometry $=1-\frac{(2808.6 * 1.04)}{(5.5 * 2930.1)}=0.82$ for $P$. longicornis. This agrees well with the known percentage assimilation efficiencies of insectivores of between $70 \%$ and $80 \%$ and that of carnivores at $90 \%$. Predatory araneids have assimilation efficiencies of $89-100 \%-$ higher than that of terrestrial predatory insects (58-84\%, Wiegert and Petersen 1983), so this pergamasid is physiologically typical of arachnids. Food conversion into pergamasid tissues will be lower than spiders because of large respiratory losses in maintaining an active predatory lifestyle — recall the 10\% law of trophic transfer (Lindeman 1942).

\section{Looking to the future}

The cell types suggested in the past within the digestive tracts of arachnids are quite diverse (even between mites and ticks). As this review recounts, it has not been clear if these distinctions are apparent or real. This paper provides evidence that, for at least $P$. longicornis, 
there is only one type of cell fundamentally that varies along the course of digestion and egestion carrying out the bulk of the gut physiology. However, only the exhaustive association of image and molecular characterization including of specific cell markers will unequivocally clarify this.

Even in the much larger chelicerates the ultracytochemistry of the gut is hardly ever studied-Warburg (2012). Other enzymes could be tracked histochemically. For instance succinate dehydrogenase as a marker for mitochondria as already done in ticks-Weber (1974). Detailed TEM and molecular data are needed comparatively across the whole Arachnida - the recently deceased acarologist Gerd Alberti (University of Greifswald) has shown the way in his many publications. The dynamical changes in cells' inclusions need to be followed and correlated experimentally with physiological processes-Warburg (2012). Perhaps this could be linked with immunocytochemistry of the breakdown of prey antigens (like erythrocyte digestion is tracked in ticks-Osterhoff and Gothe 1966)? Mass spectrometry imaging over time would allow molecular and structural comparisons along the digestive tract with the enzymes and other proteins involved in food processing in $P$. longicornis. Analysing quantitative TEM characters under experimental conditions kinetically over time would be very illuminating.

Despite widespread use in other cellular fields, technologies relying upon fluorescence have only been rarely used in mite studies-Occhipinti and Maffei (2013). Although the cuticle of $P$. longicornis is more sclerotised than tetranychid mites-given that soil predatory mites must be consuming plant material from the guts of their herbivorous preytracking the fate of chlorophyll degradation products could be useful in understanding the kinetics of gut processes in free-living mesostigmatids (that had been fed tetranychids experimentally for instance). For sure, confirmation of exactly what the egestive and excretory products might contain (not just guanine) by enzyme digestion and fluorescence microscopy would clarify the proposals in Bowman (2017a). Unlike ticks (Hamdy 1977), do non-blood sucking mesostigmatids actually completely degrade ingested protein and do not excrete any protein? As top predators (for their size) in the forest floor, P. longicornis must concentrate and accumulate toxic products of their prey (and plant secondary metabolites from eating herbivores). Where are these sequestered? How are they processed and eliminated? Is nutritive status in mesostigmatids linked to reproduction as in ticks (Kitaoka 1961c; Balashov 1962; Aeschlimann and Grandjean 1973) or is there no gonotrophic relation as in Haemaphysalis casalis-Men (1959)? Could radioactive tracer studies (like Rodriguez and Hampton 1966) inform what is going on metabolically in mesostigmatids? Could tracer experiments test if mesostigmatids regurgitate like other arachnids-Kullmann and Kloft (1968)?

This study has not looked for evidence of diurnal cycles in gut physiology (by experiments where the start of feeding is at different planned times of the day). Also, glycogen is high in ticks (Wyss-Huber von et al. 1961) and glucose and glycogen levels vary diurnally in scorpions-Raju et al. (1973). It would be interesting to look for such in histochemically or biochemically measured gut characteristics in P. longicornis-perhaps this is the reason for the rhythmical 'wave' in the total profile in Fig. 19, upper? Similarly the effect of predrinking water (Blommers et al. 1977) has not been investigated.

With the advent of Next Generation Sequencing (NGS) and other high-throughput bioinformatic-based technologies, transcriptomics (mRNA) and proteomics can be carried out in pulse-chase feeding experiments. This has allowed Fuzita et al. (2016a) to elucidate the gut physiology of the scorpion Tityus serrulatus (where the midgut and midgut glands are composed of both secretory and digestive cells). In fasting animals, the secretory granules are ready for the next predation event, and contain enzymes needed for alkaline extra-oral 
digestion such as trypsins, astacins and chitinase. This material also comprises the refluxing digestive fluid. There is no clear evidence that $P$. longicornis regurgitates, but does its salivary glands contain alkaline proteases? The digestive vacuoles of scorpions are filled with an acidic proteolytic cocktail for intracellular digestion composed of cathepsins L, B, F, D and legumain. Other proteins as lipases, carbohydrases, ctenitoxins and a chitolectin with a perithrophin domain were also detected in scorpions. Is the production of these switched on in mesostigmatid feeding? Proteolytic activity in ixodids appears to be related to acidic proteases Bogin and Hadani (1973). Verifying if, in free-living predatory mites, there is increased transcription of sets of genes related to known gene ontology (GO) defined functional pathways (like the autophagy pathway in ticks-Moura-Martiniano et al. 2017) could be useful. Is this latter pathway the basis of the gut epidermal egestive mechanism (Bowman 2017b)? What could NGS dynamics say about the mucopolysaccharide mediated excretion processes reported in acarines-Khalil 1971; Balashov and Raikhel 1973, 1976b? Could transcripts for enzymes to form hypoxanthine, or for xanthine dehydrogenase clarify how close mesostigmatid excretion is to other arachnids (Anderson 1966) and uric acid excreting insects?

Using such technology to track whole genome responses in chelicerates is very powerful-Bensoussan et al. (2016). A recent detailed application of this high-throughput approach in a spider (Fuzita et al. 2016b) has shown a rapid (within $1 \mathrm{~h}$ of commencement of feeding) up-regulation of genes related to the: feeding stimulus; diet hydrolysis (especially the astacin class of digestive enzymes); and, vesicular trafficking. Lack of up-regulated proteins and evidence of protein down-regulation was found $9 \mathrm{~h}$ after feeding. Differential location of assays between digestive fluid and mid-gut diverticula fitted a rational scheme covering proteolysis, digestion and extracellular secretion. Interestingly expression patterns for intracellular digestion were not found to be markedly different from fasting and fed animals which matches the observation (Ludwig and Alberti 1988b) that arachnid digestive vacuoles remain histologically intact for long periods (cf. persistence in Fig. 17, lower).

Astacin gene duplication appears to be a phylogenetic signal for the need to liquefy the food externally in arachnids - it would be very interesting to assay free-living predatory mesostigmatids like $P$. longicornis to see which clade they cluster with (and whether astacin up-regulation matches the occurrence of 'free cells' with 'bead-lets' in early digestion?). When might such 'bead-let carrying' cells be synthesised? Do transcripts disappear in synchronisation with the cytological results? Perhaps astacins could be shown in mesostigmatid salivary glands? Given the partial commonality of protein sequences between venoms and digestive fluids in spiders (Walter et al. 2017), is immunohistologically tracking particular protease transcripts the way to prove that mesostigmatids inject a salivary 'toxin' into their prey to subdue it? For sure the evolution of extra-oral digestion in spiders and their feeding strategies is tightly linked with the evolution of silk and venom useextra-oral digestion being in particular regarded as an expression of an extended phenotype. Could evidence be found as to how mesostigmatids might produce and utilise silk too? Could anticoagulant abilities (as in ticks-Nuttal and Strickland 1908; Nuttal 1909; Markwardt and Landmann 1961) be detected in this way? The use of enzymes outside the protective milieu of the mites' body requires the incorporation of assisting agents that regulate the activity and decelerate the degradation of active components in the open or in the unpredictable environment inside the prey's body - Walter et al. (2017). Enzymes in digestive fluids need to be very potent as only very small amounts are produced and released by any arachnid to quickly dissolve a potentially large amount of prey tissues-speed up or facilitation by 'helper proteins'/cofactors will be very important. Do transcripts show 
evidence for these specialist moieties (like hyaluronidases, elastases, collagenases) in $P$. longicornis? What can NGS say about the evolution of mite and other arachnid pre-oral digestion (Grasshoff 1978; Weygoldt 1998)?

Perhaps among the genome sequences for chitinases that might be found in mesostigmatids, a chitolectin (chitinase 3 ) with a peritrophin domain which is catalytically inactive might be found - as is reported in a scorpion (Fuzita et al. 2016a)? Or a transcript for 'Der P23' material (Weghofer et al. 2013) found in mesostigmatids? This might resolve the confusing reports in mesostigmatids of a peritrophic membrane being observed in some species (Wharton and Brody 1972; Rudsinska et al. 1982; Zhu et al. 1991; Sobotnik et al. 2008b) and not in others. Could there be the presence of a peritrophic gel produced even in fluid feeders? Is this the lumenal membranous material scored in Fig. 6 of Bowman (2017b) late on after feeding especially in the rectal vesicle of $P$. longicornis? Does a membraneous gel encapsulate the refractive grains 'dust'?

Choice of 'shot-gun' technology will be important. Use of liquid chromatography and tandem mass spectrometry analysis (LC/MS-MS) of the mid-gut proteome of O. moubata females failed to find great differences in the number or class of proteins expressed, enzymatic composition or functional classification before and after blood feeding-Oleaga et al. (2017). This was despite a detailed analysis of particular groups of proteins that are involved in the processes of nutrient digestion and responses to the oxidative stress being carried out. The genes and proteins involved in intracellular transport mechanisms, defensive responses, detoxifying responses and stress responses seemed to be closely regulated-an expectation for any tightly integrated body system.

It might be possible to unpick some of the pergamasid digestive cells' endocytotic mechanisms using qPCR-enabled expression profiling. Legumains along with many other types of digestive proteases have been shown in spider mites-Santamaría et al. (2015b). Legumains are usually associated with endopeptidolytic activity in lysosomes involved in vacuolar processing, maturation of receptors and turnover of the extracellular matrix (Hartmann et al. 2018). Parasites such as platyhelminths, nematodes and ticks have been shown to express legumains as important members of multi-enzyme complexes that perform proteolytic degradation of host blood proteins. As in P. longicornis it seems that, like in ticks (Sojka et al. 2013), the gut lumen serves as food storage space and digestion occurs (almost) exclusively in vacuolar vesicles of the gut cells-use of RNA-seq and expressed sequence tag (EST) data-sets in inhibitor-treated mesostigmatids might shed more light as to what is going on in the gut epidermis physiologically in detail.

Whatever future methodology is chosen, one advantage of these high-throughput technologies is that they could also be modelled kinetically themselves and thus aligned not just with the tissue time course but also with the dynamics of traditional histological and biochemical enzyme assays across mite ingestion, digestion, egestion and fasting. For sure, comparing in this way re-estimated values from previous argasid tick researchers (Table 7) to the half-lives for granular lumenal material, refractive grains or Malpighian tubule guanine appearance/disappearance as appropriate in Bowman (2017a, b) suggests that all anactinotrichid acarines may have the same fundamental physiological characteristics for their digestive and excretory tissues. Are actinotrichid mites different? What of other arachnids (Sinha and Kanungo 1967, etc), one wonders which clades are physiologically the same as mesostigmatids? Many zoologists may be sitting upon a 'gold-mine' of physiological data in laboratory repositories just waiting to be re-analysed kinetically (e.g. Aeschlimann and 
Grandjean 1973; O'Hagan 1974 etc) and to inform comparative arachnology (Lindquist 1984; van der Hammen 1989; Talarico et al. 2011). Let's find out!

\section{Conclusion}

As in ticks (Tatchell 1964), the feeding and digestive processes of $P$. longicornis are such as to enable the animal to ingest in a short time the maximum amount of food it can at one opportunity-retaining a portion of this meal as a long-term slowly processed food reserve. This gut epithelium-based transformation obviates the major need for a fat-body as in insects (Kilby 1963), or an interstitial tissue store as in scorpions (Goyffon and Martoja 1983), or a discrete 'liver' as in other chelicerates. Tissue level differentiation rather than organ level differentiation marks out the physiology of pergamasids. So, what can be concluded about this simple enteron (Leake 1975) for specific verification in other mesostigmatids?

Although the rectal vesicle is a distinct ectodermic storage vessel, the endodermic midand hind-gut in P. longicornis behaves like a single epithelium (Fig. 20), as in phalangids-Phillipson (1961), or scorpions-Goyffon and Martoja (1983). However, it shows three distinct forms: squamous; columnar; pseudo-stratified; all in various proportions as the gut expands and contracts over time. This is in line with the ultrastructural observations of El Shoura (1988) where apparently discrete gut cell types interconvert, reactivate and transform during argasid digestion. In fact, the pergamasid gut epithelium undergoes changes in size and form all the time-so when sections are examined at one particular nutritive stage, the epithelium appears different from that of another stage. This difference in appearance could be easily mistaken as evidence for different cell type (as Roesler 1934 claimed for ticks). However, the consilience of the kinetics and the temporality of food-processing phenomena show that this is an illusion. Gradual continual conversion is the case. If pergamasid digestion was examined after sequential ab libitum prey feeding then these cellular changes would all overlap in a complicated histological picture needing experts (Akimov and Starovir 1977; Starovir 1979b, 1981b) to discern the distinct physiological processes going on.

As in tick guts (Franta et al. 2010), intracellular processes are the main cell activity in this executive organ. The digestive-absorptive cells of the mid-gut can and do carry out the entire sequence of post-oral food processing, absorption and egestion-the cellular occurrences over time being summarised in Figs. 17 and 22. There appears to be fundamentally a single polyvalent type of gut cell, like: the only one type of epithelial cell in the ventriculus and the caeca of the prostigmatid Calyptostoma velutinus (Vistorin-Theis 1977b); the single digest cells of ixodid ticks (Agyei et al. 1992, i.e., those in the first continuous digestion phase-Tarnowski and Coons 1989); the multifunctional cells of amblyseiids (Akimov and Starovir 1977); and, the arthropod digestive cell type sometimes called 'B-cell'. The single cell type can take different physiological states as in S.fringilla (Filimonova 2009) and as in ticks (Coons et al. 1986; Agyei and Runham 1995). Whether it is or is not also the 'bead-let' carrying free floating cells observed awaits unequivocal confirmation.

Like in P. persimilis (Akimov and Starovir 1974), the functional condition of the epithelial cells depends upon the degree to which the mite's intestines are filled with food. More lumenal food suggests (at least early on in imbibition) more potential contact surface with epithelial cells. So as Mitchell (1970) says, the many mid-gut 'lobes' or caeca may be devices to increase any possible gut epithelial cell surface area limit to processing 
(clavate cells obviate this). Starovir (1979a) points to the mid-gut diverticular folds being a (further) predatory adaptation for absorbing food in quantity. The act of mid-gut expansion creates an increased surface area for digestive processes and reduces the distance of the mid-gut and caeca from the internal organs that depend upon nutrient transport from the gut (Pritchard et al. 2015). The extensibility of the gut cells facilitates the uptake of a considerable amount of food (as in A. casalis-Starovir 1980). Future work should include measuring gut cell lumenal surface and total cell volume directly to check this from feeding to fasting.

Early on in digestion the most common tissue types are either squamous or cuboidal/ columnar depending upon the degree of gut distension (much as in P. ovis-Mathieson and Lehane 2002). Squamous and cuboidal cells are the common ventricular and caecal cell types in B. tropicalis (Wu et al. 2009) too. Distinct spherite cells versus digestive cells as described by Sobotnik et al. (2008a) in A.siro were not seen. The disconcerting variety of acarine gut cells reported over many years by authors are only different functional agestages and degrees of maturity of the same cells (as in Liponyssus-Reichenow 1918). No evidence that the anterior parts of the gut acts in excretion (as in L.konoi Vijayambika and John 1975d) nor the hinder portion of the gut acting as an organ of nitrogenous excretion as in some arthropods (Lankester 1904) was found in P. longicornis.

The gut epidermal tissue is a stiff system ('Check (e)') — just like the pergamasid excretory system (Bowman 2017a). Gut architecture informs a narrative of gut process just as in ticks (Alberti and Coons 1999). Consilience of half-lives supports that cells appear to quickly stretch as a physical consequence of food ingestion by the predatory pergamasid to form a simple pavement epithelium. Ingestion half-lives are more than twice as fast as in egg-feeding amblyseiids (calculation from Table 7 in Blommers et al. 1977) and more than 60-80 times faster than tick ingestion (recalculations from Fig. 2 and Table III in Arthur 1965; and from Fig. 1 in Snow 1970). The plicate nature of the exterior of the starved gut unfolds as it is stretched (Neumann 1941) especially when ingesting a large amount of food (Vinogradova 1960; Lagutenko 1962; Akimov and Starovir 1978; Starovir 1980) resulting in the appearance of thin cells. Dilation (and physiological pace-see Table 9.1 in Sonenshine and Roe 1991) in pergamasids is more argasid-like than ixodid-like.

Digestion appears to be triggered by maximum gut expansion-estimated at 10 min post start of feeding ['Check (b)']. Early-on, gut cells typify fluid transport and possible enzyme secretion. Cells inflate or swell as a physical consequence of extension by fluid or food absorption. Gut cells shrink slowly as a physical consequence of degenerative egestionbut faster than excretory processes ['Check (e)']. Consilience of half lives supports that cell disappearance drives gut shrinkage. Akimov and Starovir (1977) poses that each cell only runs through one cycle culminating in its complete degeneration but that is contradicted by the ultrastructural results of El Shoura (1988). So, the detailed kinetics of egestion needs further biochemical investigation.

During pergamasid feeding, first fluid ingestion and then food absorption/digestion occurs. This does not exactly overlap with, but mirrors, the three stages Balashov (1961a) describes in argasids-i.e., an initial stage of no digestion while the gut epithelium is developing (here also fluid transport occurs and possible extracellular food lysis in the lumen happens); a second stage with the formation of an active epithelium when absorption and digestion takes place rapidly; finally a slow digestive (and eventually egestive and starvation) stage.

The state at time zero in this histological study of pergamasids was: no vacuolation and all three cell types present sporadically in the anterior mid-gut; but, all three cell types present with large vacuoles in the posterior mid-gut and hind-gut. In other words, one week's 
fasting at room temperature before investigation in pergamasids does not result in complete starvation. The true fasting/starvation state is of square and jigsaw-piece cells with no vacuoles (shown in the results $>2$ weeks after the start of feeding). Thin cells appear immediately on prey imbibition (as the gut expands-Bowman 2014) and become rapidly vacuolate in the later stages of imbibition around the end of the feeding period. Thereafter they disappear being switched into square cells.

Square cells without or with small or large vacuoles represent the main digestive 'engine' cell type giving way to vacuolate jigsaw-piece like (i.e., irregularly tessellated) cells as the gut contracts and the mid-gut lumen disappears. Devacuolation is faster than overall gut shrinkage. The gut zenith after a meal is many highly vacuolate jigsaw-piece like cells (resembling a facultative 'hepatopancreas' in the form of pseudo-stratified epithelium) which progressively devacuolate slowly-eventually through prolonged fasting/ starvation to the final loss of even the mid and hind-gut lumen. Tracking of lipids and free fatty acids quantitatively (as in ticks-Hajjar 1972; Maroun 1972, or hydrachnids Fried and Boddorff 1975) would be useful in confirming this in future work as arthropods appear to depend upon fatty acids rather than carbohydrates as their main source of energy (Maroun and Kamel 1973). Where mesostigmatids obtain their sterols from (Maroun and Kamel 1976) also needs elucidating.

Figures 17 and 22 summarises the gut cellular transitions, overall vacuolation and their relationship to lumenal contents (Bowman 2017b). Functional phases (see Fig. 13, upper) are: early fluid transportation $(0-1 \mathrm{~h})$ and extracellular activity (10-90 $\mathrm{min})$ [I and O]; through rising food absorption (10 min to $>1$ days) [D]; to slow meal intracellular processing, and degenerative egestive waste material production as a refractive 'dust' ( 1 to $>12$ days) [E].

Changes in cellular and lumenal predominances offer the opportunity for close coupling with Malpighian guanine production within a fixed idiosomal volume for this mite (see Fig. 18 Lower Row). The length of the digestive period in P. longicornis matches well the detection of prey in the gut of the related Typhlodromus pyri (of at least $31 \mathrm{~h}$ after start of feeding-Murray and Solomon 1978). From a contracted alumenate gut, jigsaw-piece like irregularly tessellated cells stretch during imbibition to form thin cells as the lumen appears. Prey foodstuff arrives in all parts of the gut simultaneously. These thin cells in turn swell over all gut regions synchronously as prey material is taken up intracellularly to form square cells (as the gut expands) and the latter become predominant after the end of feeding. The cells vacuolate over time during the digestive period. Square cells associated with granular (pale grey ellipse in Fig. 22, upper) and globular (grey ellipse with globules in Fig. 22, upper) lumenal prey contents, eventually shrink to form jigsaw (i.e., tessellated) cells during the egestive period with the appearance of refractive grains (dense grey granular ellipse in Fig. 22, upper) in the gut lumen. The midgut may be ready for a second meal within a few days before the first meal has been completely processed.

From around the time of the best estimate of initial gut emptying (12.5 h Bowman 2014) the gut as a whole has a disproportionately bigger lumen than its expansion/contraction status implies (see Fig. 20, lower) suggesting an initial drop in cellularity of its epithelium even as the gut begins to shrink - through to around 9 days after the start of feeding. This cell disappearance appears to be the origin of the egestive refractive grains (via apical or total) cellular degeneration. Detailed TEM observations are needed to confirm the production of residual bodies (or possibly spherites) and their release/disintegration to yield guanine, or uric acid or xanthine etc. Tracking this quantitatively as the gut shrinks and becomes alumenate would be useful. Such studies (Balashov and Raikhel 1973) have successfully elucidated the relationship of polysaccharide granules and guanine crystals in 

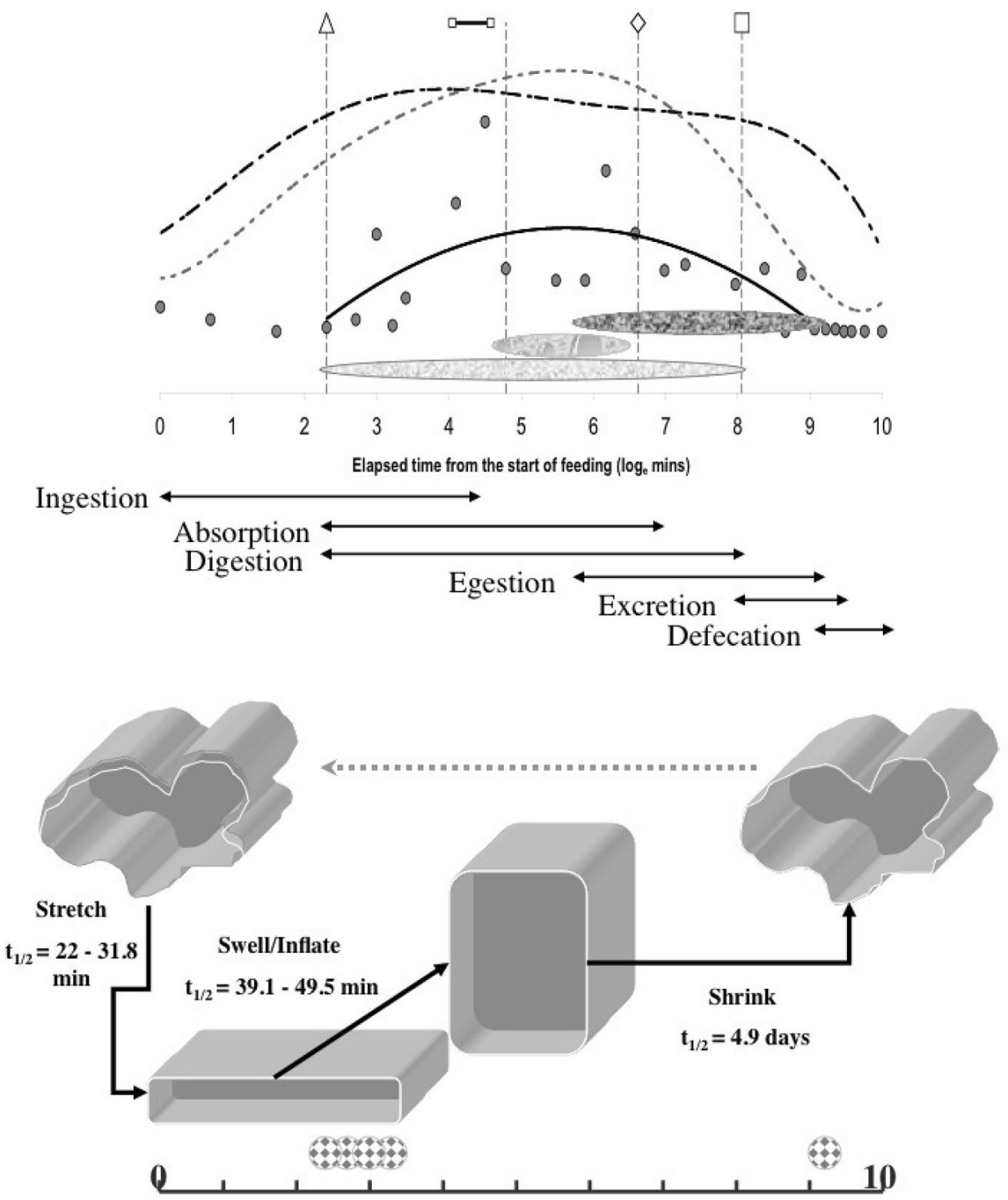

Fig. 22 Integrated cellular summary on $\ln (\mathrm{min})$ timescale after the start of feeding on larval dipteran prey in Pergamasus longicornis. Upper: time-course of lumenal contents (pale grey = fine granular imbibed prey material; medium grey = globular material; dark grey $=$ opaque micro-crystalline refractive grains (or 'dust'), from Bowman 2017b). Vacuolation over all cells (grey circles and quadratic trend over presence of vacuoles) showing rise and fall during digestion. Gut expansion/contraction mean score Bowman (2014) $=$ no symbol, upper heavy dashed grey (sixth order polynomial) trend line. Lumen presence/absence mean score $=$ no symbol and black dashed smoothed (fifth order polynomial) trend line Bowman (2017a). Symbols as in Fig. 7. Note vacuolation over period of granular and globular lumenal material. Lower: cellular schema with estimated half-lives (see Table 1) on same timescale. Solid lines indicate transitions (with halflives). Dotted grey line 'closes the loop' on the feeding to fasting cycle. 'Bead-let' free-cells (of unknown origin, not to scale) indicated at bottom. Jigsaw-like cells stretch to Thin cells which then inflate/grow to Square cells which finally shrink back to Jigsaw-piece like cells. New cells are produced from stem cells not scored in this study 
tick Malpighian tubules where the amount of guanine produced reflects metabolic activity (Hamdy 1973).

Jigsaw-piece like cells in a contracted alumenate mid/hind-gut represent the true fasted/ starved state-unlike in amblyseiids (Blommers et al. 1977). Zero gut content is reached as the ventriculus and caeca compress. Posteriorly these cells, in a pseudo-stratified transitional epithelium, may represent a long-term depot against hunger in the wild, functionally working in the same way as the decapod hepatopancreas (Gibson and Barker 1979) or the hepatopancreatic portion of the scorpion alimentary canal (Awati and Tembe 1952). As Malpighian tubules are present in anactinotrichid mites, the gut in P. longicornis does not have to act as a bona fide excretory organ as in some Trombidiformes (and oribatidsDinsdale 1975), nor as a temporary (Mitchell and Nadchatram 1969) or permanent waste dump as in mites with no anus. Whether in turn the Malpighian tubules in pergamasids have a food storage function (as fat depots-Lees 1964) remains to be confirmed. Excretion occurs in a definite cycle as in ticks (Enigk and Grittner 1952; Hamdy 1973).

As a heart is present in large gamasids (Kramer 1886; Winkler 1886) like in ticksRobinson and Davidson (1913-1914), it is not necessary for the mid-gut to perform a gastrovascular function as in trombidiform mites (Mitchell 1964) either. However, future work might cover the role of formed elements in the pergamasid haemocoel as in acarid mites (Kanungo and Naegele 1964) and argasid and ixodid ticks (Teravsky 1957; Tsvileneva 1959; Dolp 1970; Amosova 1983). There, the relative numbers of phagocytic trophocytes, macrophage-like phagocytes, prohaemocytes - progenitors of those two-and other intermediates like plasmatocytes and spherule cells also change with acarine's gut nutritional physiological state. How any haemocytes (Brinton and Burgdorfer 1971), amoebocytes (Berezantsev 1980), loose connective tissue (Tsvileneva 1961a, 1963), adipose tissue (Tsvileneva 1961b) or any sub-hypodermal guanocytes known in araneids (Seitz 1972) may synchronously change from feeding to fasting warrants investigation in pergamasids.

Like other arachnids, $P$. longicornis combines a mechanism of extra-corporeal prey liquefying with a subsequent completion of the digestion inside the midgut cells. Figure 13 confirms that the overall data maps well to a single time ordering of histological observations around a common process of: rapid rate-limiting ingestion; brisk concentrative osmoregulation ('coxal droplets' production); steady digestion; slow egestion; and, very slow excretion of prey material (see Fig. 21). This needs validating (and extending with NGS) in other easily available large non-phytoseiid predatory mesostigmatids - such as the polyphagous Parasitus fimetorum from intensive mushroom beds (Binns 1973), or Macrocheles muscadomesticae and Glyptholaspis confusa from cow manure (Axtell 1963) or Platyseius italicus from sewage filter beds (Barker 1961). Could more than just pergamasid physiological episodes be just like those expected from a 'non-blood feeding' argasid? Do solid feeding uropodines (Mesostigmata) show this anactinotrichid faciesor are they physiologically like cryptostigmatid actinotrichids? Whichever it is, using this whole scheme it would be possible for ecologists to probabilistically stage specimens of $P$. longicornis from the wild based upon their complete nutritive status (much like in ticksBalashov 1961b; Repkina 1976) given appropriate assays. Although the fine detail of the physiological and kinetic link between Malpighian excretion and gut food assimilation via modelling the haemocoel still awaits discernment, the close coupling of a primitive (acidic?) intracellular process with an advanced (alkaline?) extracellular process in a single digestive tissue marks the soil mite $P$. longicornis clearly out as a superbly adapted polyphagous predator. 
Acknowledgements The experimental setup and histological work in this study was funded by Science Research Council grant B/77307818 at Liverpool John Moores University. Thanks go to Susan Bjornson (then at University of Alberta, Canada) for advice on invertebrate sporozoans and Don E Johnston (Ohio State University) for confirming mite identifications. Data collection and analysis was self-funded. My late father Walter E Bowman prepared Fig. 13. I thank I A Akimov (Editor-in-Chief Vestnik Zoologii), Cambridge University Press, Elsevier, and Taylor \& Francis Ltd (http://www.tandfonline.com) for permission to reuse figures. I thank Tobias Töpfer and Håkon Haraldseide for permission to reuse their colour mite photographs. I thank two anonymous referees for generously highlighting various missed references. I thank Malcolm Luxton for his encouragement. Above all, I thank my wife Diane for supporting my involvement in this field over many years. The author is a Royal Society Industrial Fellow (IF110047) at Mathematical Institute University of Oxford. All data generated or analysed during this study at Oxford Centre for Industrial and Applied Mathematics and all model specifications are included in this published article.

\section{Compliance with ethical standards}

Conflict of interest The author declares that he has no known conflicts of interest. No competing claims are known.

Human or animal rights This article does not contain any studies with human participants or vertebrate animals performed by any of the authors.

Open Access This article is distributed under the terms of the Creative Commons Attribution 4.0 International License (http://creativecommons.org/licenses/by/4.0/), which permits unrestricted use, distribution, and reproduction in any medium, provided you give appropriate credit to the original author(s) and the source, provide a link to the Creative Commons license, and indicate if changes were made.

\section{References}

Aboul-Nasr AE, Bassal TTM (1971) Biochemical and physiological studies on certain ticks (Ixodoidea). The sugar content and concentration in Argas and Hyalomma biological fluids. J Med ENT 8(5):521-524

Adolph EF (1949) Quantitative relations in the physical constitutions of mammals. Science 109:579-585

Aeschlimann A, Ryhiner RM (1970) Note sur une particularité anatomique du systéme digestif chez Ornithodorus moubata Murray (Ixodoidea:Argasidae). Acat Trop 27(2):191-192

Aeschlimann A, Grandjean G (1973) Influence of natural and "artificial" mating on feeding, digestion, vitellogenesis and oviposition in ticks (Ixodoidea). Folia Parasitol (Prague) 20(1):67-74

Agbede RI (1986) Scanning electron microscopy of digest cells in the mid-gut epithelium of Boophilus microplus. Exp Appl Acarol 2(4):329-335

Agbede RI, Kemp DH (1985) Digestion in the cattle-tick Boophilus microplus: light microscope study of the gut cells in nymphs and females. Int J Parasitol 15(2):147-157

Agbede RI, Kemp DH (1987) Ultrastructure of secretory cells in the gut of the cattle-tick Boophilus microplus. Int J Parasitol 17(6):1089-1098

Agyei AD, Runham NW (1995) Studies on the morphological changes in the mid-guts of two ixodid tick species Boophilus microplus and Rhipicephalus appendiculatus during digestion of the blood meal. Int J Parasitol 25(1):55-62

Agyei AD, Herbert IV, Runham NW (1991) Histochemical localisation of acid phosphatase and non-specific esterase in the mid-guts of two species of tick, Boophilus microplus and Rhipicephalus appendiculatus, as determined by light microscopy. Parasitol Res 77(7):629-634

Agyei AD, Runham NW, Blackstock N (1992) Histochemical changes in the mid-gut of two ixodid tick species Boophilus microplus and Rhipicephalus appendiculatus during digestion of the blood meal. Exp Appl Acarol 13(3):187-212

Ahearn GA, Hadley NF (1977) Water transport in perfused scorpion ileum. Am J Physiol 233(5):R198-R207

Ainscough BD (1960) The internal morphology of Caminella peraphora Krantz and Ainscough, with descriptions of the immature stages (Acarina: Trachytidae). Unpublished Master of Science Thesis, Oregon State University College LD4330 
Akimov IA (1973) On the morphological and physiological characteristics of the alimentary canal of the bulb mite Rhizoglyphus echinopus. In: Proceedings of the 3rd international congress of acarology prague, Aug 31-Sept 6. 1971. The Hague, The Netherlands, pp 703-706

Akimov IA (1975) Morphology of the digestive system of Rhizoglyphus echinopus (Fumouze et Robin) (Acariformes, Acaroidea). Vestnik Zoologii 0(3):66-72 (in Russian)

Akimov IA (1977) Extracellular, contact and intra-cellular digestion in acarid ticks. Vestnik Zoologii 0(4):89-90 (in Russian)

Akimov IA (1980) Functions of the gut sections in acaroid mites (Acaroidea). Vestnik Zoologii 0(3):17-24 (in Russian)

Akimov IA, Barabanova VV (1976a) The digestive enzymes of certain acaroid mites. Dopovidi Akad Nauk Ukr RSR Seriya B Heol Khim Biol Nauky 0(6):547-550 (in Ukrainian)

Akimov IA, Barabanova VV (1976b) Some aspects of digestion in the mites Cosmoglyphus absoloni Samsinak, 1961 and Coleochaeta molitor Volgin et Akimov, 1975. Dopovidi Akad Nauk Ukr RSR Seriya B Heol Khim Biol Nauky 0(7):643-645 (in Ukrainian)

Akimov IA, Barabanova VV (1977) Morphological and functional characteristics of the digestive system in tetranychid mites (Trombidiformes, Tetranychoidea). Entomol Rev 56 (4):141-148 (translation of Entomologischeskoe Obozrenie 56(4):912-922 in Russian)

Akimov IA, Barabanova VV (1978) The influence of the feeding to the activity of the digestive enzymes of acaroid mites. Ekologiya 0(2):27-31 ((in Russian) translated cover to cover as Soviet Journal of Ecology 0(2):117-120)

Akimov IA, Barabanova VV (1990) Seasonal digestion activity changes in Varroa jacobsoni females. Vestnik Zoologii 0(1):39-42 (in Russian)

Akimov IA, Dei EA (1973) The appearance of degenerated digestive cells with undigested food residues in the body cavity of spider mites Tetranychus cinnabarinus Boisduval (1867). Vestnik Zoologii 7(3):78 (in Russian)

Akimov IA, Gorgol' VT (1984) Structure and functions of the digestive system of Bakericheyla chenayi (Cheyletida, Trombidiformes), an ectoparasite of birds. Parazitologiya 18(1):15-22

Akimov IA, Gorgol' VT (1987) Morphological and functional grounds of trophic specialization of a predaceous mite, Cheyletus eruditius (Trombidiformes, Cheyletidae). Vestnik Zoologii 0(5):49-54 (in Russian)

Akimov IA, Schlur LE (1972) Characteristics of the nutrition of the mites Glycyphagus domesticus, Tyrophagus putrescentiae and Rhizoglyphus echinopus, with some proteinoids. Vestnik Zoologii 0(6):45-48 (in Russian)

Akimov IA, Starovir IS (1974) Morpho-functional features of the digestive system in Phytoseiulus persimilis Athias-Henriot (Gamasoidea. Phytoseiidae). Vestnik Zoologii 0(4):60-64 (in Russian)

Akimov IA, Starovir IS (1976) Structure of the digestive system of the mites Amblyseius andersoni and Amblyseius reductus (Parasitiformes. Phytoseiidae). Vestnik Zoologii 0(4):7-13 (in Russian)

Akimov IA, Starovir IS (1977) Morpho-functional features of the digestive system of the mite Amblyseius andersoni (Gamasoidea. Phytoseiidae). Vestnik Zoologii 0(3):82-86 (in Russian)

Akimov IA, Starovir IS (1978) Morpho-functional adaptation of digestive system of three species of Phytoseiidae (Parasitiformes, Phytoseiidae) to predatoriness. Dopovidi Akad Nauk Ukrainian RSR Seriya B Heol Khim Biol Nauky 7:635-641 (in Ukrainian)

Akimov IA, Starovir IS (1980) The structure of the digestive system in Acotyledon absoloni Samsinak, 1961 (Acariformes, Acaroidea) dwelling in termitaria. Vestnik Zoologii 0(1):51-56 (in Russian)

Akimov IA, Starovir IS (1983) Digestive system structure in Varroa jakobsoni, a honey-bee parasite. Vestnik Zoologii 0(3):51-57 (in Russian)

Akimov IA, Yastrebtsov AV (1984) Reproductive system of Varroa jacobsoni. I. Female reproductive system and oogenesis. Vestnik Zoologii 0(6):61-68 (in Russian)

Akimov IA, Yastrebtsov AV (1985) Reproductive system of Varroa jacobsoni. II. Male reproductive system. Vestnik Zoologii 0(2):63-69 (in Russian)

Akimov IA, Oksentyuk YaR (2018) Functional and ecological adaptations of several acaridid mite species (Acariformes, Astigmata) for feeding on stored produce. Vestnik Zoologii 52(4):323-330. https://doi. org/10.2478/vzoo-2018-0034

Akimov IA, Piletskaya IV, Yastrebtsov AV (1988) Morpho-functional age changes in the reproductive system of female Varroa jacobsoni. Vestnik Zoologii 0(6):48-55 (in Russian)

Akov S, Samish M, Galun R (1976) Protease activity in female Ornithodoros tholazani ticks. Acta Trop 33(1):37-52

Alberti G (1972) Vergleichende bionomische und anatomische Untersuchungen an Schnabelmilben (Fam. Bdellidae). Dissertation zur Erlangung des Doktorgrades der Mathematisch-Naturwissenschaftlichen Fakultät der Christian-Albrechts-Universität zu Kiel 141pp 
Alberti G (1973) Ernährungsbiologie und Spinnvermögen der Schnabelmilben (Bdellidae, Trombidiformes). Z Morphol Tiere 76:285-338

Alberti G (1977) Zur Feinstruktur und Funktion der Genitalnäpfe von Hydroma despiciens (Hydrachnellae, Acari). Zoomorphologie 87:155-164

Alberti G (1979) Licht und elektronenmikroskopische Untersuchungen an Coxaldrúsen von Walzenspinnen (Arachnida: Solifugae). Zool Anz 203(1-2):48-64

Alberti G (1980) Zur Feinstruktur der Spermien und Spermiocytogenese der Milben (Acari). I Anactinotrichida. Zool Jb Anat 104:77-138

Alberti G (1980) Zur Feinstruktur der Spermien und Spermiocytogenese der Milben (Acari). II Actinotrichida. Zool Jb Anat 104:144-203

Alberti G, Coons LB (1999) Acari: mites. In: Harrison FW, Foelix RF (eds) Microscopic anatomy of invertebrates, vol 8C. Wiley, New York, pp 515-1215

Alberti G, Hänel H (1986) Fine structure of the genital system in the bee parasite, Varroa jacobsoni (Gamasida: Dermanyssina) with remarks on spermiogenesis, spermatozoa and capacitation. Exp Appl Acarol 2(1):63-104

Alberti G, Storch V (1973) Zur Feinstruktur der "Munddrüsen" von Schnabelmilben (Bdellidae, trombidiformes). Zwiss Zool 186(1-2):149-160

Alberti G, Storch V (1983) Zur Ultrastruktur der Mitteldarmdrüsen von Spinnentieren (Scorpiones, Araneae, Acari ) unter verschiedenen Ernährungsbedinungen. Zool Anzeiger 11:145-160

Alberti G, Kaiser T, Klauer A-K (1996) New ultra structural observations of the coxal glands (Nephridia) of Acari. In: Mitchell RD (ed) Acarology XI. Proceedings of IX international congress of acarology, held July 17-22, 1994, at Ohio State University. Ohio Biological Survey, Columbus, Ohio, pp 309-318

Alberti G, Gegner A, Witalinski W (1999) Observations on the fine structure of the vagina of pergamasid mites (Pergamasidae, Parasitina, Gamasida). In: Bruin J, van der Geest LPS, Sabelis MW (eds) Ecology and evolution of the acari. In: Proceedings of the 3rd symposium of the European association of acarologists 1-5 July 1996. Springer, Amsterdam, pp 593-601

Alberti G, Seniczak A, Seniczak S (2003) The digestive system and fat body of an early-derivative oribatid mite, Archegozetes longisetosus Aoki (Acari: Oribatida, Trhypochthoniidae). Acarologia 43(1):149-219

Alberti G, de Andrade D, Garita LC, Kitajima EW (2014) Anatomy and fine structure of Brevipalpus mites(Tenuipalpidae)—economically important plant-virus vectors-part 3: digestive system. Zoologica 160:67-94

Allen WE (1905) Internal morphology of the American Cattle Tick. Trans Am Microsc Soc 26:245-280 (studies from Zoological Lab Univ Nebraska, No 67)

Amosova LI (1983) Ultrastructural characters of hemocytes of the ixodid tick, Hyalomma asiaticum (Ixodidae). Parazitologiya 17(2):126-133

Anderson JF (1966) The excreta of spiders. Comp Biochem Physiol 17(3):973-982. https://doi. org/10.1016/0010-406X(66)90136-8

André M (1927) Digestion "extra-intestinale" chez le Rouget (Leptus autumnalis Shaw). Bull Mus Nationale Hist Naturelle Paris 33:509-516

André M (1949) Ordres des Acariens. (Acari, Nitzsch, 1818). In: Grassé P-P Traité de Zoologie. Onychophores, Tardigrades, Arthropodes, Trilobitomorphes, Chélicérates. Éditions Masson 6:794-892

Andrew W (1959) Textbook of comparative histology. Oxford University Press, Oxford

Anwarullah W (1963) Beiträge zur Morphologie und Anatomie einiger Tetranychiden (Acari, Tetranychidae). Zeitschrift fur angewandte Zoologie 50(4):385-426

Arthur DR (1965) Feeding in ectoparasitic Acari with special reference to ticks. Adv Parasitol 3:249-298

Ashton MK (1961) A study of the functional anatomy of the brown wheat mite, Petrobia latens (Muller). J Colo Wyo Acad Sci 5:50-51

Awati PR, Tembe VB (1952) Buthus tamulus (Fabr) (The Indian Scorpion) (morphology, anatomy \& bionomics). Bombay University-zoological memoirs no. 2

Axtell RC (1963) Acarina occurring in domestic animal manure. Ann Entomol Soc Am 56(5):628-633

Bader C (1938) Beiträge zur Kenntnis der Verdauungsvorgänge bei Hydrachniden. Revue Suisse Zoologie 45(22):721-806

Bader C (1954) Das Darmsystem der Hydracarinen. Eine vergleichend-anatomische Untersuchungen. Revue Suisse Zool 61(29):505-549

Bader C (1969) Contributions to the taxonomy of water mites. In: Evans GO (ed) Proceedings of the 2nd international congress of acarology. Akademiai Kiado, Budapest, pp 89-92

Barker RA (1961) A preliminary survey of the mite fauna of sewage percolating filters. Thesis, University of London (Birkbeck College), M.Sc 
Baker RA (1970) The food of Riccardoella limacum (Schrank)_Acari-Trombidiformes and its relationship with pulmonate molluscs. J Nat Hist 4:521-530

Baker RA (1971) Observations on aspects of nutrition in Xenopacarus africanus Fain, Baker \& Tinsley (Ereynetidae: Trombidiformes). J Med Entomol 8(3):307-313

Baker RA (1973) Notes on the internal anatomy, the food requirements and development in the family Ereynetidae (Trombidiformes). Acarologia 15(1):43-52

Baker RA (1975) The structure and function of the alimentary canal in Histiogaster carpio (Kramer, 1881) Acari-Sarcoptiformes. Acarologia 17(1):126-137

Baker RA (1977) Nutrition of the mite Unionicola intermedia, Koenike and its relationship to the inflammatory response induced in its molluscan host Anodonta anatina L. Parasitology 75:301-308

Baker RA, Whittington B (1980) Microorganisms associated with the gut of Dermanyssus gallinae the Poultry mite. Parasitology 81(2):XXV

Balashov YuS (1957a) Histological features of digestion in the Ixodidae and Argasidae ticks. Parazitologicheskii Sbornik Zoologischeskogo Instituta AN SSSR 17:137-167 (in Russian)

Balashov YuS (1957b) Certain adjustments to the reception of large blood masses in the ixodid ticks. Zool Zh 36:870-873 (in Russian)

Balashov YuS (1961a) The structure of digestive organs and the blood digestion in argasidae. Mag Parasit Moscow 20:185-225 (in Russian)

Balashov YuS (1961b) Dynamics of stored nutritive substances and age determination in hungry ixodid ticks. Zool Zh 40:1354-1363

Balashov Yu S (1962) Gonotrophic relations in argasid ticks. Voprosy Obshch Zool i Med Parazitol, Moskva, pp 380-401 (in Russian)

Balashov YuS (1964) Destruction of vertebrate erythrocytes in the alimentary canal of blood-sucking arthropods. Proc Acad Sci USSR Biol Sci Sect 151:1024-1028 (in Russian)

Balashov YuS (1972) Bloodsucking ticks (Ixodoidea). Vectors of diseases of man and animals. Misc Publ Ent Soc Am 8:161-376

Balashov YuS (1988) Ultrastructural study of the internal organs of ixodid ticks in the scanning electron microscope. Parazitologiya 22(6):513-515 (in Russian)

Balashov YuS, Raikhel AS (1973) Electron microscope studies of Malpighian tubules in Hyalomma asiaticum. Part 1 hungry females. Parazitologiya, Leningr 7(3):231-237 (in Russian)

Balashov YuS, Raikhel AS (1974) Ultrastructure of the mid-gut in hungry nymphs of the tick Hyalomma asiaticum acarina ixodidae. Zool Zh 53(8):1161-1168 (in Russian)

Balashov YuS, Raikhel AS (1975) Electron microscopic study of the excretory system of hungry females of the tick Hyalomma asiaticum. Part 2. Parazitologiya (Leningr) 9(3):252-259 (in Russian)

Balashov YuS, Raikhel AS (1976a) Ultrastructure of the mid-gut epithelium in nymphs of the tick Hyalomma asiaticum during feeding. Parazitologiya Leningr 10(3):201-209 (in Russian)

Balashov YuS, Raikhel AS (1976b) Ultrastructure of the excretory system in the argasid tick Ornithodoros papillipes. Parazitologiya 10(5):385-391 (in Russian)

Balashov YuS, Raikhel AS (1977) Ultrastructure of the mid-gut of hungry nymphs of Ornithodoros papillipes (Acarina, Argasidae) Parazitologiya 11(2):122-128 (in Russian)

Balashov YuS, Raikhel AS (1978) Ultrastructure of the mid-gut in nymphs of Ornithodoros papillipes (Acarina, Argasidae) during blood assimilation. Parazitologiya 12(1):21-26 (in Russian)

Barabanova VV (1972) Some digestive enzymes of Tetranychus cinnabarinus Boisduval (Tetranychidae). Vestnik Zoologii 0(6):89-90 (in Russian)

Barabanova VV (1973) Digestion of starch and protein by Rhizoglyphus echinopus. Vestnik Zoologii 0(6):81-82 (in Russian)

Barabanova VV (1975) Some digestive enzymes of mites of the genus Tetranychus. Dopovidi Akad Nauk Ukr RSR Seriya B Heol Khim Biol Nauky 0(11):1028-1030 (in Ukrainian)

Barabanova VV (1976) Adaptation of the mite Rhizoglyphus echniopus to a new food substrate. Vestnik Zoologii 0(6):80-82 (in Russian)

Barabanova VV (1980a) On possibility to digest some food substrates by Androlaelaps casalis. Vestnik Zoologii 0(3):93-95 (in Russian)

Barabanova VV (1980b) Digestion peculiarities in certain mites of the Phytoseiidae family (Gamasina, Phytoseiidae). Vestnik Zoologii 0(5):92-96 (in Russian)

Barabanova VV (1981) Some digestive enzymes of the mite Chaetodactylus osmiae Acaroidea Glycyphagidae. Vestnik Zoologii 0(2):88-89 (in Russian)

Barabanova VV (1983) Digestive enzymes of Varroa jakobsoni. Vestnik Zoologii 0(3):81-83 (in Russian)

Barabanova VV (1984a) Intestinal proteolytic activity in Varroa jacobsoni females. Vestnik Zoologii 0(1):69-72 
Barabanova VV (1984b) Variation of digestive enzymes activity in varroa mites during ontogenesis. Vestnik Zoologii 0(5):76-79 (in Russian)

Barabanova VV (1985) Specificity of some intestinal enzymatic systems in predaceous mites Phytoseiulus persimilis and Amblyseius longispinosus. Vestnik Zoologii 0(5):52-57 (in Russian)

Barabanova VV (1991) Ecological and physiological peculiarities of the Varroa jacobsoni female mites starvation. Vestnik Zoologii 0(6):54-57 (in Russian)

Barabanova VV (1993) Some digestive peculiarities of the mite Anystis baccarum. Vestnik Zoologii 0(2):68-71 (in Russian)

Bardi JK, George CJ (1943) Digestive glands of the scorpion. A physiological investigation. J Univ Bombay 11:91-114

Barker RJ, Lehner Y (1976) Sugars in hemolymph of ticks. J Med ENT 13(3):379-380

Barry DH, Mawdesley-Thomas LE (1968) Enzyme histochemistry of the adult liver fluke, Fasciola hepatica. Exp Parasitol 23:355-360

Behura BK (1956) The feeding mechanism of Histostoma polypori. Proc Zool Soc 9(1):1-11

Bekker EG (1940) The structure, role and origin of connective tissue in the body cavity of grain mites (Tyroglyphidae). Uchen ap mosk Gosud Univ Zool (Sci Rep Moscow State Univ Zool) 42:99-112 (in Russian)

Bekker EG (1959) To the problem of the order Acarina. Trudy Inst Morfol Zhivotnykh im AN Severtsova Akad Nauk SSSR 27:151-162 (in Russian)

Belozerov VN (1957) Biology and anatomy of the mite Poecilochirus necrophori Vitz. (Parasitiformes, Parasitidae). Zool Zh 36(12):1802-1813 (in Russian)

Belozerov VN, Tymopheev VA (1973) An electron microscopic investigation of the mid gut epithelium of the adult female of Dermacentor marginatus. In: Daniel M, Rosicky B (eds) Proceedings of the 3rd international congress of acarology, Prague, Czechoslovakia Aug 31-Sept 6, 1971, pp 703-709

Bensoussan N, Santamaria ME, Zhurov V, Diaz I, Grbić M, Grbić V (2016) Plant-herbivore interaction: dissection of the cellular pattern of Tetranychus urticae feeding on the host plant. Front Plant Sci 7(1105):1-13. https://doi.org/10.3389/fpls.2016.01105

Bensoussan N, Zhurov V, Yamakawa S, O’Neil CH, Suzuki T, Grbić M, Grbić V (2018) The digestive system of the two-spotted spider mite, Tetranychus urticae (Koch), in the context of the mite-plant interaction. Front Plant Sci 9(1206):1-18. https://doi.org/10.3389/fpls.2018.01206

Berezantsev AYu (1980) Anatomy of adult phases of the mites, Campyloythrombium boreale and Echinothrombium spinosus (Acarina, Trombiculidae). Vestn Leningr Univ Biol 0(1):14-21

Berlese A (1897) Ricerche sugli organi e sulla funzione della digestione negli Acari. Rivista di Patologia vegetale 5:129-195

Berlese A (1899) Circa il mesointestino di alcuni aracnidi. Rivista di patologica vegetale 7:226-248

Berlese A (1906) Monografia del Genere Gamasus Latr. Redia 3(1):65-304

Berlese A (1918) Intorno agli Uropodidae. Redia 13:7-16

Bernard HM (1893) VIII. Notes on some of the digestive processes in arachnids. J R Microsc Soc 13:427-443

Bernini F (1971) Ultrastructural observations on the alimentary canal in Oribatidae (Acari). In: Daniel M, Rosicky B (eds) Proceedings of the 3rd international congress of acarology, Prague, Czechoslovakia Aug 31-Sept 6, 1971, pp 67-71

Bertkau Ph (1884) Über den Bau und die Funktion der sog. Leber bei den Spinnen. Arch für mikrosk Anat 23:214-245

Bertkau Ph (1885) Über den Verdauungsapparat der Spinnen. Arch für mikrosk Anat 24:398-451

Bhattacharyya SK (1962) Laboratory studies on the feeding habits and life cycles of soil-inhabiting mites. Pedobiologia 1:291-298

Bhattacharyya SK (1963) A revision of the British mites of the genus Pergamasus Berlese s.lat. (Acari: Mesostigmata). Bull Brit Mus (Nat Hist) Zool 11(2):133-242

Biedermann W (1911) Die Aufnahme, Verarbeitung und Assimilation der Nahrung. H Winterstein's Handbuch der vergleichenden Physiologie Gustav Fischer Jena 2(1):1-1563

Binns ES (1973) Predatory mites—neglected allies? Mushroom J 12:3-7

Birula A (1891) Einiges über den Mitteldarm der Galeodiden. Biol Zentrallbl 11(9-10):295-300

Blackith RM (1975) Interrelationships between small arthropods and nematodes in peat. Proc R Ir Acad B 75(26):531-541

Blanc G (1910) Sur la termination du tube digestive des Ixodidae. Bull Soc Zool Fr 35:219-225

Blanchard E (1855a) L'Organisation du Règne animal Arachnides. C R Acad Sci Paris 41:1256

Blanchard E (1855b) Des fonctions du foie chez les Arachnides. C R Acad Sci Paris 41:1256-1258

Blauvelt WE (1945) The internal morphology of the common red spider mite (Tetranychus telarius Linn.). Mem Cornell Agric Exp St No 270:1-35 
Blommers L, Lobbes P, Vink P, Wegdam F (1977) Studies on the response of Amblyseius bibens [Acarina: Phytoseiidae] to conditions of prey scarcity. Entomophaga 22(3):247-258

Boctor FN, Araman SF (1972) Biochemical and physiological studies of certain ticks (Ixodoidea). Total free amino-acids in gut hemolymph and coxal fluids of Argas (Persicargas) persicus (Oken) and Argas (Persicargas) arboreus K.H.K. Argasidae. J Med Entomol 8(5):525-528

Boczek J, Jura C, Krzysztofowicz A (1969) The comparison of the structure of the internal organs of post embryonic stages of Acarus farris with special reference to the hypopus. In: Proceedings of the 2 nd international congress of acarology, pp 265-271

Bogin E, Hadani A (1973) Digestive enzymes in "Hard Ticks" (Ixodoidea, Ixodidae). I. Proteolytic enzyme activity in the gut of Hyalomma excavatum female ticks. Z Parasitenkd 41(2):139-146

Bonnet A (1906) Sur l'anatomie et l'histologie des Ixodes. Compte Rendus Hebd des Séances de l'Acad Sci Paris 142:296-298

Bonnet A (1907) Recherches sur l'antomie comparée et le développement des Ixodidés. Annales de l'Universiteé de Lyon, Nouvelle série, 1. Sciences, Meédecine Fasc 20:1-174

Bonnet A (1908) L'anatomie et la classification des Ixodes. Archives de Parasitologie 12(2):224-267

Bowman CE (1981) Hide protease in stored product mites (Astigmata: Acaridae). Comp Biochem Physiol B Comp Biochem 70(4):803-805

Bowman CE (1984a) Some aspects of feeding and digestion in the soil predatory mite Pergamasus longicornis (Berlese) (Parasitidae: Mesostigmata). In: Griffiths DA, Bowman CE (eds) Acarology VI. Ellis Horwood, Chichester, vol 1, pp 316-322

Bowman CE (1984b) Comparative enzymology of economically important astigmatid mites In: Griffiths DA, Bowman CE (eds) 1984 Acarology VI, vol 2. Wiley, Chichester, pp 993-1001

Bowman CE (1985) Studies of the protein content of individual Pergamasus longicornis (Berlese) (Acari: Mesostigmata: Parasitidae). Exp Appl Acarol 1(4):345-355

Bowman CE (1987a) Studies on feeding in the soil predatory mite Pergamasus longicornis (Berlese) (Mesostigmata: Parasitidae) using dipteran and microarthropod prey. Exp Appl Acarol 3:201-206

Bowman CE (1987b) Digestion in the soil predatory mite Pergamasus longicornis (Berlese) (Acari: Mesostigmata: Parasitidae)—detectable hydrolases. Comp Biochem Physiol B Comp Biochem 86(2):385-391

Bowman CE (2014) Gut expansion and contraction in the predatory soil mite Pergamasus longicornis (Mesostigmata: Parasitidae): a stiff system. Exp Appl Acarol 64(3):337-359

Bowman CE (2015) Individualised divergences. In: Dryden IL, Kent JT (eds) Geometry driven statistics. Wiley, New York, pp 337-355

Bowman CE (2017a) Modelling Malpighian tubule crystals within the predatory soil mite Pergamasus longicornis (Mesostigmata: Parasitidae). Exp Appl Acarol 72(1):35-59. https://doi.org/10.1007/s1049 3-017-0137-7

Bowman CE (2017b) Gut contents, digestive half-lives and feeding state prediction in the soil predatory mite Pergamasus longicornis (Mesostigmata: Parasitidae). Exp Appl Acarol 73 1):11-60. https://doi. org/10.1007/s10493-017-0174-2

Boyer PD (1971) The enzymes, vol IV. Hydrolysis, 3rd edn. Academic Press, New York

Bradford-Hill A (1965) The environment and disease: association or causation? Proc R Soc Med 58:295-300

Bregetova NG (1979) Some archaic and specialized features in structure and biology of Mesostigmatid mites (Acarina: Parasitiformes, Mesostigmata). In: Proceedings of the 4th international congress acarology, 1974, pp 447-451

Brinton LP, Burgdorfer W (1971) Fine structure of normal hemocytes in Dermacentor andersoni Stiles (Acari: Ixodidae). J Parasitol 57:1110-1127

Brody AR (1971) Microscopic anatomy of the house dust mite. Proc Natl Cent Br ENT Soc Am 26:64-65

Brody AR, Wharton GW (1970) Dermatophagoides farinae: ultrastructure of lateral opisthosomal dermal glands. Trans Am Microsc Soc 89(4):499-513

Brody AR, McGrath JC, Wharton GW (1972) Dermatophagoides farinae: the digestive system. J N Y Entomol Soc 80(3):152-177

Brody AR, McGrath JC, Wharton GW (1976) Dermatophagoides farinae: the supracoxal glands. J N Y Entomol Soc 84(1):34-47

Brown JRC (1952) The feeding organs of the adult of the common "chigger". J Morphol 91(1):15-51. https ://doi.org/10.1002/jmor.1050910103

Brown M (1922) Notes on the structure of an endoparasitic water-mite larva occurring in frogs. Washington University Studies. Sci Ser 9(2):291-315

Brucker EA (1900) Monographie de Pediculoides ventricosus Newport et theorie des pièces buccales des Acariens. Bull Sci Fr Belg 35:355-442 
Bücking J (2002) Three-dimensional structure of the gut system of the mites Ameronothrus lineatus (Oribatida: Ameronothridae) and Hyadesia fusca (Astigmata: Hyadesiidae). In: Bernini F, Nanelli R, Nuzzaci E de Lillo (eds) Acarid phylogeny and evolution. Adaptations in mites and ticks. Kluwer Academic Publishers, Norwell, pp 217-225

Buddenbrock W von (1956) Vergleichende Physiologie. Band III. Ernährung, Wasserhaushalt und Minerlahaushalt der Tiere. Birkh"auser Verlag, Basel und Stuttgart

Burgdorfer W, Brinton LP, Hughes LE (1973) Isolation and characterization of symbiotes from the Rocky Mountain Wood Tick, Dermacentor andersoni. J Invertebr Pathol 22(3):424-434

Burstone MS, Folk JE (1956) Histochemical demonstration of aminopeptidase. J Histochem Cytochem 4:217-226

Butler JF (1964) The biology and morphology of the mite Macrocheles muscadomesticae (Scopoli, 1772). MS Thesis, University of Wyoming, Laramie

Butler J, Tonn RJ (1963) Free amino acids in three species of mites. Ann ENT Soc Am 56(3):406

Caperucci D, Camargo Mathias M, Bechara GH (2009) Histopathology and ultrastructure features of the mid-gut of adult females of the tick Amblyomma cajennense Fabricius, 1787 (Acari: Ixodidae) in various feeding stages and submitted to three infestations. Ultrastuct Pathol 33(6):249-259

Caperucci D, Bechara GH, Camargo Mathias M (2010) Ultrastructure features of the mid-gut of the female adult Amblyomma cajennense ticks Fabricius, 1787 (Acari: Ixodidae) in several feeding stages and subjected to three infestations. Micron 41(7):710-721

Carter A, Cragg JB (1977) Potassium and calcium cycling by Eupterotegmaeus rostratus (Acari Cryptostigmata). Pedobiologia 17(3):169-174

Chaika SYu (1977) The ultrastructure of the glycocalyx of micro villi in the mid gut of blood sucking arthropods. Tsitologiya 19(11):1221-1224 (in Russian)

Chant DA (1958) On the ecology of typhlodromids in southeastern England. In: Proceedings of the 10th international congress ENT, vol 4, pp 649-658

Cherry LM (1976) Utilization of cholesterol by the cattle tick Boophilus microplus: cholesterol economy in the engorged female adult (Acari, Ixodidae). Insect Biochem 6(6):587-594

Chinery WA (1964) The midgut epithelium of the tick Haemaphysalis spinigera Neumann 1897. J Med ENT 1(2):206-212

Christophers SR (1906) The anatomy and histology of ticks. Sci mem by Officers of the Med Sanit Depts, Govt India, Calcutta (n.s.) 23:1-56

Čiampor F, Nosek J (1976) Virus and rickettsia like structures in gut cells of Dermacentor marginatus nymphs. Acta Virol Praha 20(5):439-441

Claparède E (1868) Studien an Acariden. Z wiss Zool 18:445-546

Collatz K-G, Mommsen T (1974) Die Struktur der emulgierenden Substanzen verschiedener Invertebraten. J Comp Physiol B Metab Transp Funct 94(4):339-352

Congdon P (2001) Bayesian statistical modelling. Wiley, Chichester

Coons LB (1978) Fine structure of the digestive system of Macrocheles muscaedomesticae (Scopoli) (Acarina: Mesostigmata). Int J Insect Morphol Embryol 7(2):137-154

Coons LB, Alberti G (1999) Acari-Ticks. In: Harrison FW, Foelix RF (eds) Microscopic anatomy of invertebrates. Chelicerate Arthropoda, vols 8B. Wiley, New York, pp 267-514

Coons LB, Axtell RC (1971a) Ultrastructure of the excretory tubes of the mite Macrocheles muscadomesticae with notes on altered mitochondria. J Morphol 133(3):319-337

Coons LB, Axtell RC (1971b) Cellular organization in the synganglion of the mite Macrocheles muscadomesticae. Ultrastructure of the excretory tubes of the mite Macrocheles muscadomesticae. Zeitschrift Zellforsch 119:309-320

Coons LB, Roshdy MA (1973) Fine structure of the salivary glands of unfed Dermacentor variabilis. J Parasitol 59:900-912

Coons LB, Rosell-Davis R, Tarnowski BI (1986) Blood meal digestion in ticks. In: Sauer JR, Hair JA (eds) Morphology, physiology and behavioral biology of ticks. Ellis Horwood, Chichester, pp 248-279

Coons LB, L'Amoreuax WJ, Rosell-Davis R, Starr-Spires L (1990) Fine structure of the fat body and nephrocytes in the life stages of Dermacentor variabilis. Exp Appl Acarol 8(1-2):125-142

Coruzzi L, Witkus R, Vernon GM (1982) Function related structural characters and their modifications in the hind gut epithelium of 2 terrestrial isopods Armadillidium vulgare and Oniscus assellus. Exp Cell Biol 50(4):229-240

Couch TL, Benton AW (1971) Comparison of gut and hemolymph enzymes and proteins of several species of spiders. Ann Ent Soc Am 64:409-413

Cromack K Jr., Solins P, Todd RL, Fogel R, Todd AW, Fender WM, Crossley ME, Crossley DA Jr (1977) The role of oxalic acid and bicarbonate in calcium cycling by fungi and bacteria: some possible implications for soil animals. In: Lohm U, Persson T (eds) Soil organisms as components of ecosystems. 
International soil zoology colloquium, Uppsala, Sweden June 21-25, 1976. Ecological BulletinSwedish Natural Science Research Council (Stockholm) 25:246-252

Croneberg A (1878a) Über den Bau von Eylais extendens (O.F.Müll.) nebst Bemerkungen über verwandte Formen. Nachrichten d Ges d Freunde für Naturkunde, Moskau 29 (in Russian)

Croneberg A (1878b) Über den Bau der Hydrachniden. Zool Anz 14:316-319

Croneberg A (1879) Über den Bau von Trombidium. Bulletin de la Soc Imp d Nat de Moscou 1:234-252

Crooker AR (1980) Fine structure of the adult female two-spotted spider mite Tetranychus urticae Acarina Tetranychidae. Am Zool 20(4):894

Daiber M (1921) II. Unterstamm oder Subphylum: Arthropoda pulmonata. III Hauptklasse: Arachnoidea sive Chelicerota. Spinnenartige Gliederfüßler. In: Lang A, Handbuch der Morphologie der wirbellosen Tiere. Vierter Band. Arthropoda, pp 269-350

Davidson EW (1980) Pathogens of acarina (mites). Bull World Health Organ 58(Suppl.):163-165

Dean RT (1977) Lysosomes. Edward Arnold

Dean RT (1979) Lysosomes and protein degradation. CIBA Found Symp 75:139-149

Desch CE, Andrews JRH, Arlian LG (1991) The digestive system of Sarcoptes scabei (L.): light and electron microscopy study. In: Dusbábek F, Bukva V (eds) Mod Acarol, vol 1. Akademia, Prague, pp 271-279

Devdhar MJ, Amoji SD (1978) On a new Actinocephalid Gregarine Contospora opalnia new genus new species from the gut of an arachnid Opalnia sp. Arch Protistenkd 120(1-2):182-189

Dinsdale D (1974) The digestive activity of a phthiracarid mite mesenteron. J Insect Physiol 20:2247-2260

Dinsdale D (1975) Excretion in an oribatid mite Phthiracarus sp. (Arachnida: Acari). J Zool Lond 177:225-231

Di Palma A (1996) Typhlodromus rheanoides Athias-Henriot e T.exhilaratusa Ragusa (Acari: Mesostigmata :Phytoseiidae):Osservazioni morfologiche, strutturali econsiderazioni funzionali. Tesi di Dottorato in 'Protezione dellaColture', Università degli Studi di Bari

Dolp RM (1970) Biochemical and physiological studies of certain ticks (Ixodoidea). Qualitative and quantitative studies of hemocytes. J Med Entomol 7(3):277-288

Douglas JR (1943) The internal anatomy of Dermacentor andersoni Stiles. Univ Calif Publ Entomol 7:205-272

Dubinin VB (1951) Federmilben (Analgesoidea). Teil I. Einführung in ihr Studium. Fauna SSSR Paukoobraznye (fauna der UdSSR, Spinnentiere) 6(5):1-363

Dufour ML (1856) Histoire anatomique et physiologique des scorpions. Mém pres par divers savans a l'acad de l'institut de France 14:561-657

Easwari Arama KG (1967) Anatomy and embryology of Eylais extendens (Mull.) (Family Eylidae: Hydracarina). Bull Dept Mar Biol Oceanogr Univ Kerala 3:75-130

Efremova LK (1967) Functioning of Malpighian tubules in the tick, Alveonasus lahorensis (Ixodoidea, Argasidae). Zool Zh 46(1):48-54 (in Russian)

Ehara S (1960) Comparative studies on the internal anatomy of three Japanese Trombidiforme Acarinids. J Fac Sci Hokkaido Univ Ser VI Zool 14(3):410-434

Eichenberger G (1970) Das Zentralnervensystem von Ornithodorus moubata und seine postembryonale Entwicklung. Acta Tropica 27:15-53

El Shoura SM (1988) Ultrastructural studies on the mid-gut epithelium and digestion in the female tick Argas (Persicargas) arboreus (Ixodoidea: Argasidae). Exp Appl Acarol 5(1-2):121-136

El Shoura SM (1989) Fine structure of the fat body in the female tick Ornithodoros (Pavlovskyella) erraticus (Ixodoidea: Argasidae). Exp Appl Acarol 7(3):245-249

Enigl M, Schausberger P (2007) Incidence of the endosymbionts Wolbachia, Cardinium and Spiroplasma in phytoseiid mites and associated prey. Exp Appl Acarol 42(7):75-85

Enigk K, Grittner I (1952) Excretion of ticks. Z Tropenmed Parasitol 4(1):77-94

Erban T, Hubert J (2010a) Comparative analyses of proteolytic activities in seven species of synanthropic acaridid mites. Arch Insect Biochem Physiol 75(3):187-206

Erban T, Hubert J (2010b) Determination of $\mathrm{pH}$ in regions of the mid-guts of acaridid mites. J Insect Sci 10(1):1-12

Erban T, Hubert J (2011) Visualization of protein digestion in the mid-gut of the acarid mite Lepidoglyphus destructor. Arch Insect Biochem Physiol 78(2):74-86

Erban T, Harant K, Hubert J (2017) Detailed two-dimensional gel proteomic mapping of the feces of the house dust mite Dermatophagoides pteronyssinus and comparison with D. farinae: reduced trypsin protease content in D. pteronyssinus and different isoforms. J Proteom 162:11-19. https://doi. org/10.1016/j.jprot.2017.04.021

Erhardt P, Voss G (1961) Die Carbohydrasen der Spinnmilbe Tetranychus urticae Koch. (Acari, Trombidiformes, Tetranychidae). Experientia 17:307 
Ehrnsberger R (1973) Untersuchungen zur Biologie und Anatomie der Milbenfamile Rhagidiidae. Dissertation, Kiel, Univ., Math.-Naturwiss. Fak

Evans GO, Till WM (1966) Studies on the British Dermanyssidae (Acari: Mesostigmata). Part II. Classification. Bull Brit Mus (NH) Zool 14(3):109-370

Filimonova SA (2001) The fine structure of the mid-gut of mite Myobia murismusculi. Tsitologila 43(5):425-431 (in Russian)

Filimonova SA (2008a) The fine structure of the mid-gut in the mite Anystis baccarum (L.) (Acari, Actinedida: Anystidae). Arthropod Struct Dev 37(4):299-309

Filimonova SA (2008b) Peculiarities of the internal anatomy in the mite family Syringophilidae by example of Syringophilopsis fringilla a parasite of the chaffinch. Parazitologiya 42(5):395-404 (in Russian)

Filimonova SA (2009) The ultrastructural investigation of the mid-gut in the quill mite Syringophilopsis fringilla (Acari, Trombidiformes: Syringophilidae). Arthropod Struct Dev 38(4):303-313

Filimonova SA (2013) Morphological aspects of blood digestion in a parasitic mite Bakericheyla chanayi. Arthropod Struct Dev 42(4):265-275

Flechtmann CHW, McMurtry JA (1992) Studies on how phytoseiid mites feed on spider mites and pollen. Int J Acarol 18:157-162

Frank HR (1938) Histologische Untersuchungen über die Verdauung bei Weberknechten. Zeitschr Morphol Ökol Tiere 33(1):51-164

Franta Z, Frantová H, Konvičhová J, Horn M, Sojka D, Mareš M, Kopáček P (2010) Dynamics of digestive proteolytic system during blood feeding of the hard tick Ixodes ricinus. Parasit Vectors 3:119

Frayha GJ, Dajani RM, Samia LH, Sweatman GK (1970) A dicarboxylic acid cycle in the tick Hyalomma dromedarii. J Parasitol 56(4)Sect.II:106

Frayha GJ, Dajani RM, Samia LH, Sweatman GK (1972) Dicarboxylic and amino-acid formation by nymphs of the tick Hyalomma dromedarii. Insect Biochem 2(5):78-86

Fried B, Boddorff JM (1975) Thin layer chromatographic analyses of neutral lipids of Unionicola campelomaicola (Hydracarina), a symbiont of Campeloma decisum (Gastropoda). Proc Pa Acad Sci 49(2):107-109

Friedhoff KT (1970) Microogranisms in engorged females of the tick Rhipicephalus bursa. J Parasitol 56(4 Sec.II Pt.1):111

Fuzita FJ, Pinkse MWH, Patane JSL, Juliano MA, Verhaert PDEM, Lopes AR (2016a) Biochemical, transcriptomic and proteomic analyses of digestion in the scorpion Tityus serrulatus: insights into function and evolution of digestion in an ancient arthropod. PLoS One 10(4):e0123841. https://doi. org/10.1371/journal.pone.0123841

Fuzita FJ, Pinkse MWH, Patane JSL, Verhaert PDEM, Lopes AR (2016b) High throughput techniques to reveal the molecular physiology and evolution of digestion in spiders. BMC Genom 17:716-735. https://doi.org/10.1186/s12864-016-3048-9

Gabbay S, Warburg MR (1976) Neurosecretory activity as related to feeding, mating, and oögenesis in the female cave tick, Ornithodoros tholozani. J Insect Physiol 22:1291-1301

Gabe M (1968) Caractères cytologiques et histochimiques de la glande coxale des Aranéides. Compte Rendus Hebd Seances Academie des Sciences Paris series D 266(11):1142-1144

Ganong WW (2005) Review of medical physiology. McGraw-Hill Medical, New York

Gasser R (1951) Zur Kenntnis der gemeinen Spinnmilbe Tetranychus urticae Koch. I. Mitteilung: Morphologie, Anatomie, Biologie und Ökologie. Bulletin de la Société Entomologique Suisse (Mitteilungen der Schweizerischen Entomologischen Gesellschaft) 24(3):217-262

Geczy AF, Naughton MA, Clegg JB, Hewetson RW (1971) Esterases and a carbohydrate-splitting enzyme in the saliva of the cattle tick, Boophilus microplus. J Parasitol 57(2):437-438

Gibson R, Barker PL (1979) The decapod hepatopancreas. Oceanogr Mar Biol Ann Rev 17:285-346

Gilbert D (1952) Studies of the histology of the mid-gut of the Chelonethi or Pseudoscorpiones. Q J Microsc Sci 93:31-45

Gooding RH (1972) Digestive processes of haematophagous insects. I. A literature review. Quaest Entomol 8:5-60

Gordon AW (1972) Animal physiology: principles and adaptations, 2nd edn. MacMillan, London

Gorgol VT (1991) Morphological features of gut ontogeny in the parasitic mite Varroa jacobsoni. Wiad Parazitologiya 37(1):95-97

Gorgol VT, Barabanova VV (1979) Activity of certain digestive enzymes as an indicator of carnivorousness of the mite Cheyletus eruditus (Cheyletidae, Trombidiformes). Dokl Akad Nauk Ukr SSR ser B geol Khim Biol Nauki 0(10):855-858

Gough JM, Kemp DH (1995) Acid phosphatase in mid-gut digestive cells in partially fed females of the cattle tick Boophilus microplus. J Parasitol 81(3):341-349 
Goyffon M, Martoja R (1983) Cytophysiological aspects of digestion and storage in the liver of a scorpion, Androctonus australis (Arachnida). Cell Tissue Res 228:661-675

Grandjean F (1939) Quelques genres d'Acariens appartenant au groupe des Endeostigmata (1 ${ }^{\text {ere }}$ série). Ann Sci Natur Ser II Zool et Biol Anim 2:1-222

Grandjean O (1984) Blood digestion in Ornithodorus moubata sensu stricto (Ixodoidea: Argasidae) females: 1. Biochemical changes in the mid-gut lumen and ultrastructure of the mid-gut cell, related to intracellular digestion. Acarologia 25(2):147-166

Grandjean O, Aeschlimann A (1973) Contribution to the study of digestion in ticks: histology and fine structure of the mid-gut epithelium of Ornithodoros moubata, Murray (Ixodoidea, Argasidae). Acta Trop 30(3): 193-212

Grasshoff M (1978) A model of the evolution of the main chelicerate groups. Symp Zool Soc Lond 42:273-284

Griffiths AB (1892) The physiology of the invertebrata. L Reeve and Co., London, p 443

Grigor'eva LA (2003) Morphofunctional changes in the mid-gut of tick females of the genus Ixodes (Acarina: Ixodidae) during and after feeding. Parazitologiya 37(3):169-176 (in Russian)

Grigor'eva LA (2004) Morphofunctional changes in the mid-gut of tick nymphs of the genus Ixodes (Acarina: Ixodidae) during and after feeding. Parazitologiya 38(3):219-224 (in Russian)

Grigor'eva LA (2005) Morphofunctional changes in the mid-gut of virgin tick females of the genus Ixodes (Acarina: Ixodidae) during and after feeding. Parazitologiya 39(4):265-269 (in Russian)

Grigor'eva LA (2006) Morphofunctional changes in the mid-gut of the Ixodes females (Acari: Ixodidae) during the immunizing feedings. Parazitologiya 40(4):363-370

Groepler W (1969) Feinstruktur der Coxalorgane bei der Gattung Ornithodorus. Zwiss Zool 178:235-275

Dr Gudden (1861) Beitrag zur Lehre von der Scabies. Würzburger medicinische Zeitschrift 2:301-319

Guirgis SS (1971) The subgenus Persicargas (Ixodoidea, Argasidae, Argas). 13. Histological studies on A.(P.) arboreus Kaiser, Hoogstraal \& Kohls. J Med Ent 8(6):648-667

Guiyesse A (1908) Etude des organes digestifs chez le scorpion. Archiv Anatomie Microscopie Paris 10:123-139

Haarløv N, Bresciani J (1972) Light and electron microscopical studies on the alimentary system of an oribatid mite, Nothrus palustris C.L.Koch, 1840. In: Proceedings of the XIII international congress ENT, Moscow 2-9 Aug 1968, vol III, pp 346-348

Hafiz A (1935) Embryological development of Cheyletus eruditus. Proc R Soc Lond Ser B 7:174-201

Hajjar NP (1972) Biochemical and physiological studies of certain ticks (Ixodoidea). Fatty acid composition of lipids and free fatty acid fractions of hemolymph and gut and molting fluids of nymphal and female Hyalomma (H.) dromedarii Koch and H. (H.) anatolicum excavatum Koch (Ixodidae). J Med ENT 9(6):551-557

Haller G (1880) Zur Kenntnis der Tyroglyphen und Verwandten. Zeitschrift für wiss Zool 34(2):255-295

Hamburger Cl (1916-1917) Zur Kenntnis des Mitteldarmes der Spinnen. Zool Anz 438:39-45

Hamdy BH (1973) Biochemical and physiological studies of certain ticks (Ixodoidea). Cycle of nitrogenous excretion in Argas (Persicargas) arboreus Kaiser, Hoogstraal \& Kohls (Argasidae). J Med ENT 10(1):53-57

Hamdy BH (1977) Biochemical and physiological studies of certain ticks (Ixodoidea). Excretion during ixodid feeding. J Med Entomol 14(1):15-18

Hamilton KA, Nisbet AJ, Lehane MJ, Taylor MA, Billingsley PF (2003) A physiological and biochemical model for digestion in the ectoparasitic mite, Psoroptes ovis (Acari: Psoroptidae). Int J Parasitol 33(8):773-785

Harrison FW, Foelix RF (1999) Microscopic anatomy of invertebrates. Chelicerate Arthropoda, vols 8A. Wiley, New York

Hartmann D, Šíma R, Konvičková Jitka, Perner J, Kopáček P, Sojka D (2018) Multiple legumain isoenzymes in ticks. Int J Parasitol 48(2):167-178

Hassan AS (1928) The biology of the Eriophyidae with special reference to Eriophyes tristriatus (Nalepa). University of California publications in entomology 4(11):341-394

Hecker H, Rudin W (1981) Morphometric parameters of the mid gut cells of Aedes aegypti Insecta Diptera under various conditions. Cell Tissue Res 219(3):619-627

Hecker H, Aeschlimann A, Burkhardt MJ (1968) Contribution to the knowledge of symbiotes in Ornithodorus moubata Ixodoidea. Electron microscopic study. Acta Trop 25(3):256-262

Heethoff M, Norton RA (2009) Role of musculature during defecation in a particle-feeding arachnid, Archegozetes longisetosus (Acari, Oribatida). J Morphol 270:1-13. https://doi.org/10.1002/jmor.10667

Hefnawy T (1972a) Biochemical and Physiological Studies of Certain Ticks (Ixodoidea). Hemolymph Volume Determined by Isotope and Dye Dilution during the Gonotrophic Cycle of Argas 
(Persicargas) persicus (Oken) and A. (P.) arboreus Kaiser, Hoogstraal, and Kohls (Argasidae). J Parasitol 58(2):358-364

Hefnawy T (1972b) Biochemical and Physiological Studies of Certain Ticks (Ixodoidea). Osmotic Pressure of Hemolymph and Gut and Coxal Fluids during the Gonotrophic Cycle of Argas (Persicargas) persicus (Oken) and A. (P.) arboreus Kaiser, Hoogstraal, and Kohls (Argasidae). J Parasitol 58(6):1197-1200

Hefnawy T, Dimos JP (1974) Biochemical and physiological studies of certain ticks (Ixodoidea): specific gravity of hemolymph and coxal and gut fluids of Argas (Persicargas) persicus and Argas (Persicargas) arboreus (Argasidae). Exp Parasitol 36(1):1-5

Heilesen B (1946) Studies on Acarus scabiei and scabies. Acta Dermato Venereologica 26(Suppl. 14):1-370

Heller C (1858) Zur Anatomie von Argas persicus. Sitzungsberichte der kaiserl Akademie Wien MathemNaturwiss Klasse 30(16):297-326 (4 plates)

Henking H (1882) Beiträge zur Anatomie, Entwicklungsgeschichte und Biologie von Trombididum fuliginosum, Herm Zeitschr f wissenschaft Zool 37:553-663 (6 plates)

Herman WS, Preus DM (1972) Ultrastructure of the hepatopancreas and associated tissues of the chelicerate arthropod Limulus polyphemus. Zeitschrift Zellforsch Mikrosk Anat 134(2):255-271

Hickman VV (1942) On some new Hadrotarsidae (Araneae) with notes on their internal anatomy. In: Papers and Proceedings of the royal society of Tasmania, pp 147-160 (5 plates)

Hirschmann W (1969)Gangsystematik der Parasitiformes. Acarologie Schriftenreihe für vergleichende Milbenkünde. Hirschmann-Verlag, Fürth/Bayern. Folge 13:30-55 (13 Tafel)

Hoebel-Mävers M (1967) Funktionsanatomische Untersuchungen am Verdauungstrakt der Hornmilbeln (Oribatei). Zur Erlangung des Grades eines Doktors der Naturwissenschaften Dr. rer. nat. genehmigte Dissertation Von der Naturwissenschaftlich-Philosophischen Fakultät der Technischen Hochschule Carlo-Wilhelmina zu Braunschweig aus Adenstedt Kreis Alfeld, pp 1-46 (71 Figs)

Hoese B (1981) Morphologie und Funktion des Wasserleitungssystems der terrestrischen Isopoden (Crustacea, Isopoda, Oniscoidea). Zoomorphology 98:135-167

Holt DC, Burgess ST, Reynolds SL, Mahmood W, Fischer K (2013) Intestinal proteases of free-living and parasitic astigmatic mites. Cell Tissue Res 351(2):339-352

Holter H (1961) How things get into cells. Sci Am 205(3):167-183

Hoogstraal H (1983) Book review: physiology of ticks. Insect Sci Appl 4(4):417-420

Horn M, Nussbaumerová M, Sanda M, Kovárová Z, Srba J, Franta Z, Sojka D, Bogyo M, Caffrey CR, Kopáček P, Mareš M (2009) Hemoglobin digestion in blood feeding ticks: mapping a multipeptidase pathway by functional proteomics. Chem Biol 16(10):1053-1063

Hoy MA, Jeyaprakash A (2008) Symbionts, including pathogens, of the predatory mite Metaseiulus occidentalis: current and future analysis methods. Exp Appl Acarol 46(1-4):329-347

Hubert J, Šustr V (2001) The effect of starvation on the metabolic rate and microanatomy of Galumna elimata (Acari: Oribatida). Eur J Entomol 98:265-275

Huger AM, Krieg A (1967) New aspects of the mode of reproduction of Rickettsiella organisms in insects. J Invertebr Pathol 9:442-5

Hughes TE (1950a) The physiology of the alimentary canal of Tyroglyphus farinae. Q J Microsc Sci 91(1):45-61

Hughes TE (1950b) Some stages of Litomosoides carinii in Liponyssus bacoti. Ann Trop Med Parasitol 44:285-290

Hughes TE (1952) The morphology of the gut in Bdelonyssus bacoti (Hirst, 1913) Fonseca, 1941. Ann Trop Med Parasitol 46(1):54-60

Hughes TE (1954a) The internal anatomy of the mite Listrophorus leukarti (Pagenstecher, 1861). Proc Zool Soc Lond 124(2):239-256. https://doi.org/10.1111/j.1469-7998.1954.tb07781.x

Hughes TE (1954b) Some histological changes which occur in the gut epithelium of Ixodes ricinus females during gorging and up to oviposition. Ann Trop Med Parasitol 48(4):397-404

Hughes TE (1959) Mites or the Acari. University of London. The Athlone Press, London

Hughes TE, Hughes AM (1939) The internal anatomy and post-embryonic development of Glycyphagus domesticus De Geer. Proc Zool Soc Lond Ser B 108(4):715-733

Hugon J, Borgers M (1968) Fine structural localization of acid and alkaline phosphatase activities in the absorbing cells of the duodenum of rodents. Histochemie 12:42-66

Humiczewska M, Mielnicka ME (1983) Histoenzymatic investigations of digestion and catabolic processes in Tetranychus urticae (Acarida, Tetranychidae). Folia Biol (Cracow) 31(4):419-426

Ishidoh K, Kominami E (2002) Processing and activation of lysosomal proteinases. Biol Chem 383(12):1827-1831

Jakeman LAR (1961) The internal anatomy of the spiny rat mite, Echinolaelaps echidninus (Berlese). J Parasitol 47:328-349 
Jakeman LA, Strandtmann RW (1960) The internal anatomy of the female spiny rat mite, Echinolaelaps echidninus. J Parasitol 46(5/2):20

Jalil M (1969) Internal anatomy of the developmental stages of the two-spotted spider mite. Ann ENT Soc Am 62:247-249

Jaschke W (1933) Beiträge zur Kenntnis der Symbiontischen Einrichtungen bei Hirudineen und Ixodiden. Zeitschrift für wiss Biol Abt F Zeitschrift Parasitend 5(3-4):515-541

Jeffers LA, Roe RM (2008) The movement of proteins across the insect and tick digestive system. J Insect Physiol 54(2):319-332

Jones BM (1950) The penetration of the host tissue by the harvest mite, Trombicula autumnalis Shaw. Parasitology 40:247-259

Jones PE (1975) Notes on the predators and prey of British Pseudoscorpions. Bull Br Arachnol Soc 3(4):104-105

Jordan H (1912) Die "Leberfrage" bei den wirbellosen Tieren. Zool Jahrbuch Supplementband 15 Abt 3:49-58

Kaestner A (1969a) Lehrbuch der Speziellen Zoologie. Band I: Wirbellose. 1. Teil Protozoa, Mesozoa, Parazoa, Coelenterata, Prostomia ohne Mandibulata. VEB Gustav Fischer Verlag, Jena

Kaestner A (1969b) Invertebrate zoology: arthropod relatives, Chelicerata, Myriapoda, vol 2 (transl) Levi HW, Levi LR. Wiley, New York

Kal'vish TK, Sharapov VM, Davydova MS (1978) Entomophthorosis of gamasid mites (Acarina, Gamasoidea). Parazitologiya 12(2):189-190 (in Russian)

Karpelles L (1893) Zur Anatomie von Bdella arenaria (Kramer). Verhandl d k k zool-bot Ges, Z B Ges B 43:421-430 (2 plates)

Kästner A (1940) Arachnida. In: Kükenthal W (ed.) Handbuch der Zoologie vol 8; part (1):97-116; part (2):1-656; part (3):1-160. Walter de Gruyter \& Co., Berlin

Kanungo K (1964) Disappearance of blood from the gut of engorged Echinolaelaps echidninus (Acarina: Laelaptidae). Ann Entomol Soc Am 57(4):427-428

Kanungo K, Naegele JA (1964) The haemocytes of the acarid mite Caloglyphus berlesei (Mich. 1903). J Insect Physiol 10:651-655

Kanwar KC, Malik L (1966) Mechanism of food transport in cattle tick, Hyalomma aegyptium. Res Bull (N.S.) Punjab Univ 17 (3-4):423-424

Karg W (1971) Die freilebenden Gamasina (Gamasides), Raumilben. Die Tierwelt Deutschlands und der angrenzenden Meeresteile nach ihren Merkmalen und nach ihrer Lebensweise: Acari (Acarina), Milben: Unterordnung Anactinochaeta (Parasitiformes). Teil 59:1-475

Khalil GM (1971) Biochemical and physiological studies of certain ticks (Ixodoidea). Incorporation of tritiated tyrosine in the digestive system of Nymphal Argas (Persicargas) arboreus (Argasidae). Ann Entomol Soc Am 64(5):1149-1154

Khizhinsky PG (1968) Consumption of reserve nutritive substances during the lifespan of unfed female Ixodes persulcatus P.Sch. Med Parazit, Moskva 37(3):291-297

Khomyakov IP (1967) On the occurrence of some species of burrow Gamasidae hematophages in soil. Zool Zh 46:345-350 (in Russian)

Kilby BA (1963) The biochemistry of the insect fat body. Adv Insect Physiol 1:111-174. https://doi. org/10.1016/S0065-2806(08)60175-7

King LAL (1913) Notes on the habits and characteristics of some littoral mites of Millport. Proc R Phys Soc 19(17):129-141

Kirkup L, Sutherland J (1988) Curve stripping and nonlinear fitting of polyexponential functions to data using a microcomputer. Comput Phys 2:64-68. https://doi.org/10.1063/1.168313

Kitaoka S (1961a) Physiological and ecological studies on some ticks. IV. Physiological stage and lipid deposit during the blood sucking process in the tick. Q Natl Inst Anim Health Tokyo 1(2):85-95

Kitaoka S (1961b) Physiological and ecological studies on some ticks. V. Nitrogen and iron excretion and amount of blood meal ingested during the blood sucking process in the tick. Q Natl Inst Anim Health Tokyo 1(2):96-104

Kitaoka S (1961c) Physiological and ecological studies on some ticks. VI. Rate of digestion of blood meal and nitrogen, iron and sterol economy during the ovipositing process in the tick. Q Natl Inst Anim Health Tokyo 1(2):105-112

Klann AE (2009) Histologie und Ultrastruktur der Solifugen. Vergleichende Studien an Organsystemen der Solifugen (Arachnida, Solifugae) mit Schwerpunkt auf funktionellen Analysen und phylogenetischen Interpretationen. Inauguraldissertation zur Erlangung des akademischen Grades doctor rerum naturalium (Dr. rer. nat.) an der Mathematisch-Naturwissenschaftlichen Fakultät der Ernst-Moritz-ArndtUniversität Greifswald 
Klumpp W (1954) Embryologie und Histologie der Bienenmilbe Acarapis woodi Rennie 1921. Z f Parasitenkunde 16(5-6):407-442

Knülle W (1967) Significance of fluctuating humidities and frequency of blood meals on the survival of the spiny rat mite, Echinolaelaps echidninus (Berlese). J Med ENT 4(3):322-325

Kobert R (1903) Über die Enzyme wirbelloser Tiere. Pflügers Arch Physiol 99:116-186

Kolodochoka LA, Lysaya EA (1976) Survival of hungry predatory mites phytoseiid mites Phytoseiulus persimilis, Amblyseius andersoni and Amblyseius reductus (Parasitiformes, Phytoseiidae). Vestnik Zoologii 0(3):88-90 (in Russian)

Kostenko SM, Akimov IA, Starovir IS (1978) The discovery of Gregarinidae in some Acaroid mites. Vestnik Zoologii 0(2):61-64 (in Russian)

Kötter C (1978) Ein Beitrag zur Stoffwechselphysiologie von Tetranychus urticae Koch (Acari, Tetranychidae). Zeitschrift für angewandte Entomologie 86(4):337-348

Kramer P (1885) Ueber Halarachne halichoeri. Allm Z Naturwis 58(4):46-74

Kramer P (1886) Das Herz der Gamasiden. Zool Anz Leipzig 9:553-554

Kreier JP (1977a) (ed) Parasitic protozoa. Volume I taxonomy, kinetoplastid and flagellates of fish. Academic Press, New York

Kreier JP (1977b) (ed) Parasitic protozoa. Volume III. Gregarines, haemogregarines, coccidia, plasmodia, and haemoproteids. Academic Press, New York

Krijgsman BJ (1954) Die Resorption von physiologisch wichtigen Stoffen im Magendarmkanal bei Vertebraten und Invertebraten. Tabulae Biologicae, digestion no 16 21(2):203-239

Kroman RA (1979) The correlation of the life cycle of a trombiculid mite and its fungal symbiont. In: Proceedings of the 4th international congress of acarology, 1974, pp 483-491

Kroman RA, Loomis RB, Swater FE, Omieczynski DT (1969) The presences of yeastlike cells of probable symbiotic natures in trombiculid mites. In: Proceedings of the 2 nd international congress of acarology, 1967, pp 309-315

Krüger P (1934) A. Verdauung. 1. Verdauung und Resorption der wirbellosen Tiere. Handbuch Biochemie d Menschen und Tiere 2:415-430

Krunkenberg CFrW (1880) Vergleichende physiologische Studien, 2nd edn. Experim Untersuchungen, Heidelberg

Kühnelt W (1961) Soil biology. Faber \& Faber, Fabriano

Kullmann E, Kloft W (1968) Traceruntersuchungen zur Regurgitationsffitterung bei Spinnen (Araneae, Theridiidae). Zool Anz (Suppl) 32:487-497

Kuo JS, Nesbitt HHJ (1970) The internal morphology and histology of adult Caloglyphus mycophagus. Can J Zool 48:505-518

Kuo JS, Nesbitt HHJ (1971) Internal morphology of the hypopus of Caloglyphus mycophagus. Acarologia 13(1):156-170

Lagutenko YuP (1962) Microscopic anatomy of certain systems of organs of the fowl tick Dermanyssus gallinae (Gamasoidea, Dermanyssidae). Zool Zh 41(6):840-853 (in Russian)

Lagutenko YuP (1963) Functional and histological features of the gonotrophic cycle of the chicken mite Dermanyssus gallinae (Gamasoidea, Dermanyssidae). Med Parazitol (Mosk) 32(3):313-319 (in Russian)

Lake BD, Ellis RB (1976) What do you think you are quantifying? An appraisal of histochemical methods in the measurement of the activities of lysosomal enzymes. Histochem J 8:357-366

Lang A (1891) Text-book of comparative anatomy. (transl) Bernard HM, Bernard M. Part I. Macmillan and Co, London, pp 509-543

Langenscheidt M (1958) Embryologische, Morphologische und Histologische Untersuchungen an Knemidocoptes mutans (Robin et Lanquetin). Z f Parasitenkunde 18:349-385

Langley PA (1976) Initiation and regulation of ingestion by hematophagous arthropods. J Med Entomol 13(2):121-130

Lankester ER (1904) The structure and classification of the Arachnida. Q J Microsc Sci New Ser 48(189):165-269

Lara FA, Lins U, Paiva-Silva G, Almeida IC, Braga CM, Miguens FC, Oliveira PL, Dansa-Petreski M (2003) A new intracellular pathway of haem detoxification in the mid-gut of the cattle tick Boophilus microplus: aggregation inside a specialized organelle, the hemosome. J Exp Biol 206(10):1707-1715

Lara FA, Lins U, Bechara GH, Oliveira PL (2005) Tracing heme in a living cell: hemoglobin degradation and heme traffic in digest cells of the cattle tick Boophilus microplus. J Exp Biol 208(16):3093-3101

Leake LD (1975) Comparative histology. An introduction to the microscopic structure of animals. Academic Press, London, p 738

Lees AD (1964) The effect of ageing and locomotor activity on the water transport mechanisms of ticks. Acarologia hs (C. R $1^{\text {er }}$ Congrès Int d'Acarologie. Fort Collins, Col 1963, pp 315-323 
Legendre R (1967) Morphologie und Entwicklungsgeschichte der Cheliceraten. Entwicklung, Anatomie und Histologie der Milben (Acari). Fortschritte der Zoologie 18:207-222

Legendre R (1968) La nomenclature anatomique chez les Acariens. Acarologia 10(3):413-417

Lekimme M, Leclercq-Smekens M, Devignon C, Leclipteux T, Pounnay Y, Losson B (2005) Ultrastructural morphology of male ands female genital tracts of Psoroptes spp. (Acari: Astigmata: Psoroptidae). Exp Appl Acarol 36:305-324

Lindeman RL (1942) The trophic-dynamic aspect of ecology. Ecology 23:399-418. https://doi. org/10.2307/1930126

Lindquist EE (1984) Current theories on the evolution of major groups of Acari and on their relationships with other groups of Arachnida, with consequent implications for their classification. In: Griffiths DA, Bowman CE (eds) Acarology VI, vol 1. Wiley, New York, pp 28-62

Lister A, Block W, Usher MB (1988) Arthropod predation in an antarctic terrestrial community. J Anim Ecol 57:957-971

Liyou N, Hamilton S, Watters DJ, Tellman R, Willadsen P (1996) Endocytosis by digest cells of the cattle tick Boophilus microplus: regulation by protein kinase C. Insect Biochem Mol Biol 26(2):147-154

Lombardini G (1942) Contributo alla conoscenza della morfologia dei Demodicidae. Chiave analitica del genere "Demodex" Owen. Redia 28:89-102 (1 plate)

Lönnfors F (1930) Beiträge zur Morphologie der Analginen. Acta Zoo Fenn 8:1-81

Ludwig M, Alberti G (1988a) Mineral congregations, "Spherites" in the mid-gut gland of Coelotes terrestris (Araneae): structure, composition and function. Protoplasma 143:43-50

Ludwig M, Alberti G (1988b) Digestion in spiders-histology and fine-structure of the midgut gland of Coelotes terrestris (Agelenidae). J Submicrosc Cytol Pathol 20(4):709-718

Lundblad O (1930) Über die Anatomie von Arrhenurus mediorotundatus und die Hautdrüsen der Arrhenurus-Arten. Zeitschrift für Morphologie und Ökologie der Tiere Abt A Band 17(1/2):302-338

Luxton M (1966) Laboratory studies on the feeding habits of saltmarsh Acarina, with notes on their behaviour. Acarologia 8(I):163-175

Maathai WMJM (1977) An electron microscope study of the type (C) and (D) cells in adult unfed brown ear tick, Rhipicephalus appendiculatus (Neumann 1901) fed as nymphs on animals suffering from east coast fever. Bull Anim Health Prod Afr 25(4):415-420

MacLeod J (1884a) Communication préliminaire relative à l'anatomie des Acariens. Bull Acad Belg Cl Sci Ser 3 Acad R Sci Lett Beaux-arts beig Bul 7:253-259

Macleod J (1884b) La structure de l'intestin anterieur des Arachnides. Bull Acad R Sci Belg Ser 3 $8(53): 377-396$

Mansour-Bek JJ (1954) The digestive enzymes in Invertebrata and Protochordata Tabulae Biologicae-21 digestion part 3(no 24):75-382

Markwardt F, Landmann (1961) Über einen Hemmstoff der Thrombokinase aus der Lederzecke Ornithodorus moubata. Naturwissenschaften 48:433

Maroun NA (1972) Biochemical and physiological studies of certain ticks (Ixodoidea). Lipids in eggs, larvae, and biological fluids of nymphal and adult argas (Persicargas) persicus (Oken) and A. (P.) arboreus Kaiser, Hoogstraal \& Kohls (Argasidae). J Med Entomol 9(2):161-167. https://doi. org/10.1093/jmedent/9.2.161

Maroun NA, Kamel KA (1973) Biochemical and physiological studies of certain ticks (Ixodoidea). Fatty acid composition of the female, eggs, and larva of Dermacentor andersoni (Stiles) (Ixodidae). J Med Entomol 10(4):400-405. https://doi.org/10.1093/jmedent/10.4.400

Maroun NA, Kamel KA (1976) Biochemical and physiological studies of certain ticks (Ixodoidea). Absence of sterol biosynthesis in Dermacentor andersoni Stiles (Acarina: Ixodidae). J med ENT 13(2):219-220

Mathers NF (1973) A comparative histochemical survey of enzymes associated with the processes of digestion in Ostrea edulis and Crassostrea angulata (Mollusca: Bivalvia). J Zool Soc Lond 169:169-179

Mathieson BR, Lehane MJ (2002) Ultrastructure of the alimentary canal of the sheep scab mite, Psoroptes ovis (Acari: Psoroptidae). Vet Parasitol 104(2):151-166

Matsumoto K (1974) Digestive enzymes of ticks. Jpn J Sanit Zool 24(4):294

McBrayer JF, Reichle DE (1971) Trophic structure and feeding rates of forest soil invertebrate populations. Oikos 22:381-388

McCall H (1976) Stimulation of acid phosphatase (Acph) activity in isolated salivary glands of Rhipicephalus appendiculatus. Trans R Soc Trop Med Hyg 70(4):286

McEnroe WD (1936a) Esterases in the two-spotted spider mite. Adv Acarol 1:214-224

McEnroe WD (1963b) The role of the digestive system in the water balance of the two-spotted spider mite. Adv Acarol 1:225-231 
McEnroe WD (1970) Fat in the mid-gut walls of the overwintering two-spotted spider mite Tetranychus urticae. Ann ENT Soc Am 63:912-913

McKinley DJ (1963) The morphology and biology of Haemolaelaps casalis Berlese (Acarina: Mesostigmata). Ann Mag Nat Hist Ser 13(6):65-76

Mégnin P (1876) Mémoire sur l'organisation et la distribution zoologique des acariens de la famille des Gamasides. Journ de l'Anat et de la Physiol 12:288-336

Mégnin P (1886) Nouvelle etude anatomique et physiologique sur les Glyciphages. C R Acad Sci 103:1276-1278

Mehrota KN (1961) Carbohydrate metabolism in the two-spotted spider mite Tetranychus telarius L. 1. Hexose monophosphate cycle. Comp Biochem Physiol 3:184-198

Mehrota KN (1962) Malic dehydrogenase in the two spotted spider mite, Tetranychus telarius (L.). Can J Biochem Physiol 40:1529-1533

Mehrota KN (1963a) Carbohydrate metabolism in the two-spotted spider mite Tetranychus telarius L. 2. Embden-Meyerhof pathway. Can J Biochem Physiol 41:12595-1602

Mehrota KN (1963b) Carbohydrate metabolism in the two spotted spider mite. Adv Acarol 1:232-237

Men YT (1959) Concerning the feeding of the mite Haemolaelaps casalis (Gamasoidea, Parasitiformes). Medskaya Parazitologiya 28:603-609 (in Russian)

Michael AD (1883) I. Observations on the anatomy of the Oribatidae. J R Microsc Soc Ser II 3(1):1-25 (2 plates)

Michael AD (1884) British Oribatidae, vol 1. Ray Society, London, p 333

Michael AD (1889) Observations on the special internal anatomy of Uropoda krameri. J R Microsc Soc Lond 22:1-15 (1 plate)

Michael AD (1892) On the variations in the internal anatomy of the Gamasinae, especially in that of the genital organs, and their mode of coition. Trans Linn Soc Lond Ser 2 Zool 5(4):281-325 (4 plates)

Michael AD (1894) Notes on the Uropodinae. J R Microsc Soc 27(1):289-319

Michael AD (1895) A study of the internal anatomy of Thyas petrophilus an unrecorded Hydrachnid found in Cornwall. Proc Zool Soc Lond 12-13:174-209

Michael AD (1896a) The internal anatomy of Bdella. Trans Linn Soc Lond Zool Ser 2 6(7):477-528

Michael AD (1896b) On some undescribed Acari of the genus Glyciphagus, found in Moles' Nests. Zool J Linn Soc 19:269-284

Michael AD (1901) British Tyroglyphidae Volume. 1. Ray Society, London

Michael AD (1903) British Tyroglyphidae, vol 2. Ray Society, London

Micherdziński W (1969) Die Familie Parasitidae Oudemans 1901 (Acarina, Mesostigmata). Zaklad Zoologii Systematycznej Polskiej Akademii Nauk Państwowe Wydawnictwo Naukowe, Kraków 1-690

Millot J (1926) Contribution a l'histophysiologie des Araneides. Suppléments au Bulletin Biologique de France et de Belgique 8:1-238 (5 plates)

Millot J (1931a) Anatomie comparée de l'intestin moyen céphalothoracique chez les Araignées vrais. C R Acad Sci Paris 192:375-377

Millot J (1931b) Les diverticules intestinaux du cepahalothorax chez les Araignées vraies. Zeitschrift fur Morphol und Öekol tiere 21:740-764

Millot J (1942) Sur l'anatomie et l'histophysiologie de Koenenia mirabilis grassi (Arachnida Palpigradi). Rev Franc Entom Paris 9(2):33-51

Millot J (1943) Notes complémentaires sur l'anatomie, l'histologie et la répartition géographique en France de Koenenia mirabilis grassi. Rev Franc Entom Paris 9(3-4):131-137

Millot J (1945a) La constitution de l'abdomen des Ricinulei. Bull Soc Entomol Fr 50:72-74

Millot J (1945b) L'anatomie interne des Ricinulei (Arachnides). Ann Sci Nat Zoo et Bio an Paris Ser 11(7):1-29

Mitchell R (1955) Anatomy, life history and evolution of the mites parasitizing fresh-water mussels. Misc Publ Mus Zool Univ Michigan 89:1-28

Mitchell R (1964) The anatomy of an adult chigger mite Blankaartia acuscutellaris (Walch). J Morphol 114(3):373-392

Mitchell R (1970) The evolution of a blind gut in trombiculid mites. J Nat Hist 4:221-229

Mitchell R, Nadchatram M (1969) Schizeckenosy: the substitute for defecation in chigger mites. J Nat Hist 3:121-124

Mommsen TP (1978a) Digestive enzymes of a spider (Tegenaria atrica Koch) - I. General remarks, digestion of proteins. Comp Biochem Physiol A Comp Physiol 60(4):365-370

Mommsen TP (1978b) Digestive enzymes of a spider (Tegenaria atrica Koch)—III. Esterases, phosphatases, nucleases. Comp Biochem Physiol A 60:377-382

Moorehouse DE (1975) Studies on the feeding of larval Argas persicus Oken. Zeitschrift für Parasitenkunde 48(1):65-71 
Moreti R, Perrella NN, Lopes AR (2013) Carbohydrate digestion in ticks and a digestive $\alpha$-L-fucosidase. J Insect Physiol 59(10):1069-75

Morton J (1979) Guts. Hodder, 2nd revised edition

Moss WW (1962) Studies on the morphology of the trombidiid mite Allothrombium lerouxi Moss (Acari). Acarologia 4(3):313-345

Mothes U, Seitz K-A (1980) Licht- und elektronenmikroskopische Untersuchungen zur Funktions-morphologie von Tetranychus urticae (Acari, Tetranychidae). I Excretionssysteme. Zool Jahrb, Abt Anat Ontog Tiere 104(4):500-529

Mothes U, Seitz K-A (1981a) Licht- und elektronenmikroskopische Untersuchungen zur Funktions-morphologie von Tetranychus urticae (Acari, Tetranychidae). II Weibliches Geschlechtssytem und Oogenes. Zool Jahrb, Abt Anat Ontog Tiere 105(1):106-134

Mothes U, Seitz K-A (1981b) Functional microscopic anatomy of the digestive system of Tetranychus urticae (Acari, Tetranychidae). Acarologia 22(3):257-270

Mothes-Wagner U (1985) Fine structure of the 'hind-gut' of the two-spotted spider mite, Tetranychus urticae, with special reference to origin and function. Exp Appl Acarol 1(3):253-272

Moura-Martiniano NO, Machado-Ferreira E, Gazêta GS, Soares CAG (2017) Relative transcription of autophagy-related genes in Amblyomma sculptum and Rhipicephalus microplus ticks. Exp Appl Acarol 73:401-428

Mudrow E (1933) Über die Intrazellulären Symbionten der Zecken. Zeitschrift für Biol Abt F Zeitschrift für Parasitenkunde 5(1):138-183

Müller J (1828) Beiträge zur anatomie des Scorpions. Meckel's Archiv für Anatomie und Physiologie 13:29-70

Müller KH (1939) Zur Biologie der Taubenzecke Argas columbarum. Inaugural Dissertation zur Erlangung des Grades eines Doktors der veterinärmedizin aus dem Institut für veterinärmedizinische Parasitologie und Zoologie der Friedrich-Wilhelms Universität Berlin. JCF Pickenhahn, Univ California

Murray RA, Solomon KG (1978) A rapid technique for analysing diets of invertebrate predators by electrophoresis. Ann Appl Biol 90:7-10

Nakamura K (1972) The ingestion in wolf spiders. Part 2. The expression of degree of hunger and amount of ingestion in relation to spider hunger. Res Popul Ecol (Kyoto) 14(1):82-96

Nalepa A (1884) Die Anatomie der Tyroglyphen. I. Abteilung. Sitzungsberichte Ber d kais Akad Wissenschaften zu Wien, Math-naturw Klasse Abt. I, Bd 90(1):197-228

Nalepa A (1885) Die Anatomie der Tyroglyphen. II. Abteilung. Sitzungsberichte Ber d kais Akad Wissenschaften zu Wien, Math-naturw Klasse Abt. II, Bd 92(1):116-167

Nalepa A (1887) Die Anatomie der Phytopten. Sitzungsberichte Ber d kais Akad Wissenschaften zu Wien, Math-naturw Klasse 96:115-165

Neri F (1896) Contribuzione all'anatomie del Gamasidi. Anatomia del Dermanyssus gallinae Redi. Atti Soc Tosc Sci Nat Pisa Proc Verb 10:126-138

Neumann KW (1941) Beiträge zur Anatomie und Histologie von Parasitus kempersi OUDMS (Parasitidae) Zeitschrift für Morphologie und Ökologie der Tiere 37(4):1-76 (613-683)

Nevin FR (1935) Anatomy of Cnemidocoptes mutans (R. \& L.), the scaly-leg mite of poultry. Ann Entomol Soc Am 28(3):338-361

Newstead R, Duvall HM (1918) Bionomic, morphological and economic report on the acarids of stored grain and flour. Committee Royal Society. Reports of the grain pests (War) no. 2:1-41

Nisbet AJ, Billingsley PF (2000) A comparative survey of the hydrolytic enzymes of ectoparasitic and freeliving mites. Int J Parasitol 30:19-27

Nordenskiöld E(1898) Beiträge zur Kenntnis der Morphologie und Systematik der Hydrachniden. Acta Societatis scientiarum Fennicae 24(5):1-75 (2 plates)

Nordenskiöld E (1900) Beiträge zur Kenntnis der Anatomie von Norneria Gigas R. Can Acta Societatis Scientiarum Fennicae 26(6):1-23

Nordenskiöld E (1905) Zur Anatomie und Histologie von Ixodes reduvius II. Zoolog Anz 28:478-485

Nordenskiöld E (1906) Zur Anatomie und Histologie von Ixodes reduvius III. Zoolog Anz 30:118-125

Nordenskiöld E (1908) Zur Anatomie und Histologie von Ixodes reduvius I. Zool Jahrb Abt f Anat 25(4):637-674

Nordenskiöld E (1909) Zur Anatomie und Histologie von Ixodes reduvius. II. Zool Jahrb Abt f Anat 27:449-464

Nordenskiöld E(1911) Zur Anatomie und Histologie von Ixodes reduvius. III. Zool Jahrb, Abt f Anat 32(1):77-106

Norval RAI, Capitini LA (1974) The rate of feeding and spermatogenesis in Amblyomma hebraeum Koch. J Entomol Soc S Afr 37(2):393-401 
Nuttal GHF (1909) The presence of anticoagulin in the salivary glands of Argas persicus. Camb Proc Philos Soc 15:53

Nuttal GHF, Strickland C (1908) On the presence of an anticoagulin in the salivary glands and intestines of Argas persicus. Parasitology 1(4):302-310

Nuzzaci G (1976) Contributo alla Conoscenza dell'Anatomia degli Acari Eriofidi. Entomologia (Bari) 12:21-56

Nuzzaci G (1979) A study of the internal anatomy of Eriophyes canestrini Nal. In: Proceedings of the 4th international congress of acarology, 1974, pp 725-727

Nuzzaci G, Di Palm A, Aldini P (2001) Functional morphology and fine structure of the female genital system in Typhlodromus spp. (Acari: Phytoseiidae). In: Halliday RB, Walter DE, Proctor HC, Norton RA, Colloff M J (eds) Acarology: Proceedings of the 10th international congress. CSIRO Publishing, Melbourne, pp 196-202

Obata Y (1954) Anatomical studies on Trombicula akamushi (Brumpt), the vector of Tsutsugamushi disease. Med Entomol Zool 5(3-4):111-145

Obenchain FD, Oliver JH Jr (1973) A qualitative analysis of the form, function and interrelationships of fat body and associated tissues in adult ticks (Acari-Ixodoidea). J Exp Zool 186(3):217-236

Obenchain FD, Oliver JH Jr (1976) The heart and arterial circulatory system of ticks (Acari: Ixodoidea). J Arachnol 3:57-74

Oboussier H (1939) Beiträge zur Biologie und Anatomie der Wohnungsmilben. Zeitschrift für Angewandte Entomologie 26:253-296

Occhipinti A, Maffei ME (2013) Chlorophyll and its degradation products in the two-spotted spider mite, Tetranychus urticae: observations using epifluorescence and confocal laser scanning microscopy. Exp Appl Acarol 61:213-219. https://doi.org/10.1007/s10493-013-9686-6

Oetcke E (1912) Histologische Beiträge zur Kenntnis der Verdauungs-vorgänge bei den Araneiden. Zool Jahrb Abth Allg Zool 31:245-276

O'Hagan JE (1974) Boophilus microplus: digestion of hemoglobins by the engorged female tick. Exp Parasitol 35(1):110-118

Ohara S, Homma T (1959) Morphology of Yato-byo ticks; internal structure of Haemaphysalis flava Neumann 1897, female. Jpn J Sanit Zool 10:104

Oleaga A, Obolo-Mvoulouga P, Manzano-Román R, Pérez-Sánchez R (2017) A proteomic insight into the midgut proteome of Ornithodoros moubata females reveals novel information on blood digestion in argasid ticks. Parasites Vectors 10:366-387. https://doi.org/10.1186/s13071-017-2300-8

Osaka H (1973) Electron microscopic observations of Chlamydia-like microorganisms in hepatopancreas cells of the spider Coras luctuosus. Acta Arachnol 25(1):23-29

Osterhoff DR, Gothe R (1966) The use of red cell antigens for timing break-down of erythrocytes during digestion in Ornithodoros savignyi (Audouin, 1827). Parasitology 56(4):613-618. https://doi. org/10.1017/S0031182000071626

Pagenstecher HA (1860) Beiträge zur Anatomie der Milben. Heft I.Trombidium holosericeum, Trombidium tinctorium. Leipzig, Verlag von Wilhelm Engelman

Pagenstecher HA (1861) Beiträge zur Anatomie der Milben. Heft II. Ixodes ricinus. Leipzig, Verlag von Wilhelm Engelman, pp 1-45 (2 plates)

Pantin CFA (1948) Notes on microscopical technique for zoologists. Cambridge University Press, Cambridge

Paran TP (1979) Evolution of the excretory duct in Myobia muris-musculi (Schrank, 1781) Myobiidae (Trombidiformes-Acarina). Acarologia 21(1):55-60

Paul KI, Polglase PJ, Smethurst PJ, OConnell AM, Carlyle CJ, Khanna PK (2004) Soil temperature under forests: a simple model for predicting soil temperature under a range of forest types. Agric For Meteorol 121:167-182

Pavlovsky EN, Zarin EJ (1926) On the structure and ferments of the digestive organs of scorpions. Q J Microsc Sci (n.s) 70(2):221-262

Pawlowsky EN (1927) Gifttiere und ihre Giftigkeit. Gustav Fischer Verlag, Jena, p 197

Pearse AGE (1960) Histochemistry, theoretical and applied, 2nd edn. Churchill

Perron R (1954) Untersuchungen über Bau, Entwicklung und Physiologie der Milbe Histiostoma laboratorium Hughes. Acta Zoologica Stockh 35(1-2):71-176

Peters W (1968) Vorkommen, Zusammensetzung und Feinstruktur peritorphischer Membranen im Tierreich Zeitschrift Morphol Ökol. Tiere 62:9-57

Petrova VI (1970) Reproductive system of the predatory mite Phytoseiulus persimilis. In: Papers from 2nd acarologic conference pt.2 Naukova Dumka, Kiev, pp 68-70 (in Russian)

Phillipson J (1961) Histological changes in the gut of Mitopus morio during protein digestion. Q J Microsc Sci 102:217-226 
Pickford GE (1942) Studies on the digestive enzymes of spiders. Trans Conn Acad Arts Sci 35:33-69

Piekarski G (1935) Beiträge zur Intracellulären Symbiose, Entwicklungsgeschichte und Anatomie Blutsaugender Gamasiden. Doctoral Thesis. Friedrich-Wilhelms-Universitat zu Berlin. Zeitschrift für Parasitenkunde 7:615-634

Plateau F (1876) Notes sur les phénomènes de digestion chez les Phalangides. Bull Acad Belg 42:719-754 (1 plate)

Plateau F (1877) Recherches de la structure de l'appareil digestif et sur le phénomène de la digestion chez les Aranéides dipneumones. Bull Acad R Sci Belge Bruxelles Ser 2 44(8):129-531

Pocock RI (1902) On some points in the anatomy of the alimentary and nervous systems of the arachnid suborder Pedipali. Proc Zool Soc Lond 2:169-188

Pollak PM (1966) Esterase activity in the Arachnida. Nat Lond 211:546-547

Pollock HM (1898) The anatomy of Hydrachna inermis Piersig. Inaugural-Dissertation der Hohen Philosophischen Fakultät der Universität Leipzig zurt Erlangung der Doctorwürde

Ponton TG (1868) The alimentary system of a house spider. Sci Gossip 128-131

Popow PP, Golzowa RD (1933) Zur Kenntnis der Wasserstoffionenkonzentration im Darmkanale einiger blutsaugender Arthropoden. Archiv fur Schiffs- und Tropenhygiene 37(10):465-466

Potgieter FT, Els HJ, van Vuuren AS (1976) The fine structure of merozites of Babesia bovis in the gut epithelium of Boophilus microplus. Onderstepoort J Vet Res 43(1):1-10

Prasse J (1967) Zur Anatomie und Histologie der Acaridae mit besonderer Berücksichtigung von Caloglyphus berlesei (Michael 1903) und C. michaeli (Oudemans 1924). I. Das Darmsystem. Wiss Zeit MartLuther-Univ Halle, math -nat R 16(5):789-812

Prasse J (1968a) Zur Anatomie und Histologie der Acaridae mit besonderer Berücksichtigung von Caloglyphus berlesei (Michael 1903) und C. michaeli (Oudemans 1924). II. Das Exkretionssystem und das Bindegewebe. Wiss Zeit Mart-Luther-Univ Halle math -nat R 16:963-970

Prasse J (1968b) Zur Anatomie und Histologie der Acaridae mit besonderer Berücksichtigung von Caloglyphus berlesei (Michael 1903) und C. michaeli (Oudemans 1924). III. Die Drüsen. Wiss Zeit MartLuther-Univ Halle math -nat R 17:629-646

Pritchard J, Kuster T, Sparagano O, Tomley F (2015) Understanding the biology and control of the poultry red mite Dermanyssus gallinae: a review. Avian Pathol 44(3):143-153

Proctor JR, Kendrick WB (1963) Unequal weighting in numerical taxonomy. Nature 197(4864):716-717

Prosi F, Storch V, Janssen HH (1983) Small cells in the mid-gut glands of terrestrial Isopoda: sites of heavy metal accumulation. Zoomorphology 102:53-64

Purrini K, Weiser J (1981) Eight new microsporidian parasites of moss mites (Oribatei, Acarina) in forest soils. Sonderdruck aus Bd 91(3):217-224

Raikhel AS (1974a) Revealing polysaccharides in ultra thin sections in the intestine and Malpighian tubules of the tick Hyalomma asiaticum. Tsitologiya 16(11):1418-1420 (in Russian)

Raikhel AS (1974b) An electron microscopic study of endocytosis in the mid-gut cells of an ixodid tick. Tsitologiya 16(12):1499-1504 (in Russian)

Raikhel AS (1975) Electron microscopic study of intracellular digestion in intestinal cells of the ixodid tick Hyalomma asiaticum. I. Formation of primary and secondary lysosomes. Tsitologiya 17(7):748-753 (in Russian)

Raikhel AS (1978) Electron microscope study of intracellular digestion in mid-gut cells of the tick Hyalomma asiaticum. II. Formation of residual bodies. Tsitologiya 20(2):142-148 (in Russian)

Raju DC, Bashamohidan MD, Narasimham C (1973) Circadian rhythm in blood glucose and liver glycogen levels of the scorpion. Heterometrus fulvipes. Experientia 29(8):964-965

Razumova IV (1977) Physiological age of imago ixodid ticks and a rapid method for its determination. Med Parazitol Parazit Bolezni 46(5):557-566 (in Russian)

Reggiori F (2006) Membrane origin for autophagy. Curr Top Dev Biol 74:1-30

Reich CI, Zorzópulos J (1978) Boophilus microplus: characterization of larval proteases. Exp Parasitol 44(1):1-6. https://doi.org/10.1016/0014-4894(78)90074-7

Reichenow E (1918) Digestion intracellular en un Ácaro. Bolletin Real Sociedad espanola Historia natural 18:258-273 (1 plate)

Reichenow E (1921) Die haemococcidien der Eidechsen. Vorbemerkungen und I. Teil: Die Entwicklungsgeschichte von Karyolysus. Archiv für Protistenkunde 42(2):179-291

Reichenow E (1922) Intracelluläre Symbionten bei blutsuagenden Milben und Egeln. Arch Protistenk 45:95-116

Reichle DE, Crossley DA Jr (1965) Radiocesium dispersion in a cryptozoan food web. Health Phys 11:1375-1384

Reid GDF, Boid R (1984) Assessment of temporal changes in heme and protein levels in ixodid ticks following blood engorgement, and their use as age-grading parameters. J Insect Physiol 30(12):975-978 
Reiff M (1949) Physiologische Merkmale bei Spinnmilben (Acari, Tetranychidae) nach Veränderung des Blattstoffwechsels. Verh Schweiz naturf Ges 129:165-166

Repkina LV (1976) Unevenness of the senescence of the intestinal epithelium of hungry ticks Ixodes persulcatus. Parazitologiya, Leningrad 10(6):526-530 (in Russian - also see Bull Analyt d'Entom Médicale et Vétérinaire (1977) 24(2):21)

Reuter E (1909) Zur Morphologie und Ontogenie der Acariden mit besonderer Berücksichtigung von Pediculopsis graminum (E.Reut.). Acta Societatis scientiarum Fennicae, Helsingfors 36(4):1-288

Richards AG, Brooks MA (1958) Internal symbiosis in insects. Annu Rev Entomol 3:37-56

Richards PR, Fry WG (1978) Digestion in pycnogonids: a study of some polar forms. Zool J Linn Soc 63(1-2):75-97. https://doi.org/10.1111/j.1096-3642.1978.tb02091.x

Ries E (1931) Die Symbiose der Läuse under Federlinge. Z Morphol Ökol Tiere 20:233

Robinson LE, Davidson J (1913-1914) The anatomy of Argas persicus (Oken, 1818). I-III. Parasitolology 6(1):20-48 (6(3):217-256; 6(4):382-424)

Rodriguez JG, Hampton RE (1966) Essential amino acids determined in the two-spotted spider mite, Tetranychus urticae Koch (Acarina, Tetranychidae) with glucose-U-C ${ }^{14}$. J Insect Physiol 12(10):12091210. https://doi.org/10.1016/0022-1910(66)90012-6

Roesler R (1934) Histologische, physiologische und serologische Untersuchungen über die Verdauung bei der Zeckengattung Ixodes Latr. Zeitschrift für Morphologie und Ökologie der Tiere 28(3):297-317

Rohde CJJr, Oemick DA, (1967) Anatomy of the digestive and reproductive systems in a acarid mite (Sarcoptiformes). Acarologia 9(3):608-616

Romijn C (1946) Verbleibszeit und Durchgangszeit der Nahrung bei Vertebraten und Invertebraten. Tabulae Biologicae, Hague 21:186-197

Roodyn DB (1967) Enzyme cytology. Academic Press, London, p 587

Rosell-Davis R, Coons LB (1989) Relationship between feeding, mating, vitellogenin production and vitellogenesis in the tick Dermacentor variablis. Exp Appl Acarol 7(1):95-105

Roshdy MA (1961a) Comparative internal morphology of subgenera of Argas ticks (Ixodoidea. Argasidae). 1. Carios: Argas vespertilionis Latreille, 1802. J Parasitol 47(6):987-994

Roshdy MA (1961b) Observations by electron microscopy and other methods on the intracelleular rickettsia-like microorganisms in Argas persicus Oken (Ixodoidea, Argasidae). J Insect Pathol 3:148-166

Roshdy MA (1962) Comparative internal morphology of subgenera of Argas ticks (Ixodoidea. Argasidae). 2. Subgenus Chiropterargas: Argas boueti Robaud and Colas-Belcour, 1933. J Parasitol 48(4):623-630

Roshdy MA (1963) Comparative internal morphology of subgenera of Argas ticks (Ixodoidea. Argasidae). 3. Subgenus Secretargas: Argas transgariepinus White, 1846. J Parasitol 49(5):851-856

Roshdy MA (1966) Comparative internal morphology of subgenera of Argas ticks (Ixodoidea. Argasidae). 4. Subgenus Ogadenus: Argas brumpti Neumann, 1907. J Parasitol 52(4):776-782

Roshdy MA, Marzouk AS (1984) The subgenus Persicargas (Ixodoidea: Argasidae: Argas): Argas arboreus central nervous system anatomy and histology. J Parasitol 70(5):774-787

Rothstein A, Meier R, Scharff T (1953) Relationship of cell surface to metabolism IX. Digestion of phosphorylated compounds by enzymes located on surface of intestinal cell. Am J Physiol 173:41-46

Rudin W, Hecker H (1979) Functional morphology of the mid-gut of Aedes aegypti L. (Insecta, Diptera) during blood digestion. Cell Tissue Res 200(2):193-203

Rudsinska MA, Spielman A, Lwengrub S, Piesman J, Karakashian S (1982) Penetration of the peritrophic membrane of the tick by Babesia microti. Cell Tissue Res 221(3):471-481

Sacchi L, Genchi M, Clementi E, Bigliardi E, Avanzati AM, Pajoro M, Negri I, Marzorati M, Gonella E, Alma A, Daffonchio D, Bandi C (2008) Multiple symbiosis in the leafhopper Scaphoideus titanus (Hemiptera: Cicadellidae): details of transovarial transmission of Cardinium sp. and yeast-like endosymbionts. Tissue Cell 40(4):231-242

Sabelis MW (1990) How to analyse prey preference when prey density varies? A new method to discriminate between effects of gut fullness and prey type composition. Oecologia 82:289-298

Saito Y (1960) Studies on ixodid ticks. Part 4. The internal anatomy in each stage of Haemaphysalis flava Neumann, 1897. Acta Medica etbiologica Nigata 8(3):189-239

Samson K (1909) Zur Anatomie und Biologie von Ixodes ricinus L. Zeitschrift für wiss Zool 93:185-236

Sandness JN, McMurtry JA (1972) Prey consumption behavior of Amblyseius largoensis in relation to hunger. Can Entomol 104(4):461-470. https://doi.org/10.4039/Ent104461-4

Santamaría ME, Arnaiz A, Diaz-Mendoza M, Martinez M, Díaz I (2015a) Inhibitory properties of cysteine protease pro-peptides from barley confer resistance to spider mite feeding. PLoS One 10(e0128323):1-17. https://doi.org/10.1371/journal.pone.0128323

Santamaría ME, González-Cabrera J, Martínez M, Grbić V, Castañera P, Díaz I, Ortego F (2015b) Digestive proteases in bodies and faeces of the two-spotted spider mite, Tetranychus urticae. J Insect Physiol 78:69-77. https://doi.org/10.1016/j.jinsphys.2015.05.002 
Schaub R von (1888) Über der Anatomie von Hydrodroma (C.L.Koch). Ein Beitrag zur Kenntnis der Hydrachniden. Sitz Ber K Akad Wiss Wien 97(1):98-151

Schlottke E (1933) Darm und Verdauung bei Pantopoden. Zeitschrift mikro-anat Forsch 32:633-658

Schlottke E (1934a) Unterschiede in der Entwicklung des phagozytierenden und des resorbierenden Darmepithels. Biol Zentralbl 54(1-2):51-64

Schlottke E (1934b) Histologische Beobachtungen über die intrazelluläre Verdauung bei Dendrocoelum lacteum (Müll.) und Euscorpius carpathicus (L.). Sitzungsberichte und Abhandl der Naturforschenden Gesellschaft zu Rostock Ser 3 4:76-86

Schlottke E (1936) Über die Verdauungsfermente der Vogelspinnen. Sitzungsberichte und Abhandl der Naturforschenden Gesellschaft zu Rostock ser 3(6):89-106

Schmidt U (1935) Beiträge zur Anatomie und Histologie der Hydracarinen, besonders von Diplodontus despiciens O.F.Muller. Z für Morph u Ökol Tiere 30(2):99-176

Schnieder-Berkenbosch L (1955) Some remarks about the digestive tract and the female reproductive system of the fruit tree red spider mite, Metatetranychus ulmi (Koch). Proc Akad Wet Amst 58C:489-494

Schöneich D (1970) Hemmwirkung von Phosphorsäureestern und Carbamatenauf die Esterasen verschiedener Stämme von Spinnmilben der Gattung Tetranychus. Zeit F Angew Zool 57:97-119

Schramlová J (1978a) Microscopical anatomy of larva of Cheladonta costulata (Acarina: Trombiculidae). I. Glands. Folia Parasitologica (Praha) 25(1):61-65

Schramlová J (1978b) Microscopical anatomy of larva of Cheladonta costulata (Acarina: Trombiculidae). II. Body tegument and digestive system. Folia Parasitologica (Praha) 25(2):179-182

Schulze P (1943) Die Gestaltung des Mitteldarmes bei den Zecken und die Einrichtungen für die Körperdehnung bei der Blutaufname (nebst Beiträgen zur Lebensgeschichte der Ixodoidea). Zeitschrift für Morphologie und Ökologie der Tiere 39(2):320-368

Schütte C, Dicke M (2008) Verified and potential pathogens of predatory mites (Acari: Phytoseiidae). Exp Appl Acarol 46(1-4):307-328

Schweizer J (1961) Die Landmilben der Schweiz. Parasitiformes. Denkschriften der Schweizerischen Naturforschenden Gesellschaft 84:1-207

Seitz K-A (1972) Elektronenmikroskopische Untersuchungen an den Guanin-Speicherzellen von Araneus diadematus Clerk (Araneae, Araneidae). Zeitschrift für Morphologie Tiere 72(3):245-262

Serfaty A (1941a) Pouvoir catalasique des organes du scorpion. C R Soc Biol Paris 135:7-8

Serfaty A (1941b) Variation de l'activité catalasique, au cours de la digestion, chez les Scorpions adultes. C R Soc Biol Paris 135(9-10):653-654

Serfaty A (1942a) Variation des acides aminés libres et de la tryptase au cours de la digestion chez les Scorpions. Bulletin de la Société entomologique de France 47(3-4):42-45

Serfaty A (1942b) Influence du jeûne, précédant le repas, sur l'activité trypsique, au cours de la digestion, chez les Scorpions. Bulletin de la Société entomologique de France 47(5-6):89-90

Shatrov AB (1983) Morphological and functional specialization of tubular glands in trombiculid mite larvae (Trombiculidae). Parazitologiya 17(2):134-139

Shatrov AB (2010) Comparative morphology, ultrastructure and functions of the excretory organ (postventricular mid-gut) in the Parasitengona (Acariformes). Acarologia 50(1):93-112

Sidorov VE (1960a) Gut of argasid ticks as inhabitation environment of Borrelia sogdianum. Zool Zh 39(9):1324-1329 (in Russian)

Sidorov VE (1960b) The body cavity of argasid ticks as a habitat of spirochetes and Brucella. Zh Mikrobiol Moskva 31(6): 1-7

Sig Thor (1930) Beiträge zur Kenntnis der invertebraten Fauna von Svalbard. Skrifter om Svalbard og Ishavet 27:1-156

Silvere AP (1971) Origin and evolution of gall mites (Eriophyoidea) in the light of their internal anatomy. In: Proceedings 3rd international congress of acarology, Prague, pp 247-249

Sinha RC, Kanungo MS (1967) Effect of starvation on the scorpion Palamnaeus bengalensis. Physiol Zool 40:386-390

Sixl W (1973) Morphologisch-histologische Untersuchungen bei Ascoschöngastia latyshevi Schluger (Trombiculidae, Acari). Zool Anz 191(3-4):235-257

Smit JDG, Grandjean O, Guggenheim RR, Winterhalter KH (1977) Haemoglobin crystals in the midgut of the tick Ornithodorus moubata Murray. Nat Lond 266(5602):536-538

Smrž J (1989) Internal anatomy of Hypochthonius rufulus (Acari: Oribatida). J Morphol 200:215-230

Smrž J (2002) Microanatomical and microbiological characteristics of the quiescent state of Scutovertex minutus (Acari: Oribatida). Exp Appl Acarol 27:103-112

Smrž J (2003) Microanatomical and biological aspects of bacterial associations in Tyrophagus putrescentiae (Acari: Acaridida). Exp Appl Acarol 31(1-2):105-113 
Smrž J, Čatská (1989) Food selection of the field population of Tyrophagus putrescentiae (Schrank) (Acari: Acaridida). J Appl Entomol 104:1227-1232

Sneath PHA, Sokal RR (1973) Numerical taxonomy: the principles and practice of numerical classification, 2nd edn. W H Freeman, San Francisco, p 359

Snodgrass RE (1948) The feeding organs of Arachnida including mites and ticks. Smithsonian Misc Coll 110(10):1-93

Snodgrass RE (1952) A text-book of arthropod anatomy. Comstock, New York

Snow KR (1970) The quantity of blood imbibed by Hyalomma anatolicum anatolicum Koch, 1844 (Ixodoidea, Ixodidae). Parasitology 60(1):53-60

Sobotnik J, Alberti G, Weyda F, Hubert J (2008a) Ultrastructure of the digestive tract in Acarus siro (Acari: Acaridida). J Morphol 269(1):54-71

Sobotnik J, Kudlikova-Krizkova I, Vancova M, Munzbergova Z, Hubert J (2008b) Chitin in the peritrophic membrane of Acarus siro (Acari: Acaridae) as a target for novel acaricides. J Econ Entomol 101(3):1028-1033

Sojka D, Franta Z, Horn M, Hadjusek O, Caffrey CR, Mareš M, Kopáček P (2008) Profiling of proteolytic enzymes in the gut of the tick Ixodes ricinus reveals an evolutionary conserved network of aspartic and cysteine peptidases. Parasites Vectors 1(1):7

Sojka D, Franta Z, Horn M, Caffrey CR, Mareš M, Kopáček P (2013) New insights into the machinery of blood digestion by ticks. Trends Parasitol 29(6):276-285

Sojka D, Pytelkova J, Perner J, Horn M, Konvičková J, Schrenková J, Mareš M, Kopáček P (2016) Multienzyme degradation of host serum albumin in ticks. Ticks Tick Borne Dis 7(4):604-613

Sonenshine DE (1970) A contribution to the internal anatomy and histology of the bat tick, Ornithodoros kelleyi. 2. The reproductive, muscular, respiratory, excretory and nervous systems. J Med ENT 7(3):289-312

Sonenshine DE, Gregson JD (1970) A contribution to the internalanatomy and histology of the bat tick Ornithodoros kelleyi Cooleyand Kohls, 1941. I. The alimentary system, with notes on the foodchannel in Ornithodoros denmarki Kohls, Sonenshine, and Clifford,1965. J Med Entomol Honolulu 7(1):46-64

Sonenshine DE, Roe RM (1991) Biology of ticks: volume 1. Oxford University Press, Oxford

Sorensen JT, Kinn DN, Doutt RL, Cate JR (1976) Biology of the Mite, Anystis agilis (Acari: Anystidae): a California vineyard predator. Ann Entomol Soc Am 69(5):905-910. https://doi.org/10.1093/ aesa/69.5.905

Stammer H-J (1963) Beiträge zur Systematik und ökologie Mitteleuropäischer Acarina Band II (1) Akademische Verlagsgesellschaft, Leipzig

Stanley J (1931) Studies on the musculatory system and mouth parts of Laelaps echidninus Berl. Ann Entomol Soc Am 24(1):1-18. https://doi.org/10.1093/aesa/24.1.1

Stanyukovich AK (1966) Preliminary data on the study of free amino-acids in ixodid and argasid ticks. Tezisy Dokl 1 Akarol Soveshich (in Russian)

Starovir IS (1973) Some peculiarities in the structure of the digestive and excretory systems in Phytoseiulus persimilis Athias Henriot (Parasitiformes. Phytoseiidae). Vestnik Zoologii 7(5):72-77 (in Russian)

Starovir IS (1979a) Characteristics of the structure of the digestive system in the mite Amblyseius herbarius (Gamasoidea. Phytoseiidae). Vestnik Zoologii 0(2):24-27 (in Russian)

Starovir IS (1979b) Functional histology in the intestinal epithelium in Amblyseius herbarius (Gamasoidea. Phytoseiidae). Vestnik Zoologii 0(3):40-44 (in Russian)

Starovir IS (1979c) Morphology of the digestive system of mites Amblyseius umbraticus (Gamasoidea, Phytoseiidae). Vestnik Zoologii 0(6):84-85 (in Russian)

Starovir IS (1980) Histology of digestive system of Androlaelaps casalis (Gamasoidea, Parasitiformes). Vestnik Zoologii 0(3):13-17

Starovir IS (1981a) Functional histology of the digestive system of the facultative blood-sucking fly Androlaelaps casalis (Parasitiformes, Gamasoidea). Vestnik Zoologii 0(2):47-52 (in Russian)

Starovir IS (1981b) Functional intestinal histology of adult mites Amblyseius umbraticus Parasitiformes Phytoseiidae. Vestnik Zoologii 0(5):62-66 (in Russian)

Starovir IS (1982) Functional digestion histology in Amblyseius reductus (Parasitiformes, Phytoseiidae). Vestnik Zoologii 0(1):69-74

Starovir IS (1985) Functional histology of digestive processes in the larvae, protonymphs and protochrysalids of the Mite Varroa jacobsoni (Parasitiformes, Varroidae). Vestnik Zoologii 0(6):51-87

Starovir IS, Barabanova VV (1981) Food digestion in the mites Phytoseiulus persimilis, Amblyseius andersoni and Amblyseius reductus (Gamasoidea, Phytoseiidae). Vestnik Zoologii 0(1):77-79 (in Russian)

Steding E (1924) Zur Anatomie und Histologie von Halarachne otariae n. sp. Zeitscrift wiss Zool 121(3):442-493 
Stella E (1942) Anatomia e istologia di Rhipicephalus sanguineous. Arch Zool (ital) Torino 30:155-180

Stepanchenok-Rudnik GI, Garmaev ATs, Ghrokhovskaya IM, (1975) Free amino-acids in the organs of Alveonasus lahorensis ticks. Med Parazitol Parazit Bolezn 44(5):549-553 (in Russian)

Stepanchenok-Rudnik GI, Potashova LA, Grokhovskaia IM (1971) Nitrogen and phosphorus content in the intact organism and in some organs of blood-sucking ticks (Ixodoidea). Med Parazitol (Mosk) 40(5):584-7 (in Russian)

Stout VM (1953) Eylais waikawae n.sp. (Hydracarina) and some features of its life history and anatomy. Trans R Soc N Zeal 81:389-416

Stradal-Schuster H (1940-1941) Aussenverdauung bei Spinnen. Verh Zool -Bot Ges Wien 90-91:83-128

Stromberg B, Nutting W (1979) Adaptive features of the exoskeleton and "pigment" deposits in Demodex spp. (Demodicidae). Acarologia 14:605 (1972)

Sudd JH (1952) Laboratory studies of adult female Bdellonyssus bacoti (Hirst, 1916) (Acarina, Parasitiformes). Ann Trop Med Parasitol 46(2):158-64

Strandtmann RW, Wharton GW (1958) A manual of mesostigmatid mites parasitic on vertebrates. Contribution No. 4 of the Institute of Acarology, Department of Zoology, University of Maryland

Summers FM, Gonzalez RRH, Witt RL (1973) The mouthparts of Bryobia rubrioculus (Sch.) (Acarina: Tetranychidae). Proc Entomol Soc Wash 75(1):96-111

Šustr V, Hubert J (1999) The influence of thermal acclimation on the amylolytic activity and microanatomy of the alimentary tract of the oribatid mite Galumna elimata (Acari: Oribatei). Eur J Entomol 96:189-198

Šustr V, Starý J (1998) Digestive enzymes in oribatid mites: Impact factors In: (eds) Pižl V, Tajovský K, Soil zoological problems in Central Europe, Publisher: ISB AS CR České Budějovice, pp 23-24

Sutton E, Arthur DR (1962) Tick feeding in relation to disease transmission. Zool Soc Lond Symp 6:223-252

Suvorov EK (1908) Ixodes reduvius. An anatomical study. Trudy Imp S. -Peterb Obsc Estetr, Vypusk 4 Otd Zool Fiziol (St Peterburg, Trav laborat zool) 38(19):137-224 ((in Russian) + German summary p225-240)

Talarico G, Lipke E, Alberti G (2011) Gross morphology, histology, and ultrastructure of Ricinulei (Arachnida) with emphasis on functional and phylogenetic implications. J Morphol 272(1):89-117. https:// doi.org/10.1002/jmor.10897

Tarman K (1968) Anatomy, histology of oribatid gut and their digestion. Biol Věstn 16:67-76

Tarnowski BI, Coons LB (1989) Ultrastructure of the mid-gut and blood meal digestion in the adult tick Dermacentor variabilis. Exp Appl Acarol 6(4):263-289

Tarras-Wahlberg N (1960) Taxonomical, anatomical and biological comments on Diapterobates humeralis (Hermann, 1804)_Oribatei, Acari-including a description of the tritonymph. Kungl Fisiografiska Sällskapets I Lund Förhandlingar 30(12):91-101

Tatchell RJ (1962) The digestion of blood proteins by the tick, Argas persicus. Parasitology 52:13P-14P

Tatchell RJ (1964) Digestion in the tick, Argas persicus Oken. Parasitology 54(3):423-440

Tatchell RJ, Araman SF, Boctor FN (1972) Biochemical and physiological studies of certain ticks (Ixodoidea). Protease activity cycles in Argas (Persicargas) persicus (Oken) and A. (P.) arboreus Kaiser, Hoogstraal, and Kohls (Argasidae). Zeitschr Parasitenkd 39(4):345-350

Tatchell RJ, Kerr JD, Boctor FN (1973) Biochemical and physiological studies of certain ticks (Ixodidae). Haemolysis rate and meal size in the interactions between Argas (Persicargas) arboreus Kaiser, Hoogstraal and Kohls, Argas (Persicargas) persicus (Oken) (Argasidae) on some avian hosts. Parasitology $67: 41-51$

Teravsky IK (1951) Influence of the temperature on the rate of blood digestion in Ornithodorus papillipes. Zool Zh 30:440-444 (in Russian)

Teravsky IK (1957) On the formed elements of the haemolymph of ticks of the family Argasidae. Zool Zh 36(10):1448-1454 (in Russian)

Thomae H (1925) Beiträge zur Anatomie der Halacariden. Zool Jb Anat 47(1):155-190

Thon K (1903) Recherches sur l'anatomie comparée des Acariens Prostigmatiques. Ann Sci Nat Zool 19:1-228

Thon K (1905a) Neue Exkretionsorgane bei der Hydrachnidenfamilie Limnocharidae Kramer. Zeitschr wiss Zool 97(4):465-495

Thon K (1905b) Monographie der Holothyriden. Sitz B naturw Ges, Prag, p 4

Thon K (1905c) Über die Coxaldrüsen bei Holothyriden. Zool Anz 28:823-832

Thon K (1905d) Über die Drüsen der Holothyriden. Sitzungsberichte der K. Böhmischen Gesellschaft der Wissenschaften, Prague, pp 1-41 (2 plates)

Thor S (1902) Eigenartige, bisher unbekannte Dr/"usen bei einzelnen "Hydrachniden" - Formen. Zoologische Anzeiger 25(672):401-409 
Thor Sig TST (1904) Récherches sur l'anatomie comparée des Acariens prostigmatiques. Annales des Sciences Naturelles (Zoologie) Ser 8 19:1-190

Thor Sig (1931) Einführung in das Studium der Acarina (Milben). Die Tierwelt Deutschlands und der angrenzenden Meeresteile nach ihren Merkmalen und nach ihrer Lebensweise. (ed) Dahl F, Teil 22. Spinnentiere oder Arachnoidea. V: Acarina. Verlag von Gustav Fischer, Jena, pp 1-78

Till WM (1961) A contribution to the anatomy and histology of the brown ear tick Rhipicephalus appendiculatus Neumann. Mem Entomol Soc S Afr 6:1-124 (21 plates)

Tongu Y, Ishij A, Oh H (1986) Ultrastructure of house dust mites, Dermatophagoides farinae and Dermatophagoides pteronyssinus. Jpn J Sanit Zool 37:237-244

Trager W (1939) Intracellular microörganism-like bodies in the tick Dermacentor variabilis Say. J Parasitol 25(3):233-238

Treat AE (1975) Mites of moths and butterflies. Comstock. Cornell University Press, London, p 362

Treviranus GR (1812) Ueber den innern Bau der Arachniden. Herausgegeben von der physikalisch-medicinischen Societät in Erlangen. Johann Leonhard Schrag, Nürnberg. 1:1-48 (5 plates)

True GH (1932) Studies of the anatomy of the Pajaroello tick, Ornithodoros coriaceus Koch. I. The alimentary canal. Univ Calif Publ Entomol 6(3):21-48

Tsvileneva VA (1959) Formed elements of the hemolymph of ixodid ticks. Dokl Stalinabad Akad Nauk Tadzhikist SSR 2(1):45-51 (in Russian)

Tsvileneva VA (1961a) Loose connective tissue in ixodid ticks. Arkh Anat Gistol Embriol 41(12):79-88 (in Russian)

Tsvileneva VA (1961b) On the adipose body in ixodid ticks. Dokl Akad Nauk Tadzhikist SSR 4(2):57-60 (in Russian)

Tsvileneva VA (1963) On the morphology of the connective tissue of ixodid ticks. Dokl Akad Nauk Tadzhikist SSR 24:171-177 (in Russian)

Turk FA, Phillips SM (1946) A monograph of the slug mite Riccardoella limacum (Schrank). Proc Zool Soc Lond 115:448-472

Ugolev AM (1965) Membrane (contact) digestion. Physiol Rev 45:555-595

van der Hammen L (1977) A new classification of Chelicerata. Zoologische Mededelingen 51(20):307-319 van der Hammen L (1989) An introduction to comparative arachnology. SPB Academic Publishing, Leiden van der Borght O (1966) Peritrophic membranes in Arachnida (Arthropoda). Nature (Lond) 210:751-752

Vasquez AA (2017) The digestive composition and physiology of water mites. Wayne State University Dissertations, Detroit Michigan. https://digitalcommons.wayne.edu/oa_dissertations/1887

Vidal-quist JC, Ortego F, Rombauts S, Castañera P, Hernández-crespo P (2017) Dietary shifts have consequences for the repertoire of allergens produced by the European house dust mite. Med Vet Entomol 31:272-280. https://doi.org/10.1111/mve.12234

Vidomsky EV, Balykin AV, Romasheva LF, Shcherbak VP (1973) Histopathological structure characteristics of internal organs and hemolymph of the tick Argas persicus under the effect of Bacillus thuringiensis cultures. Izv Akad Nauk Kirgiz SSR 0(6):79

Vijayambika V, John PA (1974) Internal morphology and histology of the fish mite Lardoglyphus konoi (Sasa and Asanuma) Acarina: Acaridae 1. Digestive system. Acarologia 16:709-718

Vijayambika V, John PA (1975a) Internal morphology and histology of the fish mite Lardoglyphus konoi (Sasa and Asanuma) (Acarina: Acaridiae). 1 Digestive system. Acarologia 16(4):709-718

Vijayambika V, John PA (1975b) Internal morphology and histology of the fish mite Lardoglyphus konoi (Sasa and Asanuma) (Acarina: Acaridiae). 2. Reproductive system. Acarologia 17(1):106-113

Vijayambika V, John PA (1975c) Internal morphology and histology of the fish mite Lardoglyphus konoi (Sasa and Asanuma) (Acarina: Acaridiae). 3. Nervous system. Acarologia 17(1):114-119

Vijayambika V, John PA (1975d) Functional morphology of the alimentary canal of the fish mite Lardoglyphus konoi. Forma Functio 8(3-4):387-394

Vijayambika V, John PA (1976a) Internal morphology and histology of the post-embryonic stages of the fish mite Lardoglyphus konoi (Sasa and Asanuma) Acarina: Acaridae 1, Larva. Acarologia 17(4):745-750

Vijayambika V, John PA (1976b) Internal morphology and histology of the post-embryonic stages of the fish mite Lardoglyphus konoi (Sasa and Asanuma) Acarina: Acaridae 2, Protonymph. Acarologia 18(1):133-137

Vijayambika V, John PA (1976c) Internal morphology and histology of the post-embryonic stages of the fish mite Lardoglyphus konoi (Sasa and Asanuma) Acarina: Acaridae 3, Deutonymph. Acarologia 18(1):138-142

Vijayambika V, John PA (1977) Digestive system of Aleuroglyphus ovatus (Acarina: Tyroglyphidae). Indian J Acarol 2(1):24-29

Vinogradova GA (1960) Data on the anatomy and histology of certain gamasid mites. Nauch Trudy Kalinninsk Otdel Mosk O-va I spyt Prirody 0(2):63-73 (in Russian) 
Vistorin HE (1980) Ernährungsbiologie und Anatomie der Verdauungstraktes der Nicoletiellidae (Acari, Trombidiformes). Acarologia 21(2):204-215

Vistorin-Theis G (1977a) Ernährungsbiologische Beobachtungen an Calyptostomiden (Acari, Trombidiformes). Zool Anz Jena 199(5/6):381-385

Vistorin-Theis G (1977b) Anatomische Untersuchungen an Calyptostomiden (Acari, Trombidiformes). Acarologia 19(2):242-257

Vitzthum GH (1940) Acarina In: Dr H G Bronn's: Klassen und Ordnungen des Tierreiches, Akademische Verlagsgesellschaft Becker \& Erler Leipzig. Band 5, Abteilung IV, Buch 5, Lieferung 1-7, pp 1-1011 (Leipzig)

Volkonsky M (1940) Podapolipus diander n.sp. Acarien Hétérostygmate parasite du ctiquet migrateur (Locusta migratoria L.). Arch Institut Pasteur d'Alg/'erie 18(3):321-340

Vonk HJ (1962) Emulsifiers in the digestive fluids of invertebrates. Arch Int Physiol Biochim 70:67-85

Waladde SM, Kemp DH, Rice MJ (1979) Feeding electrograms and fluid uptake measurements of cattle tick Boophilus microplus attached on artificial membranes. Int J Parasitol 9(2):89-95. https://doi. org/10.1016/0020-7519(79)90096-1

Wales W (1976) Receptors of the mouth parts and guts of arthropods. In: Mill PJ (ed) Structure and function of proprioreceptors in the invertebrates. Chapman and Hall, London, pp 213-241

Walling MV, White DC, Rodriguez JG (1968) Characterization, distribution, catabolism, and synthesis of the fatty acids of the two-spotted spider mite, Tetranychus urticae. J Insect Physiol 14(10):14451458. https://doi.org/10.1016/0022-1910(68)90178-9

Wallwork JA (1970) Ecology of soil animals. McGraw-Hill, London, p 283

Wallwork JA (1983) Oribatids in forest ecosystems. Ann Rev Entomol 28:109-130

Walter A, Bechsgaard J, Scavenius C, Dyrlund TS, Sanggaard KW, Enghild JJ, Bilde T (2017) Characterisation of protein families in spider digestive fluids and their role in extra-oral digestion. BMC Genom 18:600-613. https://doi.org/10.1186/s12864-017-3987-9

Walter DE, Proctor HC (1998) Feeding behaviour and phylogeny: observations on early derivative Acari. Exp Appl Acarol 22(1):39-50

Walzl MG (1992) Ultrastructure of the reproductive system of the house dust mite Dermatophagoides farinae and Dermatophagoides pteronyssinus (Acari, Pyroglyphidae) with remarks on spermatogenesis and oogenesis. Exp Appl Acarol 16:85-116

Wang YM, Liu XY, Jiang CL, Huang LN, Sun X, Liu ZG (2013) Ultrastructure of the digestive system in Dermatophagoides farinae (Acariformes: Pyroglyphidae). Zhongguo Ji Sheng Chong Xue Yu Ji Sheng Chong Bing Za Zhi 31(6):489-492 (in Chinese)

Warburg MR (2012) Reviewing the structure and function of the scorpion's hepatopancreas. Arthropods 1(3):79-93

Warren E (1940) On the genital system of Dermanyssus gallinae, and several other Gamasidae. Ann Natal Mus 9:409-459

Warren E (1941) On the genital system and modes of reproduction and dispersal in certain gamasid mites. Ann Natal Mus 10:95-126

Warren E (1944) Observations on the anatomy and histology of a myrmecophilous mite (Urodinychus sp.) and an account of certain of its sporozoan parasites. Ann Natal Mus 10:359-406

Warren E (1947) On the genital system and gut of the oribatid mite, Cepheus tegeocranus, and the reaction of these organs to a ray-fungus parasite. Ann Natal Mus 11(1):1-36

Weber G (1974) Glycol methacrylate embedding in enzyme histochemistry: application to arthropode tissue incubated for demonstration of unspecific esterase and succinic dehydrogenase activity. Histochemistry 39(2): 155161

Weghofer M, Grote M, Resch Y, Casset A, Kneidinger M, Kopec J, Thomas WR, Fernández-Caldas E, Kabesch M, Ferrara R, Mari A, Purohit A, Pauli G, Horak F, Keller W, Valent P, Valenta R, Vrtala S (2013) Identification of Der p 23, a Peritrophin-like Protein, as a New Major Dermatophagoides pteronyssinus Allergen Associated with the Peritrophic Matrix of Mite Fecal Pellets. J Immunol 190(7):3059-3067

Weiser J (1972) The Entomophthoraceae (Phycomycetes) affecting tetranychid mites. In: Proceedings of the XIII international congress of entomology, Moscow 2-9 Aug 1968 Leningrad, pp 110-111

Weiser J, Daniel M (1969) Two mycotic infections in nidicolous mites. Folia Parasitol (Praha) 16(3):275-278

Weitz B, Buxton PA (1953) The rate of digestion of blood meals of various haematophagous arthropods as determined by the precipitin test. Bull Entomol Res 44(3):445-450. https://doi.org/10.1017/S0007 485300025566

Welsh U, Storch V (1976) Comparative animal cytology and histology. Sidgwick and Jackson, London 
Wernz JG, Krantz GW (1976) Studies on the function of the tritosternum in selected Gamasida (Acari). Can J Zool 54(2):202-213

Weygoldt P (1998) Review. Evolution and systematics of the Chelicerata. Exp Appl Acarol 22(2):63-79

Wharton GW, Fuller HS (1952) A manual of the chiggers. The biology, classification, distribution and importance to man of the larvae of the family Trombiculidae (Acarina). Mem Entomol Soc Wash $4: 1-175$

Wharton GW, Brody AR (1972) The peritrophic membrane of the mite, Dermatophagoides farinae: Acariformes. J Parasitol 58(4):801-804

Whitmoyer RE, Nault LR, Bradfute OE (1972) Fine structure of Aceria tulipae (Acarina: Eriophyidae). Ann Entomol Soc Am 65(1):201-215

Wiegert RG, Petersen CE (1983) Energy transfer in insects. Ann Rev Entomol 28:455-486

Wiesmann R (1968) Untersuchungen über die Verdauungsvorgänge bei der gemeinen Spinnmilbe Tetranychus urticae Koch. Zeitschrift für angew Ent 61:457-465

Williams SR (1905) Anatomy of Boophilus annulatus Say. Proc Boston Soc Nat Hist 32(8):1-334

Winkler W (1886) Das Herz der Acariden nebst vergleichenden Bemerkungen über das Herz der Phalangiiden und Chernetiden. Arbeit d Zool Inst zu Univ Wien 7(1):111-118 (1 plate)

Winkler W (1888) Anatomie der Gamasiden. Arbeiteten aus dem Zoologischen Institute der Universität Wien und der Zoologischen Station in Triest 7(3):317-354

Witalinski W (1975) Fine structure of vas deferens wall in the mite, Pergamasus viator Halas. (Mesostigmata, Parasitidae) (Acari). Acarologia 17(2):197-207

Witalinski W (1980) Fine structure of the respiratory system in mites from the family Parasitidae (Mesostigmata, Acari). Acarologia 21(3-4):331-339

With CJ (1904) The Notostigmata: a new sub-order of Acari. Vidensk ab Medd Dansk naturh hist Foren, Kjöbenhavn 56:137-192

Witte H (1972) Funktionsanatomische und ethnologische Untersuchungen an der Milbenfamilie Erythraeidae. Dissertation zur Erlangung des Doktorgrades der Mathematisch-Naturwissenschaftlichen Fakultät der Christian-Albrechts-Universität zu Kiel

Witte H, Storch V (1974) Licht- und elektronenmikroskopische Untersuchungen an Hodensekreten und Spermien der trombidiformen Milbe Abrolophus rubipes (Trouessart, 1888). Acarologia 15(3):441-450

Woodring JP (1973) Comparative morphology, functions, and homologies of the coxal glands in oribatid mites (Arachnida: Acari). J Morphol 139(4):407-429. https://doi.org/10.1002/jmor.1051390404

Woodring JP, Carter SC (1974) Internal and external morphology of the deutonymph of Caloglyphus boharti (Arachnida: Acari). J Morphol 144(3):275-296

Woodring JP, Cook EF (1962) The internal anatomy, reproductive physiology and molting process of Ceratozetes asalpinus. Ann Entomol Soc Am 55:164-181

Woodring JP, Galbraith CA (1976) The anatomy of the adult uropodid Fuscuropoda agitans (Arachnida: Acari) with comparative observations on other Acari. J Morphol 150(1):19-58

Wright KA, Newell IM (1964) Some observations on the fine structure of the mid-gut of the mite Anystis sp. Ann ENT Soc Am 57(6):684-693

Wright JD, Hastriter MW, Robinson DM (1984) Observations on the ultrastructure and distribution of Rickettsia tsutsugamushi in naturally infected Leptotrombidium arenicola (Acari: Trombiculidae). J Med ENT 21(1):17-27

Wu J, Yang F, Liu Z, Wu G, Ran P (2009) The alimentary canal of Blomia tropicalis (Acari: Astigmata: Echmopodidae): the application of three-dimensional reconstruction technology. Exp Appl Acarol 47(3):215-224

Wu K, Hoy MA (2012) Extended starvation reduced and eliminated Wolbachia, but not Cardinium, from Metaseiulus occidentalis females (Acari: Phytoseiidae): a need to reassess Wolbachia's status in this predatory mite? J Invertebr Pathol 109(1):20-26

Wyss-Huber von M, Striebel H, Wiss H, Geigy R (1961) Papierchromatographischer Nachweis verschiedener Zuker und spectrophotometrische Bestimmung des Glykogens in Extrakten von Glossinen, Reduviiden und Ornithodoros moubata. Acta Trop 18:46-57

Yonge CM (1928) Feeding mechanisms in the invertebrates. Biol Rev 3:21-76

Yonge CM (1937) Evolution and adaptation in the digestive system of the metazoa. Biol Rev 12:87-115

Yonge CM (1954a) Physiological anatomy of the alimentary canal in invertebrates. Tabulae Biologicae 21 3(20):1-24

Yonge CM (1954b) Feeding mechanisms in the Invertebrata. Tabulae Biologicae 21 Part 3(22):57-68

Young JH (1968a) The morphology of Haemogamasus ambulans. 1. Alimentary canal (Acarina: Haemogamasidae). J Kansas ENT Soc 41(1):101-107 
Young JH (1968b) The morphology of Haemogamasus ambulans. 2. Reproductive system. (Acarina: Haemogamasidae). J Kansas Ent Soc 41:532-543

Zamsky SI (1964) Nutrition of the gamasid mite Eulaelaps stabularis C. L. Koch and Haemolaelaps glasgowi Ew. (Laelaptidae) inhabiting some holes of mouse-like rodents. Ectoparasites Fauna Biol Pract Signif 4(4):259-283

Zhang YY, Sun X, Liu ZG (2008) Morphology and three-dimensional reconstruction of the digestive system of Dermatophagoides farinae. Int Arch Allergy Immunol 146(3):219-226

Zhu Z, Gern L, Aeschlimann (1991) The peritrophic membrane of Ixodes ricinus. Parasitol Res 77(7):635-641

Publisher's Note Springer Nature remains neutral with regard to jurisdictional claims in published maps and institutional affiliations.

\section{Affiliations}

\section{Clive E. Bowman ${ }^{1}$}

Clive E. Bowman

bowman@maths.ox.ac.uk

1 Mathematical Institute, University of Oxford, Oxford OX2 6GG, United Kingdom 